\title{
Workshop on
}

Micro-Mechanics

Measurement Technologies

for Fiber-Polymer Interfaces

Prepared by:

Walter G. McDonough

Gale A. Holmes

Donald L. Hunston

Richard S. Parnas

U.S. DEPARTMENT OF COMMERCE Technology Administration

National Institute of Standards and Technology

Gaithersburg, MD 20899-0001

May 28-30, 1997

QC

100

.456

N0. 6102 



\title{
Workshop on Micro-Mechanics \\ Measurement Technologies for Fiber-Polymer Interfaces
}

\author{
Prepared by: \\ Walter G. McDonough \\ Gale A. Holmes \\ Donald L. Hunston \\ Richard S. Parnas
}

U.S. DEPARTMENT OF COMMERCE Technology Administration National Institute of Standards and Technology

Gaithersburg, MD 20899-0001

May 28-30, 1997

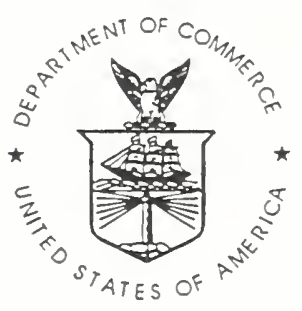

U.S. DEPARTMENT OF COMMERCE William M. Daley, Secretary

TECHNOLOGY ADMINISTRATION

Gary Bachula, Acting Under Secretary for Technology

NATIONAL INSTITUTE OF STANDARDS

AND TECHNOLOGY

Robert $E$. Hebner, Acting Director 



\section{Executive Summary}

The Polymers Division hosted the Workshop on Micromechanics Measurement Technologies for Fiber-Polymer Interfaces, jointly sponsored by NIST and the Textile Research Institute of Princeton, NJ, on May 28-30, 1997. Fifty researchers from industry, academia, and government laboratories attended the workshop to define the current status of measurement technology, get a hands-on demonstration of the latest technology, and identify the critical needs and opportunities for future research. The importance of the fiber-polymer interface for composite performance has inspired considerable research during the last decade on test method development to assess interfacial micromechanical properties. Although a number of tests have been developed, they all require many assumptions, and comparisons of test results from different laboratories have yielded poor agreement.

The most important need identified by the 15 attendees from industry was development of relationships between micro-mechanical testing and the performance of full-scale composites. This need was mentioned in several of the presentations and discussed in all of the discussion groups. Composite manufacturers would like to use databases of micro-mechanical test data on standard composite systems to design a broad array of composite products, and to minimize the expensive full scale testing currently required. However, to develop such databases, test methods must be reliable and standardized. Discussions of the prior inter-laboratory comparison of test methods revealed that a contributing factor to variability in results came from differences in testing procedures and sample preparation methods. Thus, standardization was identified as a key need. This led to a suggestion that micro-Raman spectroscopy be used as a quality control tool during the upcoming VAMAS round-robin testing program, which seeks to standardize sample preparation and testing protocols.

A questionnaire was given to all of the attendees and the following is the compilation of the results. Durability, mechanical properties, and cost were the overriding concerns of participants. However, it is puzzling that processing speed was not ranked higher since processing is a large fraction of the cost. This may be explained by the heavy concentration of aerospace applications where cost is important but speed is not. For implementing composites in commercial applications, durability and the establishment of acceptance criteria were most important, with manufacturing optimization a close second. For improving the usefulness of interfacial test methods, correlation of microtests with full composite behavior was most important, followed by test standardization. Among the participants, the single fiber fragmentation test is the most widely used test. Finally, the participants felt that none of the current tests are considered very useful although the indentation, single fiber fragmentation test, and short beam shear are marginal.

The workshop allowed theoreticians and experimentalists to get together to discuss the current state of micro-mechanics measurement technologies for fiber-polymer interfaces. Much was gained by allowing each side to learn about the challenges everyone faces in making these techniques more useful and reliable. For example, many comments were made about how the actual test data is generally reduced using simplistic models. New research on a more realistic analysis method relating the influence of matrix properties to the single fiber fragmentation test was shown during the workshop. This research showed conclusively that the DGEBA/m-PDA epoxy resin material exhibits nonlinear viscoelastic behavior during the fiber fragmentation process, hence, violating the linear elastic matrix assumption typically made in the development of shear lag models for determining 
interfacial shear strength. In addition, strain in the region between fiber breaks was shown to be approximately $25 \%$ to $30 \%$, while the global strain in the matrix is only $5 \%$. Results were also shown that indicate a significant perturbation of the stress field around each fiber break. These results were viewed by visiting researchers as significant and warranting further research effort. It was also noted that these results supported the need for new models and analysis methods to accurately determine the interfacial shear strength.

An important conclusion agreed on by both industry and academic researchers is that progress is occurring in both data analysis and measurement technology. Some test technologies are useful now for industry, some may never be, and there should be broad applicability to industry within ten years if similar progress continues. Finally, the meeting ended on the note that there should be a follow-up meeting in two to three years. 


\section{TABLE OF CONTENTS}

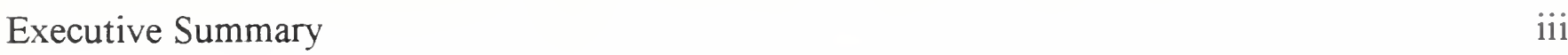

Summary of the Workshop 1

Reports from the Discussion Groups 5

Fiber Stress Analysis Techniques - L. Schadler - Rensselaer Polytechnic Institute 7

Test Analysis - J. Nairn - University of Utah,

P. Herrera-Franco - Centro de Investigacion Cientificas de Yucatan 15

New Methods - A. DiBenedetto - University of Connecticut, E. Pohl - Witco 19

Needs of Industry - T. Donnellan - Northrop-Grumman,

A. Netravali - Cornell University

$\begin{array}{ll}\text { Results from Questionnaire } & 25\end{array}$

$\begin{array}{lr}\text { Participants List } & 29\end{array}$

$\begin{array}{ll}\text { Appendix } & 35\end{array}$

Overview of Interface Testing

L. Drzal - Michigan State University $\quad 37$

The Microbond Shear Strength Pullout Test

B. Miller - Textile Research Institute/Princeton $\quad 67$

Statistical Analysis of Data from the Single Filament Composite

Test and Related Micromechanical Issues

L. Phoenix - Cornell University $\quad 85$

Comparison of the Interfacial Toughness Measured by the Single Fiber Pull-Out The Indentation, and the Fragmentation Tests

A. Hampe - Bundesanstalt fur Material Forshung und Prufung

Performance Property Relationships

D. Hunston - National Institute of Standards and Technology

Raman Techniques for the Analysis of Fiber and Composite Micromechanics

R. Young - University of Manchester and UMIST 



\section{Summary of the Workshop}

The Polymers Division hosted the Workshop on Micromechanics Measurement Technologies for Fiber-Polymer Interfaces, jointly sponsored by NIST and the Textile Research Institute of Princeton, NJ, on May 28-30, 1997. Fifty researchers from industry, academia, and government laboratories attended the workshop to define the current status of measurement technology, get hands-on experience with the latest technology, and identify the critical needs and opportunities for future research. The importance of the fiber-polymer interface for composite performance has inspired considerable research during the last decade on test method development to assess interfacial micromechanical properties. Although a number of tests have been developed, they all require many assumptions, and comparisons of test results from different laboratories have yielded poor agreement.

The most important need identified by the 15 attendees from industry was development of relationships between micro-mechanical testing and the performance of full-scale composites. Composite manufacturers would like to use databases of micro-mechanical test data on standard composite systems to design a broad array of composite products, and to minimize the expensive full scale testing currently required. However, to develop such databases, test methods must be reliable and standardized. Discussions of the prior inter-laboratory comparisons of test methods revealed that a contributing factor to variability in results came from differences in testing procedures and sample preparation method. Thus, standardization was identified as a key need. This led to a suggestion that micro-Raman spectroscopy be used as a quality control tool during the upcoming VAMAS, roundrobin testing program, which seeks to standardize sample preparation and testing protocols. An important conclusion agreed on by both industry and academic researchers is that progress is occurring in both data analysis and measurement technology, some technologies are useful now, and there should be broad applicability to industry within ten years if similar progress continues.

The meeting had six formal presentations, four discussion groups, a questionnaire, two poster sessions, a panel discussion on sample preparation techniques, and a tour of the micromechanical testing facility at NIST. The goal of the discussion leaders was not so much to discuss their research as to raise issues to be discussed in the subsequent discussion groups. Prof. Lawrence Drzal from Michigan State University served as the keynote speaker and presented an overview of interface testing. The other five speakers focussed on more specific topics. Dr. Bernard Miller, Textile Research Institute, presented work on the microbond shear strength pullout test. Prof. Robert Young, University of Manchester and UMIST, gave a presentation on Raman techniques for the analysis of fiber and composite mechanics. Prof. Leigh Phoenix, Cornell University, gave a presentation on the application of a new statistical theory for estimating interfacial shear strength and Weibull parameters for fiber strength for the single fiber composite test. Dr. Donald Hunston, NIST, talked on performance-property relationships, how the major objective for micro-mechanical tests is to provide information on composite behavior, and that the critical question is the relationship between micro-mechanical test results and the composite performance properties. Dr. Andreas Hampe, Bundesanstalt fur Material Forshung und Prufung (BAM), gave a presentation in which he compared the interfacial toughness as measured by the single fiber pull-out, the indentation, and the fragmentation tests. Their presentations can be found in the Appendix. The highlights of their presentations are discussed below. 
Prof. Lawrence Drzal, Michigan State University, served as the keynote speaker and presented an overview of interface testing. The goal of Prof. Drzal's presentation, and indeed for all of the presenters, was not so much to discuss their research as to raise issues to be considered in the subsequent discussion groups. The three questions posed in his presentation were first, is it necessary to measure fiber-polymer interface properties, second, how and why are fiber-polymer interface properties measured now, and third, what are the critical fiber-polymer interface properties to be measured? For the first issue, Larry felt that we do not need to measure fiber-polymer interface properties at the structural laminate level, but that we do need to measure the interface properties at the lamina level, i.e., how do fiber-matrix interface properties contribute to lamina properties, and how can we control and optimize fiber-polymer interface properties during fabrication. For the second issue, he identified composite lamina tests and microtests currently used to measure interface properties. He proceeded to go into some detail on both types of testing. For issue three, he listed the following characteristics that need to be measured: interfacial shear strength, transverse tensile strength, Mode I interfacial fracture toughness, Mode II interfacial fracture toughness, interphase modulus, interphase Tg and interphase failure mode.

Dr. Bernard Miller, Textile Research Institute, presented work on the microbond shear strength pullout test. He described the test methodology and pointed out how researchers can estimate the interfacial shear strength from the fiber diameter and the embedded length. However, he did acknowledge several sources of error that can lead to high standard deviations in test results. One source of error is droplets closer together along a fiber yielding strength values closer to each other than droplets tested further away from each other. This variation is due to different fiber surface conditions along the length of the fiber. Another source of error is if the volume of resin in the drop is reduced, then the contact angle between the drop and the fiber will become smaller. He then showed examples of the use and application of this test method.

Prof Robert Young, University of Manchester and UMIST, gave a presentation on Raman techniques for the analysis of fiber and composite mechanics. He explained how one makes measurements using Raman spectroscopy and how he applied this technique to examine poly-p-phenylene terephthalamide fibers encased in polymethylmethacrylate. He identified a universal calibration peak for aromatic polymer fibers and followed it as it changed values when the sample was placed in tension and compression. He showed similar results for high-modulus carbon fibers. Subsequently, he showed how the Raman technique could be applied to pull-out specimens, microbond specimens, single-fiber fragmentation specimens, and full composites. Although there is much potential with this technique, it cannot be used on glass fibers, opaque matrices, low or medium modulus carbon fibers, or on internal fibers in full composites.

Prof. Leigh Phoenix, Cornell University, gave a presentation on the application of a new statistical theory for estimating interfacial shear strength and Weibull parameters for fiber strength for the single fiber composite test. He used his quadratic influence superposition (QIS) technique to calculate stress and strain profiles in the fibers and matrix, the extent of the yield and debond zones, and the energy release rate in propagating the damage under increasing applied load. Insight was provided into the inverse relationship between the extent of debonding and the fiber spacing, variations in the fiber stress profiles as a result of coexisting elastic, yield, and debond zones, and finally, the interfacial parameters which give rise to stable longitudinal damage growth. A key result is that the total energy 
release rate varies strongly with the fiber spacing, suggesting that this quantity, as measured from a single-fiber composite test, is not an intrinsic quantity governing damage growth around a fiber break in a fibrous composite.

Dr. Donald Hunston, NIST, talked on performance-property relationships, how the major objective for micro-mechanical tests is to provide information on composite behavior, and that the critical question is the relationship between micro-mechanical test results and the composite performance properties. He discussed uncertainties in the different micro-tests such as how the tests are being performed and what does each test measure. He talked about the different micro-mechanical tests and how they yield different strength values but follow similar trends. Subsequently, he compared these results with those measured on bulk composites. Among the problems with the micro-tests, he identified two main ones. The first is the processing challenge, that is, micro-samples need to be made in the same way that the full composite is made. The other is that we need to realize that each micro-test has limitations, so multiple tests are desirable. He then proceeded to discuss the application of single fiber tests to durability studies being conducted at NIST. In particular, he talked about the effects of silane coupling agents on durability. This study showed that the glass-epoxy interface is attacked by moisture, that chemical bonding improves durability, and that the results on fibers showed the same trend as results from tests on flat plates. He finished his presentation by discussing the results from a round robin study performed in 1990 where the same test was conducted by several labs around the world. There was a lot of disagreement in the data among the laboratories in that study that was attributed to lack of standardization in the test procedure and sample preparation. A new round robin is being conducted under the auspices of the Polymer Composites Working Group of VAMAS. The objectives will be to develop a recommended test procedure, to conduct tests with a single batch of samples following the recommended test procedure, and to provide a forum for identifying critical research issues.

Dr. Andreas Hampe, BAM, gave a presentation in which he compared the interfacial toughness as measured by the single fiber pull-out, the indentation, and the fragmentation tests. The first part of his presentation focussed on the case of pure ductile failure of the interphase. When this type of failure occurs, the Kelly-Tyson equation can be used to determine a value for the interfacial shear strength for either the fragmentation test or the pull-out test. The second part of his presentation focussed on brittle failure. With the fragmentation test, the stress distributions become more complicated. Due to the brittle behavior of the matrix, he found a debonded zone with frictional stresses and a bonded region with a shear stress maximum at the crack tip. He concluded that, since we do not get any information on these frictional stresses, we do not get enough information with the fragmentation test to determine a shear strength. With the single fiber pull-out test, from the pull-out force and the embedded length, he could calculate the frictional shear stress. By subtracting the friction force from the measured force, he was able to get the force at the crack tip and concluded that the pull-out method is a good choice for interface studies. Not surprisingly, the attendees expressed a wide range of views on the merits of the various test methods in the discussions that followed the presentations. Each test had it's supporters and detractors with strong reasons for their opinions. Several people reiterated Dr. Hampe's point that it is important to use the latest data analysis procedures to help address some of the deficiencies in the various test methods. The overall conclusion was that each test has strengths and weaknesses that need to be understood so that the best choice can be made for the particular materials and conditions to be studied. 
Following the individual presentations, four discussion groups were held over the course of two afternoons, with the results from the discussion groups being presented on the last day of the meeting by the discussion leaders. The main conclusion of the Needs of Industry discussion group, led by Dr. Thomas Donnellan from Northrop-Grumman and Prof. Anil Netravali from Cornell University, was that the industry would appreciate a test that is simple, standardized, reliable, inexpensive, rapid, and that yields critical information. The main conclusion of the Fiber Stress Analysis Techniques discussion group, led by Prof. Linda Schadler from Rensellaer Polytechnic Institute, was that fiber stress analysis techniques offer a unique method for gathering micromechanical information. These may be the only methods by which we can understand the initiation of damage and the role of the interface in damage initiation sufficiently quantitative to provide input into predictive failure models. In order to take full advantage of the capabilities of these techniques, teams of people with experts in both modeling and experimental techniques are required. Finally, possible industrial uses for fiber stress analysis techniques exist, especially as the instrumentation improves and the capital costs are reduced. The main conclusion of the Test Analysis discussion group, led by Prof. John Nairn from University of Utah and Dr. Pedro Herrera-Franco from the Center for Scientific Research in Mexico was that, although it is widely recognized that stress state differences exist among the various micromechanical testing methods, the reduction of experimental data traditionally follows simplistic methods. Many differences exist in the mechanical behavior of the materials tested, so more realistic theoretical analyses were considered. Finally, the New Methods discussion group, led by Prof. Anthony DiBenedetto from the University of Connecticut and Dr. Eric Pohl from Witco Corporation, concluded that any new method needs to provide straightforward data analysis and interpretations, be cost effective, be able to analyze realistic models, be able to translate micromechanical results to composite properties, and be reproducible. More detailed descriptions of the conclusions of the discussion groups can be found in the main section of the text.

In addition to the formal presentation and discussion groups, the attendees also filled out a questionnaire which gave some interesting insights. Durability, mechanical properties and cost were the overriding concerns of the participants. When trying to use composites in commercial applications, durability and the establishment of acceptance criteria were the most important issues, with manufacturing optimization a close second. Correlation of the microtests with full composite behavior was the most important factor, followed by test standardization for improving the usefulness of the interfacial test methods.

During two of the three days, a poster session was held for the attendees to share their research results. In addition to the poster sessions and concurrent with the discussion groups, there were tours of some of the different single-fiber test methods. Finally, a panel discussion was held on sample fabrication issues. The main conclusion of the panel discussion was that more standardization needs to be done not only in how the different micromechanical tests are done, but also in how the test samples are made.

In conclusion, the workshop allowed theoreticians and experimentalists to get together to discuss the current state of micro-mechanics measurement technologies for fiber-polymer interfaces. Much was gained by allowing each side to see how the other half approaches their work and learn about the challenges everyone faces in making these techniques more useful and reliable. The meeting concluded on the note that there should be a follow-up meeting in two-three years. 


\section{REPORTS FROM THE DISCUSSION GROUPS}



Fiber Stress Analysis Techniques Discussion Group Report

L. S. Schadler, Rensselaer Polytechnic Institute

INTRODUCTION

This document presents the results of a discussion on "Fiber Stress Analysis Techniques" held during a National Institute of Standards and Technology workshop on

"Micromechanics Measurement Technologies for Fiber / Polymer Interfaces" held May 28-30, 1997. Thus, while the author put the words to paper, the opinions and information presented are the result of several hours of discussion with the scientists and engineers listed below. Therefore the information and opinions cannot be assigned to individual participants and there was healthy disagreement around our "round table."

For more information on the techniques there is a list of review articles and a few specialized articles at the end of this report.

The report begins with a definition of fiber stress analysis techniques and a bit of background on Raman and Fluorescence microscopy. This is followed by a discussion of two questions: 1) Can micromechanical experimental information be used to make macroscopic predictions?, and 2) How can these techniques be used in the industry and to advance our understanding of micromechanics?

\section{DEFINITION}

Fiber stress analysis techniques were defined by the group as those techniques which allow measurement of the stresses or strains in an embedded fiber or the matrix surrounding the fiber with micron spatial resolution. The discussion centered primarily on Raman and Fluorescent microprobes (or micro Raman and Fluorescence spectroscopy). The spatial resolution of photoelasticity is high, but the strain is averaged through the sample, and thus was not considered in our discussion. Time resolved spectroscopy was noted as an emerging technique and it was acknowledged that there are many surface techniques that can infer information below the surface.

\section{CAPABILITY AND LIMITATIONS OF RAMAN AND FLUORESCENCE MICROSCOPY}

The Raman phenomenon is an inelastic scattering of light. If monochromatic light with frequency $v_{0}$ is scattered by molecules or a crystal, the majority of the scattered light will have the same frequency as the incident light (elastic or Rayleigh scattering). A small fraction of the scattered light, however, will experience a change in its frequency and will have a frequency $v_{0} \pm \Delta v$ (inelastic or Raman scattering). The change in the frequency of the scattered light $(\Delta v)$ is equal to the frequency of the natural vibrational modes in the scattering material. In crystalline materials and highly oriented polymers, changes in the crystal symmetry due to an applied strain will often cause changes in the frequency or wave number at which Raman scattering achieves peak intensity (i.e. Raman peak position of the material). The Raman peak position of the Stokes lines for affected tend to shift linearly to lower wave-numbers under tensile strains (higher frequencies) and to higher wave-numbers under compressive strains (lower frequencies). Figure 1 shows a Raman peak for graphite fiber shifting under tensile strain. 


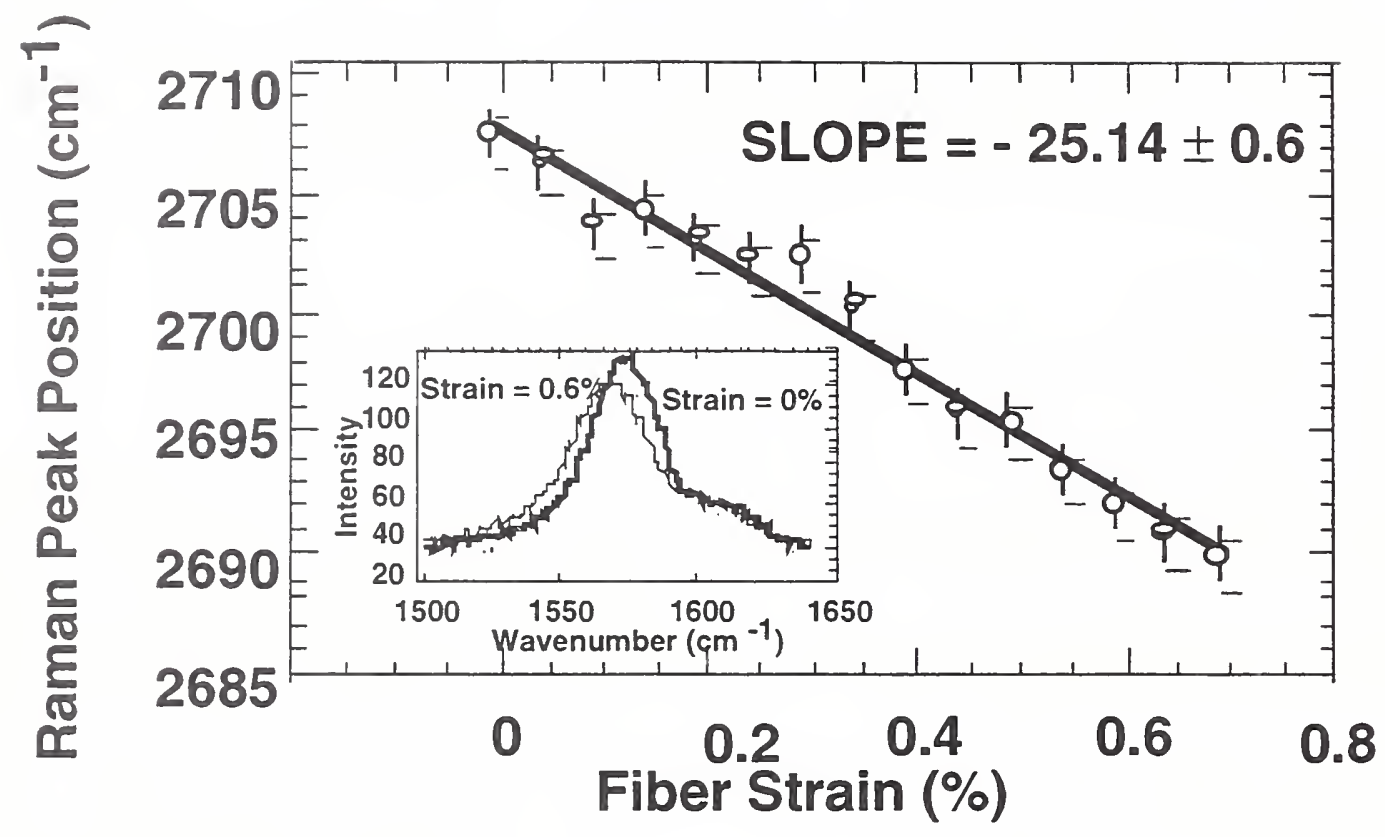

Figure 1. The linear dependence of Raman peak position on applied strain for HM graphite fibers.

Using the calibration curve of a fiber in air shown in Figure 1, the axial strain distribution along a fiber embedded in a transparent matrix can be determined by taking Raman spectra of the fiber at different points along its length. The fiber strain, $\varepsilon_{\mathrm{i}}$, at any point i can be calculated according to:

$\varepsilon_{\mathrm{i}}=\left(\Delta v_{\mathrm{i}}-\Delta v_{0}\right) / \mathrm{SLOPE},(1)$

where $\Delta v_{\mathrm{i}}$ and $\Delta v_{0}$ are the peak positions at point $\mathrm{i}$ and at zero strain respectively. Reference 1 gives a review of micro Raman microscopy in fibers and polymer composites.

Fluorescence microscopy can be used on some materials with a fluorescence peak, for example, alumina fibers [2]. The fluorescence $\mathrm{R}$ lines in alumina have been found to shift with applied strain with a slope on the order of $10 \mathrm{~cm}^{-1}$ per percent strain. The strain in the fiber can then be found using equation 1 where the SLOPE is obtained from a calibration curve similar to that in Figure 1.

With the ability to measure strain in fibers, several other micromechanical measurements can be made. For example, residual stresses and strains [3], strains around a defect such as a hole or a crack [4], early damage such as the strain concentration factor around an individual fiber break [5] (Figure 2). These strain measurements can be made directly using equation 1 and with remote testing systems now available the possibilities for durability, creep, fatigue, and other loading geometries is almost endless.

In addition to strain profiles obtained using fiber stress analysis techniques, the interfacial shear stress (ISS) profiles can be calculated without assuming anything about the interface [6]. This is a strength compared to most micromechanical interface tests which must assume a constant ISS. The ISS distribution $\tau_{\mathrm{i}}$ along the fiber length $\mathrm{x}$ can be calculated using a simple force balance which yields: 
$\tau_{\mathrm{i}}=(\mathrm{E} \mathrm{D} / 4)\left(\mathrm{d} \varepsilon_{\mathrm{i}} / \mathrm{dx}\right)(2)$

$\tau_{\mathrm{i}}$ is the shear stress at the interface at any point $\mathrm{i}$ while $\mathrm{E}$ and $\mathrm{D}$ are the fiber Young's modulus and fiber diameter, respectively. Figure 3 shows a typical strain profile on a graphite fiber fragment and the resulting interfacial shear stress. From the ISS profile, the stress transfer behavior can be understood. For example, a constant ISS with a high value close the shear yield stress of the matrix indicates that the interface is well bonded and that matrix plastic deformation limits the load transfer. A constant interfacial shear stress with a low value (e.g. $10 \mathrm{MPa}$ ) indicates a frictional stress transfer mechanism due to debonding or weak interfacial shear strength.
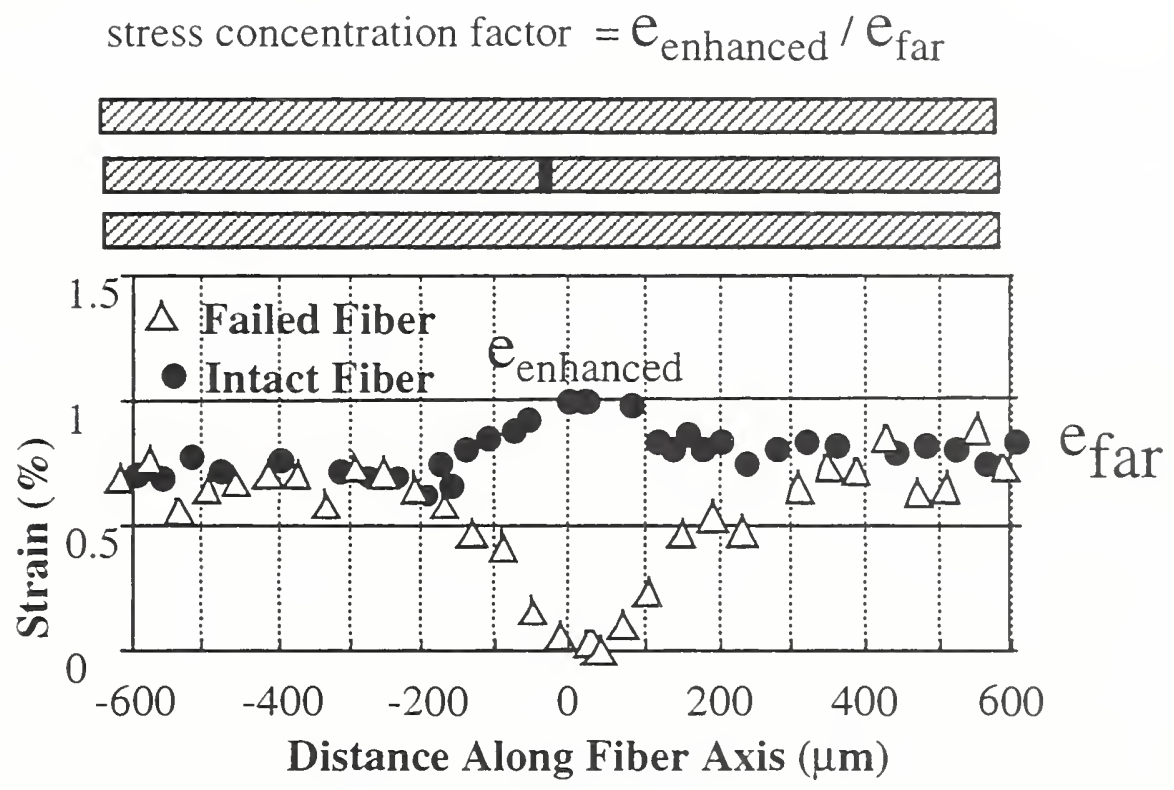

Figure 2. The strain profile in a failed fiber and the neighboring intact fiber showing the enhanced strain region as measured using micro Raman spectroscopy (MRS).

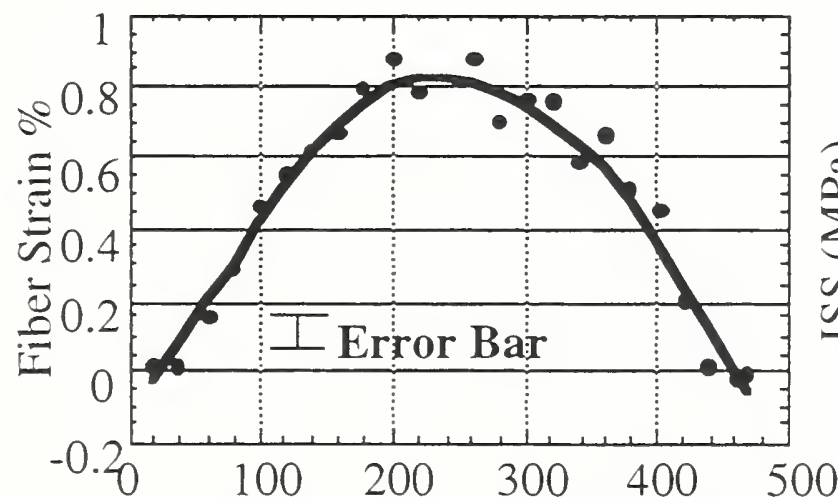

Distance from Fiber End $(\mu \mathrm{m})$

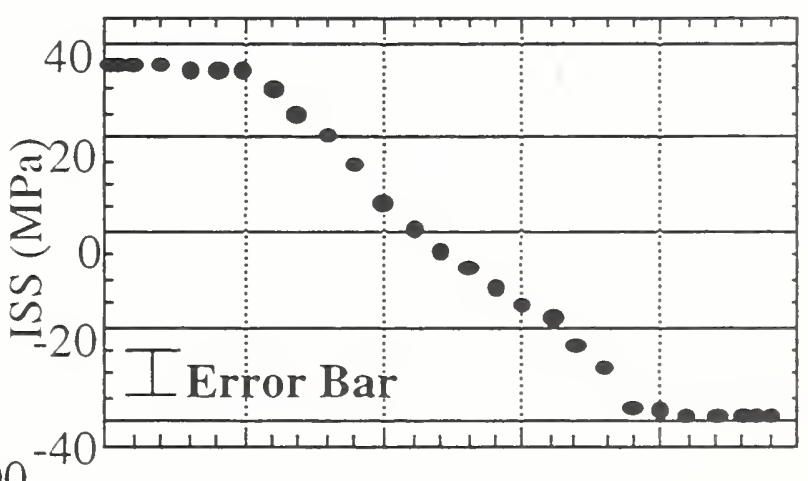

Distance from Fiber End $(\mu \mathrm{m})$

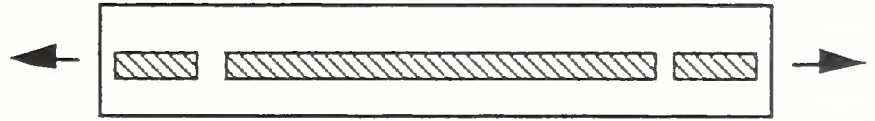

Figure 3. Strain and ISS profiles as measured using the Micro-Raman Spectroscopy technique. 
There are several limitations of these techniques. They are listed below:

1) The matrix must be transparent. Otherwise these techniques only give information about strains on the sample surface.

2) Not all fibers show a shift in the Raman or Fluorescence peak position with applied strain. Glass is the most noted example of this.

3) Only the first few layers of fibers can be studied. If one fiber lies beneath another, the laser beam cannot reach the underlying fiber.

4) Only axial strains are measured in the fibers. As volume fraction increases, the strain state is complicated and the axial strain may not give enough information to develop predictive models. In general the technique can be used to measure complex states in materials, but this has not been extended to high performance fibers and may be impossible due to their structure.

5) The measurements are time consuming.

CAN MICRO INFORMATION BE USED TO MAKE MACROSCOPIC PREDICTIONS?

The overwhelming answer to this question was - maybe.

Twenty years ago the micromechanical modeling was well ahead of the experimental information available. The models, however, assumed elasticity and perfect bonding at the interface. Some properties such as modulus can be predicted from these early models. On the other hand models predicting strength, creep rupture, fatigue, fracture require more complex modeling. Using micromechanical test techniques combined with macroscopic testing, it was learned that the interface plays a crucial role in determining macroscopic properties and cannot be ignored in the models. In addition, using fiber stress analysis techniques, it is now possible to study the constitutive behavior surrounding a fiber break or near a defect. With information about interfacial behavior and the constitutive behavior around a break or defect, models that predict damage and failure should be possible. Closed form solutions are not appropriate, but finite element modeling can give insight into micromechanical behavior and help in the interpretation of micromechanical tests. To predict failure, however, computational techniques must be used and developed that can handle a large number of fibers and include the statistics of fiber failure. If failure can be modeled in a laminate of general loading geometry, laminate theory is well enough developed that, ideally, failure of real parts could be predicted.

On the other hand, the geometry of the fibers must be well understood for the models to work. Therefore, prediction of failure in injection molded short fiber composites might be beyond our capabilities at the moment. The complexity of anisotropy in both the matrix and fiber orientations and changes in anisotropy through the thickness of the sample give an added complexity to the problem. We are much further from solving this problem.

Several points were made to the experimentalists in the field.

1) The micromechanical behavior is a function of volume fraction. For example, the residual stresses due to processing change with volume fraction; as the volume fraction of fibers increases, the effect of matrix shrinkage decreases. Compression failure is particularly sensitive to the volume fraction; kink bands do not form in single or multi fiber composites, but do form in bulk composites. In addition the energy absorbing mechanisms 
change as a function of volume fraction.

2) When comparing experiment to theory, make sure that the geometry of the model is the same as the experiment.

Several points were made to everyone in the field.

1) The models must include interface behavior.

2) The experimentalists and theoreticians need to form more teams. The process of developing predictive models needs to be more iterative with experiments. The same geometries need to be tested to compare the predictions of the models and to develop parameters for the models. Once bulk predictions are made, experiments need to be run on bulk samples to verify the models.

\section{WHERE CAN THESE TECHNIQUES BE USED TO ADVANCE THE FIELD?}

Fiber stress analysis techniques are still under development and as the equipment becomes more inexpensive and easy to use and align, there are applications in industry. In addition, the limits of the technique to give micromechanical information need to be pushed. This was a very fruitful part of our discussion because both industrial and academic insights were obtained.

One obvious use of fiber stress analysis techniques in the industry is to measure residual stresses in both prepregs and final parts. In addition, other micromechanical test methods are influenced by residual stress and this can be accounted for by using them in conjunction with a fiber stress analysis technique. In addition, by placing an optical fiber into a mold and gathering the Raman signal, the development of residual stresses might be studied.

A second application in industry is for quality control on a fiber processing line. For example, the Raman peak shape and the shift in Raman peak position with applied strain is sensitive to carbon / graphite fiber structure [7]. Therefore, the Raman signal could be used for quality control in a carbon / graphite fiber processing plant. It might also be possible to use the Raman to detect defects in PAN fibers before carbonizing.

The rest of the discussion focused on pushing the limits of the techniques for micromechanical measurements. One area that has room for growth is using the technique in more types of materials. For example, the stress in particulates can be measured with these two techniques. In order to understand the reinforcing mechanism in particulate composites, it would be helpful to understand how much strain the particulate is carrying. Secondly, are there other optical phenomenon in low modulus carbon and glass fibers that would shift with applied strain? It would be beneficial to be able to study these systems because the interface, especially in glass fiber composites, is very different from highly oriented polymer or graphite fibers studied to date.

Finally, recent work done in Robert Young's group [8] got the group quite interested because it opened up the possibility of studying the stress and strain in glass fiber composites. Thongpin et al. have taken diacetylene and made it into diols that can be processed with urethanes to make coatings that show a Raman shift with applied strain. The Raman signal is a resonance signal with a HeNe laser and thus has high intensity. It was thought that inclusion of the diol into glass fiber sizings would be possible. Because of the strong signal only a small percent of diacetylene would be required. This would provide a method for studying the strain in the interface region in glass fiber composites. In addition, it was speculated that the signal along a fiber could be measured using 
evanescent waves. Thus the average strain in glass fibers could be measured during processing or in use!

\section{FINAL THOUGHTS}

One of the major limitations of these techniques is that strain is obtained point by point. If a line focusing technique could be developed, the second dimension of the CCD could be used and strain along a section of fiber could be determined from one reading. This would greatly improve the efficiency of the technique.

Many people are entering this once small subfield. In order to ensure high quality information, a standard method of testing was discussed. For example, should a laser power range be standardized. High laser power can cause peak shifts because of a change in temperature. Should a standard signal to noise ratio be established? Peak fitting varies from group to group and so do the methods of determining the slope in the strain profiles. This can alter the results and / or give false data. Should this be standardized? These questions were not answered by the group.

\section{CONCLUSION}

Fiber stress analysis techniques offer a unique method for gathering micromechanical information. These may be the only methods by which we can understand the initiation of damage and the role of the interface in damage initiation quantitatively enough to provide input into predictive failure models.

In order to take full advantage of the capabilities of these techniques teams of people with experts in both modeling and experimental techniques are required.

Possible industrial uses for fiber stress analysis techniques exist, especially as the instrumentation improves and the capital costs are reduced.

\section{REFERENCES}

1. L.S. Schadler and C. Galiotis, "Fundamentals and Applications of Micro Raman Spectroscopy to Strain Measurements in Fibre Reinforced Composites," International Journal of Materials Reviews, vol. 40, no.3, pp.116-134 (1995).

2. R.B. Yallee, M.C. Andrews, and R.J. Young, "Fragmentation in Alumina Fibre Reinforced Epoxy Model Composites Monitored Using Fluorescence Spectroscopy," Journal of Materials Science. vol. 31, no. 13, pp. 3349-3359 (1996).

3. X. Yang and R.J. Young, "Model Ceramic Fibre-Reinforced Glass Composites: Residual Thermal Stresses," Composites, vol. 25, no. 7, pp. 488-493 (1994).

4. J.A. Bennett and R.J. Young, "Micromechanical Aspects of Fibre/Crack Interactions in an Aramid/Epoxy Composite," Composites Science and Technology, in press (1997).

5. L.S. Schadler, M.S. Amer, and B. Iskandarani, "Experimental Measurement of Fiber / Fiber Interaction Using Micro Raman Spectroscopy," Mechanics of Materials, vol 23, pp. 205-216 (1996).

6. C. Galiotis, "Interfacial Studies on Model Composites Using Laser Raman

Spectroscopy," Composites Science and Technology, vol. 42, pp. 125-150 (1991). 
7. N. Melanitis, C. Galiotis, P.L. Tetlow, and C.K.L. Davies, "Characterization of Carbon Fibre Surfaces and Examination of Carbon Fiber / Epoxy Interfaces with Laser Raman Spectroscopy," Journal of Composite Materials, vol. 26, pp. 459-466 (1992).

8. C. Thongpin, R.J. Young, J.L. Stanford and P.A. Lovell, "Development of Diacetylene-Urethane Copolymers for Strain Measurement Applications in Glass Fibre Composites" ECCM-7, Seventh European Conference on Composite Materials 14-16 May 1996, vol. 2, p.41 Woodhead Publishers Ltd., Cambridge (1996). 



\section{Test Analysis Discussion Group Report \\ Dr. John Nairn, University of Utah \\ Dr. Pedro Herrera-Franco, Centro de Investigacion Cientificas de Yucatan, Mexico}

This discussion session discussed the various micromechanical testing methods including the microbond test, the single fiber micro-indentation test, the fragmentation test, and the single-fiber pull out test. The participants made comments about the strengths and weaknesses of each test. This report focuses on the weaknesses that were pointed out and those points which were common to all techniques which were considered to be relevant and that are possible topics of further research.

The first point which was mentioned was the need for better schemes for reduction of experimental data. Some of the considerations to be made in analytical methods include the differences in the state of stress which is imposed on the interface/interphase. Although it has been widely recognized that stress state differences exist, the reduction of experimental data traditionally follows simplistic methods. In the case of the microbond and pull-out techniques, only an average value of interfacial shear strength is calculated. This calculation is simply the value of the force for debonding the droplet or pulling out the fiber divided by the embedded surface area of the fiber. In the case of the single fiber fragmentation. the data reduction scheme uses an oversimplified equation developed by Kelly and Tyson together with a statistical analysis incorporated to recognize the random nature of the fiber fragmentation process. For the micro-indentation test, the experimental data are reduced using an

algorithm developed from experimental data collected from several fiber/matrix combinations and coupled to a very simplified finite element analysis.

Another topic discussed was the need to establish suitable failure criteria in the analysis of the different techniques. These criteria should be included in any analysis developed for reducing the experimental data. Some results have shown that depending on the degree of fiber/matrix adhesion, a crack can grow either along the interphase or into the matrix. For strong fiber-matrix adhesion, matrix yielding is usually observed. It was mentioned that, in view of the differences in failure modes, the mechanical behavior of the matrix needs to be addressed. This was considered to be an important point since many theoretical analyses consider linear behavior, but at larger deformations such mechanical behavior could become non-linear. Another point was that the analysis of the single fiber fragmentation test should account for the large deformations that have to be induced in the matrix in order for the fiber fragmentation process to reach the saturation point. It was further mentioned that in real composites, common designs would not permit such large deformations to occur. Perhaps the results at the saturation damage state are not representative of the role of the interface/interphase in real laminates. The viscoelastic behavior exhibited by most polymers was also mentioned as an important parameter for test analysis. Also, differences in behavior between thermoplastic and thermosetting polymers were considered to be important parameters of analysis.

In view of such differences in mechanical behavior, especially in the failure mode, possible approaches for more realistic theoretical analyses were considered. Many models have used strength methods, but there is a growing trend to use fracture mechanics or energy methods. One important point of agreement among the analysts was the lack of experimental data about interphasial mechanical properties. Most theoretical analyses, either strength or energy based, always have to assume some interphase mechanical properties, and thus, those properties are needed for input to the models. 
A particular concern about interface/interphase properties is that we should distinguish between mechanical properties of the interphase and failure properties. Mechanical properties of the interphase means the ability of the interphase, whether damaged or undamaged, to transfer stress from the matrix to the fiber. This property is especially important when there is an interphase region of finite thickness. Such mechanical properties are distinct and independent from failure properties of the interface/interphase. Any micromechanical analysis of interface tests must account for both mechanical and failure properties of the interface to have a chance of being correct. It should be considered impossible to deduce both types of properties from a single interfacial failure test. In general, the common interfacial failure tests give information about failure, but because that failure is influenced by the mechanical properties of the interphase, it seems unlikely that such data can ever be accurately analyzed without combining the results with supplemental experiments on interphase properties. Two suggested techniques were Raman spectroscopy and photoelasticity which, when correctly analyzed, can give information about interphase mechanical properties without interference from failure properties.

A related complicating feature of data analysis is the role of interfacial friction. As with interphase mechanical properties, friction plays a role in interface failure, and it seems doubtful that accurate interfacial failure properties can ever be deduced without combining the interface tests with supplemental experiments that give the magnitude of the frictional stresses. The modeling of friction is also very complex, and most models simply model friction by a constant shear stress boundary condition on the crack faces or by an interface condition based on Coulomb's friction law. Neither of these processes correctly includes friction as a non-conservative, energy dissipation mechanism. They may, however, be adequate and are certainly better than ignoring friction when it might be significant.

Another topic of research was the need for establishing a standard experimental protocol for each of the techniques. It was considered that theoretical modeling could provide valuable information to conduct the experiments and to reduce the experimental data in a more realistic manner. As an example, the rate of loading at which the different tests need to be conducted was mentioned. This rate was considered as an important parameter since polymer behavior was dependent on time and temperature. A thorough analysis could provide guidelines for proper experimental procedures and for a better interpretation of interface/interphase failure. The extent of loading was another experimental parameter that needs further analysis. In the case of the single fiber fragmentation test, a question was posed as to whether a load needed to be applied until saturation of the fragmentation process was reached, or whether it was only necessary to analyze fiber fracture that occurred at low levels of applied stress. Perhaps the best choice for fragmentation tests is to always record the number of breaks as a function of strain; then both the low level and the saturation level data will be available for subsequent data reduction. In the case of the micro-indentation test, the depth of indentation was considered to be an important parameter for further analysis. In this case, the difficulty of real time observation and the lack of evidence for fiber-matrix debonding makes the analysis necessary.

One of the experimental shortcomings which was considered to be common to all the techniques was the difficulty to record crack initiation and growth. Crack observations are particularly important for many fracture mechanics or energy models. Also, some of the techniques suffered from a lack of 
reproducibility between different laboratories and in some cases within the same laboratory. Another point of interest was that, in a real composite, there are multiple fiber interactions while most of the micro-mechanical tests do not consider such effects.

The final point, and perhaps the ultimate goal or reason for all interface tests, is that we need to establish a relationship between the micro-mechanical behavior and the macro-mechanical behavior. Specifically, how can the lab tests give quantitative information about the role of the interface/interphase in influencing the properties of real composites? 



\section{Discussion Group on New Methods \\ A.T. DiBenedetto, University of Connecticut Eric Pohl, Witco}

The discussion group on New Methods was charged with the task of exploring new micromechanical test methods and procedures for providing quantitative information regarding the relation between interfacial properties and the mechanical behavior of fiber reinforced composites. The general questions that guided the discussions were:

(a) Have new concepts or methods been developed in the recent past that enable one to define accurately the axial stress carried by the reinforcing fibers and the toughness of the fiber/matrix interface, for example the shear stress transmission parameter, $\tau$, and the debonding energy $\mathrm{G}$; and can presently available or new micromechanical test methods provide the means for defining failure mechanisms in composite materials?

(b) Are presently available or new micromechanical tests useful tools for industrial product development; and can they be developed as standardized tests for characterizing the interfacial properties of composite materials?

It is easy to understand why there is concern over correlation of interfacial properties with composite properties, since many mechanical properties of fiber reinforced composites depend strongly on the efficiency of stress transfer between matrix and fiber. In continuous fiber composites, it affects shear strength, shear modulus, off-axis properties, compressive strength, flexural strength, impact strength, fatigue properties and environmental durability. In short fiber composites, on-axis strength and modulus are affected in addition to all of the above-mentioned properties.

Of the numerous micromechanical test methods reported in the literature, the embedded single fiber fragmentation test and one or two variations of fiber pull-out tests have been the most commonly employed. While there are no truly new micromechanical test procedures that have received wide-spread attention, there are several analytical and experimental techniques that provide the necessary analytical data to establish reliable values for "fundamental" interface properties. These developments are important since they allow for the possibility of establishing a standardized data base upon which correlation of interfacial properties with fiber reinforced material properties can be tested. The following advances that have appeared in the (more or less) recent literature were considered noteworthy.

(1) Laser Raman Spectroscopic Measurement of Strain in Fibers.

The technique is discussed in detail in other discussion groups in this workshop. The work first done by R. J. Young and then by C. Galiotis and L. Schadler enables the direct measurement of the axial stress profile in Raman spectra sensitive materials such as carbon fibers. This removes the necessity of a qualitative measure of fiber efficiency in terms of a fiber critical length, as defined by mathematical models such as the Kelly-Tyson and Cox models. Thus, when a Laser-Raman instrument is available, one can measure directly the load bearing capacity of a fiber in both a single fiber microcomposite and a fiber on the surface of a fiber reinforced composite material. The availability of portable spectrometers enables study of the effect of extended service on fiber 
efficiency and on the extent of composite damage at the end of a filament fragment.

(2). Analytical Definition of Axial Fiber Stress in Terms of "Imperfect Interfaces".

An important accompaniment to Laser-Raman experiments has been the introduction by $Z$. Hashin (in 1990) of the concept of imperfect interfaces in composite materials. In his seminal work, he defines mathematically a set of three damage parameters, $D_{j}$, that define the load bearing capacity of a fiber-matrix interface. In 1996, J. Nairn and colleagues developed analytical expressions for the axial stress profile along a fiber fragment in a single fiber composite in terms of a damage parameter, $D_{s}$, and an infinite series of Fourier-Bessel functions. Thus, using an experimentally measured axial stress profile, one can assign a specific value, or values, of a damage parameter to the load bearing capacity along the fiber-matrix interface. This combination of experimental and analytical information provides a fundamental basis for evaluating the results of a micromechanical test procedure.

Thus, for carbon fiber composites and other fibers active in Raman spectra, one can measure strain profiles directly using laser-Raman spectroscopy and compare the results with those simulated by analytical and finite element models. Both the modes of failure and stress transfer characteristics can be observed and verified in separate experiments. This can also be done "on-line" during composite testing to study the durability of the material and to establish the usefulness of micromechanical testing. For glass fibers and other non-active reinforcements, one must use alternative methods for direct measurements of strain and debonding conditions. Members of the discussion group proposed the combined use of photolithography, atomic force microscopy, and other optical techniques for measurement of the stress transfer characteristics of the fiber-matrix interface.

A new method of measuring directly the interfacial normal strength has been proposed by P. F. M. Meurs, P. J. G. Schreurs and T. Peijs (1997). Using the electron beam of a scanning electron microscope (SEM), one can create on the transversely cut surface of a composite material (or microcomposite) a set of markers (spots) around the fiber-matrix interface. One can then load the material in the field of the SEM and observe the change in the distance between markers as a function of load, until a point of separation occurs at the interface. A numerical simulation of the experiment is made using the markers as boundary conditions for a finite element analysis. The simulation is used to calculate the displacements of the markers as a function of the interphase modulus. When the computed displacements match the experimentally observed ones, both the elastic properties and the normal strength of the interphase are defined. The fiber efficiency, that is, the load bearing capacity of the fiber-matrix interface, is well defined by the advances described above. Future efforts will certainly lead to an answer to the question of whether micromechanical test results will correlate directly with fiber composite performance. Furthermore, the use of single fiber microcomposite testing enables observation of the modes of damage occurring at a fragment end and how the mode of fracture changes during the lifetime of the material.

The state-of-the-art of measurement and interpretation of the fracture energy, $\mathrm{G}$, occurring during fiber fragmentation and pull-out is less advanced. Analytical energy-based solutions have been proposed by numerous investigators, including Piggott, Nairn, Phoenix and Wagner. Finite element analyses have also been reported by numerous investigators, including DiBenedetto, Hampe, Marotske, Tripathy and Jones. While it appears that an energy-based interpretation of fracture processes in microcomposites is relevant to the fracture toughness of the interface and, therefore, is 
key to an understanding of the long-term durability of a composite, the utility of micromechanical measurement of $\mathrm{G}$ is still unknown. The absolute values of $\mathrm{G}$ presented in the literature for similar systems vary considerably. Some authors use an overall energy change per unit area of debond, $-\Delta \mathrm{U} / \Delta \mathrm{A}$, to characterize the interfacial debonding, and others use the fracture mechanical definition, $\mathrm{G}=-\partial \mathrm{U} / \partial \mathrm{A}$. Most reported analyses assume that the polymer matrices and interphases are linear elastic materials, while it is widely recognized that their elastoplastic and viscoelastic properties will affect calculated values of debonding energy. Other irreversible processes, such as friction and acoustic energy dissipation that accompany filament fracture and debonding, are also ignored. Both analytical and finite element analyses that consider these factors are now being presented at conferences and showing up in the refereed literature. However, there does not appear to be a consensus view on how to proceed and, therefore, no standard approach that will allow independent experimental verification of reported results.

It was suggested that a more fundamental fracture mechanics approach utilizing a planar reinforcement-matrix interface, such as the experimental techniques and analyses reported by Kramer, Brown, Kanninen and others for the study of interfacial debonding at polymer/polymer interfaces might provide guidance for future studies of fiber-matrix interfaces. For example, if surface treated reinforcing tapes or fibers could be sandwiched between two reinforcing plates, a standard fracture mechanics test could be utilized to study the effect of surface treatments on the resulting composite.

The second general question related to the usefulness of micromechanical tests is related to their value as tools for guiding the industrial development of commercial materials. From an industrial perspective, their principal uses are:

- to develop structure-property relationships on simplified model systems

- to organize empirical data in such a way as to guide decision making regarding step-changes or optimization of a particular composite material

- to help explain test results on composite products

- to be useful as a predictor of durability, corrosion resistance and other composite properties

In order to be accepted by the industrial community, the micromechanical tests must:

- provide for straightforward data analysis and interpretations

- be cost effective in terms of the time required to develop, carry out and analyze the required tests, and to train the laboratory personnel

- show a clear relationship between the micromechanical test results and composite properties

- be reproducible in a standardized procedure

With regard to the latter question of reproducibility, the recent round robin study reported by Pitkethly, et al., (1993) on the reproducibility of pullout, microbond, fragmentation and indentation microcomposite tests indicated that in general such tests were reproducible within the procedures of a specific laboratory, but were not reproducible in comparison of results from the different laboratories participating in the round robin study. It is felt that a round robin study should be repeated with very specific guidelines required with respect to sample preparation, analysis of fiber properties and other test conditions. It is recommended that the VAMAS project sponsored by NIST be used to reinvestigate this question. 
At this time, there is sharply divided opinion as to whether micromechanical testing meets all of the requirements specified above. Some industrial scientists have utilized these tests to guide their development work, while others suggest that they are not as useful as more traditional macromechanical tests, such as short beam shear tests and transverse tensile testing of real fiber reinforced materials. The principal reservations are concerned with the cost effectiveness of the tests and the lack of clear proof that there is a correlation between micromechanical test results and composite properties. There is a generally accepted feeling that if these questions can be resolved, a combination of micromechanical and macromechanical testing will be valuable in directing product development.

There is a strong consensus that the use of Laser Raman spectroscopy for the direct measure of axial fiber strain in Raman sensitive materials will generate considerable progress in measuring the usefulness of fragmentation, pullout, and indentation tests. The group also encourages the development of optical and other methods of obtaining axial fiber stress data for glass fibers, since they are the primary reinforcement used in commercial materials. 


\section{Needs of Industry Discussion Group Report \\ Dr. Thomas Donnellan - Northrop-Grumman \\ Dr. Anil Netravali - Cornell University}

The Needs of Industry discussion sessions, as was the case for the other three sessions, were held twice in two days. The discussion sessions were framed by posing four major questions to the attendees: 1)What information do the current interface strength measurement techniques provide? 2) How might these measurements be used in the future? 3) What are the dangers of over interpretation or improper analysis? and 4) What are the critical research questions and opportunities related to techniques and use? In the discussions, these questions were addressed but with significant overlap.

In answering the first question, the attendees felt that the information obtained from the numerous proposed interface characterization tests is different. This is due to different specimen geometries and the resulting different stress fields, and also because the mode of failure may be different in each test. As a result, the value obtained for interfacial shear strength (IFSS) in any of these tests is typically different. In general, the information obtained from tests is also material property dependent. There are also limits for every test and sometimes these limits may be forgotten. The industrial participants expressed a strong need for the research community to improve the fundamental understanding of the test measurements, the value of IFSS, and the importance of IFSS to composite properties. This fundamental understanding of interface contribution should be useful in optimizing the composite design and performance for various applications.

A number of researchers have found limited qualitative correlation between mechanical properties and the IFSS. However, quantitative correlation is still missing in most cases. Also, it has been found that extrapolation of these relationships to other fiber-matrix systems is very tenuous. Furthermore, systematic changes within a material system combination have been found to produce different trends when compared among techniques. Data reduction schemes in some cases may be based on correct assumptions, however, they certainly are too complex. As a result of these issues, the techniques are not used as a primary materials characterization tool. Most attendees felt that we have come a long way in theoretical understanding, data reduction, and simulations. However, they also felt that we have to go much further in developing theoretical analysis that is simple to understand and easy to apply.

At present, industry researchers use some of these tests depending upon their familiarity with the tests and the usefulness of the test to their applications. However, since every test gives different information and different IFSS value, it is generally necessary to conduct two or more different tests to obtain all the necessary information. This is very time consuming and costly, and the industry would prefer to have a test that is easy to perform and requires less time for use as a screening test in their effort to develop materials, surface treatments, and coupling agents. It must be remembered that new characterization techniques must either provide better information on material behavior or must provide similar information to conventional techniques, but do it more quickly or at lower cost.

One of the major points raised during the discussion was that most research to date has been conducted using a limited number of thermoset resins; mostly epoxies such as Epon 828 cured with mPDA and some other curing agents. However, the industry has been using 
a broad range of resin materials that include thermosets and thermoplastic resins. In fact, a great deal of the resins used in the industry are thermoplastic including polypropylene, nylons etc. and very little research has been done on these systems. It was suggested that more efforts be spent in understanding thermoplastic resins and their interactions with fibers. Establishing micro/macro composite correlation for a range of materials; especially thermoplastics would go a long way in helping the industry.

Another important issue raised during the discussion was the need to develop a technique to test the durability of composites and interfaces. At present, no micro-mechanics based tests are available for testing the durability of the interfaces. Most participants agreed that this is a difficult issue since the test may have to be conducted in-situ. The issue is further complicated by the varied environmental conditions such as moisture, temperature, and mechanical stresses to which composites are exposed. There is clearly a need for developing accelerated test procedures for composite testing to understand long term behavior.

In summary, the industry would appreciate a test that is simple, standardized, reliable, inexpensive, rapid, and yields critical information. Obviously we are far from this ideal test and our work is cut out for the next few years. With an improvement in the understanding of the tests that are available, and the influence of material and process effects on measurements made with the techniques, the potential for development of rapid material screening tools is a reasonable initial goal. 


\section{Results from Questionnaire \\ Dr. Richard Parnas - NIST}

The following is the compilation of the workshop questionnaire results. In ranking the responses to the questions, the votes were summed for rank 1 and 2 to provide a single number ranking of each item. From that, the key points from the questionnaire are:

1. Durability, mechanical properties, and cost were the overriding concerns of participants. However, it is puzzling that processing wasn't ranked higher since processing is a large fraction of the cost. This may be explained by the heavy concentration of aerospace applications where cost is important but speed is not. (Question \#3)

2. For implementing composites in commercial applications, durability and the establishment of acceptance criteria were most important, with manufacturing optimization a close second. (Question \#4)

3. For improving the usefulness of interfacial test methods, correlation of microtests with full composite behavior was most important, followed by test standardization. (Question \#7)

4. The SFFT is the most widely used test. (Question \#9)

5. None of the current tests are considered very useful although the indentation, SFFT, and short beam shear are marginal. (Question \#11) 


\section{Questionnaire $\mathbb{N}=2 \mathbb{1}$}

for

\section{Workshop on Micro-Mechanics Measurement Technologies}

for

Fiber-Polymer Interfaces

\section{When ranking answers 1 is most significant}

1. What is the focus of your interest in composites?

$\begin{array}{cl}-14 & \text { Industry } \\ -16 & \text { Academia }\end{array}$

- Experimentalist Theoretician

-15_ Government

5 _ Other, Specify__ Other Countries

2. What is the area of your composite application?

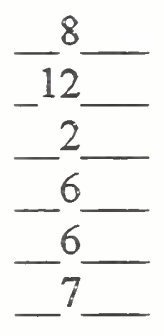

Automotive

Aerospace

Offshore

Construction

Marine

Other, Specify Circuit Boards, Appliances,_Sports, Dental

3. What factors are important for utilizing composites in your application?

123 rank

4_2_0_

$3+2-2$

6_6_2

$12^{2} 2$

$1+5+1$

470

752
Optimaum specific stiffness and strength

Weight savings

Mechanical properties

Maintenance and servicing requirements of the composite structure

Processing \& cycle time

Cost

Durability and long term performance of structure 
4. With respect to the implementation of composites in commercial applications, rank the following items in terms of their importance:

123 rank

5 4_4_ Optimization of composite manufacturing process

$38-2-$

0,33

$927^{-}$

$4+2-5$

Establishment of acceptance criteria for composites in various applications

Gaining approval from regulatory agency for use of composite structures

Long term durability of composite structures

Optimization for weight savings

5. How important is interfacial shear strength in your application

\begin{tabular}{ll}
$14-1$ & Very important \\
$-5-$ & Important \\
\hline 0 & Not Important
\end{tabular}

6. If interfacial shear strength is important, How well can we deal with the problem today?:

$\begin{aligned} 0 & \text { Very Well } \\ 5- & \text { Adequate } \\ -11- & \text { Not so adequate } \\ 2- & \text { Inadequate }\end{aligned}$

7. If you think interfacial shear strength is important, rank the impact of the following factors on improving the usefulness of interfacial test methods on the composite manufacturing process

123 rank

10_4_2_ Correlation of micro test results with full composite behavior

3 - 4_2 Improve micro test analysis methods

3 - 4_

$4-55^{-} \quad$ Standardization of the test

Other, Specify

8. What are the 1 or 2 most important shortcomings of the current models.

Lack of correlation with composite properties

- Tests are difficult and time consuming

Include effects of processing my making in situ measurements on full composite

_Need tests suitable for all materials, not just model materials

_Lack of data for statistical analysis

- Tests aren't̀ comparable

_Analysis lacking important effects such as matrix nonlinearity 
9. Do you currently use an interfacial shear strength measurement technique?

14___ Yes, please specify test and state why you chose this test

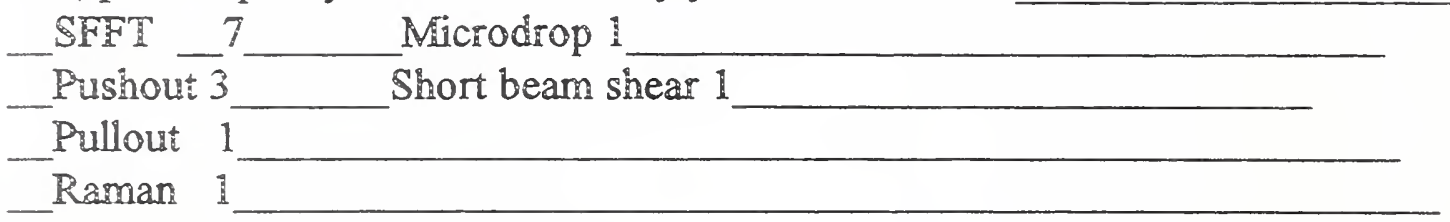

- N No, why?

Too difficult

Not well correlated to full composite

Measure bulk properties instead

10. In 5-10 years do you see people using micro tests in industry?

9 Y Yes, please elaborate on how you see the data being used

6 __ No, please elaborate __ Too difficult

Contract to fed labs or universities

11. Rank the following interfacial shear strength techniques based on their usefulness

123 rank

1 2_2

Bead Pull-Off Test

011

$33-1$

Atomic Force Microscopy (AFM)

3 - 1

124

420

Indentation Test

Single \& Multiple Fiber Fragmentation Tests

Single Fiber Pull Out / Push Out Tests

Short Beam Shear Test

8_ ${ }^{-} 0_{-} \quad$ None of the above tests are very good 


\section{FINAL PARTICIPANTS LIST}

\section{MICRO-MECHANICS MEASUREMENT TECHNOLOGIES FOR FIBER-POL YMER INTERFACES \\ National Institute of Standards and Technology \\ Gaithersburg, Maryland}

May 28-30, 1997

Mohamed Abdallah

Hexcel Corporation

P.O. Box 18748

Code 8186

Salt Lake City, UT 84118-0748 USA

Telephone: 801/251-3539

EMAIL: hexlnet.slc.mabdala@hexal.attmai

Maher Amer

Wright State University

Dept. of Mechanical \& Materials Eng.

3640 Colonel Glenn Highway

Dayton, OH 45435-0001

Telephone: 937/775-5095

FAX: 937/775-5009

Joseph Antonucci

NIST

Bldg. 224, Rm. A143

Gaithersburg, MD 20899-0001 USA

Telephone: 301/975-6726

EMAIL: joseph.antonucci@nist.gov

Paul Armistead

NRL

NRL Code 6120

Washington, DC 20375

Telephone: 202/767-6345

Fax: 202/767-0594
Maria Carmen Bautista

C/Lopez de Hoyos 351, 3C

28043 Madrid

Spain

Gerhard Burki

EMPA

Feuerwerkerstrasse 39

CH-3602 Thun

Telephone: 413/322-84626

Fax: 413/322-84604

EMAIL: gerhard.buerki@empa.ch

Leif Carlson

FAU

Dept. Of Mech. Eng.

Boca Raton, FL 33431 USA

Telephone: 561/367-3421

Yi-Chao Chen

University of Houston

Dept. Of Mech. Eng.

Houston, TX 77204 USA

Telephone: 713/743-4533

Fax: 713/743-4503

EMAIL:chen@uh.edu

Martin Chiang

NIST

Bldg. 224, Rm. A209

Gaithersburg, MD 20899-0001 USA

Telephone: 301/975-5186

Fax: 301/975-4977

EMAIL:martin.chiang@nist.gov 
Chia-Te (Rafael) Chou

Clark Schwebel Tech. Fab.

2200 S. Murray Ave.

P.O. Box 2627

Anderson, SC 29622 USA

Telephone: 864/260-3200

Roger Clough

NIST

Bldg. 223, Rm. A167

Gaithersburg, MD 20899-0001

Telephone: 301/975-6841

EMAIL: roger.clough@nist.gov

Harini Dasarathy

Hexcel Corporation

3300 Mallard Fox Dr.

P.O. Box 2247

Decatur, AL 35609 USA

Telephone: 205/340-4029

Fax: 205/340-4030

EMAIL: dasharin@hotmail.com

Anthony DiBenedetto

University of Connecticut

Institute of Material Science

U-136

Storrs, CT 06269 USA

Telephone: 860/486-5252

Fax: 860/486-4745

EMAIL: adibened@mail.ims.uconn.edu

Tom Donnellan

Northrop Grumman

Bethpage, NY 11714

Telephone: 516/575-5474

Fax: 518/346-3171

EMAIL:

tom_donnellan@atdc.northgrum.com

Lawrence Drzal

Michigan State University

2100 Engineering Bldg.

East Lansing, MI 48824 USA

Telephone: 517/353-7759

Fax: 517/432-1634
David Dwight

Owens Corning

2790 Columbus Rd., Route 16

Granville, OH 43023-1200 USA

Telephone: 614/321-7511

Fax: 614/321-8023

EMAIL: david.dwight@owenscorning.com

Bruno Fanconi

NIST

Bldg. 224, Rm. A309

Gaithersburg, MD 20899-0001 USA

EMAIL: bruno.fanconi@nist.gov

Andreas Hampe

BAM

Lotzestr

D-12205-Berlin GERMANY

Telephone: 493/08104160

Fax: 493081041607

EMAIL: andreas.hampe@bam.de

Pedro Herrera-Franco

Centro de Investigacion

Hda Xcumpich KM 7

Antigua Carr A Prog

Merida Yucatan, 97310 MEXICO

pherrera@cicy.cicy.mx

Henjen Ho

Michigan State University

2100 Engineering Bldg.

Comp. Mat. \& Struct. Ct.

East Lansing, MI 48824 USA

Telephone: 517/353-5411

Fax: 517/432-1634

EMAIL: hohenjen@egr.msu.edu

Gale Holmes

NIST

Bldg. 224, Rm. B108

Gaithersburg, MD 20899-0001 USA

Telephone: 301/975-5280

Fax: 301-975-4932

EMAIL: gale.holmes@nist.gov 
Donald Hunston

NIST

Bldg. 224, Rm. B108

Gaithersburg, MD 20899-0001 USA

Telephone: 301/975-6837

Fax: 301/975-4932

EMAIL: donald.hunston@nist.gov

Gerhard Kalinka

BAM Fed. Inst. Mat. Res. \& Ts.

Unter Den Eichen 87- Lab VI.21

Berlin, D12209 GERMANY

Fax: 517/432/1634

EMAL:kalinkag@egr.umsu.edu

John Maguire

Southwest Research Inst.

6220 Culebra Rd.

San Antonio, TX 78228 USA

Telephone: 210/522-3560

Fax: 210/522-5122

EMAIL:jmaguire@swri.edu

Guangzhao Mao

Wayne State University

5050 Anthony Wayne Dr., Rm. 1109

Detroit, MI 48202 USA

Telephone: 313/577-3804

Fax: 313/577-3810

Cary Marzinsky

Exxon Research \& Eng.

Rt. 22E, LC280

Annandale, NJ 08801 USA

Telephone: 908/730-2837

Fax: 908/730-3355

EMAIL: emarzi@ereng.com

Walter McDonough

NIST

Bldg. 224, Rm. B108

Gaithersburg, MD 20899-0001 USA

Telephone: 301/975-3661

Fax: 301/975-4932

EMAIL: walter.mcdonough@nist.gov
Ray Meilunas

Northrop Grumman

Mail Stop A01-26

Bethpage, NY 11714 USA

Telephone: 516/346-6247

Fax: 516/346-3670

Bernard Miller

TRI/Princeton

Box 625

Princeton, NJ 08542 USA

Telephone: 609/924-3150

Fax: 609/924-7149

Zenichi Miyagi

National Research Lab. Of Met. (NRLM)

1-1-4 Umezono

Tsukuba, Ibaraki, 305 JAPAN

Telephone: 029/854-4043

Fax: 0298-54-4061

EMAIL:miyagi@nnlm.go.jp

Russ Mueller

Exxon Research \& Eng.

Rt. 22E, LC172

Annandale, NJ 08801 USA

Telephone: 908/730-2630

John Nairn

University of Utah

Salt Lake City, UT 84112 USA

Telephone: 801/581-3413

Fax: 801/581-4816

EMAIL:john.nairn@m.cc.u

Anil Netravali

Cornell University

Fiber Science Prog.

Ithaca, NY 14853-4401 USA

Telephone: $607 / 255-1875$

Fax: 607/255-1083

EMAIL:ann2@cornell.edu 
Steven Nutt

USC

Material Science

VHE-602

Los Angeles, CA 90089-0241 USA

Telephone: $213 / 740-1634$

Fax: 213/740-7744

EMAIL:nutt@usc.edu

Richard Parnas

NIST

Bldg. 224, Rm. B108

Gaithersburg, MD 20899-0001 USA

Telephone: 301/975-5805

Fax: 301/975-4932

EMAIL: richard.parnas@nist.gov

Richard Peterson

NIST

Bldg. 224, Rm B108

Gaithersburg, MD 20899-0001 USA

Telephone: 301/975-4441

Fax: 301/975-4932

EMAIL: richard.peterson@nist.gov

Fred Phelan

NIST

Bldg. 224, Rm B108

Gaithersburg, MD 20899-0001 USA

Telephone: 301/975-4441

Fax: 301/975-6761

EMAIL: frederick.phelan@nist.gov

S. Leigh Phoenix

Cornell University

321 Thurston Hall

Ithaca, NY 14853 USA

Telephone: $607 / 255-8818$

Fax: 607/255-2011

EMAIL:slp6@cornell.edu
Eric Pohl

Witco

777 Old Saw Mill River

Tarrytown, NY 10591 USA

Telephone: 914/784-4911

Fax: 914/784-4890

Kaushik Raghavan

NIST

Bldg. 224, Rm. B108

Gaithersburg, MD 20899-0001 USA

Michael Rich

Michigan State University

2100 Engineering Bldg.

Comp. Mat. \& Struct. Ct.

East Lansing, MI 48824 USA

Telephone: 517/353-4696

Fax: 517-353-1634

EMAIL: rich@egr.msu.edu

Nicola Richards

University of Florida

Dental Biomaterials

Box 100446

Gainesville, FL 32610-0446 USA

Telephone: 352/392-4351

Fax: 352/392-7808

EMAIL: nrichards@dental.ufl.edu

David Salem

TRI/Princeton

P.O. Box 625

Princeton, NJ 08562 USA

Telephone: 609/926-3150

Fax: 609/683-7189

EMAIL: dsalem@triprinceton.org

Linda Schadler

RPI

$1108^{\text {th }}$ St.

MRC

Troy, NY 12180-3590 USA

Telephone: 518/276-2022

Fax: 518/276-8554

EMAIL: schadl@rpi.edu 
Carl Schultheisz

NIST

Bldg. 224, Rm. A209

Gaithersburg, MD 20899-0001 USA

Telephone: 301/975-6847

EMAIL: carl.schultheisz@nist.gov

Leslie Smith

NIST

Bldg. 224, Rm. A309

Gaithersburg, MD 20899-0001 USA

EMAIL: leslie.smith@nist.gov

Michael Spaid

NIST

Bldg. 224, Rm. B108

Gaithersburg, MD 20899-0001 USA

Telephone: 301/975-6280

EMAIL:michael.spaid@nist.gov

Russell Stackpole

Touchstone Research Lab

Millenium Ctr.

Triadelphia, WV 26059 USA

Telephone: 304/547-5800

Fax: 304/547-4069

EMAIL:rps@gold.trl.com

Shaun Stephens

Boeing

P.O. Box 3707, MS 4T-80

Seattle, WA 98119 USA

Telephone: 206/655-9603

Fax: 206/662-1801

EMAIL: shaun.m.stephens@boeing.com

Tim Tufts

Ashland Chemical Company

5200 Blazer Parkway, DR-3

Dublin, OH 43017 USA

Telephone: 614/790-3772

Fax: 614/790-6106

EMAIL:

tim_tufts\%ashchem@notesgw.compuse
Sheldon Wesson

TRI/Princeton

601 Prospect Avenue

Princeton, NJ 08542 USA

Telephone: 609/683-3150

Fax: 609/683-7836

EMAIL:wesson@ynir.com

Carol Williams

NSWC-Carderock

Code 6553

9500 MacArthur Blvd.

West Bethesda, MD 20817-5700 USA

Robert Young

UMIST

Grosvenor St.

Manchester M1 7HS UK

Telephone: 44-161-2000

Fax: 44-161-2000-8871

EMAIL: robert.young@umist.ac.uk 



\section{APPENDIX}

NIST policy requires the use of SI units. However, the non-NIST contributions to this document are reported in the units used in the non-NIST work, which may not be SI. Results and conclusions should not be quoted without consulting the authors and obtaining approval.

Certain commercial materials and equipment are identified in this paper in order to specify adequately the experimental procedure. In no case does such information imply recommendation or endorsement by the National Institute of Standards and Technology, nor does it imply necessarily that the items are the best available for the purpose. 



\title{
OVERVIEW OF INTERFACE TESTING
}

\author{
Dr. Lawrence T. Drzal \\ Michigan State University \\ 2100 Engineering Building \\ East Lansing, MI 48824
}

\section{Slide No.}

3. One often neglected consideration in fiber-matrix interfacial measurements in the role of processing in performance.

5-6. Composite structural properties are dependent on lamina properties which do not explicitly include constituent properties. Even at the lamina level fiber and matrix properties are used to predict lamina properties but interfacial properties (e.g. adhesion) are not considered.

10. Fiber-matrix interface properties can have a major role in lamina properties not only in shear and transverse properties but also axial properties as the following examples show. Examples will be given using three composites systems fabricated from the same carbon fiber at the same volume fraction in the same epoxy matrix processed under identical conditions. The only difference is that the adhesion changes significantly from AU-4 to $\mathrm{AS}-4$ to $\mathrm{AS}-4 \mathrm{C}$ and the interfacial failure mode changes from interfacial to matrix.

11-22. Fiber dominated axial composite properties showing the effect of varying fiber-matrix adhesion and failure mode.

23-33. Fiber-Polymer Interface Dominated tests showing the effect of varying fiber-matrix adhesion and failure mode.

34. Interfacial failure mode can play as large a role as fiber-matrix adhesion.

35. Standard protocols exist (ASTM) for measuring laminate properties but do not exist for interfacial properties.

37-51. There are several direct microtests for measuring fiber-matrix adhesion involving the testing of a single isolated fiber.

52-60. There are several indirect microtests available for measuring fiber-matrix adhesion although they are not commonly used.

63-67. The methods of data analysis of each of the direct microtests differ and both a stress and energy approach are possible.

68. Beyond the issues related to how to interpret the micro test data are issues related to sample preparation, processing, testing procedures and measurement of interphase 
properties.

69. Care must be taken to eliminate voids at the fiber-matrix interface.

70. Coatings may create another interface due to coating/matrix incompatibility.

71-72. Failure modes may prejudice the data. Matrix cracking can cause premature sample failure.

73-74. Nearest neighbor interactions are important. Isolated single fiber tests do not have any neighboring fibers. When these fibers are present they can influence the stress distribution and adjacent fiber fractures.

74-76. Polymeric reinforcing fibers do not lend themselves to easy evaluation by most microtests since they fail in a fibrillar manner.

77-78. Sizings or finishes can create interphases having properties drastically different than the surrounding matrix.

79-80. Interphases must be characterized for their local material properties at the $10 \mathrm{~nm}$ level if a three predictive model which includes the fiber/matrix interface is ever to be developed and used.

81. The task of the workshop is to start the discussion as to what characteristics are to be measured and how. 

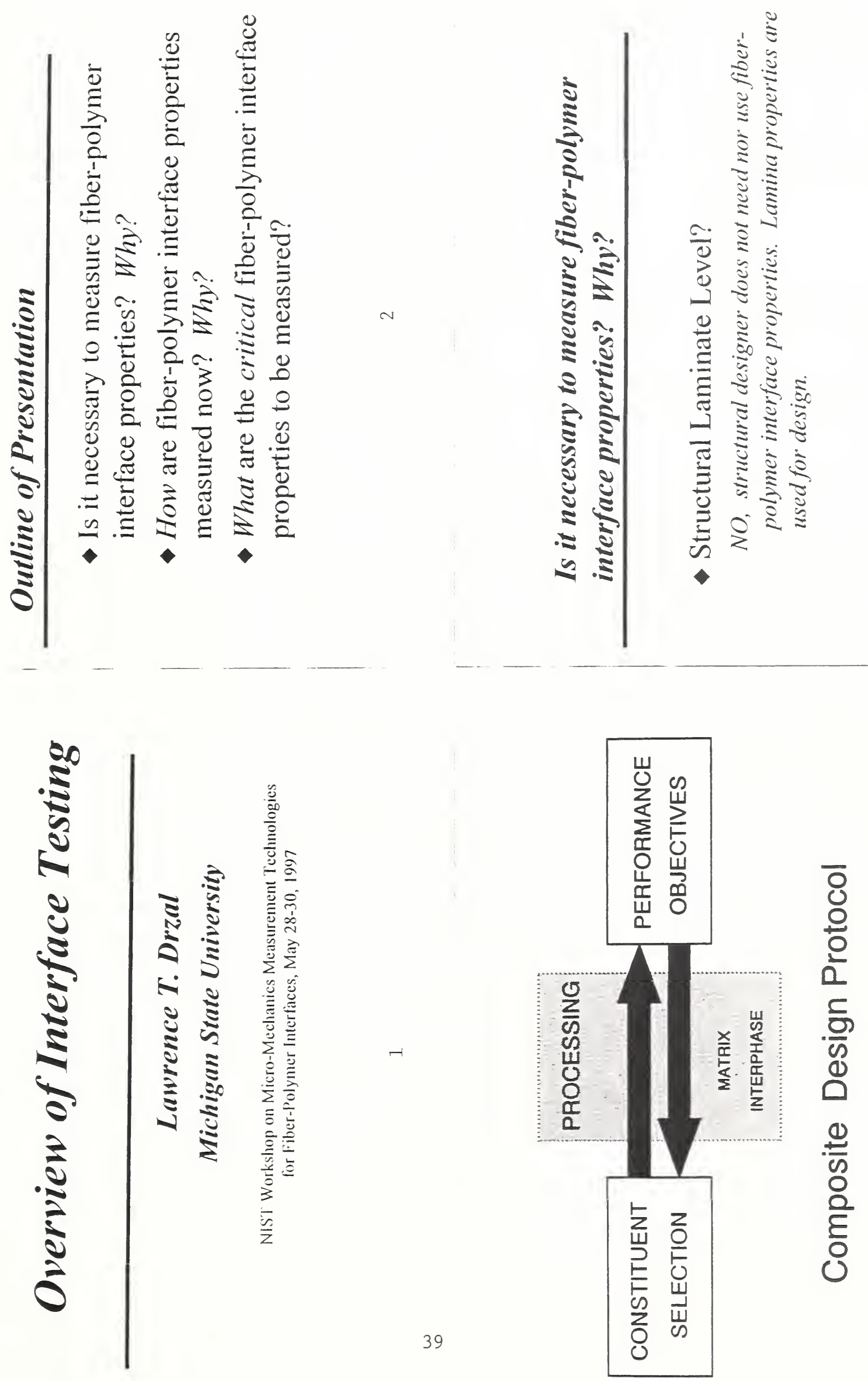

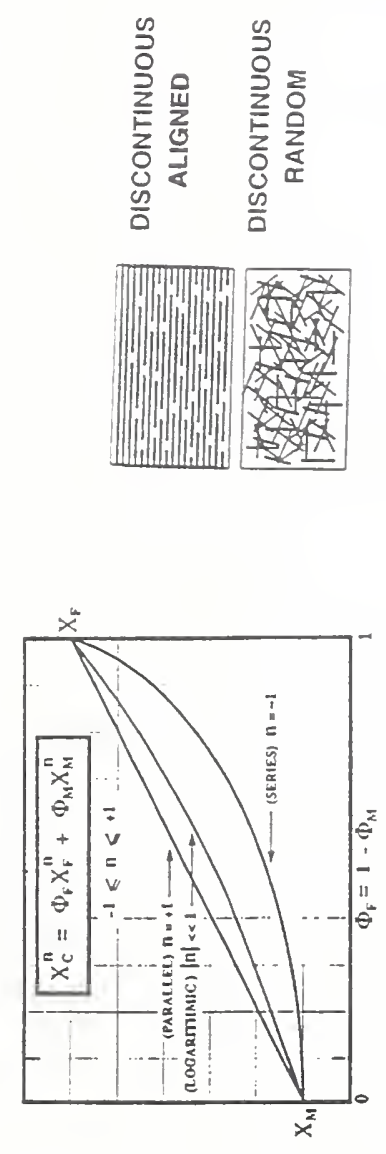

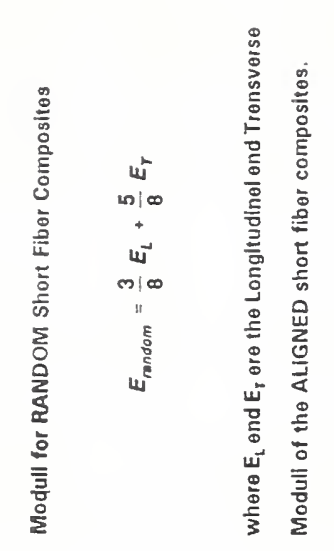

6

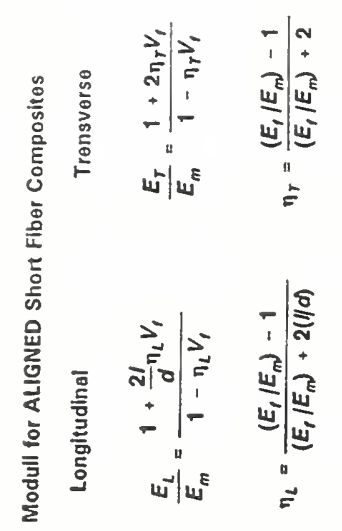

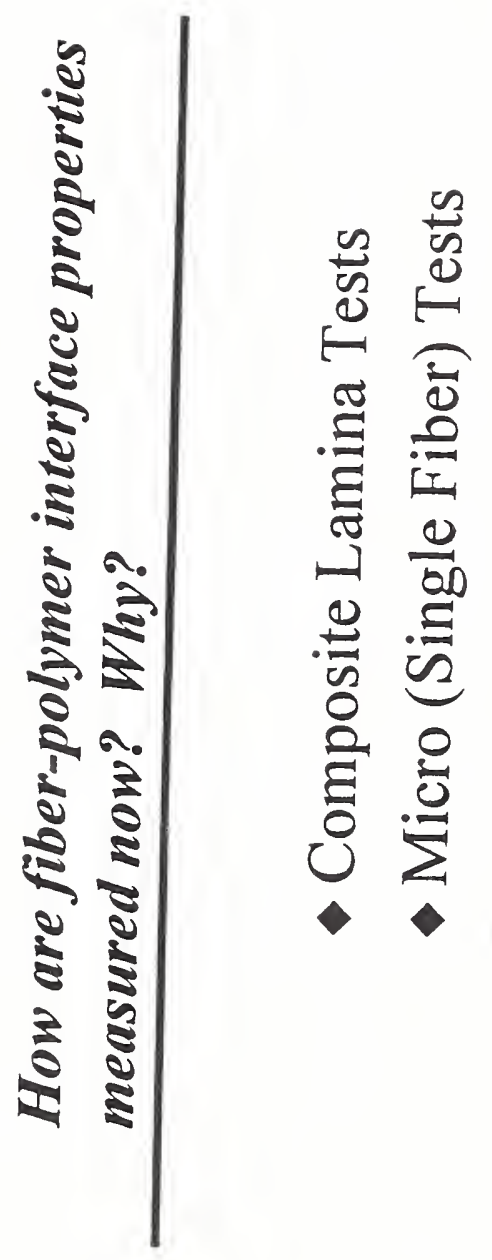

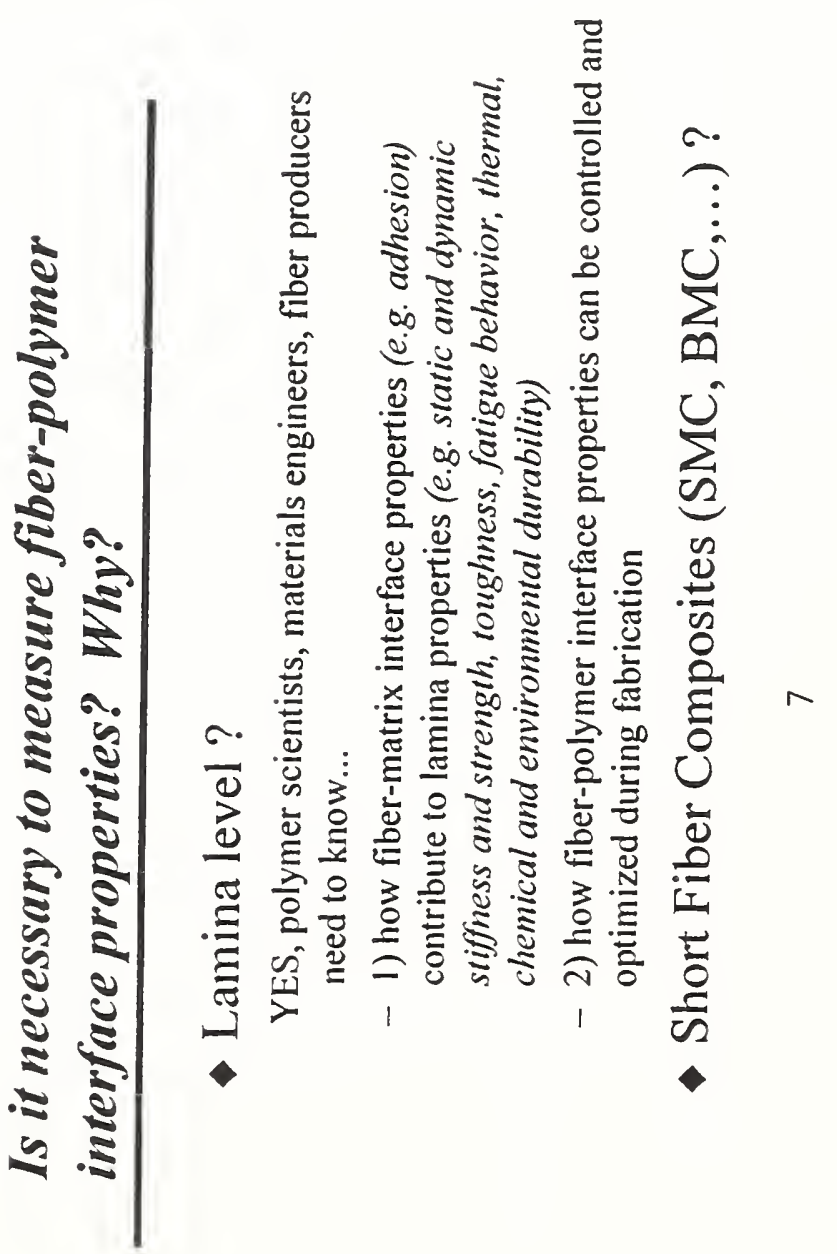



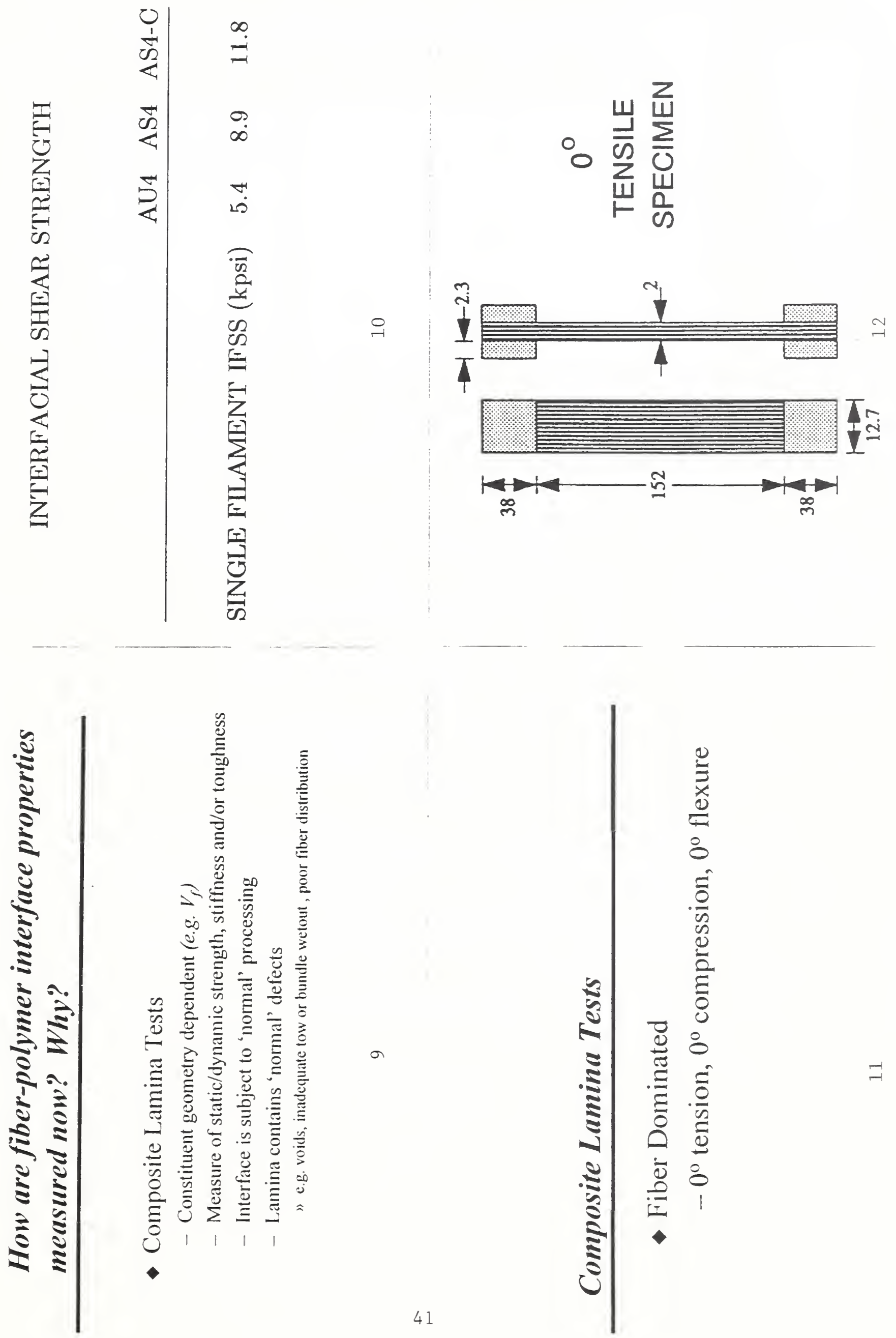

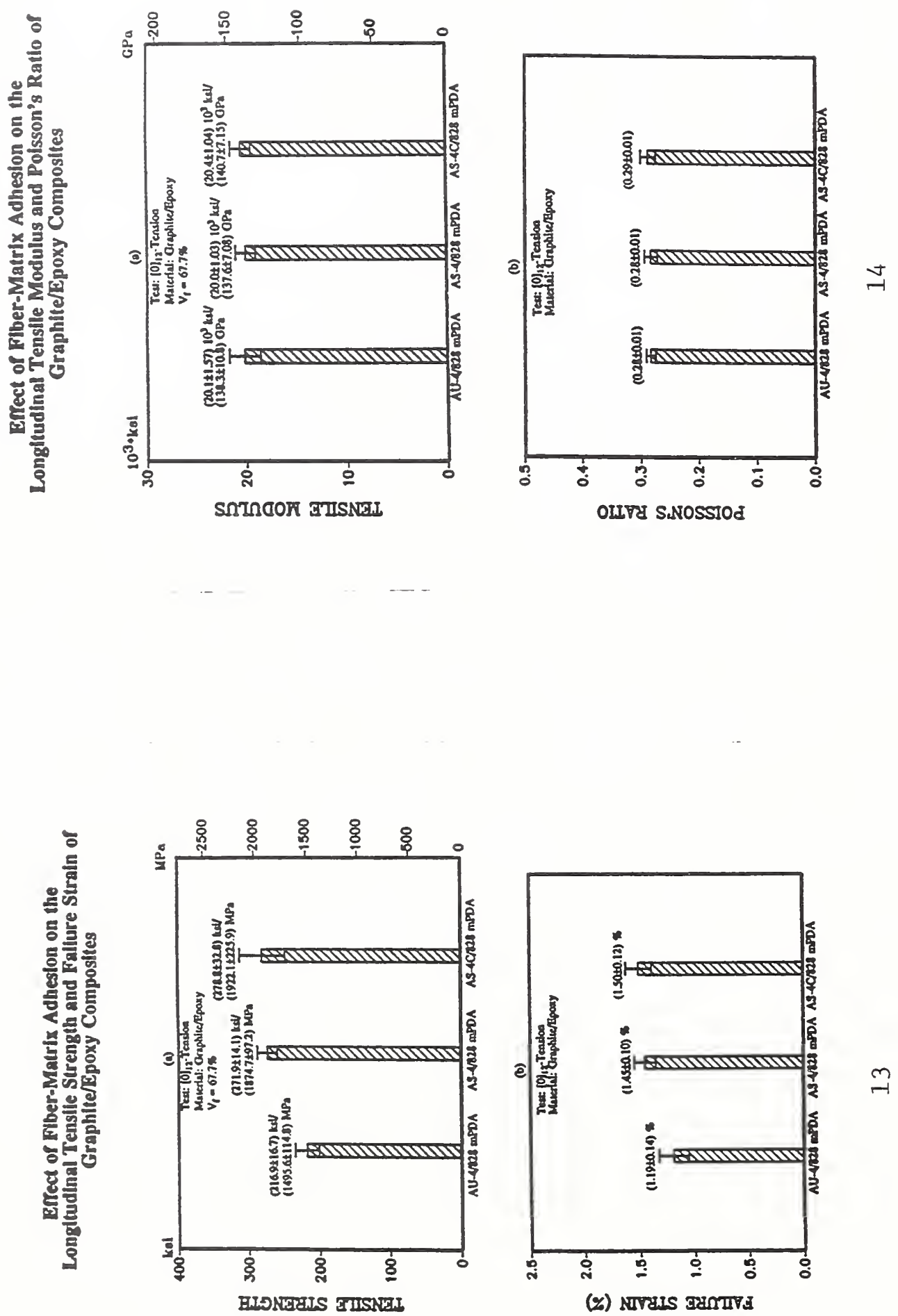

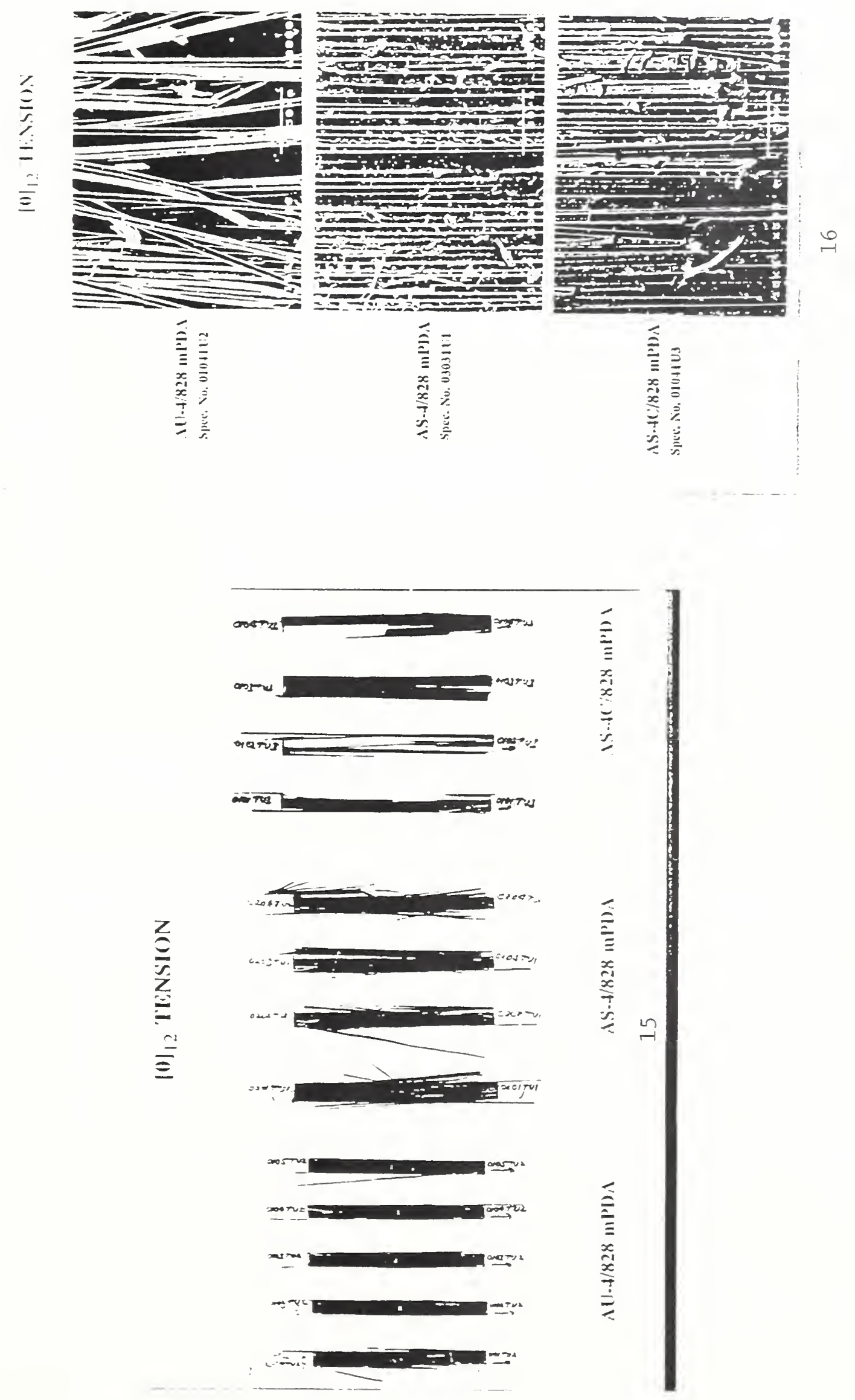

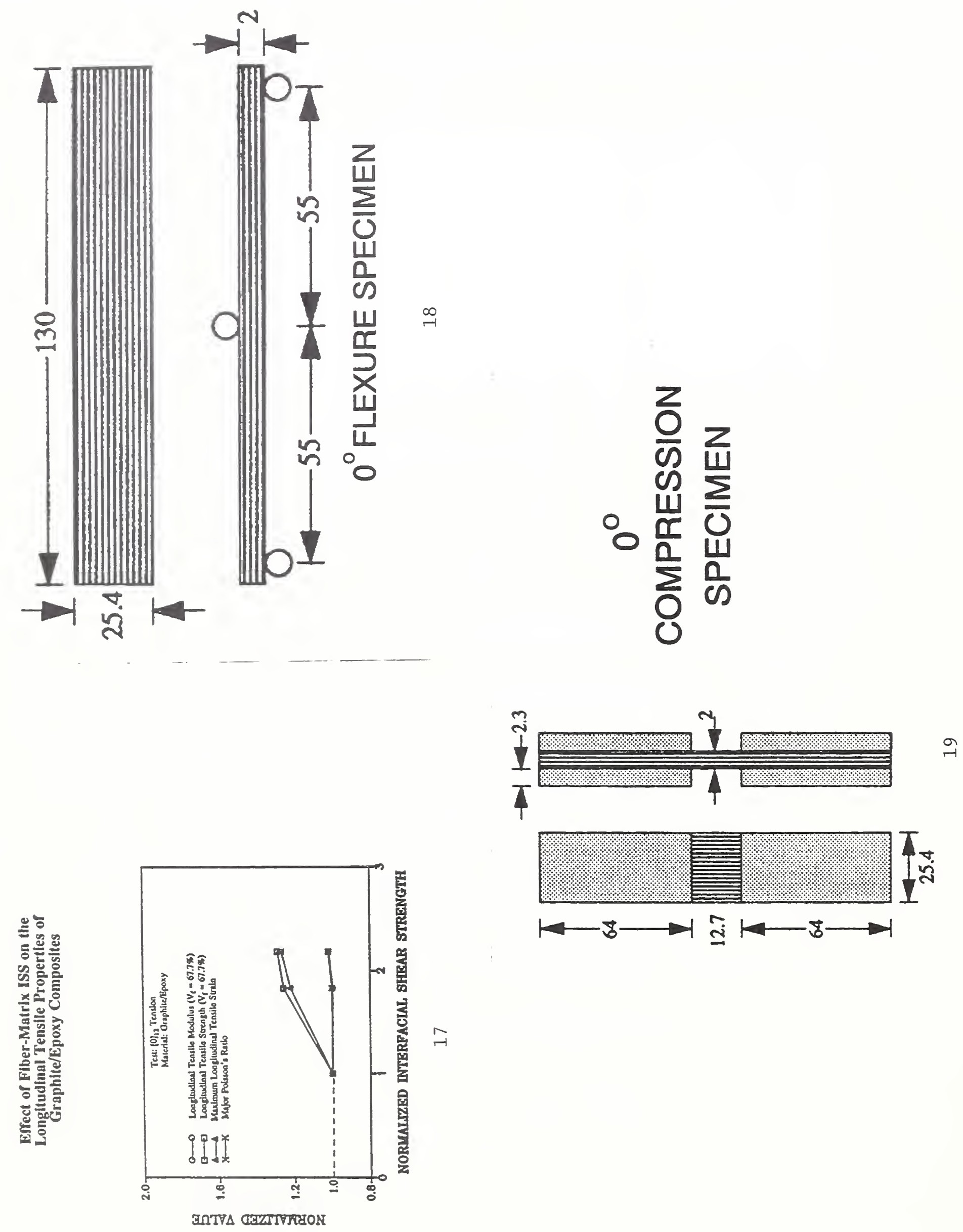


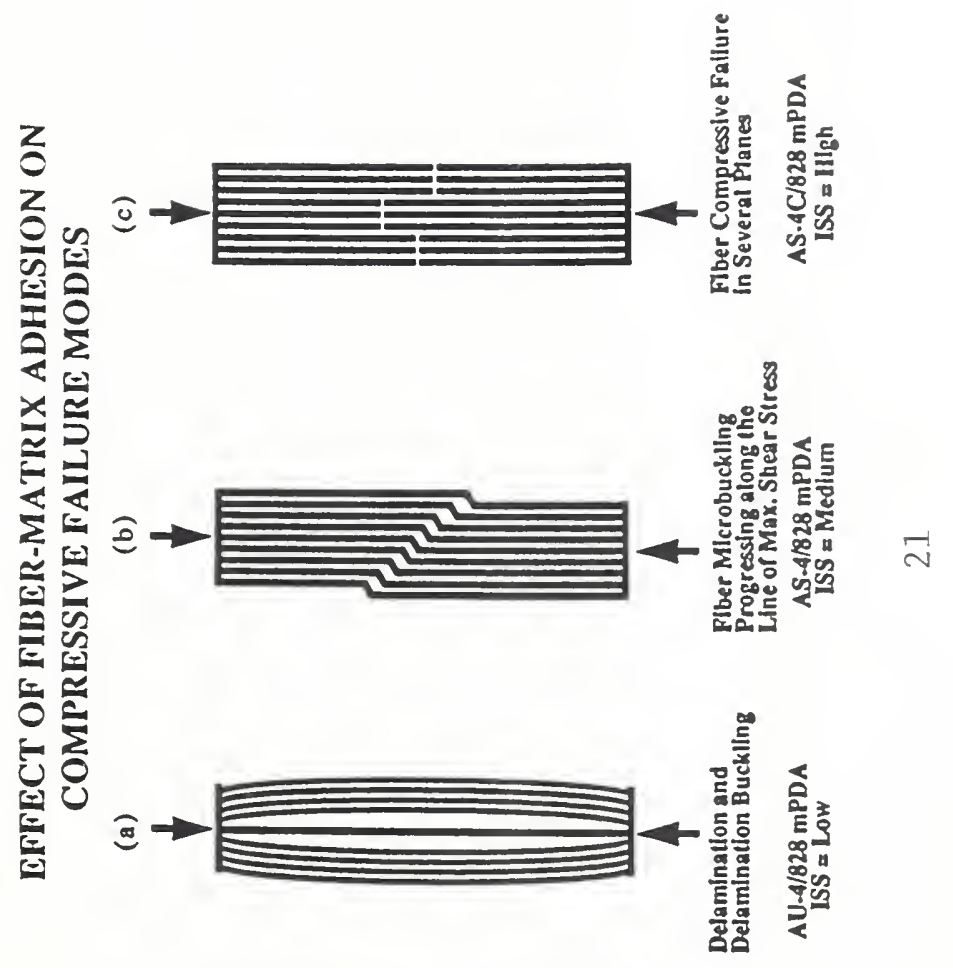

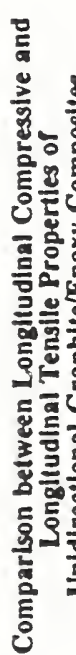
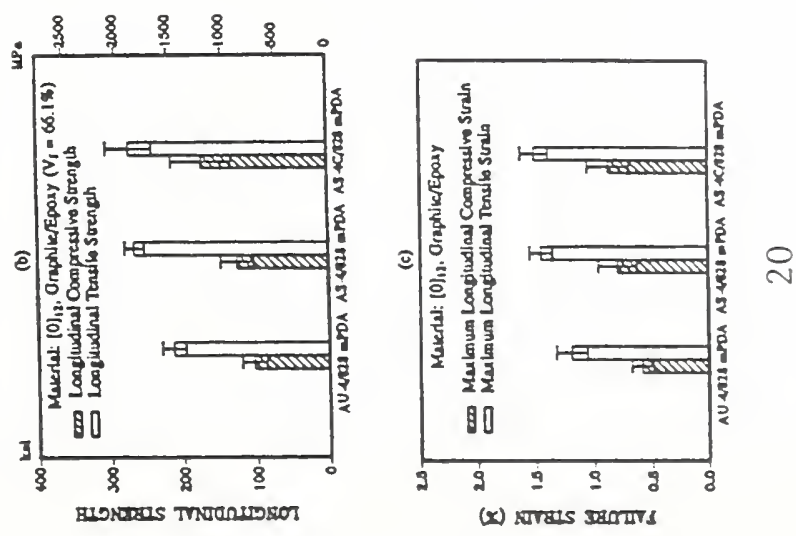

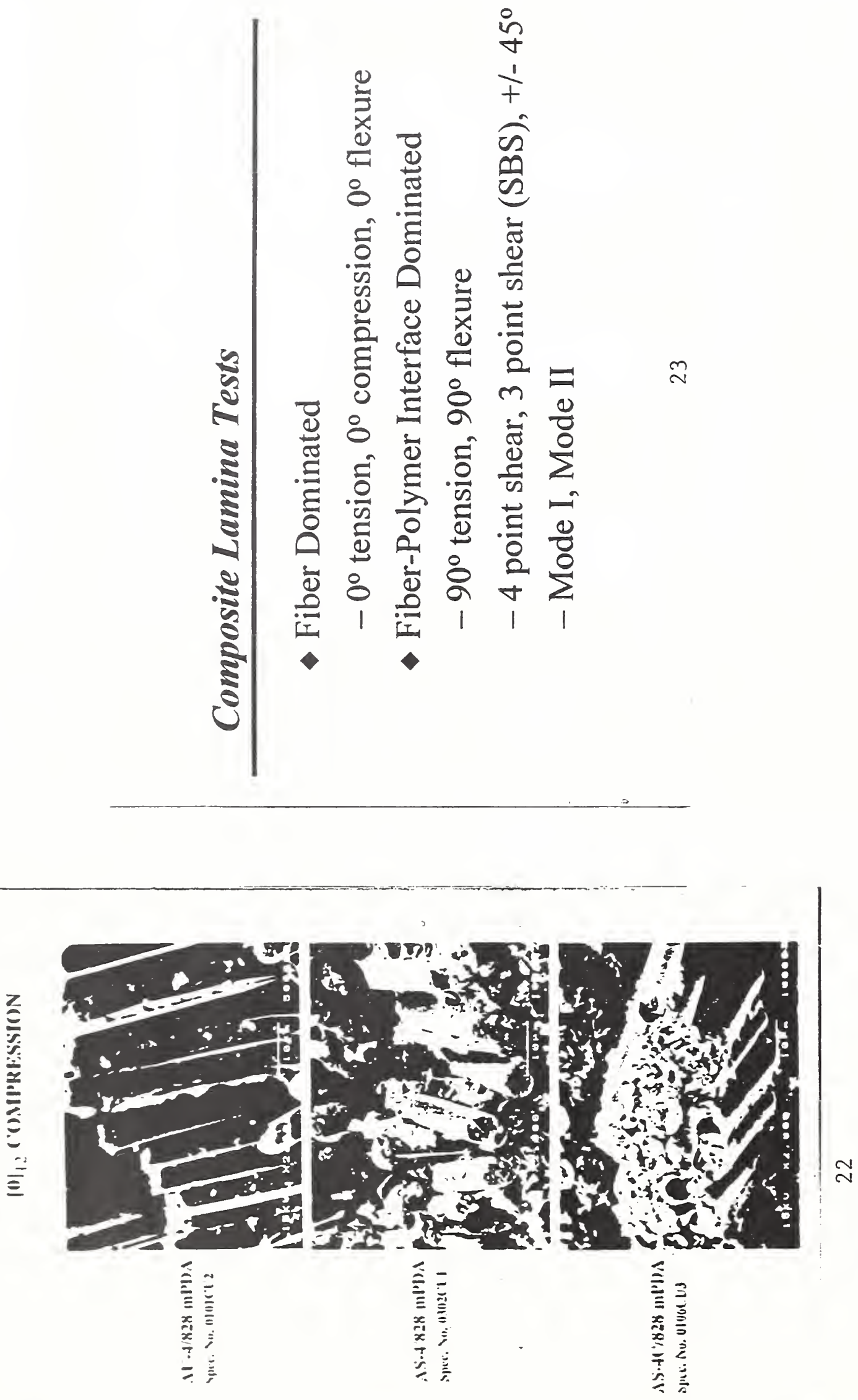

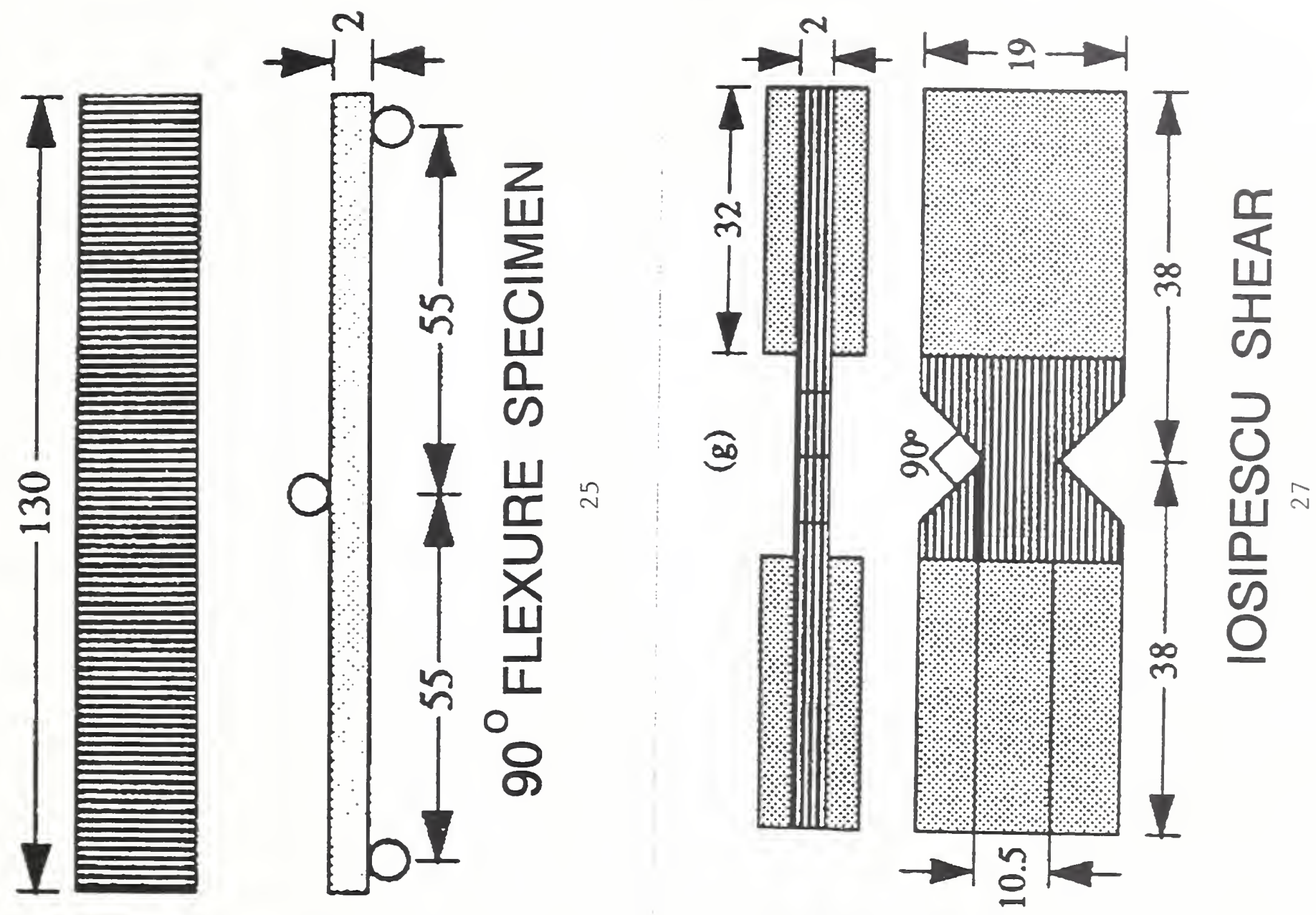

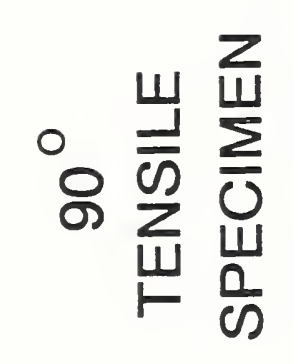
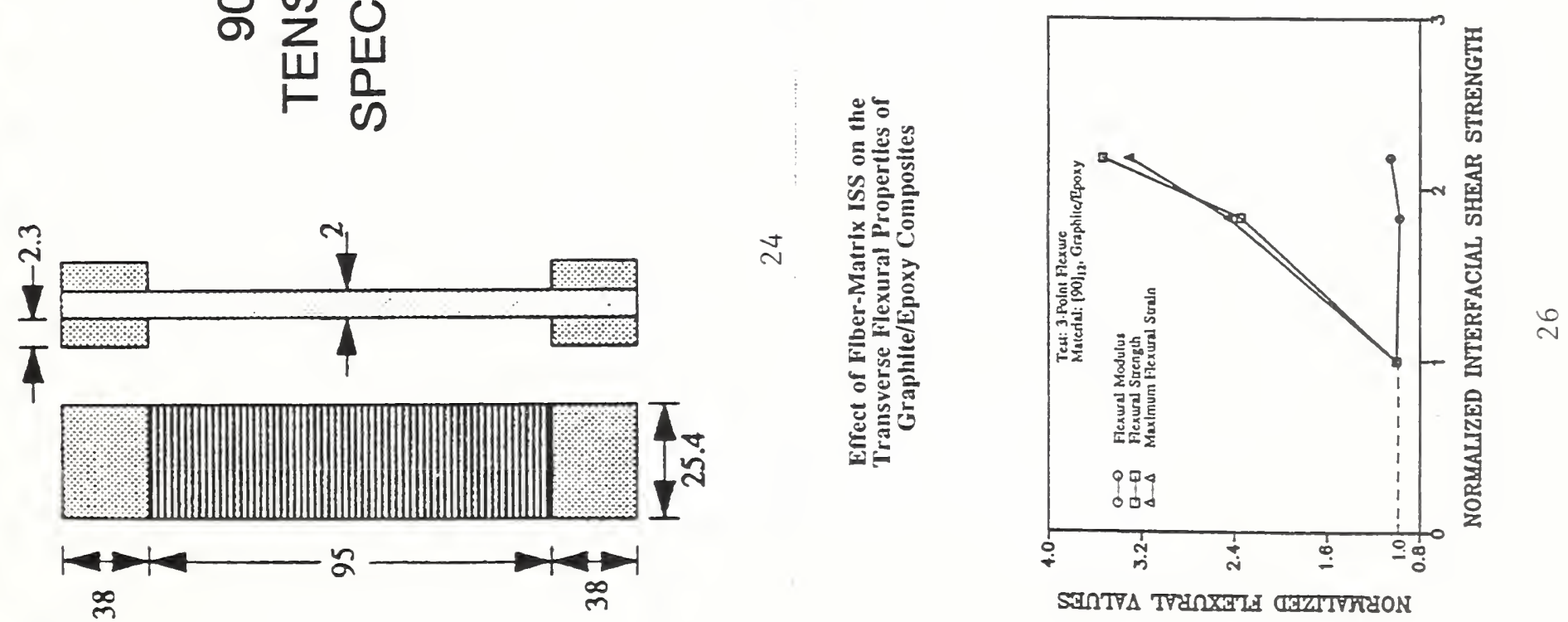


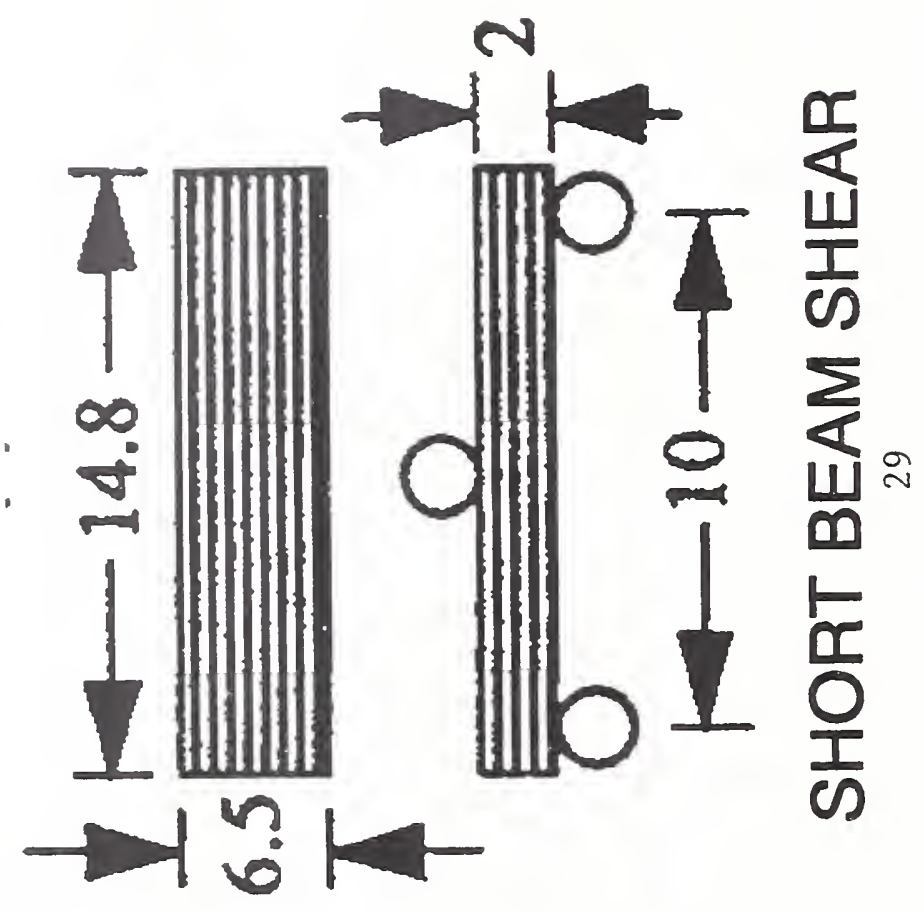

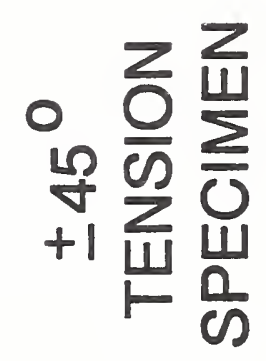

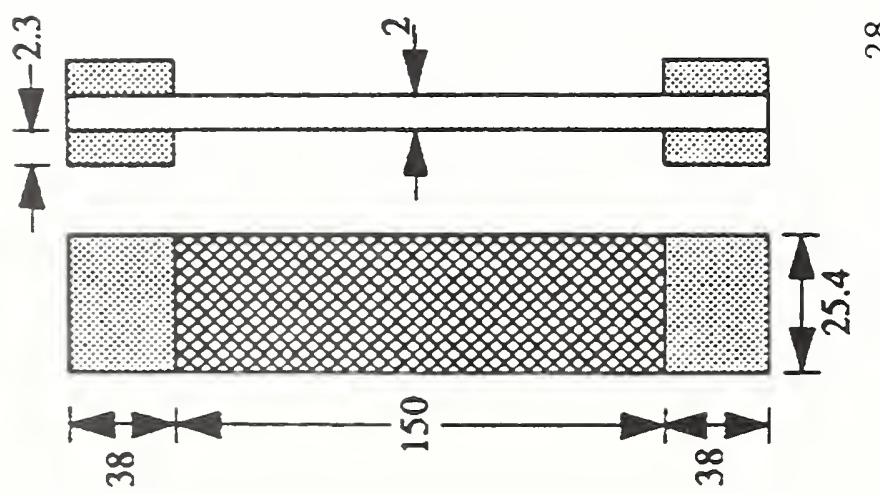



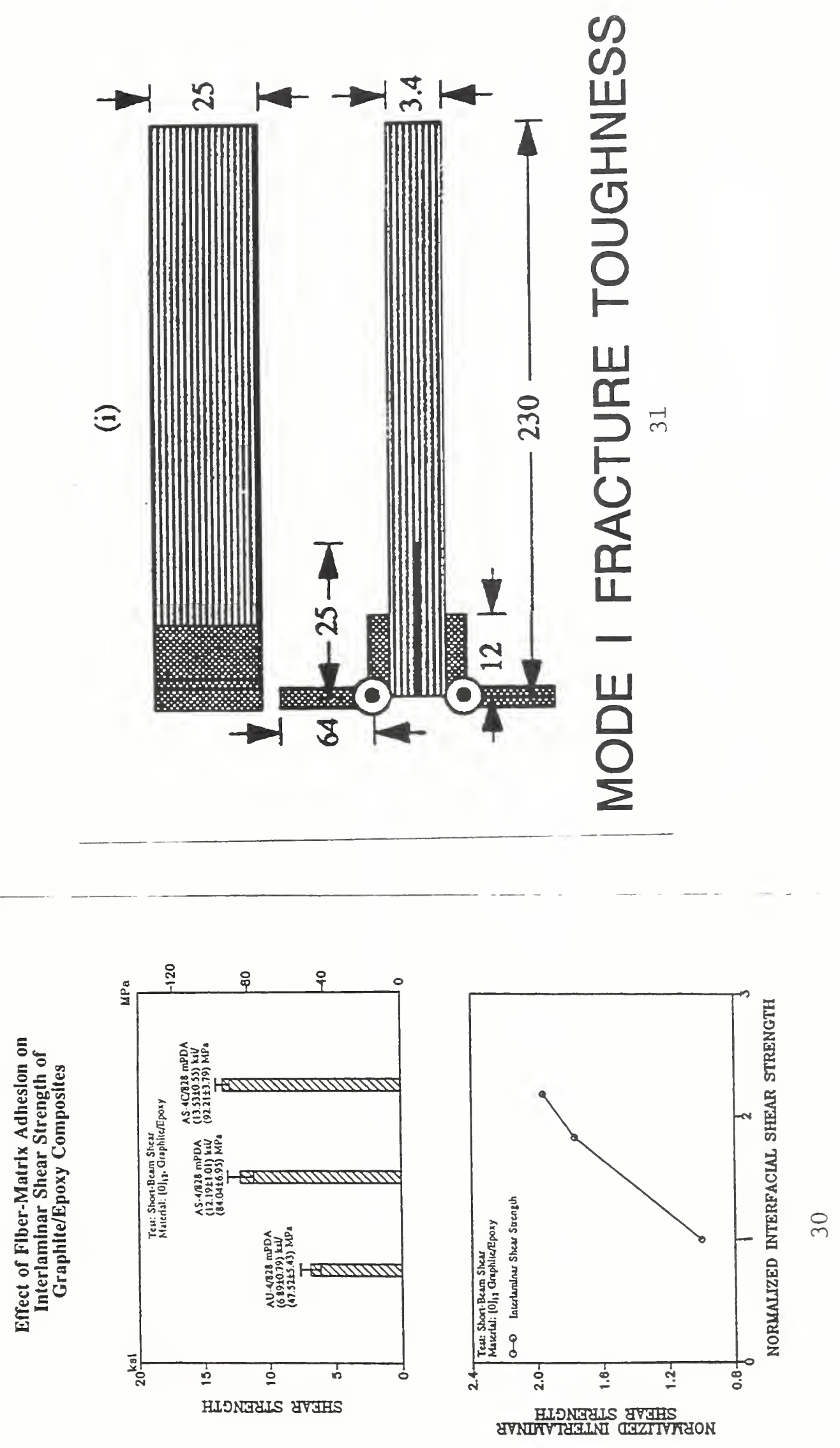

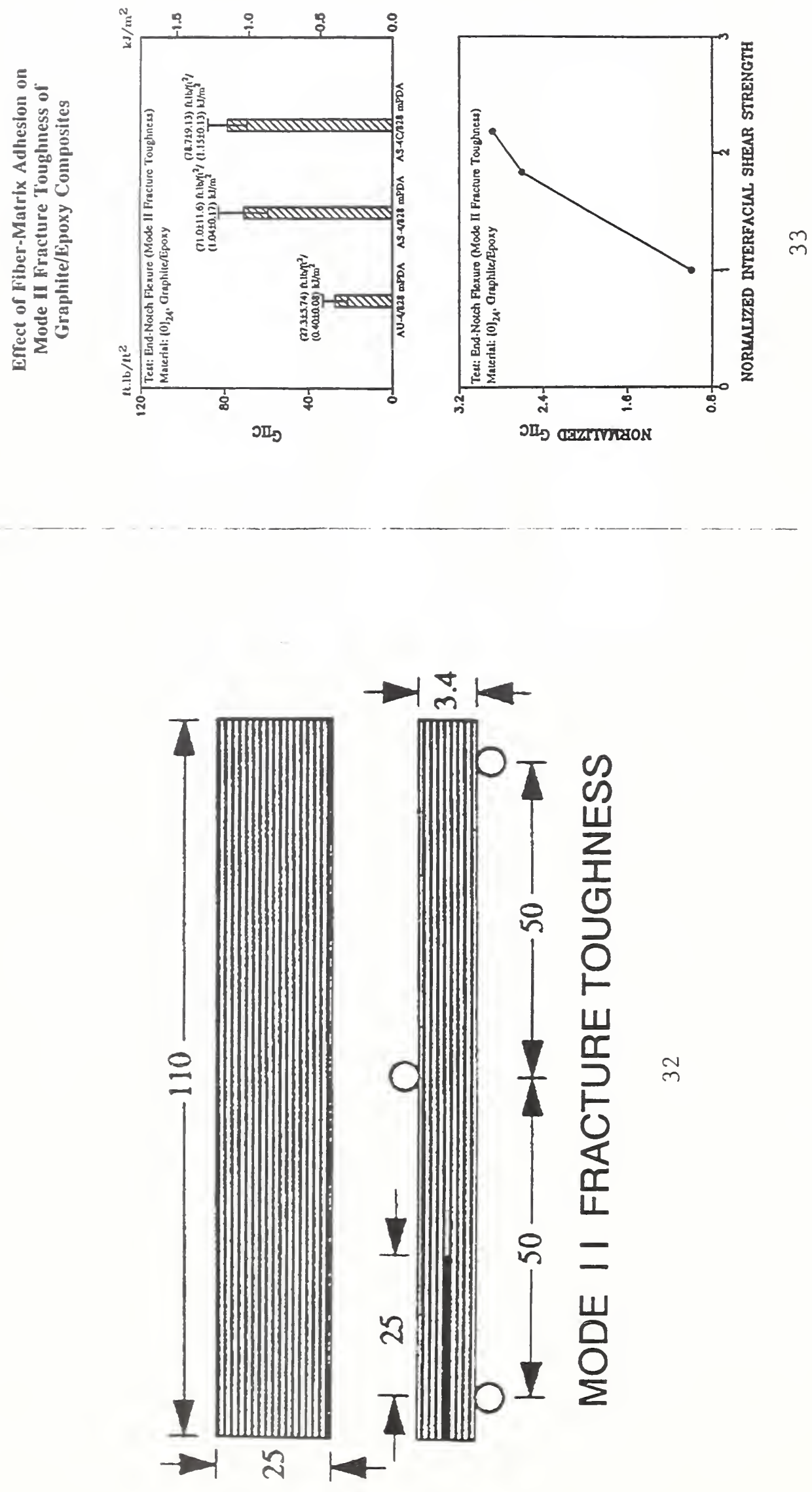

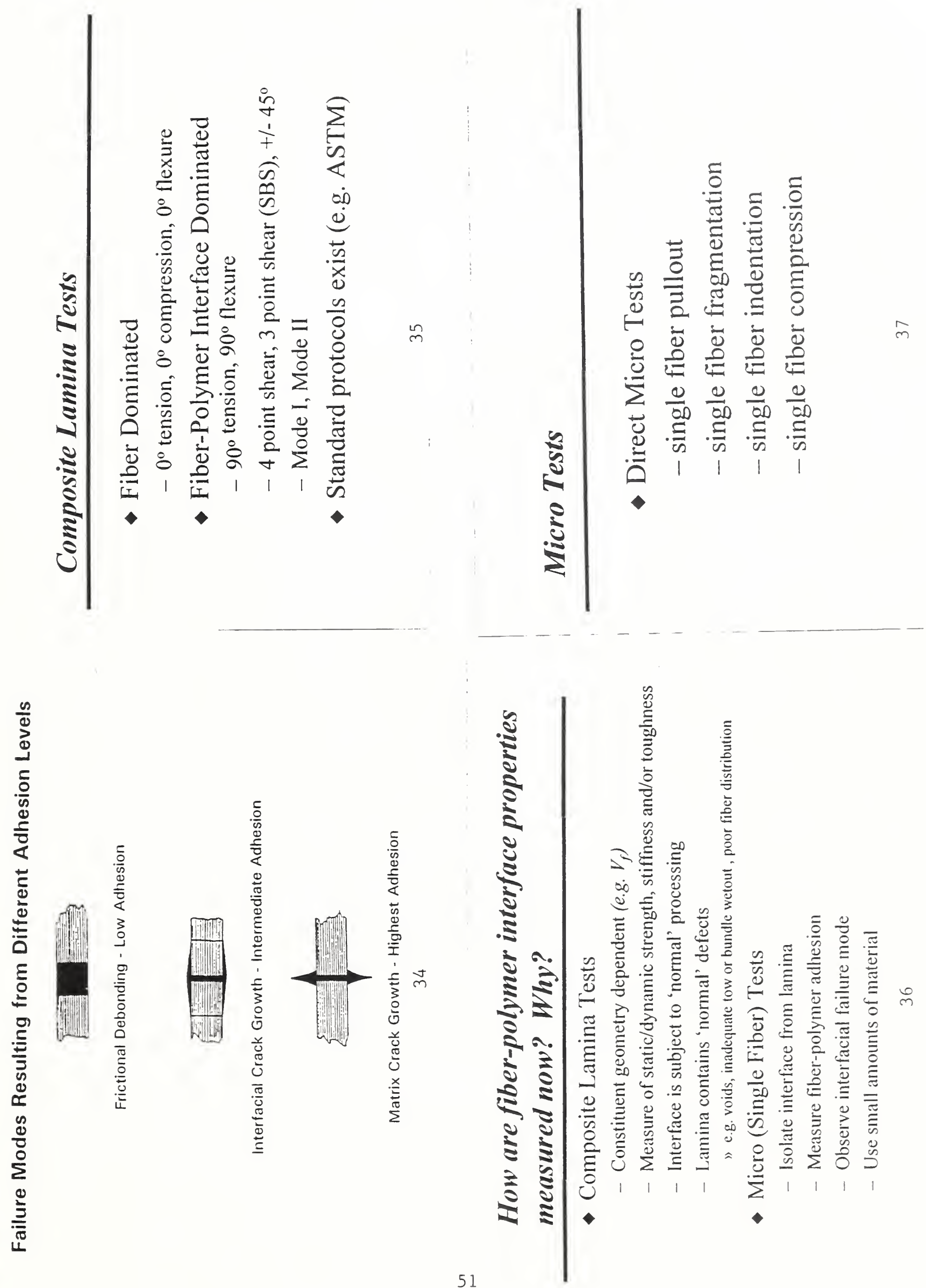

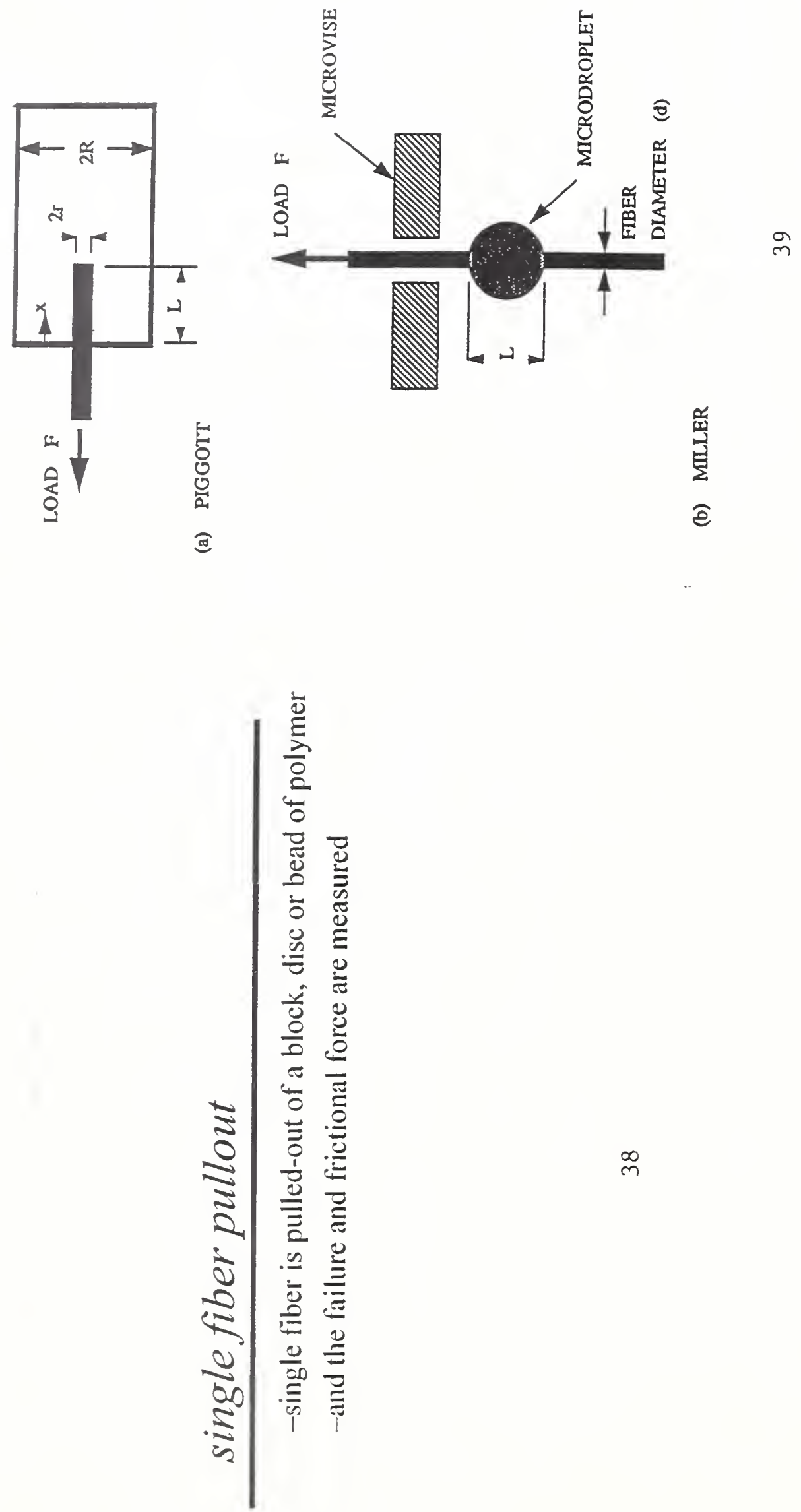

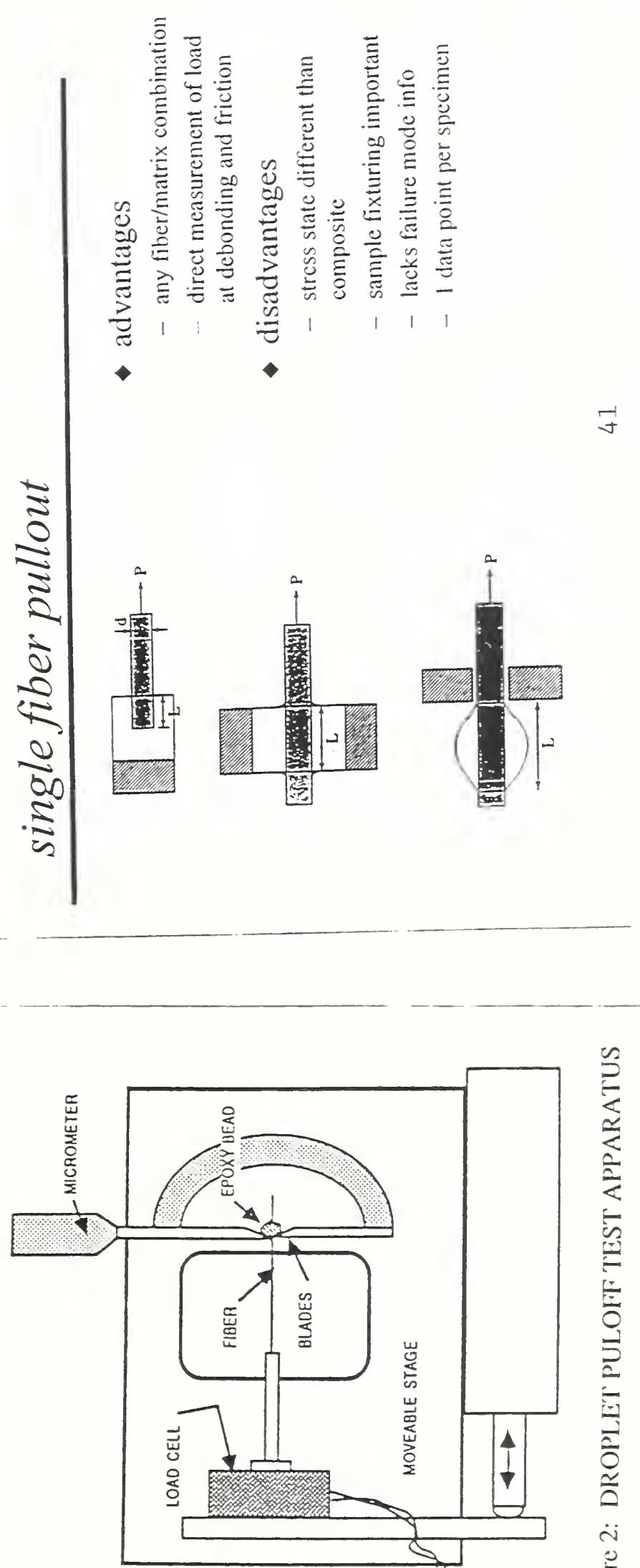

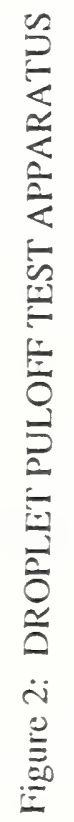




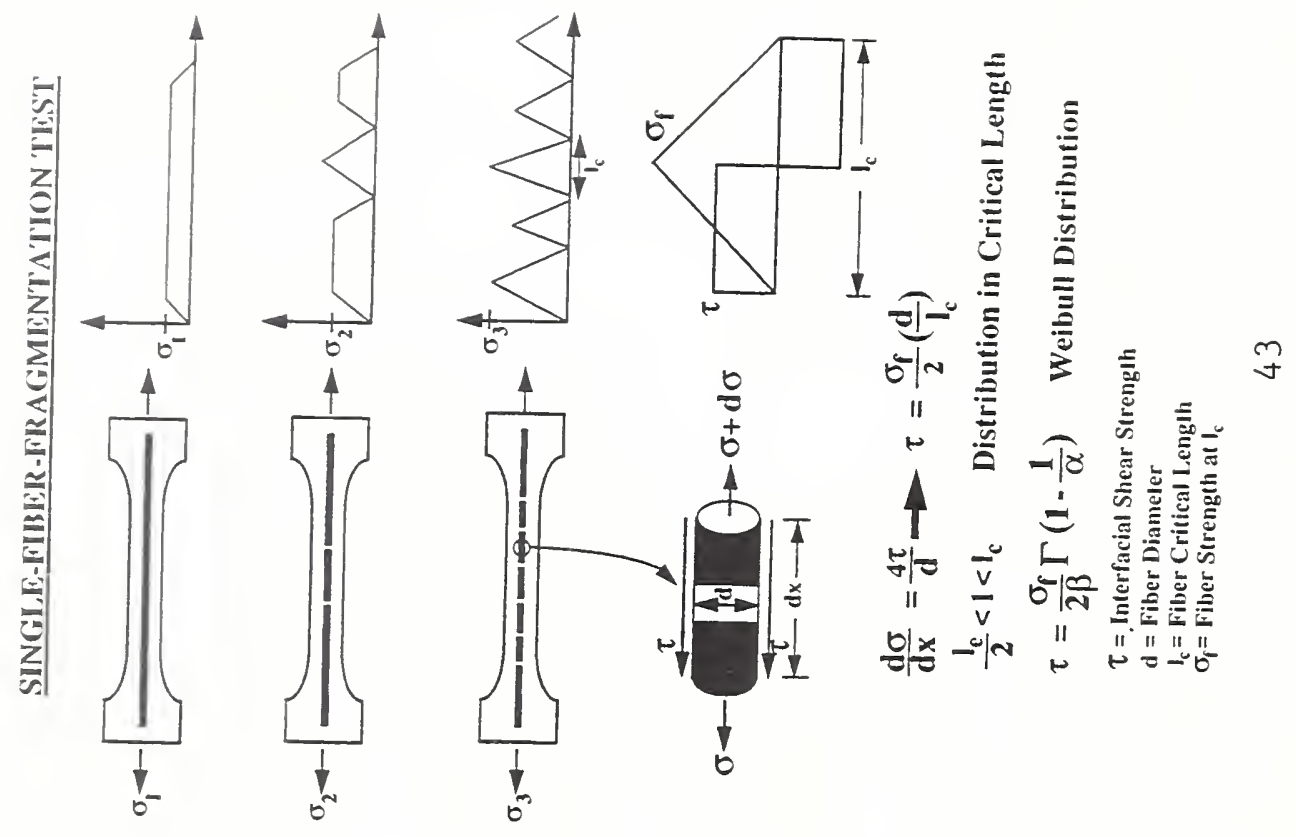

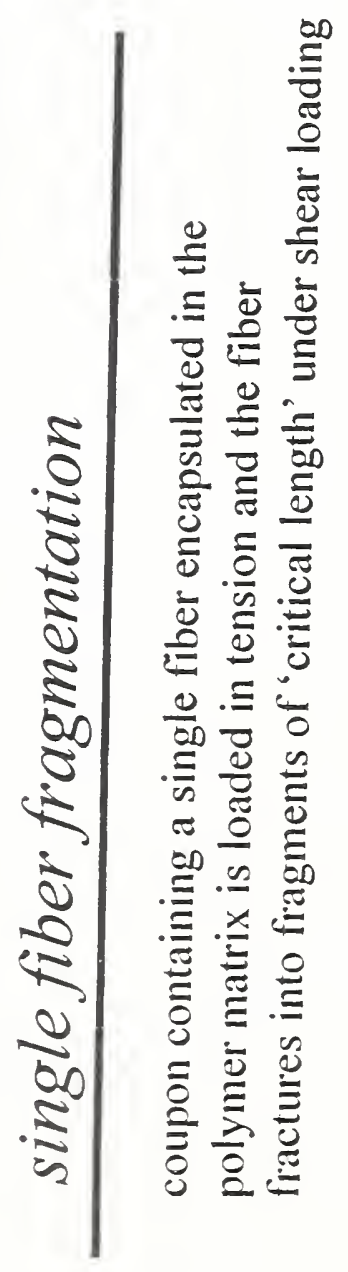



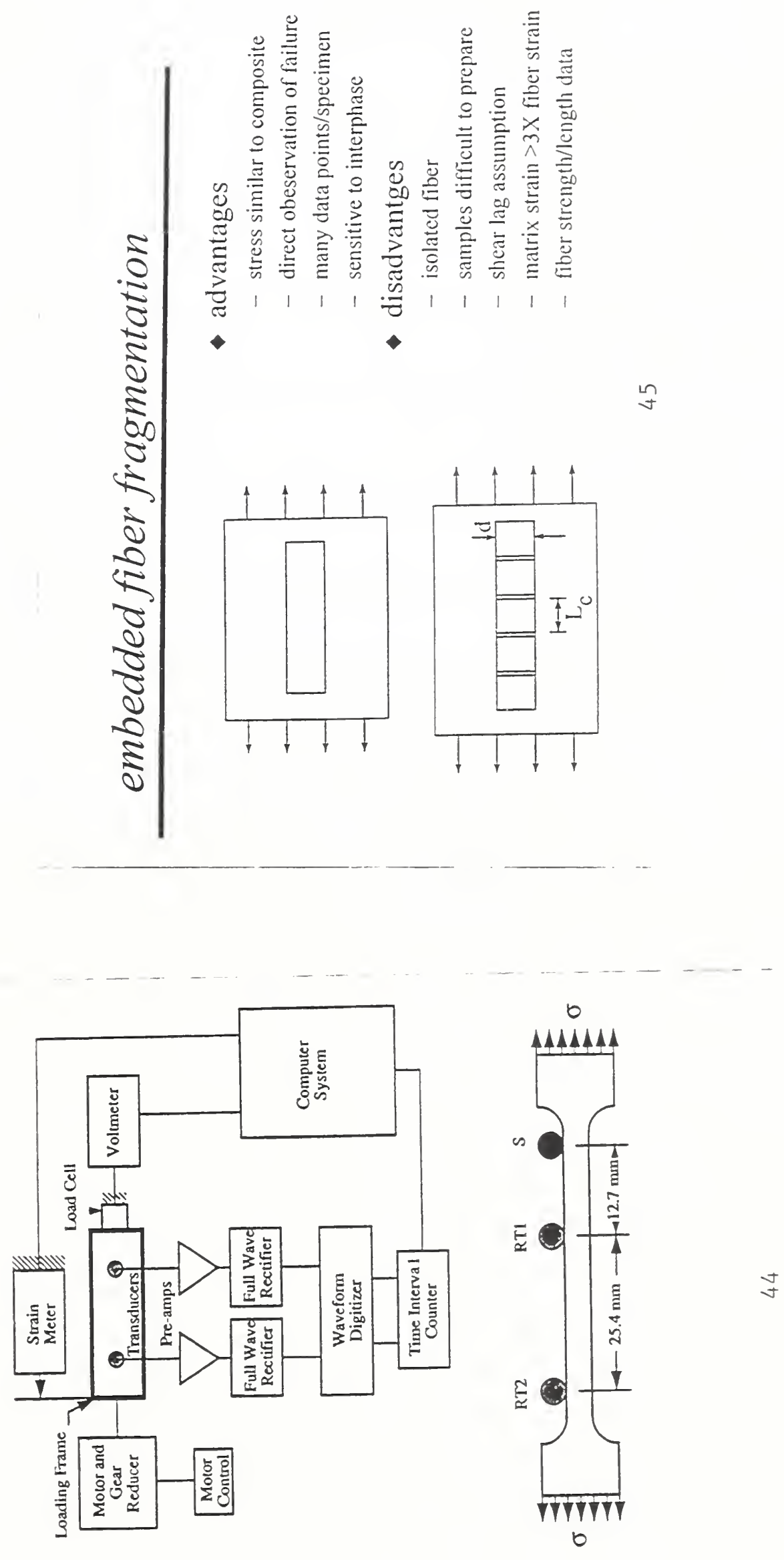

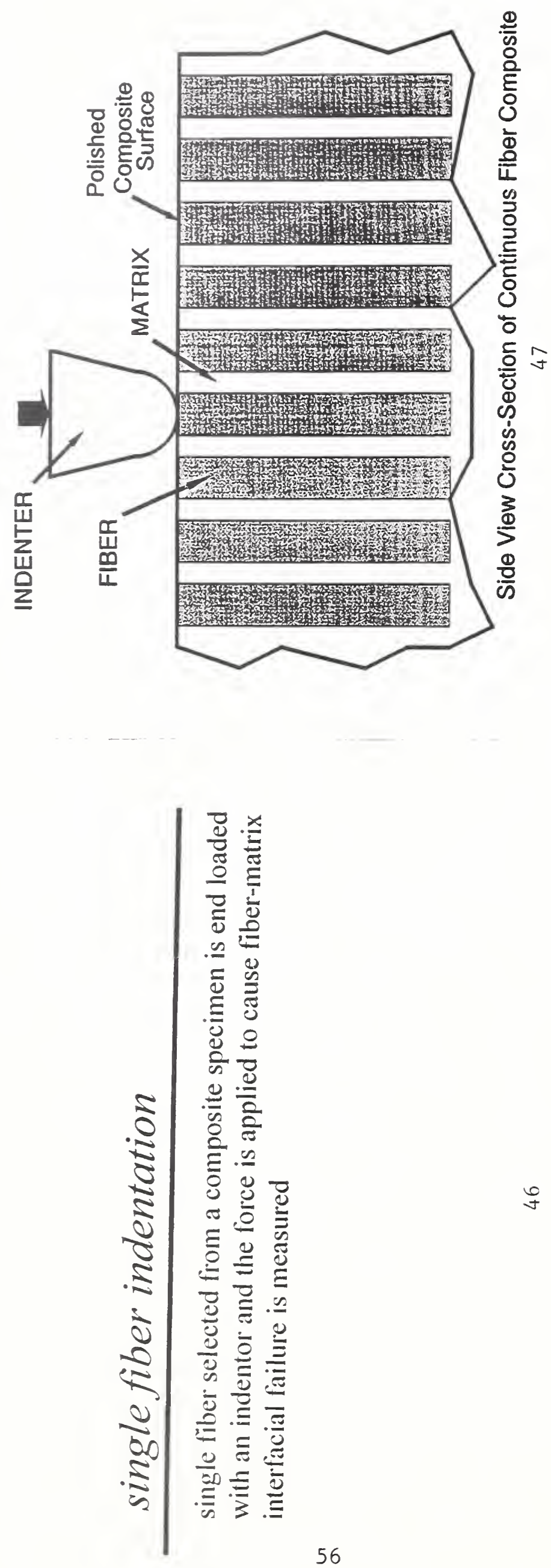

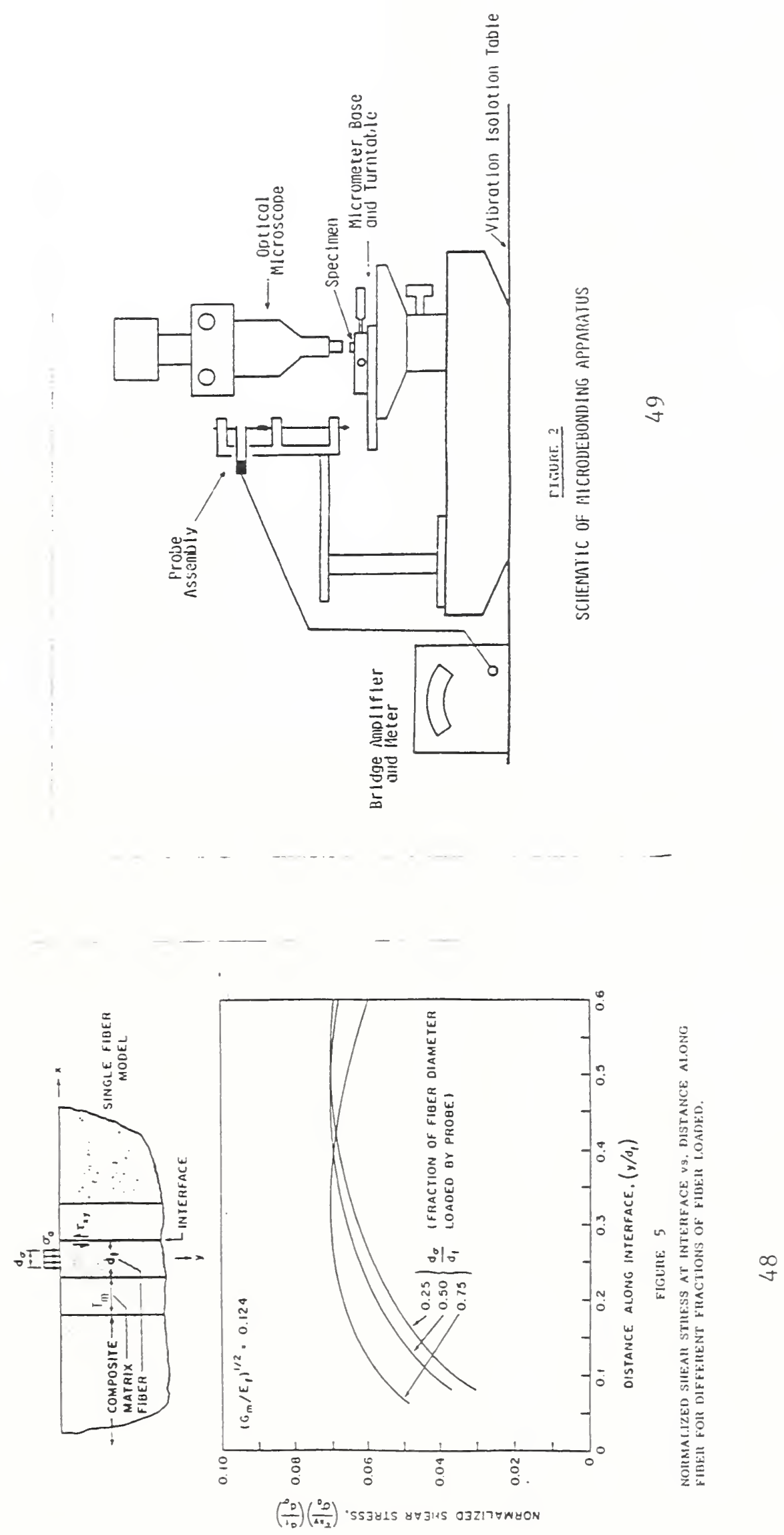

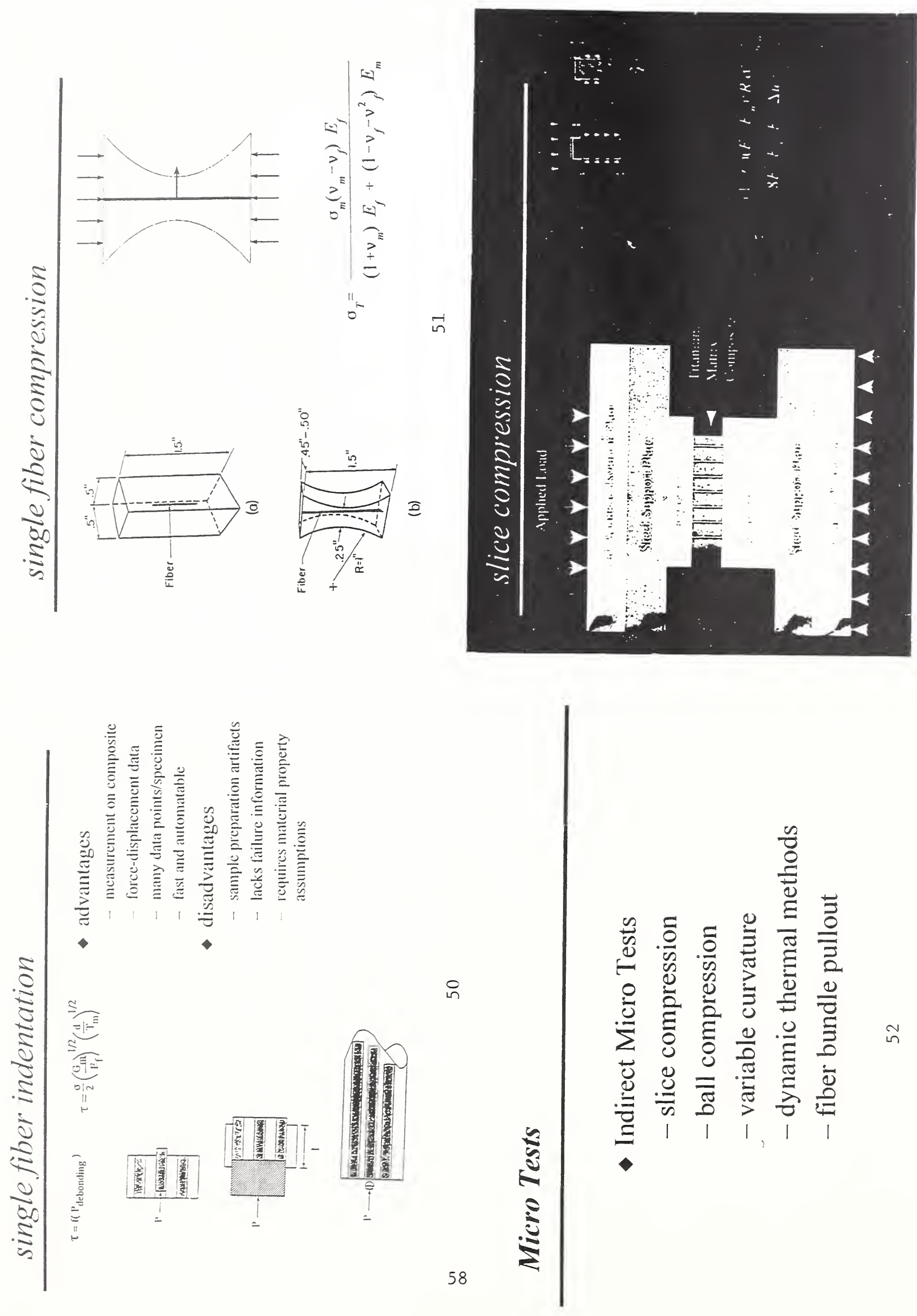

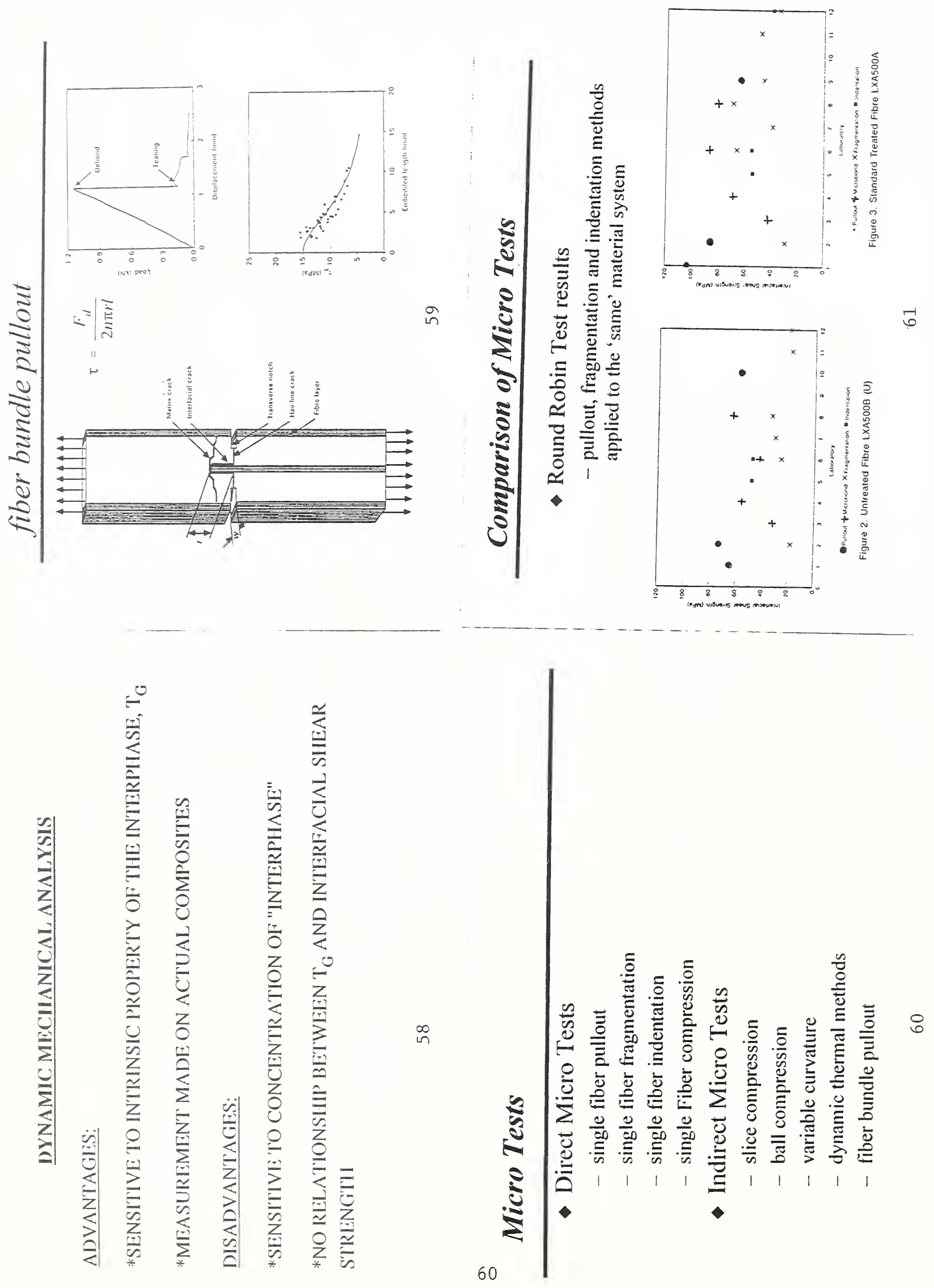

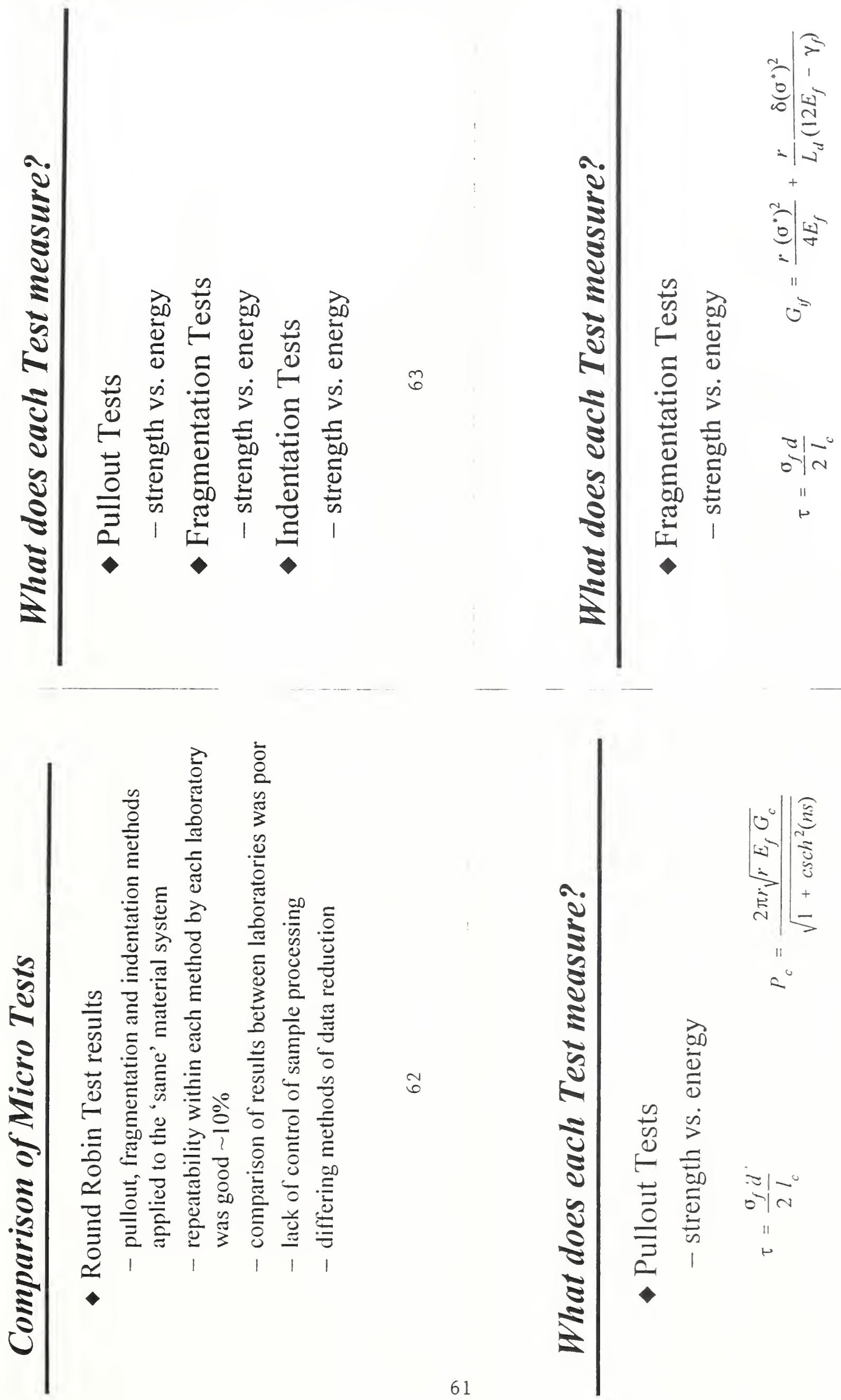

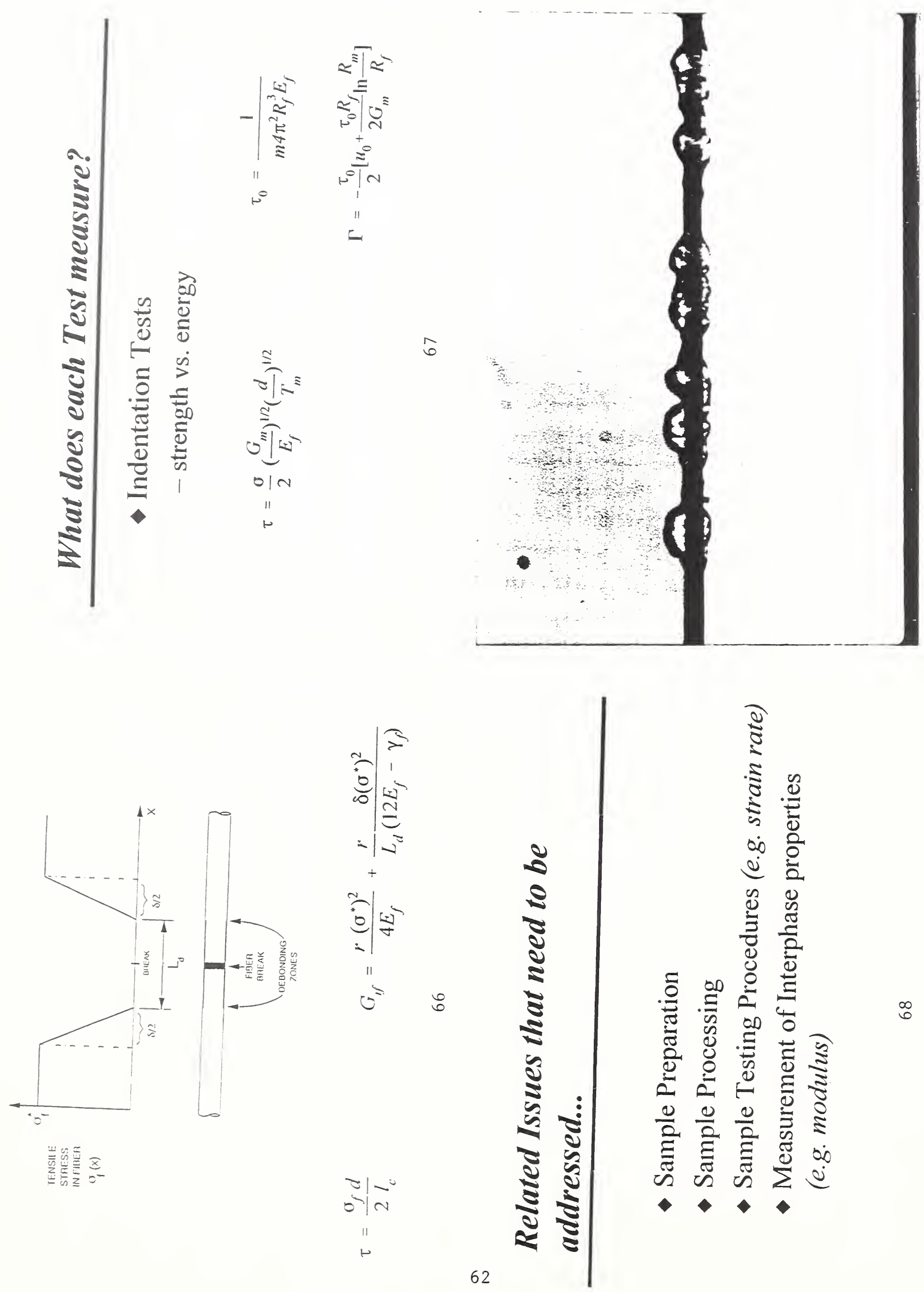

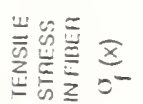

화인

$\hookleftarrow$ 

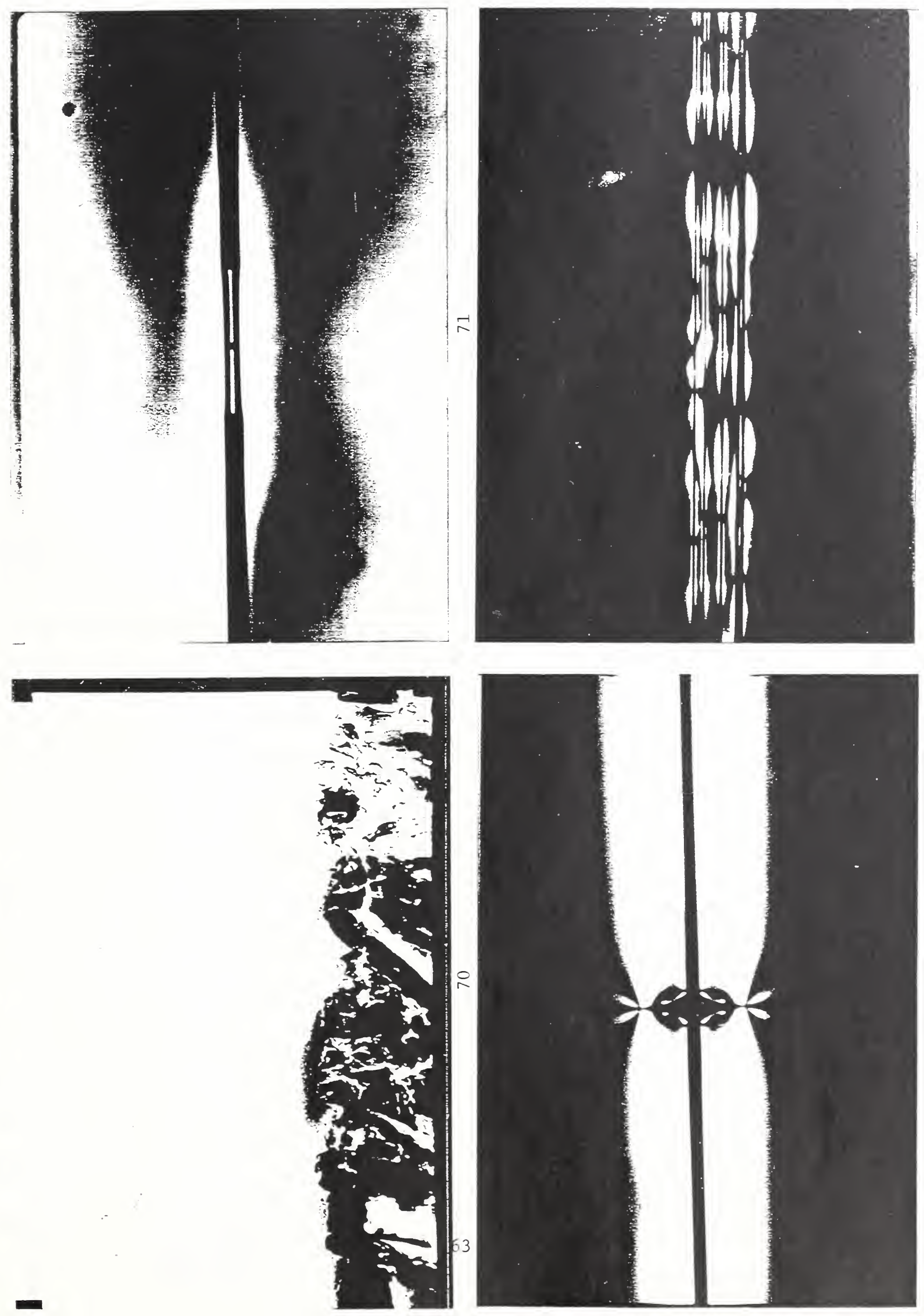


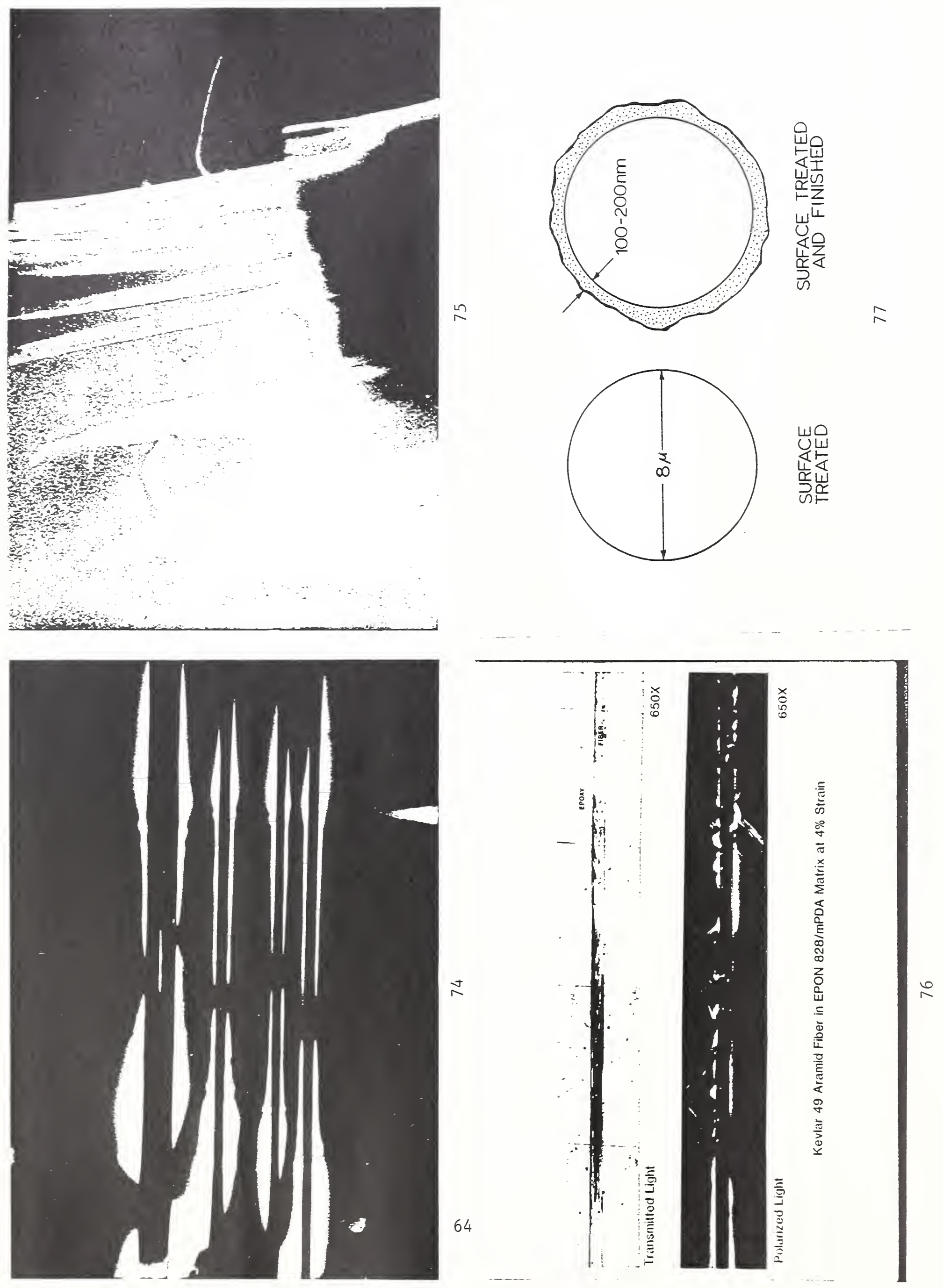



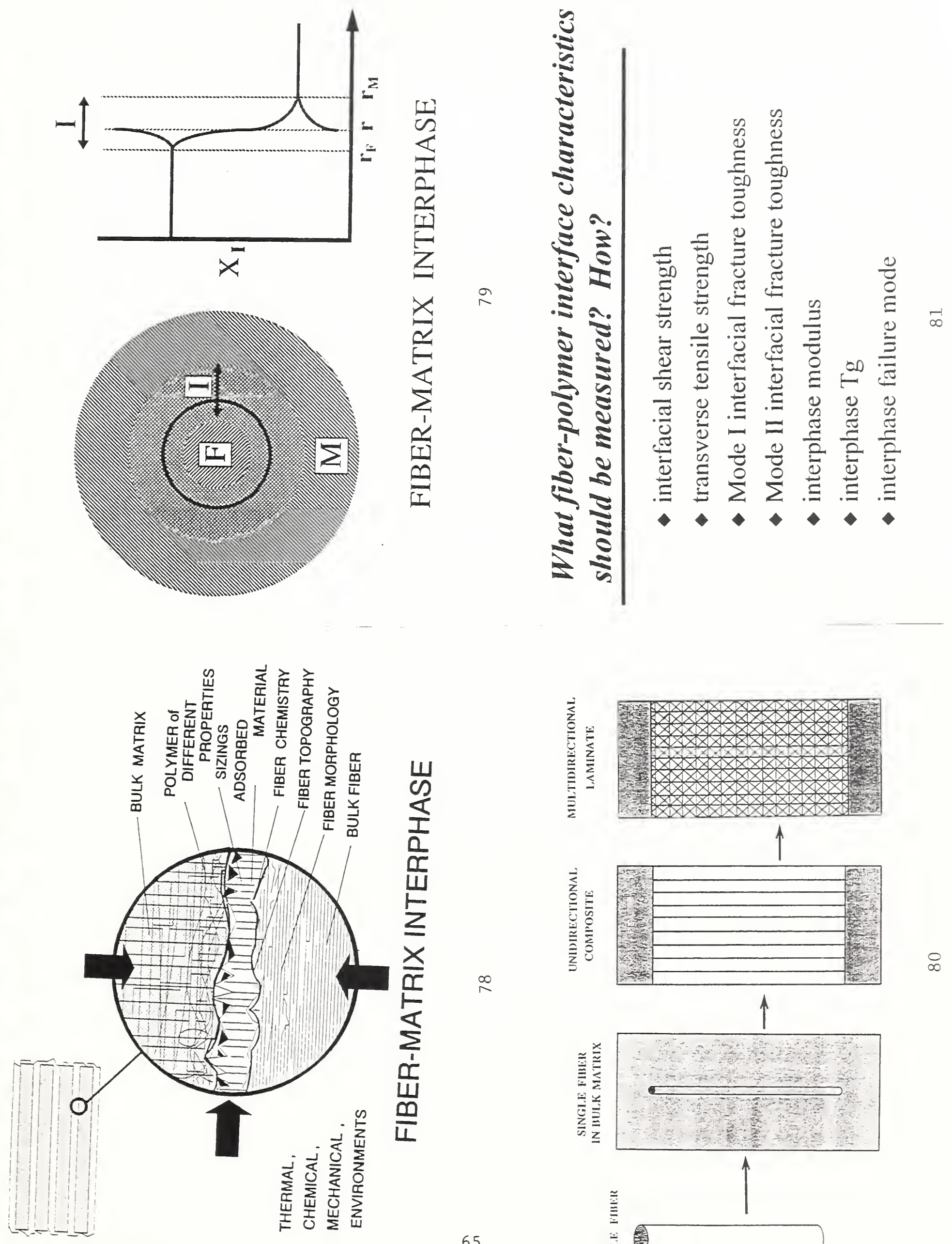

$\stackrel{\infty}{N}$

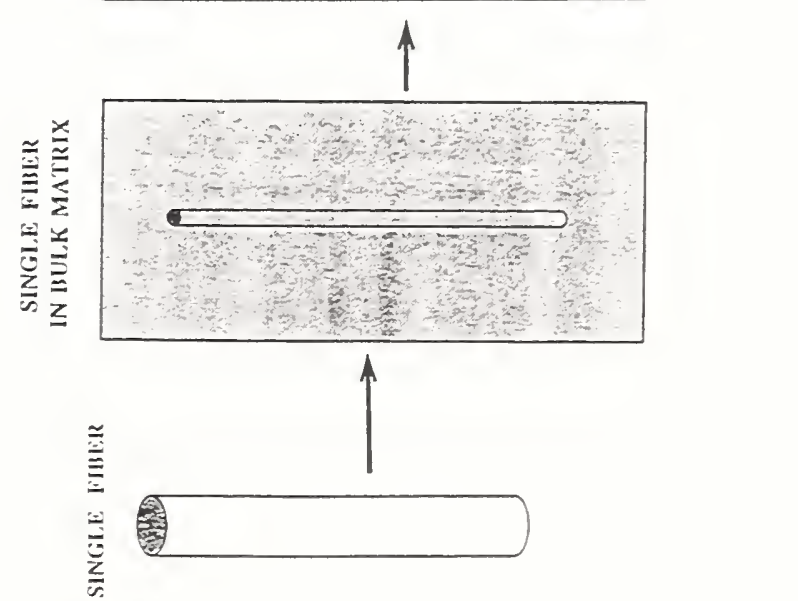





\section{THE MICROBOND SHEAR STRENGTH PULLOUT TEST}

\section{Bernard Miller}

TRI/Princeton

A useful estimate of the shear strength of a fiber/resin interface can be obtained by measuring the force needed to pull a single fiber axially out of the solid resin matrix. Since the debonding force will be mainly proportional to the length of embedded fiber, if the fiber diameter is small ( $\sim 10$ microns), the embedded length must also be minimal so that the fiber does not rupture before debonding occurs. Typically, embedment lengths must not exceed about $1.0 \mathrm{~mm}$ in order to achieve reliable shear strength testing.

The above limited immersion requirement cannot be achieved by simply using a shallow pool of resin. If the fiber surface is appreciably wetted by the liquid resin, there will be an elevated meniscus formed around the fiber, as illustrated in Figure 1. The height of this meniscus can be more than $1 \mathrm{~mm}$ and therefore this approach cannot be used with fine fibers. In addition, the resin coating will be thinner in the raised meniscus region and may rupture prematurely.

To avoid the above problems, an approach has been developed that uses only a very small amount of resin deposited as a droplet on the fiber[1]. When applied to the fiber, the liquid resin forms a concentric ellipsoid and the shape is retained when the resin is cured and becomes hard. The two critical dimensions, the fiber diameter and the embedded length, are obtained by digital optical micrometry of the cured specimen. An example of a mounted and cured droplet is shown in Figure 2.

\section{Experimental Procedure}

The fiber is pulled out of the microdroplet, usually at a rate of $1 \mathrm{~mm}$ per minute. To accomplish this, the droplet is gripped by a microvise made up of two parallel plates that form a slot with an adjustable width, as illustrated in Figure 3. The plates first are moved so that they are in contact with the bare fiber just above the resin drop. The microvise is then moved downward and the force generated is recorded until one of three possibilities has happened (see Figure 4). If the droplet is sheared from the fiber, the force reading will first rise and then fall abruptly at the critical shearing load. This will be followed by a constant force reading that represents the 
frictional force that is resisting the sliding of the free droplet along the fiber. If the resin is not hard enough, so that it flows or fractures under the loading, the force trace will show a gradual change such as that illustrated by the middle plot shown in Figure 4 . If the fiber ruptures before shearing, the force reading drops directly to zero. Figure 5 shows a typical result of droplet debonding.

The above represents one of the practical advantages of this technique over other methods for evaluating fiber/resin shear strength. One can clearly tell from the recorded force trace whether or not there has been shear debonding. (The identification of this debonding as either adhesive or cohesive will be addressed later in this paper.) The estimation of the interfacial shear strength $\tau$ is achieved by use of the equation

$$
\tau=\mathrm{F} / \pi \mathrm{dl}
$$

where $F$ is the debonding force, $d$ the fiber diameter and 1 the embedded length.

Another convenient aspect of the microbond test is that one can place more than one droplet on each fiber. As illustrated in Figure 6, this makes it possible to obtain more than one measurement from the same fiber specimen. It also leads to an interesting comparison of shearing by means of the two parallel plates and by a previously freed droplet. In the latter case, the force is supplied by a completely symmetrical load. When results for first and second droplet pairs sheared in this manner are compared, it seems that the parallel plates.have essentially the same effect as the solid droplet shearing[2].

Thermosetting resin droplets can be applied to a fiber as a liquid, as previously described. Thermoplastic resins also can be used for such bond strength testing. For such materials, a thin strip of the resin in film form is folded and hung on the horizontally mounted fiber. The combination is then heated to a temperature that is high enough to cause the resin to melt and flow. When this happens most of the resin will fall away, leaving a small amount that will form a droplet around the fiber[3]. This process is illustrated in Figure 7.

Nearly every fiber/resin combination will show a relatively wide, but symmetrical, distribution of bond strengths, if enough specimens are tested $(>20)$. A major cause of this is believed to be fiber 
surface nonuniformity. Since each microbond experiment is performed on a very small portion of the fiber specimen, it can be expected that very few droplets will encounter exactly the same fiber surface conditions. Supporting evidence for this has been obtained by comparing the results of replicate data for near-neighbor pairs (no more than a few millimeters apart) with pairs selected randomly from a complete data pool. The former showed noticeably smaller differences as compared to the latter[4].

Other sources of error have been considered and discussed. If the drop volume is reduced, the "contact angle" between the drop and the fiber will become smaller. (Contrary to the usual teachings, there is no singular characteristic contact angle for a liquid/solid combination under these conditions.) When this angle is small, the force applied by the shearing plate will be partially dissipated for shearing purposes and will supply a compression force that can augment the fiber/resin bonding[5]. This will result in an apparent bond strength that is higher than it really is.

Whatever the orientation of the surface of the drop, it is also necessary that the shearing surfaces be as close as possible to the fiber surface[6]. This "gap width effect" is illustrated by the results shown in Figure 8.

\section{Examples of Applications}

\section{The Effect of Hydrothermal Exposure}

Bonded droplets were divided into three subgroups. One group was tested as controls to establish the original shear strength of each fiber/resin combination studied. A second group was exposed to hot water $\left(88^{\circ} \mathrm{C}\right)$ for 24 hours and then tested. The third group was exposed to the same hot water treatment and then dried before bond strength testing. The results of this study[7] are shown in Figure 9. The Kevlar/fiber pairs both showed about a 20\% loss of bond strength after hot water treatment, which was completely reversible upon drying. In contrast, the glass/epoxy bond was more drastically weakened and could not be completely regenerated by drying.

\section{Regeneration of Sheared Fiber/Resin Bonds}

Thermoplastic polycarbonate droplets on Kevlar fibers were debonded (and their bond strengths measured) and then left on the fibers so that they could be re-bonded. A matrix of 
time/temperature combinations was used. The results are given in Table I, where it can be seen that complete regeneration of the original bond strength was achieved if the time and temperature combination was sufficient to produce adequate flow of the thermoplastic resin[8].

In contrast, sheared Kevlar/epoxy combinations recovered only about $50 \%$ of their original bond strength when reheated to the same temperature as that when they were originally cured (see Figure 10).

\section{Studies of Bond Rupture Mechanisms}

The exact location of the bond on the fiber can be established with considerable accuracy by means of wettability scanning, using an instrument such as the TRI wetting force analyzer shown in Figure 11. The fiber is scanned from its free end and the wettability trace clearly identifies the original position of the droplet (Figure 12). The droplet is then sheared and the wetting scan repeated. This second scan will often reveal one of the following types of bond rupture[9]:

1. Cohesive failure in the fiber surface layer ... In this case, as shown in Figure 13, the wettability of the stripped layer of the fiber can be different from that of the original outer surface, indicating that the debonding took place in the fiber itself.

2. Adhesive failure between fiber and resin... The debonding process has left the fiber surface as it was before bonding, as shown by the unchanged wettability (Figure 14).

3. Cohesive failure in the resin ... This will leave a residual layer of resin, either uniform or irregular, which can be identified by the increase in the wetting force response because the perimeter of the solid has been increased. This type of scanning can identify very small changes in perimeter, as illustrated in Figure 15.

$$
\text { * } \quad \text { * } \quad * \quad * \quad *
$$

These examples of the use and application of the microbond shear strength measurement technique should suffice to illustrate that it can be an effective tool for dealing with some of the problems and questions that are associated with fiber/resin composite materials. 


\section{References}

1. B. Miller, P. Muri \& L. Rebenfeld, Comp. Sci. Tech., 28, 17 (1987)

2. U. Gaur, \& B. Miller, Comp. Sci. Tech., 34, 35 (1989)

3. U. Gaur, G. Desio \& B. Miller, Plastics Eng., 45, 43 (Oct. 1989)

4. U. Gaur \& B. Miller "Controlled Interphases in Composite Materials", H. Ishida, Ed., Elsevier, NY pp. 723-730 (1990)

5. B. Miller, U. Gaur, \& D.E. Hirt, Comp. Sci. Tech., 42, 207 (1991)

6. C.T. Chou, U. Gaur \& B. Miller, Comp. Sci. Tech., 51, 111 (1994)

7. U. Gaur, C.T. Chou \& B. Miller Composites, 25, 609 (1994)

8. C.T. Chou, U. Gaur \& B. Miller, J. of Adhesion (in press)

9. C.T. Chou, U. Gaur \& B. Miller, Comp. Sci. Tech., 48, 307 (1993) 
Table I. Regenerated IFSS (MPa) as a Function of Reprocessing Conditions Kevlar 49/PC (Original IFSS $=34.8 \mathrm{MPa}$ )

\begin{tabular}{llllll} 
Time $\downarrow / T e m p \rightarrow$ & $275^{\circ} \mathrm{C}$ & $250^{\circ} \mathrm{C}$ & $225^{\circ} \mathrm{C}$ & $200^{\circ} \mathrm{C}$ & $180^{\circ} \mathrm{C}$ \\
\hline 5 minutes & 31.8 & 28.5 & $\sim 0$ & $\sim 0$ & $\sim 0$ \\
20 minutes & -- & 35.0 & 15.6 & 11.7 & $\sim 0$ \\
30 minutes & 36.5 & 38.2 & 16.7 & 19.9 & 15.6 \\
1 hour & 37.9 & 36.0 & 25.0 & 23.2 & 17.1 \\
2 hours & 33.0 & 38.1 & 29.9 & 23.5 & - \\
3 hours & --- & -- & 32.0 & -- & 18.3 \\
6 hours & --- & -- & --- & 26.2 & 10.8 \\
1 day & -- & - & 27.0 & 28.5 & 15.9 \\
3 days & -- & - & 32.5 & 34.8 & 20.4 \\
1 week & - & - & - & - & 24.0 \\
\hline
\end{tabular}

Note: 1. At least 15 specimens were tested at each condition,

2. Typical $95 \%$ confidence level ranges from $5 \%$ to $10 \%$ of the average value. 


\section{Figure Captions}

Figure 1: Extended embedded length produced by a meniscus and potential rupture of the meniscus before debonding.

Figure 2: Resin droplet on a fiber before debonding.

Figure 3: Arrangement for shear debonding (top) and enlarged schematic of a resin droplet on a fiber under the shearing plates (bottom).

Figure 4: Force traces for the three possible results of a shear test.

Figure 5: Appearance of sheared droplet after debonding from an E-glass fiber. Arrow represents direction of shearing force.

Figure 6: Arrangement for measuring shearing force for sequential debonding of two microdroplets on a single fiber. Debonded droplet is used to shear another droplet.

Figure 7: (a) Schematic illustrating cutting of "V" strip. (b) Melting of "V" strip over single fiber to form microdroplets.

Figure 8: Effect of gap width on microbond measurements (Kevlar 49/Epon 828).

Figure 9: Regeneration of microcomposite bond strength upon drying at $115^{\circ} \mathrm{C}$.

Figure 10: Shear strength of Kevlar 49/epoxy microdroplet assemblies after multiple shearing/heating cycles.

Figure 11: Wetting force measurement apparatus.

Figure 12: Advancing wetting force scan providing exact location of microdroplets.

Figure 13: Cohesive failure in fiber.

Figure 14: Fiber/Resin adhesive failure.

Figure 15: Cohesive failure in resin. 


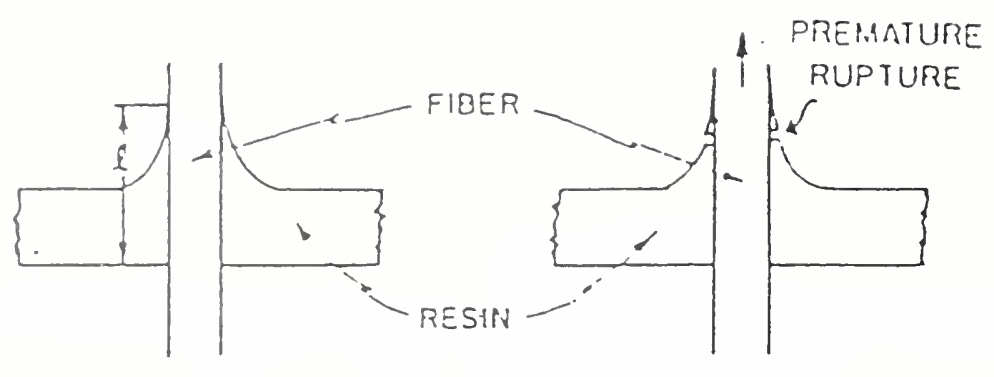

Figure 1: Extended embedded length produced by a meniscus and potential rupture of the meniscus before debonding.

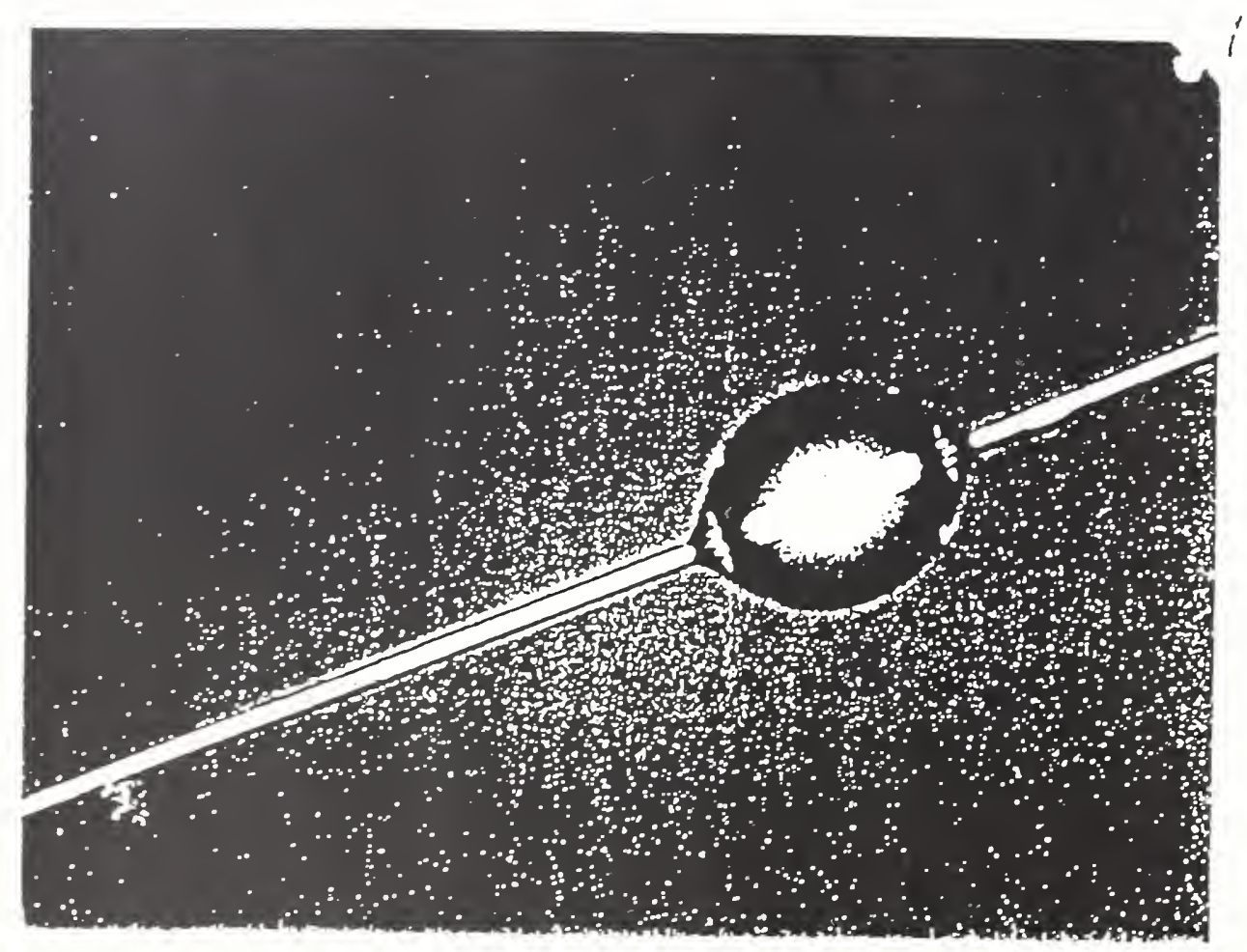

Figure 2: Resindeplet on a fiber before debonding. 

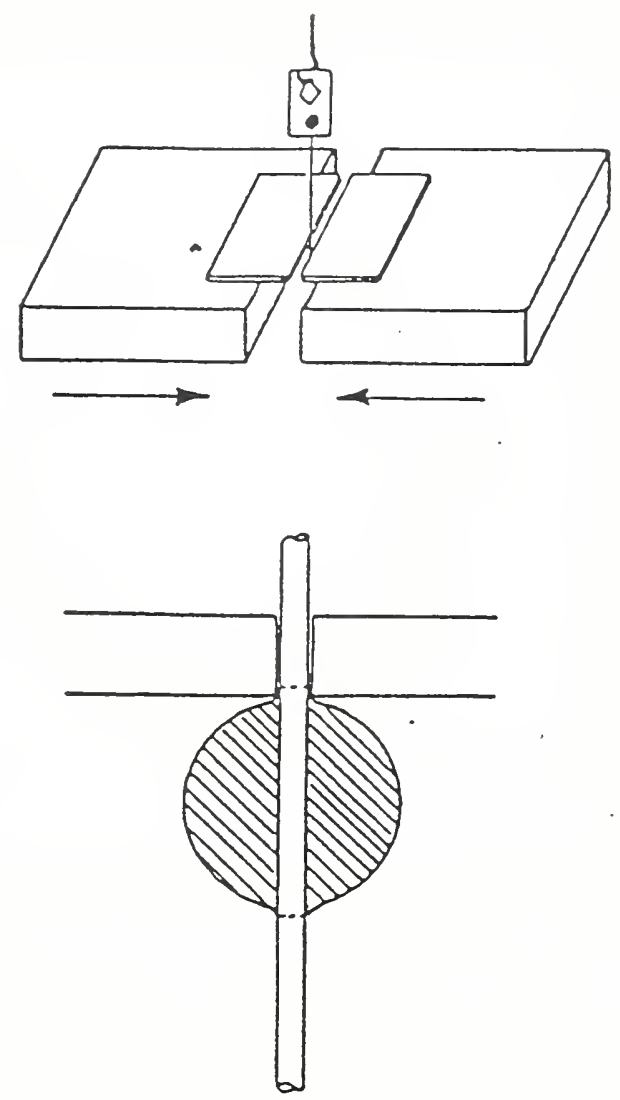

Figure 3: Arrangement for shear debonding (top) and enlarged schematic of a resin droplet on a fiber under the shearing plates (bottom).

E

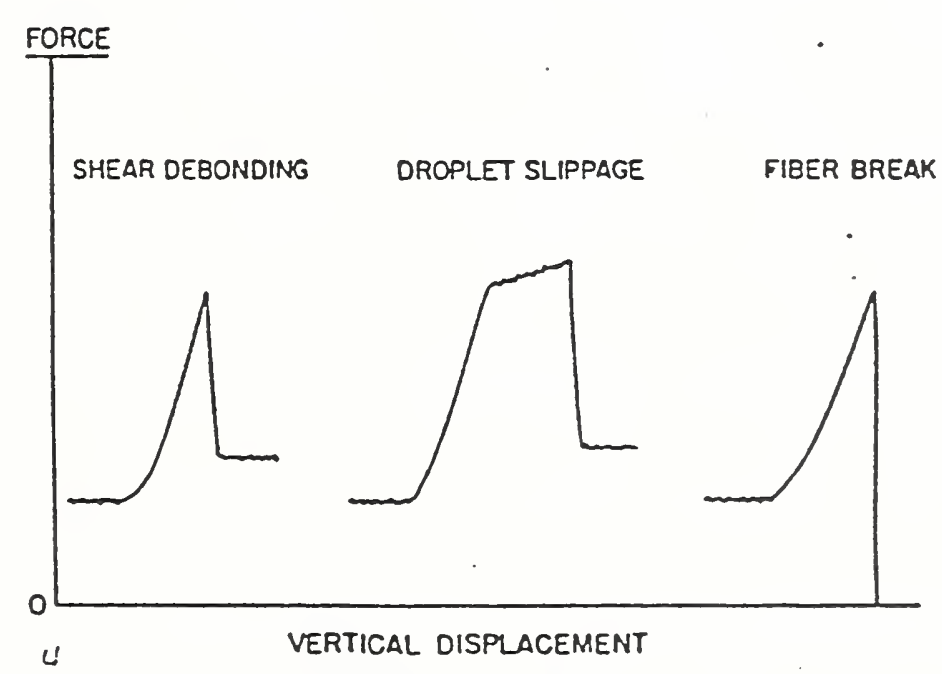

Figure 4: Force traces for the three possible results of a shear test. 


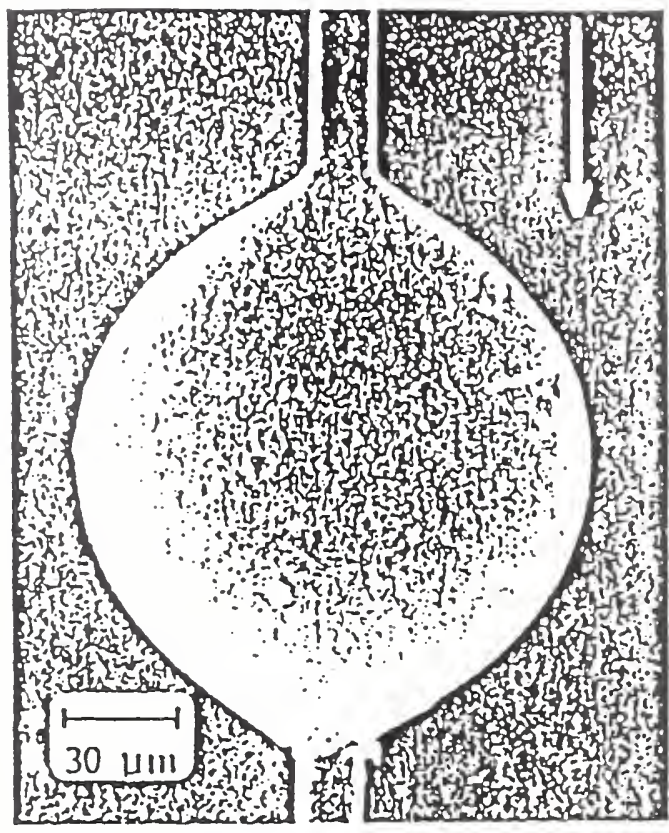

Figure 5: Appearance of sheared droplet after debonding from an E-glass fiber. Arrow represents direction of shearing force.
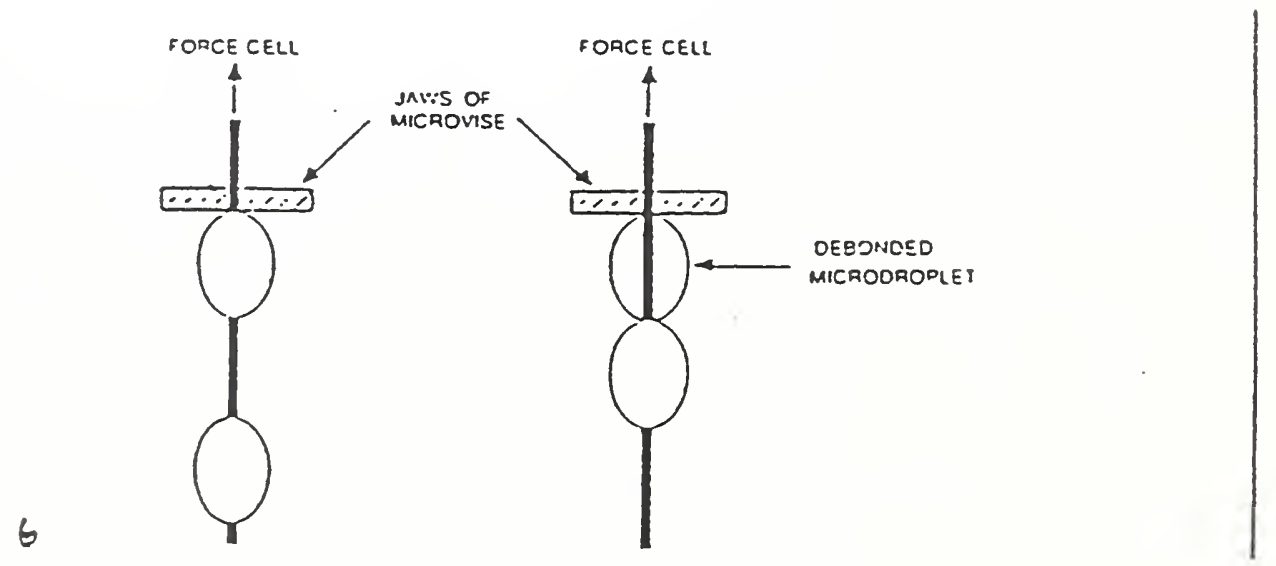

Figure 6: Arrangement for measuring shearing force for sequential debonding of two microdroplets on a single fiber. Debonded droplet is used to shear another droplet. 

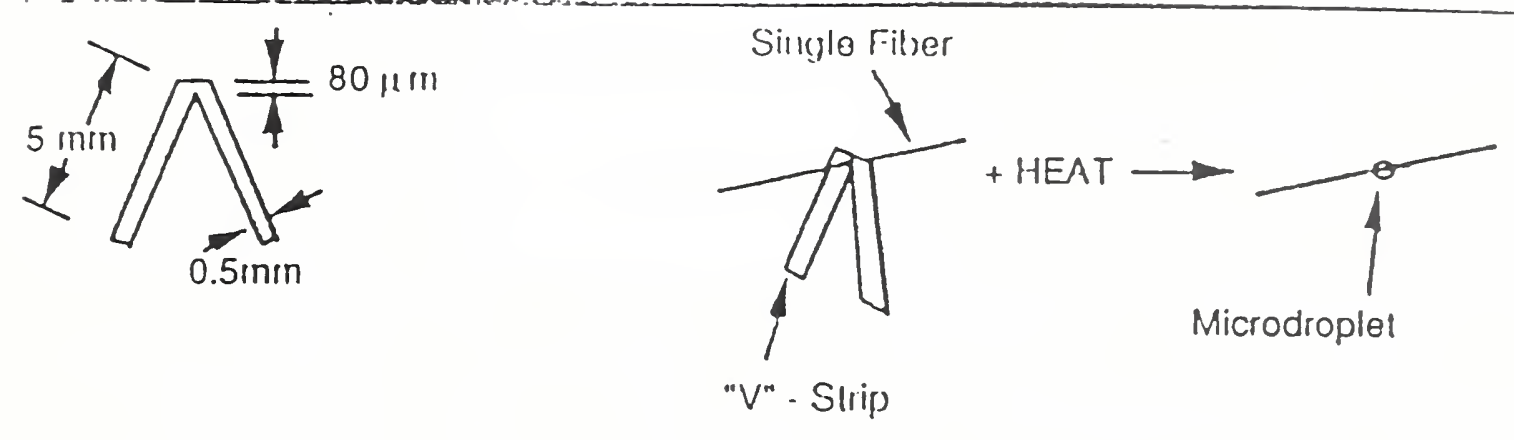

Figure 7: (a) Schematic illustrating cutting of "V" strip. (b) Melting of "V" strip over single fiber to form microdroplets.

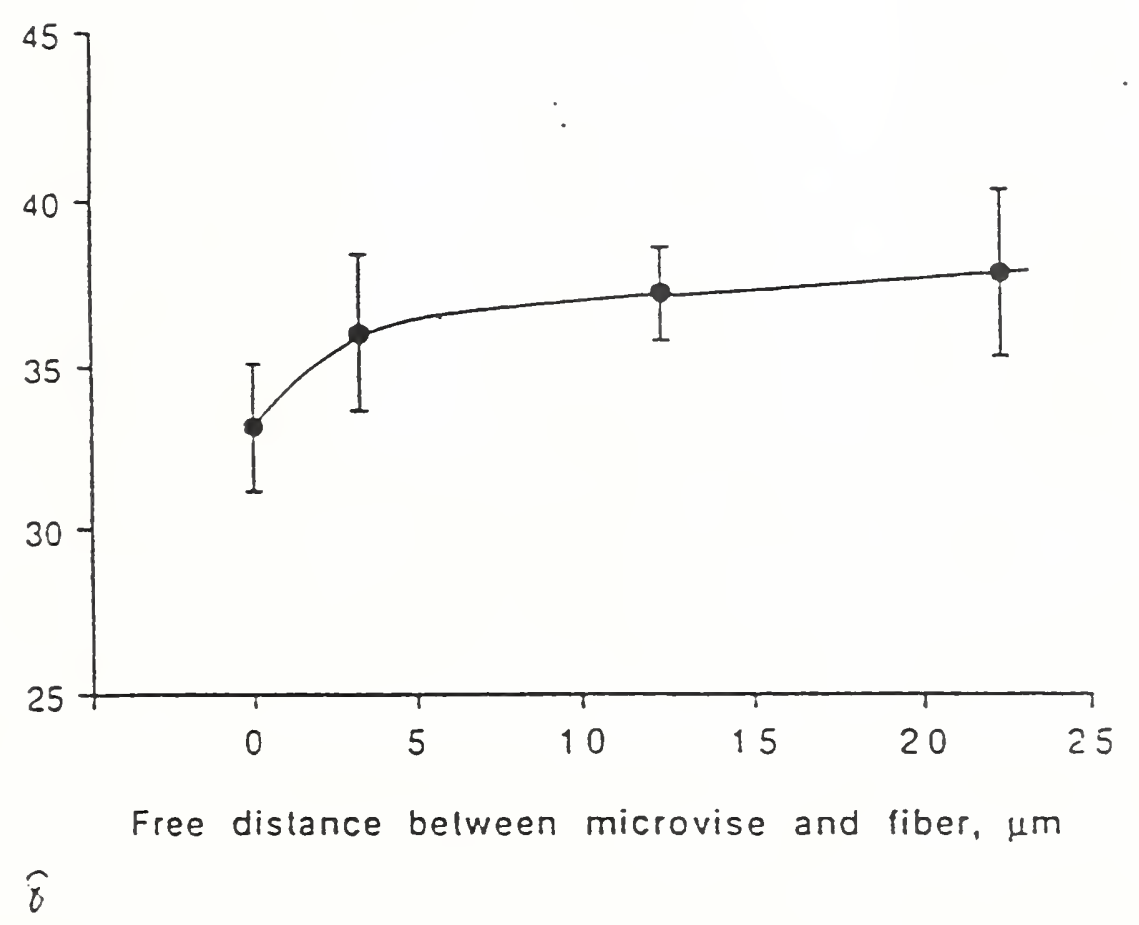

Figure 8: Effect of gap width on microbond measurements (Kevlar 49/Epon 828). 
Residual Bond Strength, \%

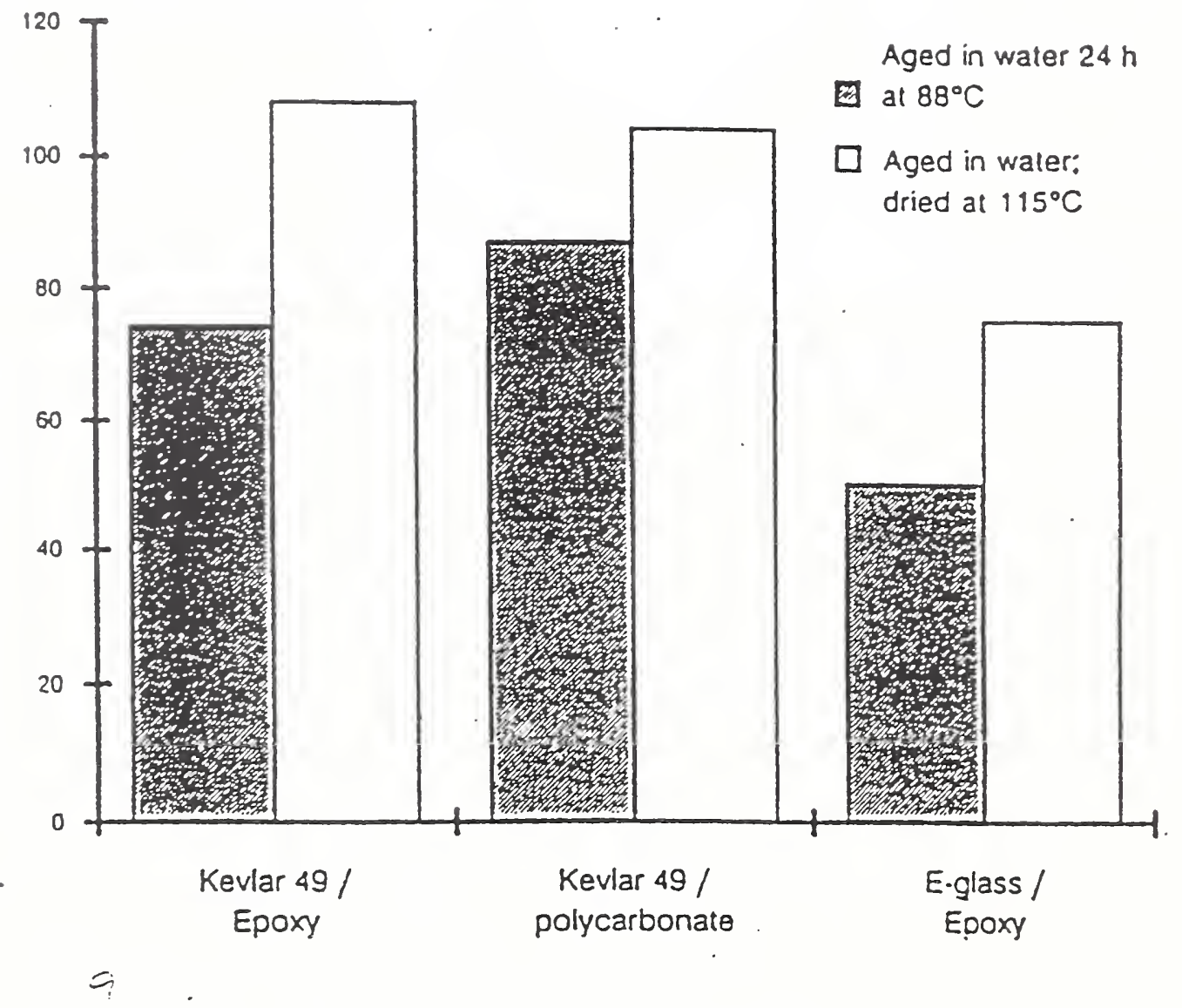

Figure 9: Regeneration of microcomposite bond strength upon drying at $115^{\circ} \mathrm{C}$. 


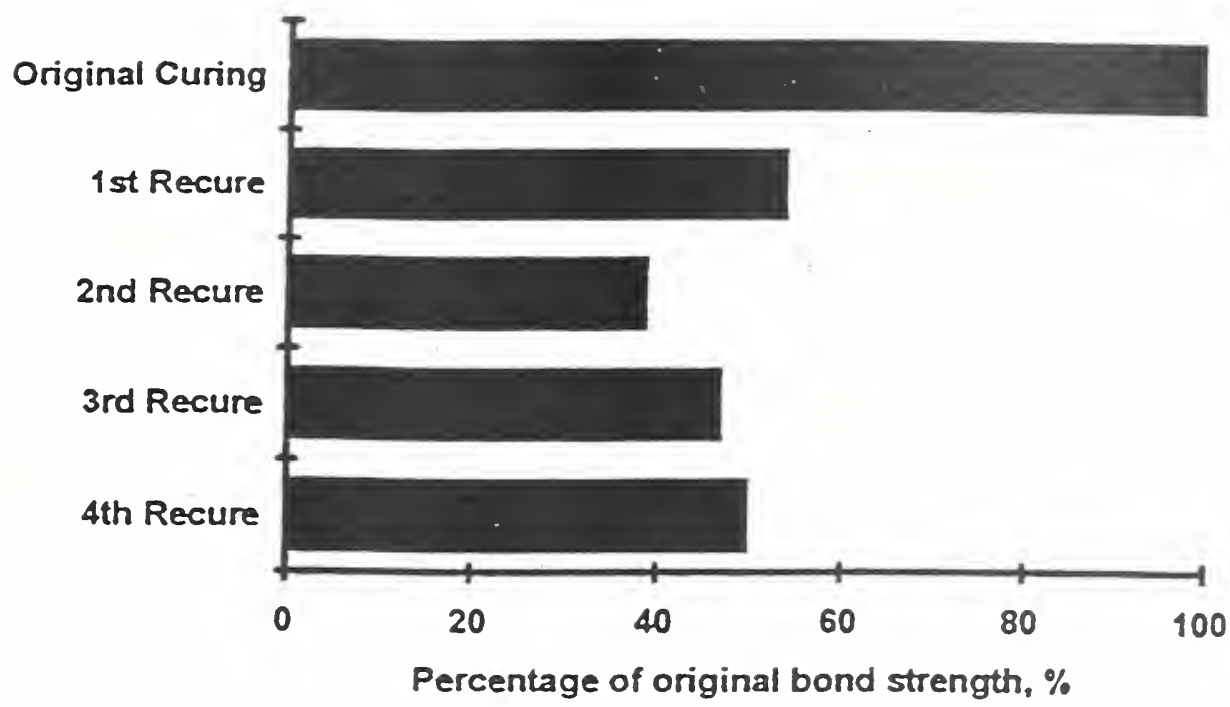

Figure 10: Shear strength of Kevlar 49/epoxy microdroplet assemblies after multiple shearing/heating cycles. 


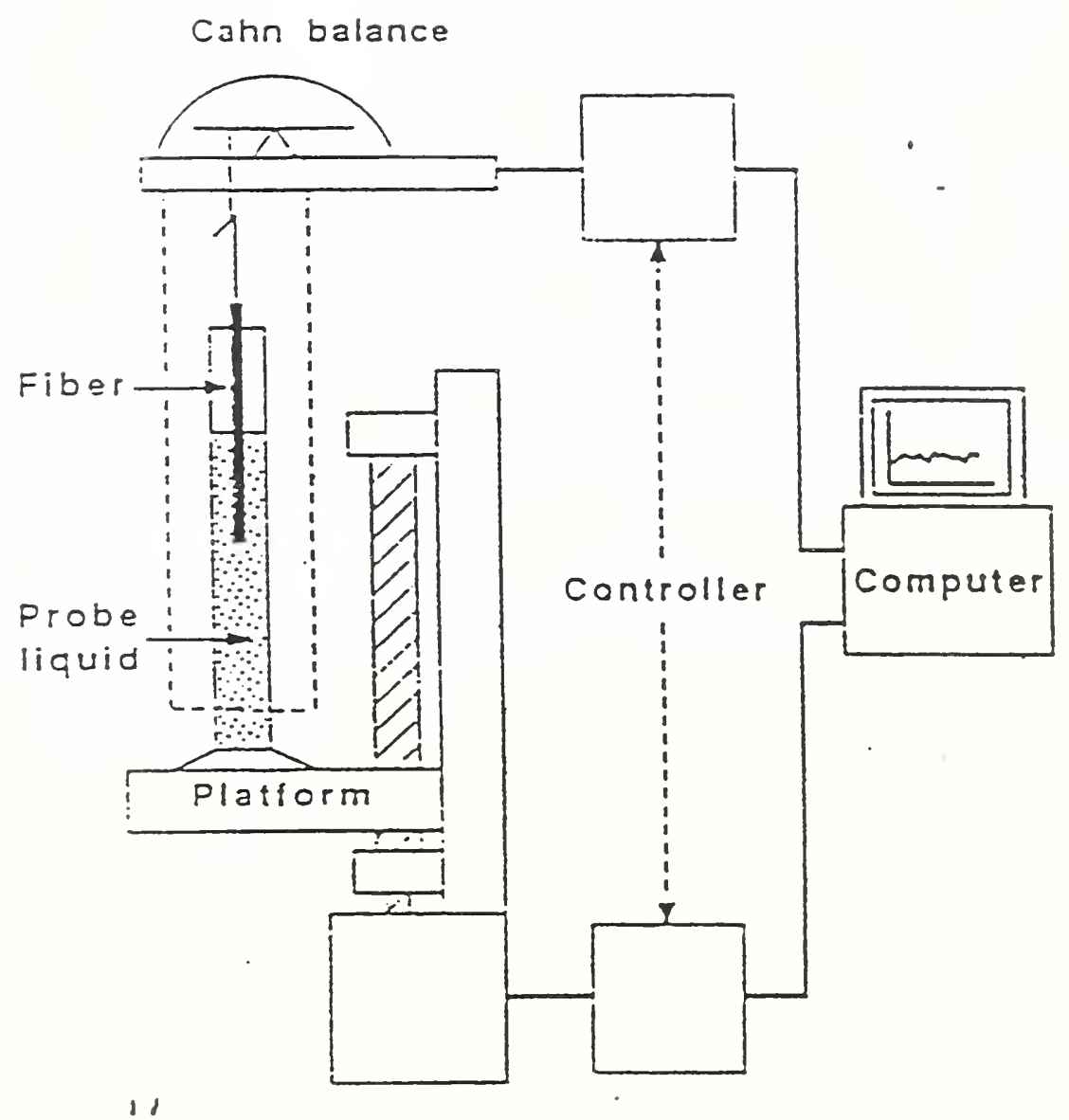

Figure 11: Wetting force measurement apparatus. 


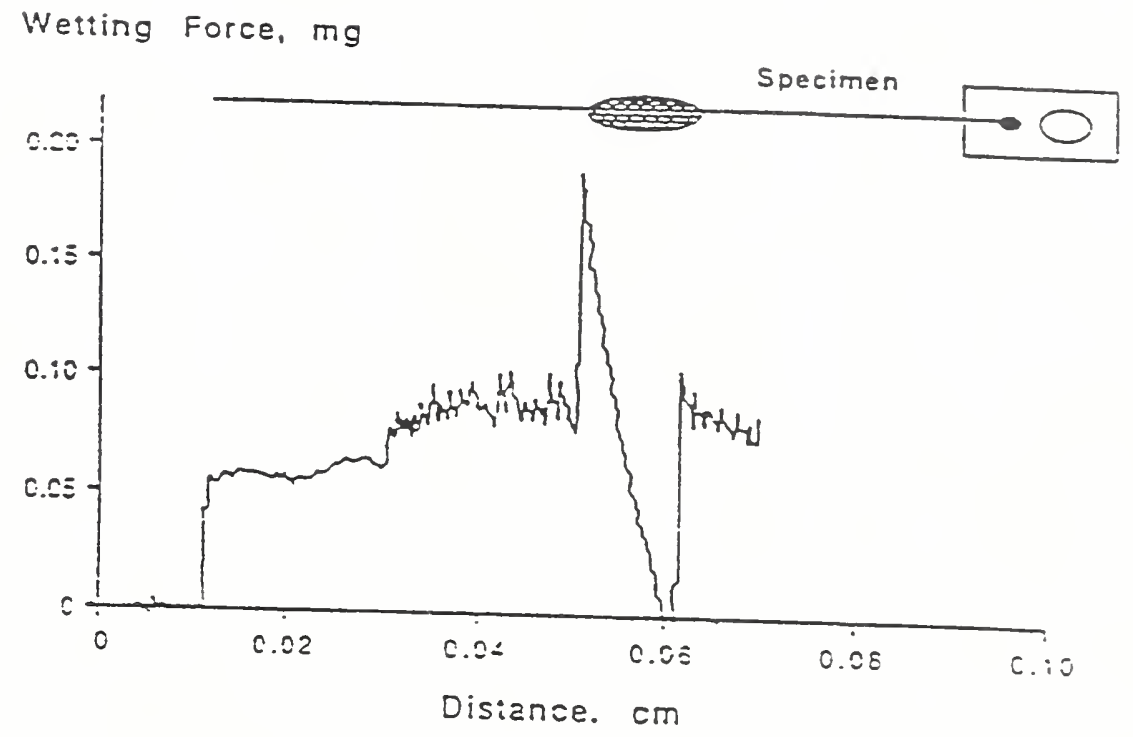

Figure 12: Advancing wetting force scan providing exact location of microdrople 

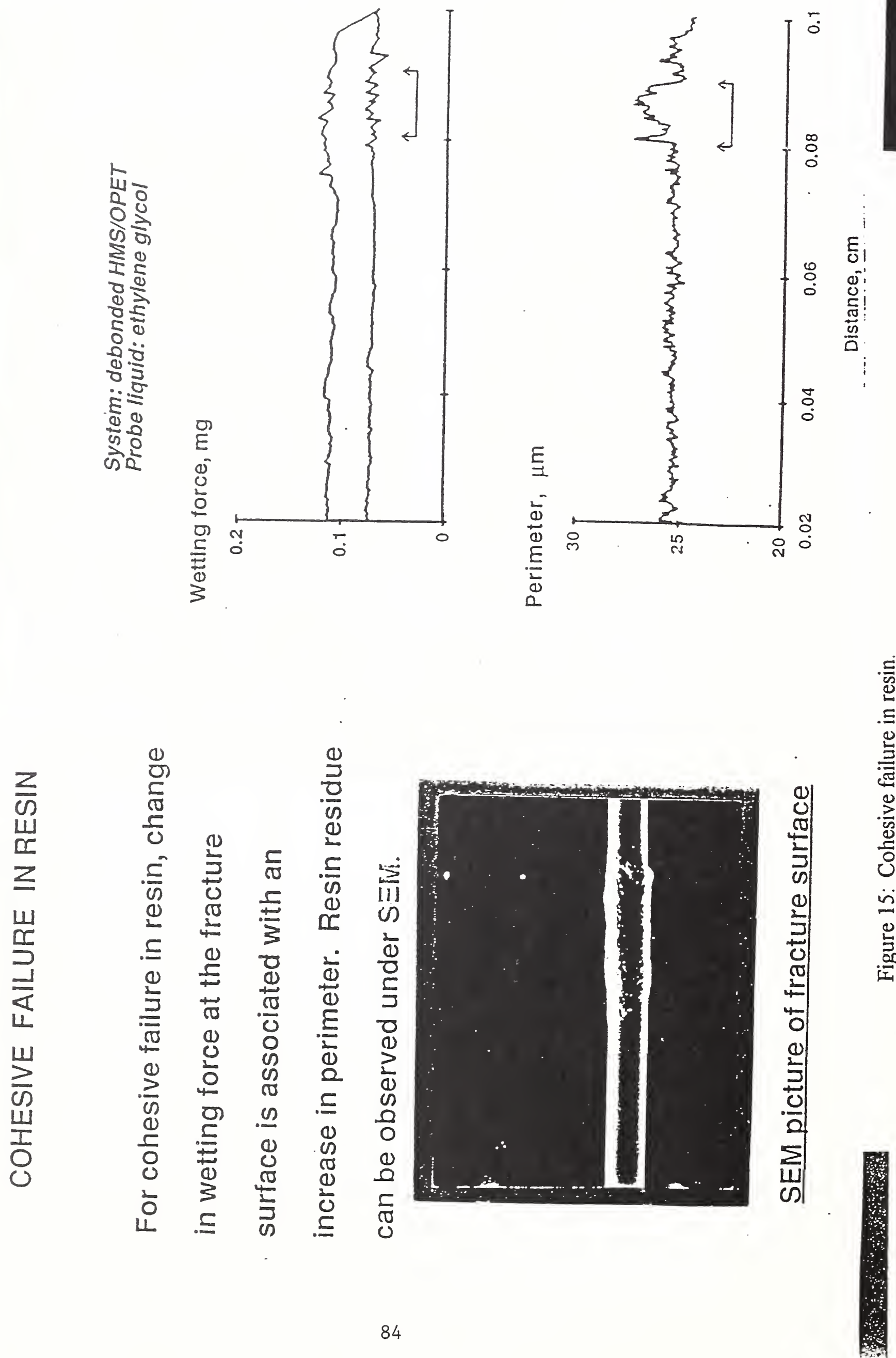
NIST Workshop Presentation

Statistical Analysis of Data from the Single Filament Composite Test and Related Micromechanical Issues

S. Leigh Phoenix

Department of Theoretical and Applied Mechanics

Cornell University

Ithaca, NY 14853

The single-filament-composite (SFC) test has seen widespread use over the past 20 years as a means of evaluating the interfacial shear strength between the fiber and matrix, often giving results in terms of a single parameter, $\tau$. As the original theory was conceived, if one envisions the straining of a single filament in a perfectly plastic matrix whereby the fiber breaks up into many fragments, the idea of a single $\tau$ makes sense. But polymer matrices and their interactions with the fiber at the interface are much more complicated, so that more sophisticated micromechanical models and representations have been pursued. The simplest extension involves shear-lag models with two $\tau$ values over different portions of the fibers near breaks (e.g. Henstenburg and Phoenix, 1989; Hui Phoenix and Kogan, 1996) which reflect matrix perfect plasticity up to debonding followed by interfacial slip and associated frictional shear tractions. More complicated elastic-plastic-debond models require the introduction of strain and displacement limiting concepts to determine when debonding is initiated and include an elastic zone beyond the yield zones (Gulino and Phoenix, 1991; Beyerlein and Phoenix, 1997). Still more complicated models add in aspects of full elasticity solutions in the presence of thermal and curing shrinkage as well as Poisson effects that affect the frictional shear tractions by varying the normal tractions along the fiber surface (Nairn and Liu, 1996) Other models approach the problem in quite a different way using energy balance ideas which largely 
bypass local interfacial stress characterization in favor such concepts as debonding energy, interface energy and energy release rate.

With all these levels of sophistication being pursued for the micromechanics, there is the issue of actually measuring the quantities being proposed, a difficult task given the size scales involved (approaching one micron). MicroRaman spectroscopy and photoelasticity techniques have proven successful in many cases and show great promise (Schadler and Galiotis, 1995), but they are very time consuming and are limited to certain geometries and material types. An indirect approach to determining interfacial stress profile information has been to monitor the fragment length distribution as a function of strain, the most common measurement being the final fragment length distribution (Henstenburg and Phoenix, 1989). In the single $\tau$ case (or assuming it is useful as an average of some sort), by knowing the fiber strength at the scale of the fragments, estimates of $\tau$ can be attempted using simple force balance formulas on final fragments, as have been proposed. As we will discuss, however, virtually all past methodologies are fraught with serious errors because the mathematics of the statistical fiber fragmentation process is highly complicated and not amenable to simple formulas unless sophisticated correction factors are applied (Henstenburg and Phoenix 1989; Hui et al., 1995, 1996). Actually, determining the fiber strength at the scale of the fiber fragment lengths is itself difficult, requiring at least a Weibull-weakest link model whose parameters in turn are difficult to determine at that length scale.

On the other side, there is the question of how such measured interfacial micromechanical information is to be used in predicting the behavior or large fibrous composite structures. Obviously tailoring of interfaces to have certain characteristics is a promising avenue to developing large-scale composite structures with such properties as high strength, acceptable toughness, creep- 
rupture resistance, fatigue resistance and high reliability (in terms of high design loads, but with extremely low probabilities of failure, e.g. less than one failure in a million). Unfortunately, models to make this link with the sort of detail being pursued in SFC tests or similar tests using a few fibers in a matrix are not available. (An exception is Goda and Phoenix, 1994). Moreover the conditions of such idealized micromechanical tests usually are not duplicated in commercial composites because in the latter fibers are closely spaced and matrix volume fractions are relatively low so that the matrix is highly constrained in how it can respond. In particular, the matrix carries almost no tension and is limited in how much relative fiber displacement it can accommodate before yielding or debonding, so the fiber stress profiles along broken fibers will differ from those in idealized SFC and multifiber specimens where the fiber spacing and matrix volume is typically much larger. Furthermore, in commercial composites, it is the stress profiles on overloaded fibers next to single or multiple fiber breaks that drives the composite failure process through progressive fiber breakage, and these situations are not usually captured in idealized tests, especially the SFC test. It is fair to say that virtually all models, whether in a local load-sharing framework or a global load-sharing framework among fibers, that attempt to predict the failure characteristics of larger multifiber composites with high fiber volume fractions, can presently use little more than a constant $\tau$ characterization of the interface near a fiber break, which in turn establishes a length scale for load transfer (Phoenix and Raj, 1992; Phoenix 1993; Phoenix et al., 1997). So beyond an effective $\tau$ value, how to use the interfacial information being pursued in idealized micromechanical tests such as the SFC test, is still quite unclear though some progress is being made in the literature (e.g. see Goda and Phoenix, 1994). In what follows we give brief descriptions of what appears on the transparencies of the talk. 
Transparency 1: This sketches the issues described above.

Transparency 2: This illustrates how in the SFC test the fiber fragmentation process is complicated involving slip or shielded zones that develop around breaks assumed to be given by the simple Kelly-Tyson slip formula. Also shown is the Weibull Poisson model for fiber strength with associated parameters. These two aspects lead to great complications in determining the fiber fragment length distribution from which one would 'back out' an effective $\tau$ value assuming it was unknown.

Transparency 3: This gives more detail on the fragmentation progression indicating that the process is related to the so-called parking problem when the Weibull fiber shape parameter $\rho$ is taken as infinite. For finite $\rho$ such analysis leads to establishing stress and length scales or 'normalization parameters' for the fragmentation process, thus simplifying interpretation.

Transparency 4: This gives the two normalization parameters in terms of the fiber Weibull parameters measured for a given gage length, the interfacial (constant) shear stress and the fiber radius (e.g. Hui et al., 1997).

Transparency 5: This shows various possibilities for idealizing the fiber surface, shear stress profiles (and associated matrix/interface deformation laws) around a fiber break for generating a statistical theory for fragmentation (from Hui et al., 1996).

Transparency 6: This shows the fiber stress profile (bilinear) and shear tractions for a two $\tau$ model including a small elastic decay driven zone, which may affect where subsequent breaks occur (from Henstenburg and Phoenix, 1989).

Transparency 7: This summarizes the Weibull fiber model for use in the SFC fragmentation analysis including potential initial breaks before loading, the exclusion zone length around a break (twice the slip length), the stress and length normalization parameters and the normalized fiber stress (Hui et al., 1995). 
Transparency 8: This begins several transparencies which describe the complexity of the fragmentation probability analysis. Two quantities introduced here are (i) the flaw frequency in terms of number of flaws originally existing in the fiber with normalized strength less than the normalized fiber stress $s$ and (ii) the hazard rate for encountering new flaws per unit length as the stress is increased (Hui et al., 1995).

Transparency 9: This describes the density function (not a probability density function in the usual sense) of the number per unit length of fragments of length exactly $x$ when the stress has reached the normalized stress $s$. The normalizing condition simply says that for a very long fiber, the fragments of various lengths have to add up to the total fiber length (Hui et al., 1995).

Transparency 10: This describes the system of integral-differential equations that must be solved to obtain the fiber fragment length distribution at every stress $s$. The starting condition requires a certain number of initial breaks (at zero load) of rate $\alpha$ along the fiber after which the desired result is obtained taking the limit as $\alpha$ goes to zero (Hui et al., 1995).

Transparencies 11 and 12: These describe the 'exact' closed-form solution for the fragment length density function that results from solving the differential equations. At a given stress level $s$, long lengths, medium lengths and short lengths all have their own special character (Hui et al., 1995).

Transparency 13: This describes two quantities of interest, namely the average number of breaks that occur per unit length at normalized stress $s$, and, the fraction of segments at that stress level with normalized length less than normalized length $x$ (Hui et al., 1995).

Transparency 14: Since most SFC tests take the fragmentation process to saturation this gives expressions for the final fragment length distribution, the latter showing that the lower tail of the cumulative fragment length distribution 
has shape parameter double the Weibull fiber shape parameter plus one (Hui et al., 1997).

Transparency 15 and 16: This plots the normalized mean fragment length against the normalized applied stress level in the SFC test together with two approximations. The Weibull shape parameter for fiber strength is the relatively low value of 3 . Note that the two approximations fail to capture the saturation phenomenon that sets in around $s=1$. The second transparency points out the fact that the failure of the two approximations is tied to their inability to capture the exclusion zones that develop and grow around fiber breaks, which eventually cover the whole fiber (Hui et al., 1997).

Transparency 17: This figure demonstrates that details of the fragmentation phenomenon are highly sensitive to the value of the Weibull shape parameter $\rho$ for fiber strength, and that convergence to deterministic strength (parking problem) models ( $\rho$ equals infinity) in terms of large shape parameter values is surprisingly slow. Thus, such limiting results are not very useful (Hui et al., 1997).

Transparency 18: A plot of the normalized mean number of breaks per unit length versus normalized stress against two approximations. The GulinoPhoenix approximation, a relatively simple result, works quite well up to near saturation, but then overcompensates for the growing exclusion zones which are treated as non-overlapping (Hui et al., 1997; Gulino and Phoenix, 1991).

Transparency 19: This figure shows that the normalized length distribution (cumulative) is not straight on Weibull coordinates even at saturation. It does not follow any of the common distribution forms (exponential, normal, lognormal, gamma etc.) (Hui et al., 1997).

Transparency 20: This figure shows that fiber fragmentation data along the way to saturation, treated as fraction of final saturation number of failures 
versus stress level, gives an empirical distribution function, which is surprisingly straight on Weibull coordinates (Henstenburg and Phoenix, 1989). The dotted lines are from Monte Carlo simulation of the problem. This yields a scheme for estimating the Weibull parameters for fiber strength as though the fibers were tension tested at a gage length approximately the mean fragmentation length. Small corrections for the shape parameter values are required.

Transparency 21: This transparency shows how the distribution of final fragment lengths at saturation changes with the Weibull shape parameter for fiber strength. The plots are on Weibull coordinates (Hui et al., 1995).

Transparency 22: This transparency illustrates fracture patterns for two very different load-sharing schemes., 'equal- or global load-sharing' where a broken fiber redistributes its load uniformly onto all non-failed or slipping fibers in a cross-section, versus 'local load-sharing', where a broken fiber redistributes its load laterally onto its nearest neighbors (Phoenix and Raj, 1992). In either case most statistical fracture models use only the simplest interfacial stress profile information, largely to establish the length scale of load transfer. Much work is necessary to be able to use the more sophisticated information sought in SFC and other related tests with several fibers.

Transparency 23: A pictorial of fiber failure activity around a crack tip, and the opportunity potentially to incorporate the interfacial information being sought.

Transparency 24: An idealized model of yield and debond zones around a fiber break in a composite. The objective in this and subsequent transparencies is to address stress profile and energy release rate issues with increased fiber spacing (Beyerlein and Phoenix, 1997).

Transparency 25: A constitutive law for how the combination of the matrix and interface between the fibers responds in shear. The axes are 
normalized stress versus normalized effective local strain in the matrix-interface combination (Beyerlein and Phoenix, 1997).

Transparency 26: An idealized view of the zones that might develop in the matrix between fibers if the fiber spacing is large enough for the matrix only to yield locally in a sheath around the broken fiber Beyerlein and Phoenix, 1997).

Transparency 27: Three fiber spacings (i.e. fiber volume fractions) and associated schematics of elastic and plastic zones as motivated by photoelastic studies. No debonding is assumed (Beyerlein and Phoenix, 1997).

Transparency 28: Energy release rates from global calculations for growing plastic and debond zones for various frictional stresses after debonding. When debond frictional stresses are large the required energy release rate to propagate the debond grows roughly linearly with the debond length. When the frictional stress is approximately zero, this energy is roughly constant with debond length and comes from plastic energy dissipation in the matrix up to debonding (Beyerlein and Phoenix, 1997).

Transparency 29: This figure illustrates that the energy release rate for the three cases in Transparency 27 differs despite the fact that the basic mechanical properties of the matrix and interface (unnormalized) are the same, the main difference being the local composite geometry. This suggests that energy release rates from SFC's and other idealized micromechanical experiments are not likely to reflect what actually happens in a real composite and that the local details of the fiber, matrix and interface stresses and strains are important (Beyerlein and Phoenix, 1997).

Transparency 30: This figure illustrates the fact that plastic zones and debond lengths around fiber breaks depend on the spacings of fiber breaks (Beyerlein and Phoenix, 1997). 
Transparency 31: This figure shows how the fiber stress profile varies for the situation of Transparency 30. These profiles are what microRaman spectroscopy measures from which one can back calculate the stresses at the interface and the lengths of the yield and debond zones. Recent work indicates that the view presented is reasonably accurate (Beyerlein and Phoenix, 1997).

\section{References}

I. J. Beyerlein and S. L. Phoenix (1997). "Stress profiles and energy release rates around fiber breaks in a lamina with propagating zones of matrix yielding and debonding", Composites Science and Technology, 57, 869-885.

K. Goda and S. L. Phoenix (1994). "Reliability approach to the tensile strength of unidirectional CFRP composites by Monte-Carlo simulation in a shear-lag model", Composites Science and Technology, 50, 457-468.

R. Gulino and S. L. Phoenix (1991). "Weibull strength statistics for graphite fibers measured from the break progression in a model graphite/glass/epoxy composite", Journal of Materials Science, 26, 3107-3118.

R. B. Henstenburg and S. L. Phoenix (1989). "Interfacial shear strength studies using the single-filament-composite test II: a probability model and Monte-Carlo simulation", Polymer Composites, 10, 389-408.

C.-Y. Hui, S. L. Phoenix, M. Ibnabdeljalil and R. L. Smith (1995). "An exact closed form solution for fragmentation of Weibull fibers in a single filament composite with application to fiber reinforced ceramics", J. Mechanics and Physics of Solids, 43, 1551-1585.

C.-Y. Hui, S. L. Phoenix and L. Kogan (1996). "Analysis of fragmentation in the single filament composite: roles of fiber strength distributions and exclusion zone models", Journal of the Mechanics and Physics of Solids, 33, 2543-2574.

C.-Y. Hui, S. L. Phoenix and D. Shia (1997). "The single filament composite test: Application of new statistical theory for estimating $t$ and the Weibull parameters for fiber strength". Composites Science and Technology, to appear.

J. A. Nairn and Y. C. Liu (1997). "Stress transfer into a fragmented, anisotropic fiber through an imperfect interface", Int. J. Solids and Structures, to appear 
S. L. Phoenix, M. Ibnabdeljalil and C.-Y. Hui (1997). "Size effects in the distribution for strength of brittle matrix fibrous composites", Int. J. Solids and Structures, 34, 545-568.

S. L. Phoenix and R. Raj (1992). "Scalings in fracture probabilities for a brittle matrix fiber composite", Acta Metallurgica et Materialia, 40, 2813-2828.

S. L. Phoenix (1993). "Statistical issues in the fracture of brittle matrix fibrous composites", Composites Science and Technology, 48, 65-80.

L. S. Schadler and C. Galiotis (1995). "A Review of the fundamentals and applications of laser Raman spectroscopy microprobe strain measurements in composite materials", J. International Materials Reviews, 50, 116-134. 
Statistical

$\rightarrow$ Analysis of Data From

Single Filament Composite Test) and Related Micromechariical Issues

Leigh Phoenix

T\&AM

Cornell University

- using the SFC test to measure

- ' $\tau$ ' (what is it?)

- Weibull fiber strength para meters - inter face constitutive laws - interface energy (surface, release,

- composite performance (or what th. h do we do with it all!

Transparency 非

95 


$$
\begin{aligned}
& \text { Kelly -Tyson slip length } \\
& l_{s}=d \sigma /\left(\begin{array}{c}
4 \tau) \\
+ \text { far field fiber }
\end{array}\right.
\end{aligned}
$$

Sample gauge length

a)

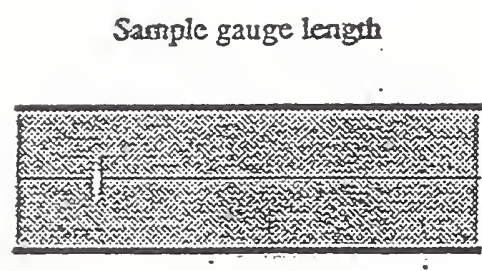

b)

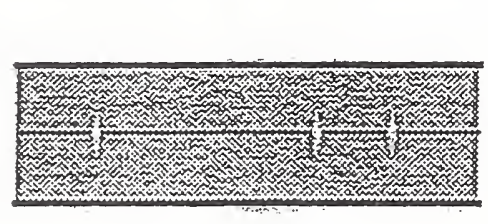

c)

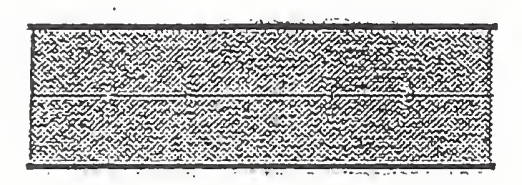

Stress profiles
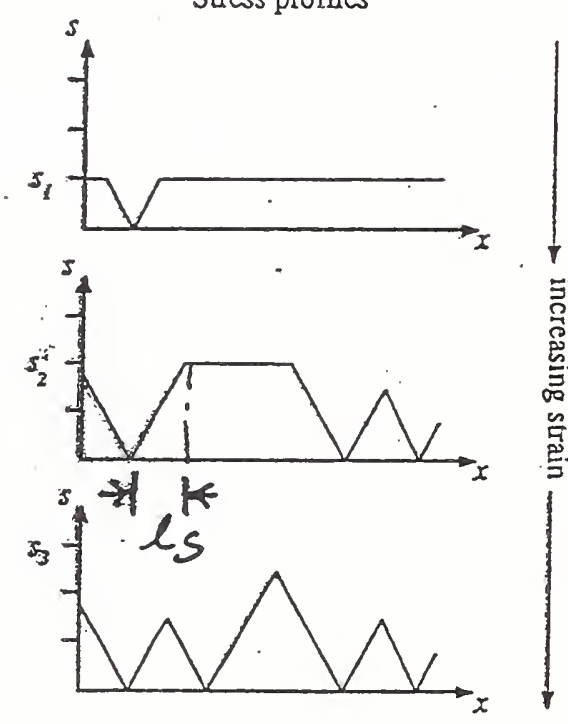

stress

Figure 2: The prog fission of fiber fragmentation during a s.f.c. test assuming a linear stress recovery around breaks.

Fiber strength dist

$$
F(\sigma)=1-e^{-l \Lambda(\sigma)}
$$

tension

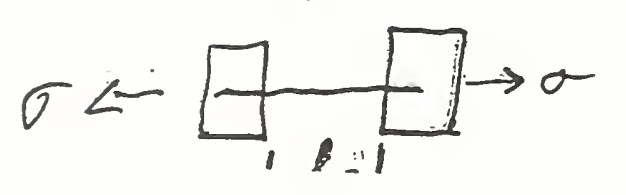

scale@reflengthlo

Transparency 非

96 
- establishes length stress scales and of spacing statistics

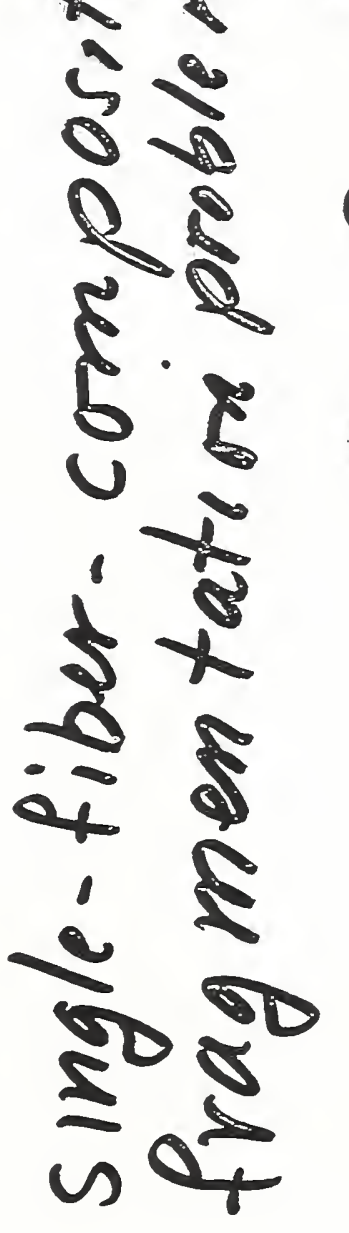

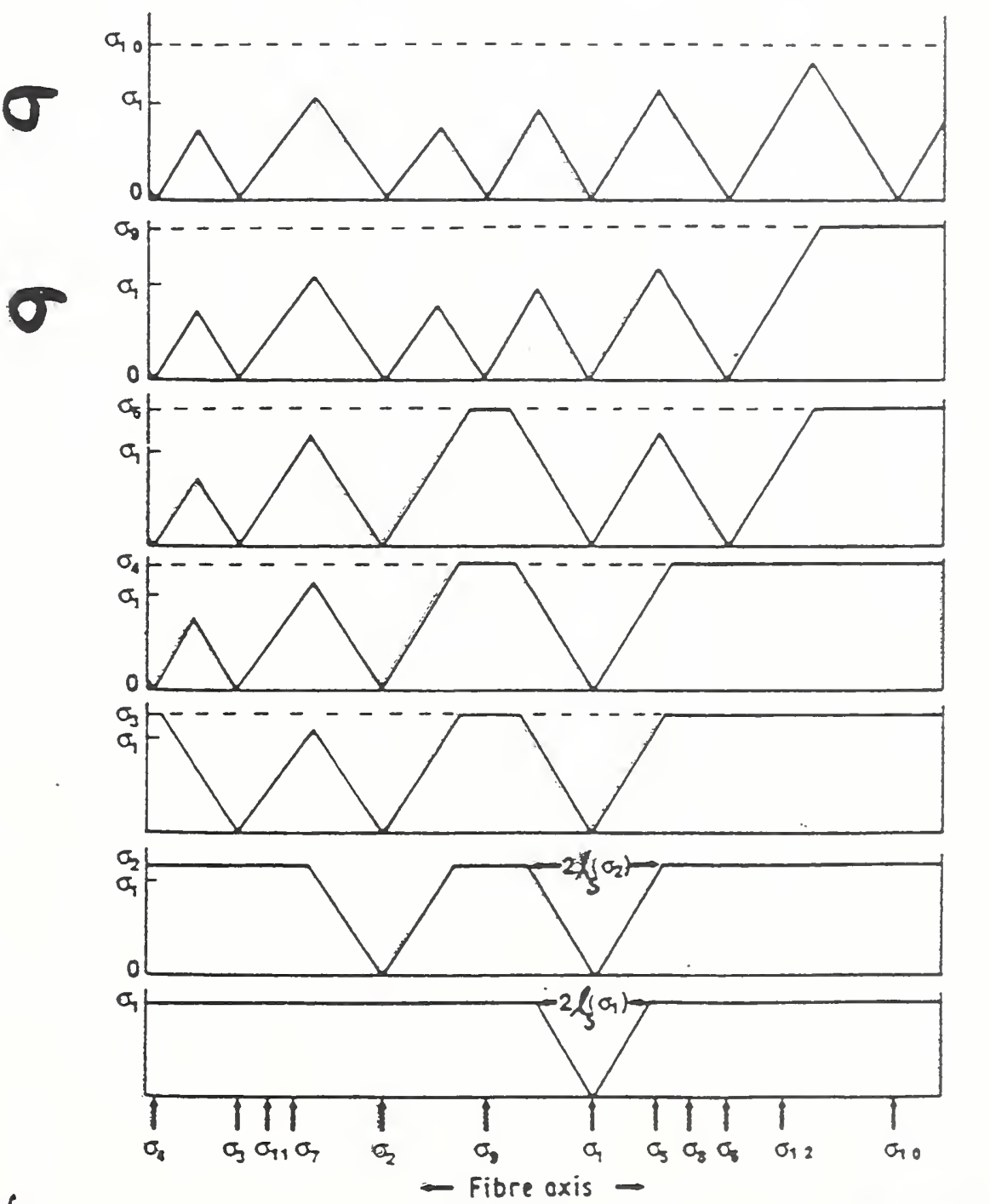
increases
$\sigma$
$\uparrow$

Parking problem $(\rho \rightarrow \infty$ Curtin (199/) Henstenburg Phoenix 19 . 


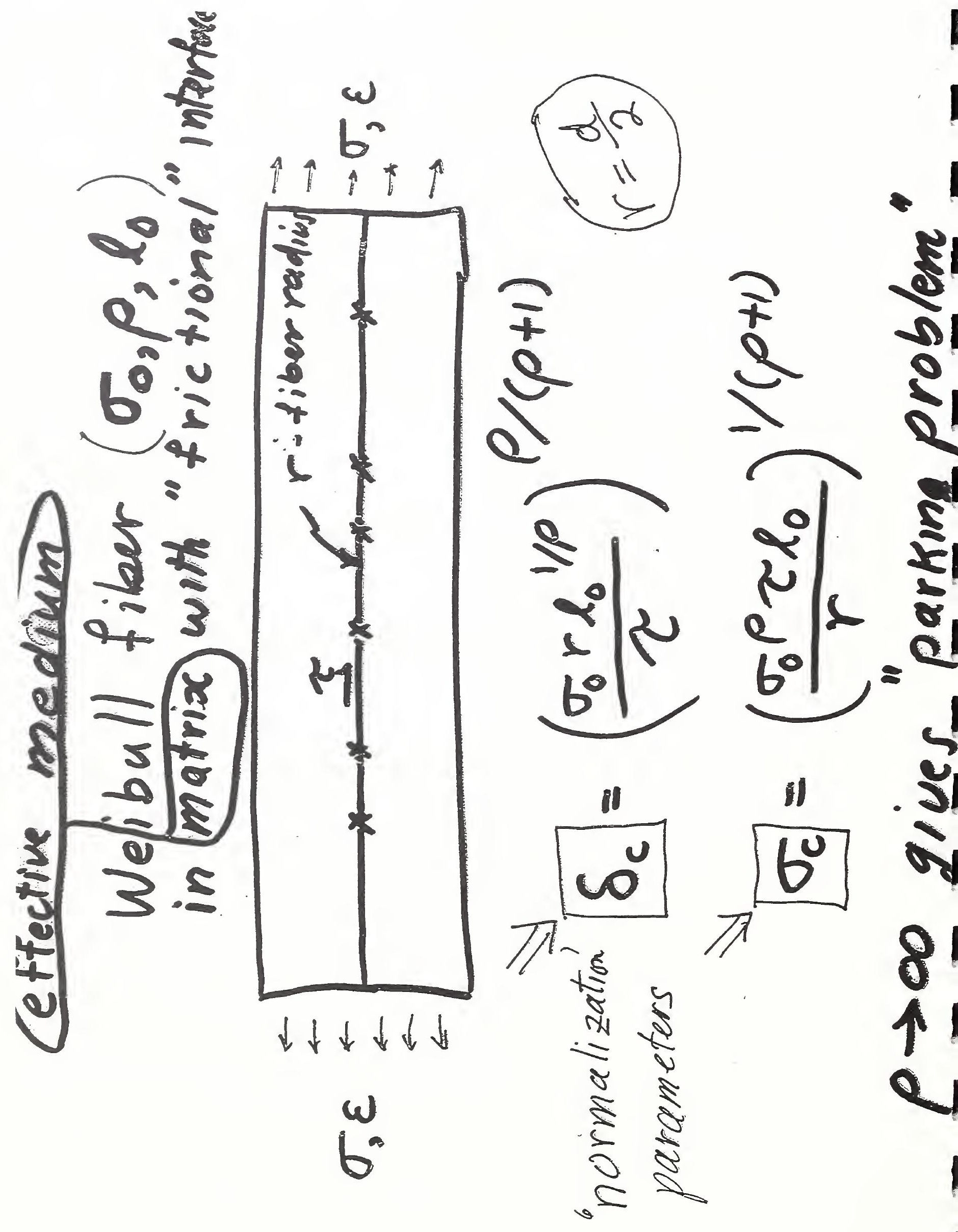




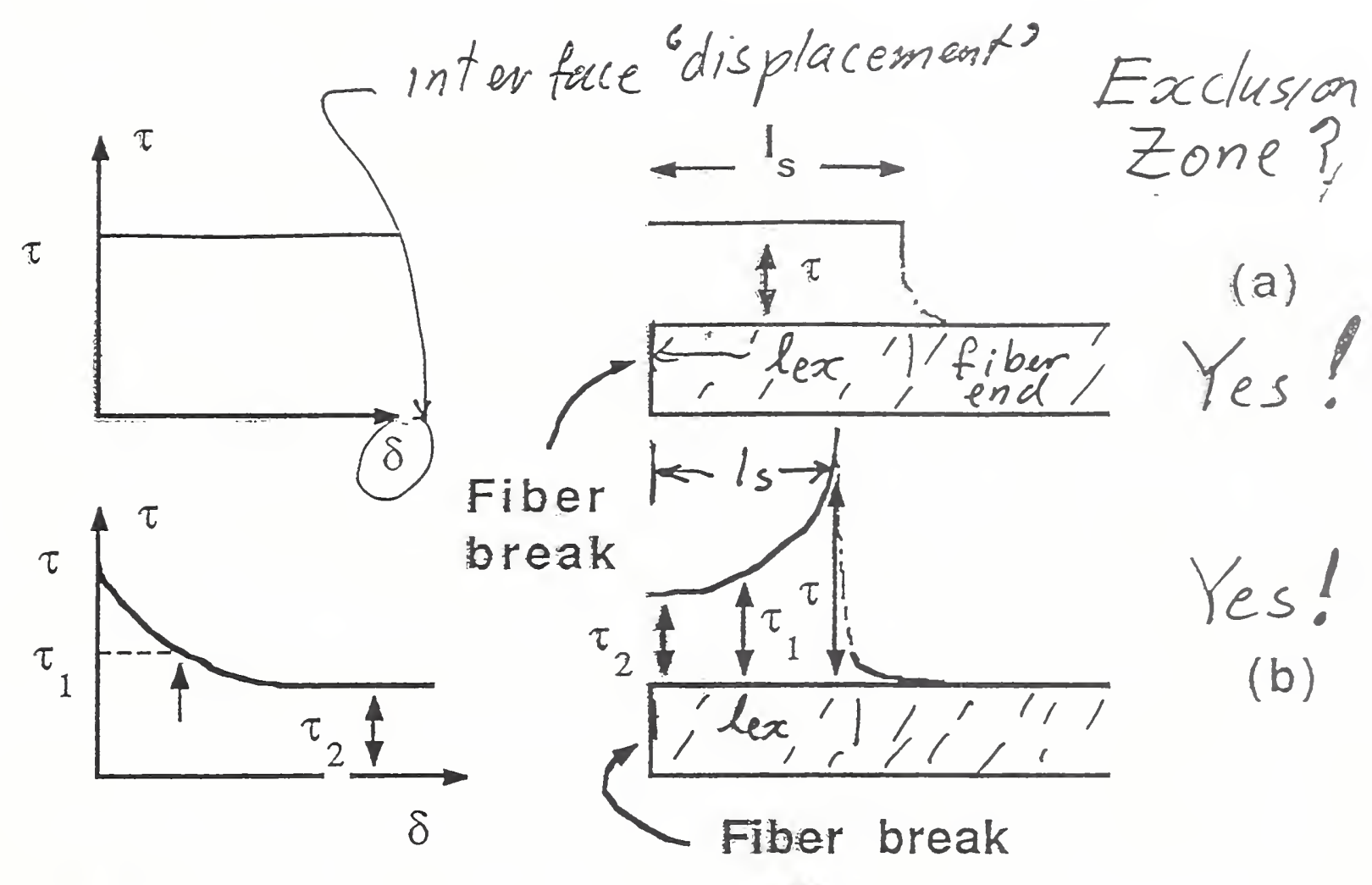

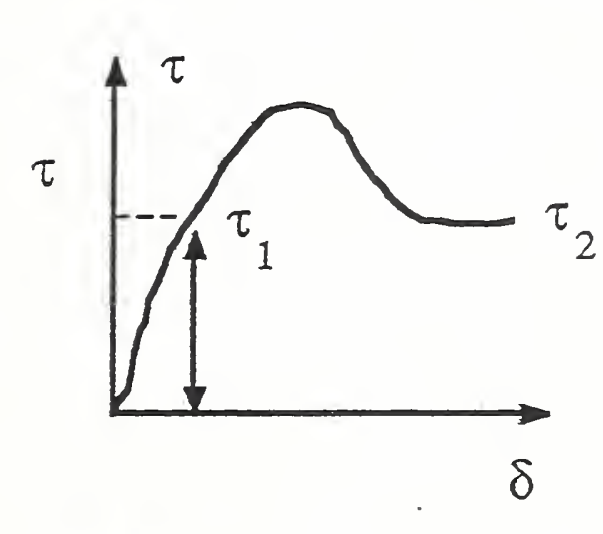

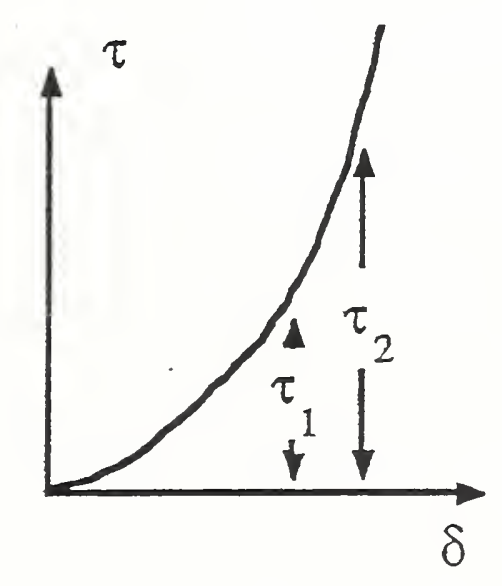

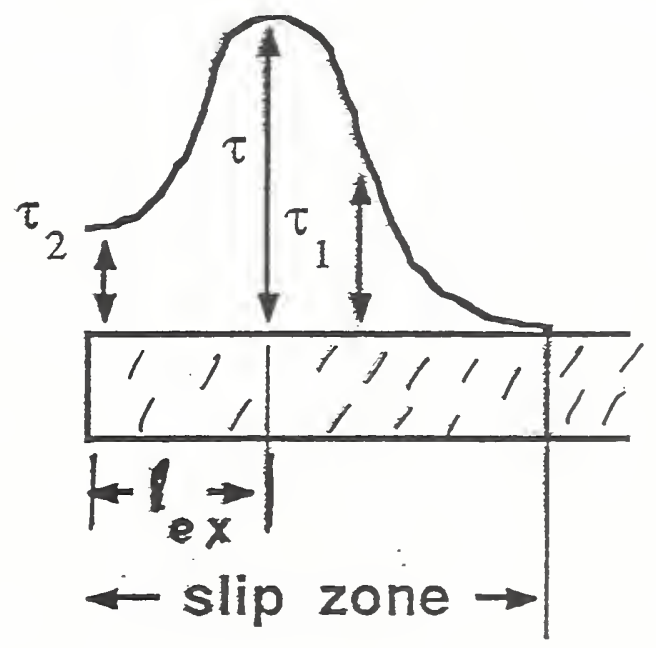

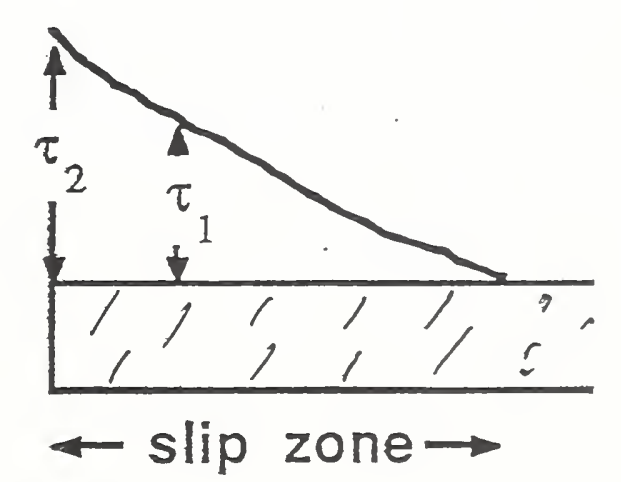

Sort of 1

(c)

No!

(d)

Figure 2

Other matrix/ interface deformakon l aus

Transparency 非 5

99 


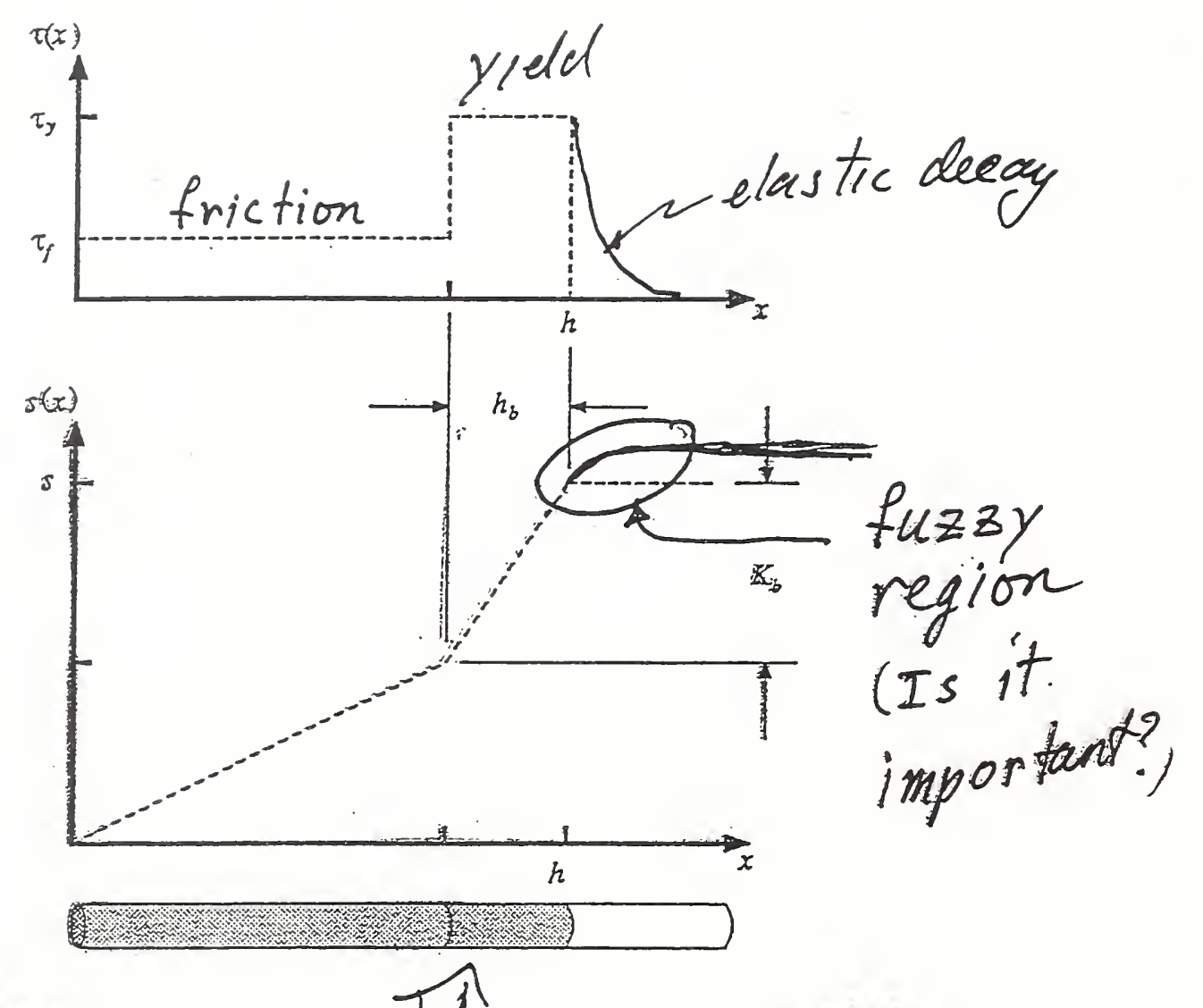

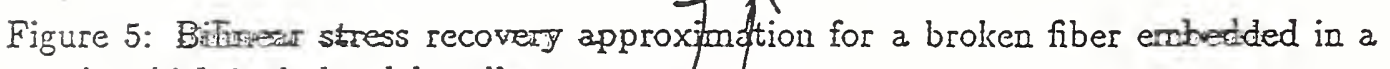
matrix which includes debonding.

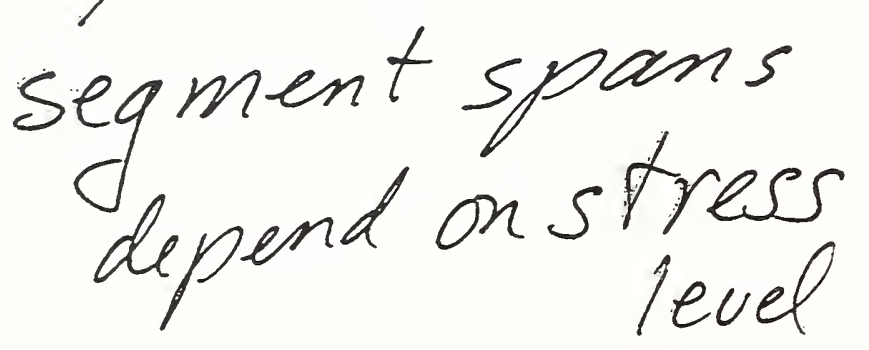

Transparency 非

100 


\section{Statistics of Fragmentation}

Basic Quantities

$$
\delta=d \sigma / 2 \tau
$$$$
\text { 'initial breaks' at zero }
$$$$
\Lambda\left(\sigma / \sigma_{0}\right)=\left[\alpha_{o}+\left(\sigma / \sigma_{0}\right)^{\rho}\right] / l_{0}
$$

$$
F(\sigma, l)=1-\exp \left[-l \Lambda\left(\sigma / \sigma_{0}\right)\right]
$$

normalized

stress

$\sigma_{c}=\sigma_{o}\left(2 l o l d \sigma_{o}\right)^{\lambda / \rho}$

$$
\lambda=\rho /(1+\rho)
$$

$$
\delta_{C}=l_{o}\left(d \sigma_{o} / 2 l_{o} \tau\right)^{\lambda} .
$$


normalise all lengths by $\delta_{c}$,

mean number of flaws per dimensionless length at dimensionless stress $s$ is

$$
\begin{aligned}
& \tilde{\Lambda}(s)=\alpha+s^{\rho} \\
& \alpha=\alpha_{o} /\left(\sigma_{c} / \sigma_{o}\right)^{\rho} .
\end{aligned}
$$

shielded region is $s / 2$ on each side of a break

hazard rate is

$$
h(s)=d \tilde{\Lambda}(s) / d s=\rho s^{\rho-1} \quad s>0 .
$$


$p(s, x) \quad$ - density function of the number per unit length of inter-break spacings or fragments of length $x$ at stress $s$.

normalizing condition

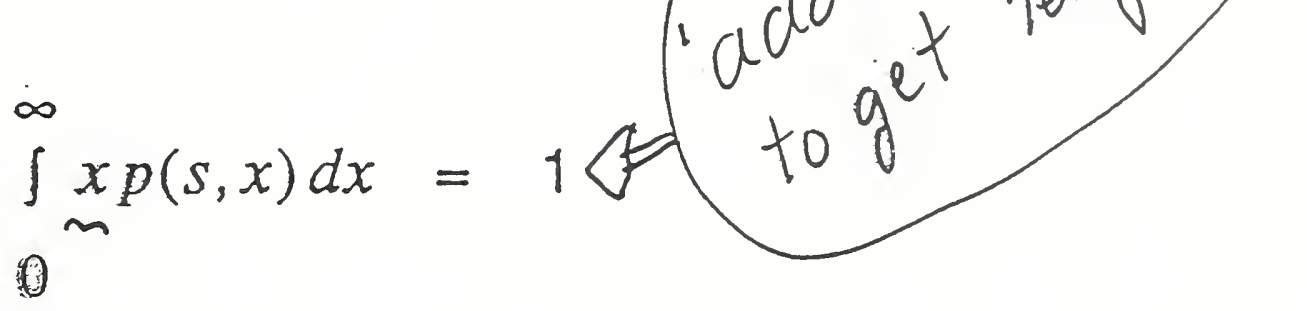


Governing equations for $p(s, x), \alpha>0, \rho>0$.

$$
\begin{aligned}
& \partial p / \partial s=0, \quad 0<x<s / 2 \text { Can'tchong } \\
& \partial p / \partial s=2 h(s) \int^{\infty} p(s, t) d t, \quad s / 2<x<s \\
& x+s / 2
\end{aligned}
$$

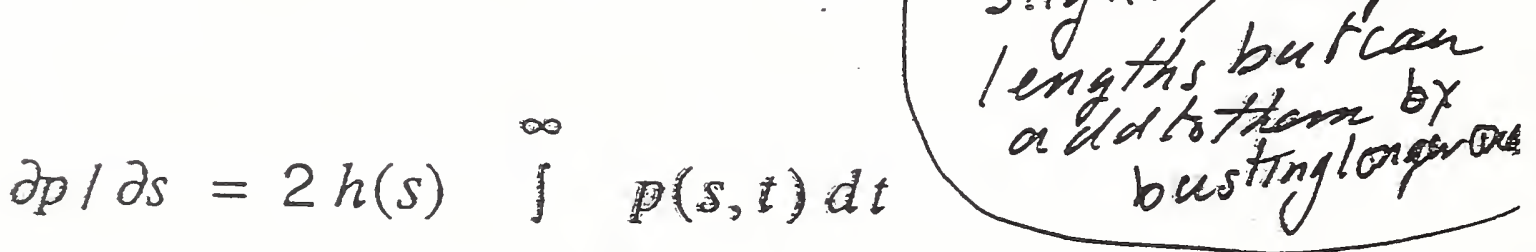

$$
\begin{aligned}
& x+s / 2 \\
& \text { - }(x-s) p(s, x) h(s), s<x \\
& \text { can't bust } \\
& \text { slightly longer } \\
& \rightarrow \text { iongust frag's } \\
& \text { can be busted } \\
& p(s=0, x)=\alpha^{2} \exp (-\alpha x) .
\end{aligned}
$$


After 30 yrs

Exact closedform solution $\alpha>0, \rho>0$.

$$
p(s, x)=A(s) \exp (-\beta(s) x), \quad s<x
$$

find

$$
\begin{aligned}
& \beta(s)=s^{\rho}+\alpha . \\
& A(s)=\alpha^{2} \exp \left(\lambda s^{\rho+1}\right) \exp \left[2 H\left(s^{\rho}, \rho\right)\right]
\end{aligned}
$$

where

$$
H\left(s^{\rho}, \rho\right)=\int_{0}^{s^{\rho}} \frac{\exp \left[-(t+\alpha) t^{1 / \rho} / 2\right]}{t+\alpha} d t
$$


then

$$
\begin{aligned}
& p(s, x)=A(s) \exp [-\beta(s) x], \quad s<x \\
& p(s, x)=\int_{x}^{s} \frac{2 h(t) A(t)}{\beta(t)} \exp [-\beta(t)(x+t / 2)] d t \\
& +p(x, x), \quad s / 2<x<s \\
& p(s, x)=\int_{x}^{2 x} \frac{2\left(z_{2}(t) \cdot A(t)\right.}{\beta(t)} \exp [-\beta(t)(x+t / 2)] d t \\
& +(x, x), \quad x<s / 2 \\
& p(x, x)=\alpha^{2} \exp \left[\frac{-x^{\rho+1}}{1+\rho}\right] \exp \left[2 H\left(x^{\rho}, \rho\right)\right] e^{-\alpha x}
\end{aligned}
$$


Evaluation of $\chi(s)$ and $F(s, x)$ for $\alpha=0$

mean number of breaks per unit length

$$
\chi(s)=\int_{0}^{\infty} p(s, x) d x
$$

fraction of segments with length less than $x$

$$
F(s, x)=\int_{0}^{x} p(s, t) d t / \int_{0}^{\infty} p(s, t) d t
$$


Limiting distribution $\quad F(x)$

fraction $F(x)$ of fiber segments with length less than $x$ at saturation is

$$
\begin{aligned}
& F(x)=\lim _{s \rightarrow \infty} F(s, x) . \\
& F(x) \sim \frac{(2 x)^{2 \rho+1}}{2(2 \rho+1) \chi(\infty, \rho)}, \quad x<1 / 2,
\end{aligned}
$$




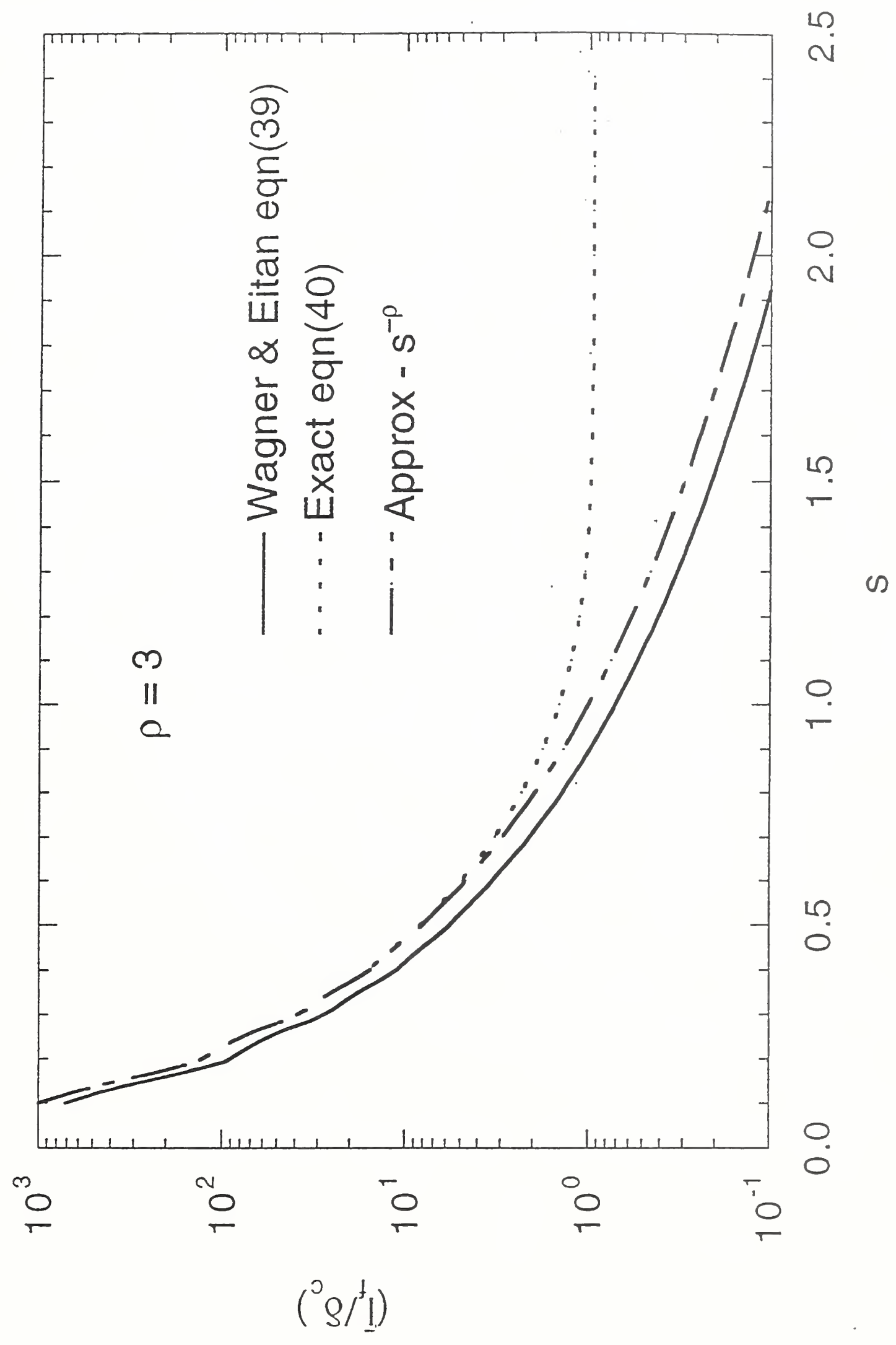

Transparency 非15 


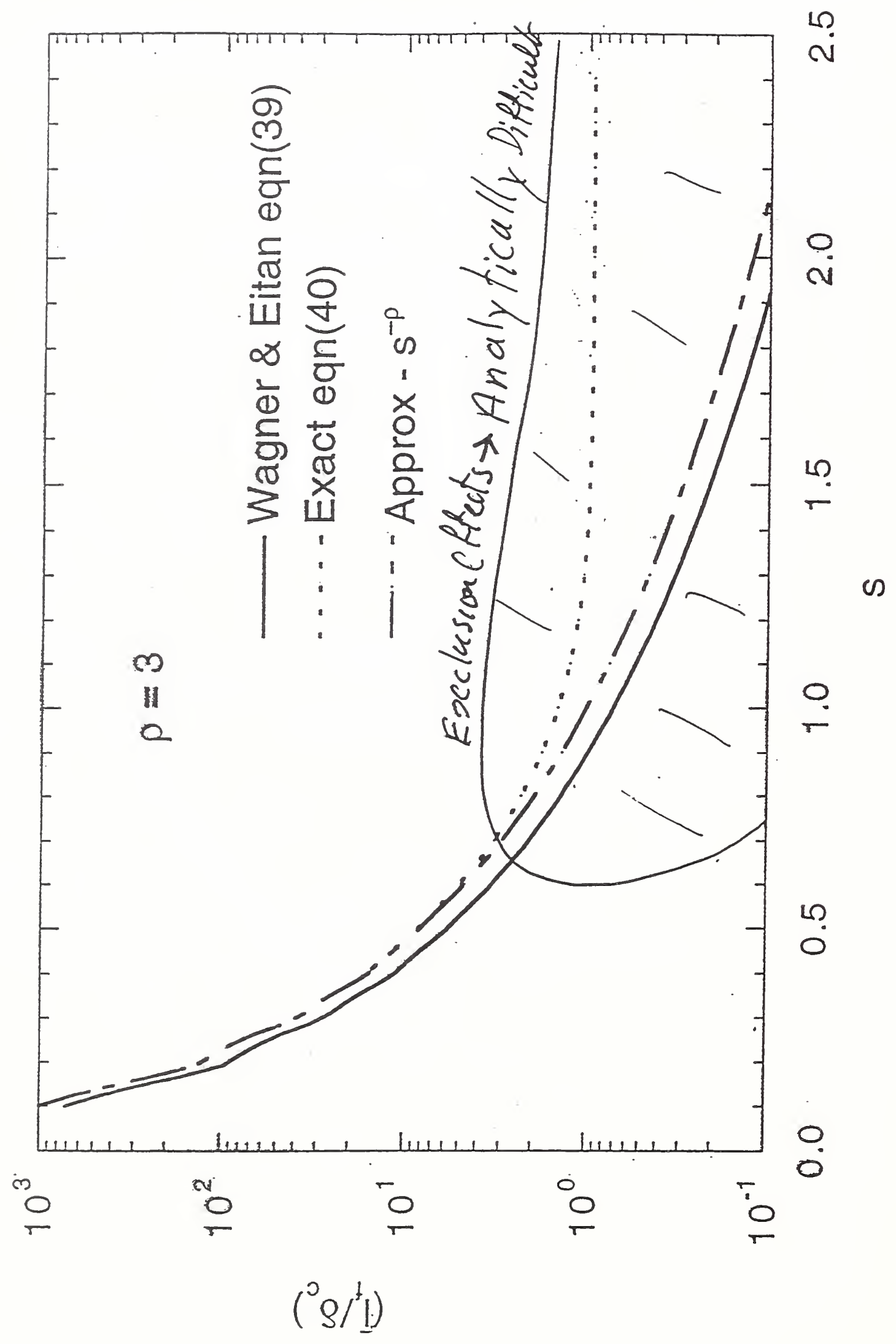

Transparency 非16 

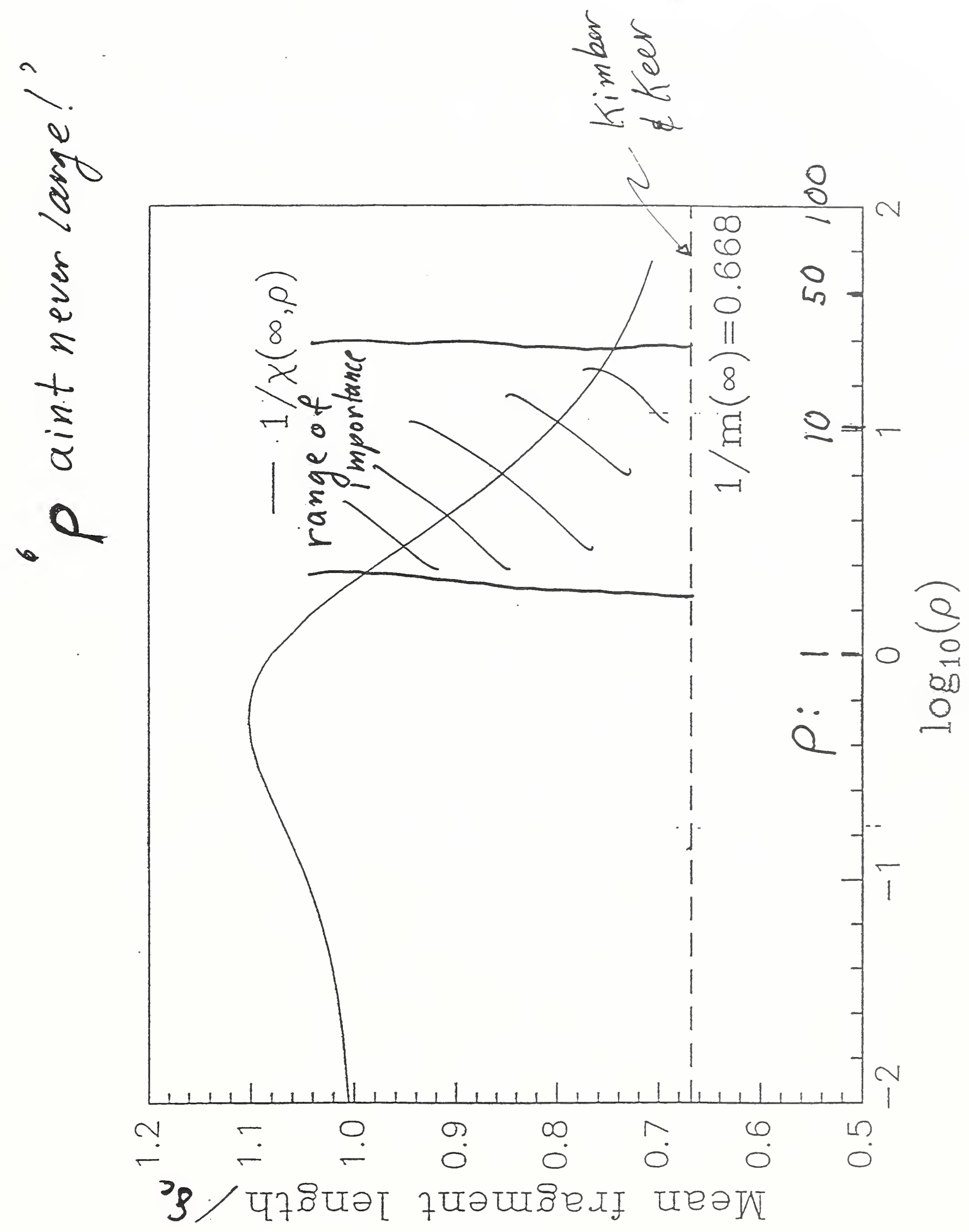

Transparency 非17 


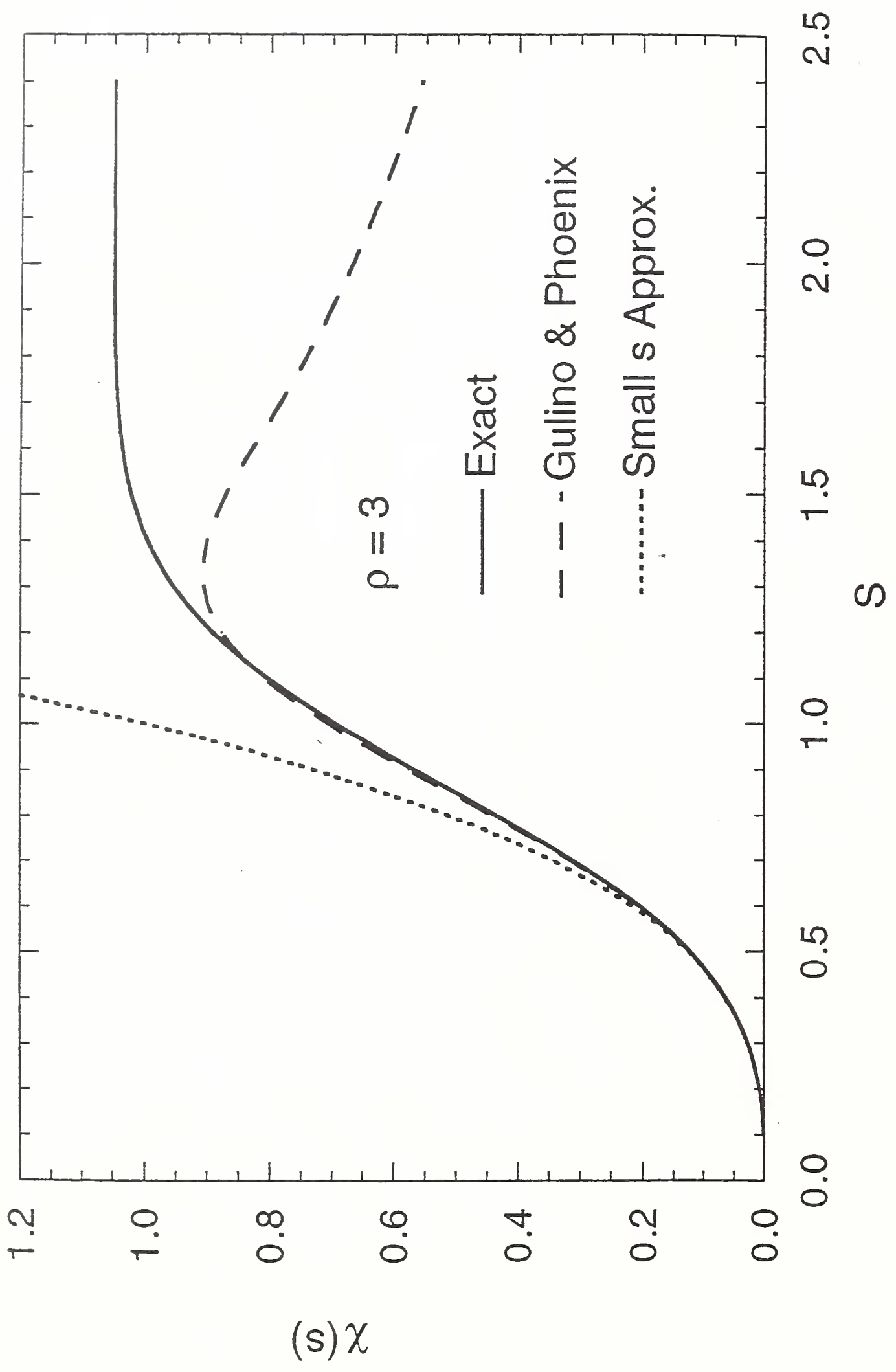

Transparency 非18 


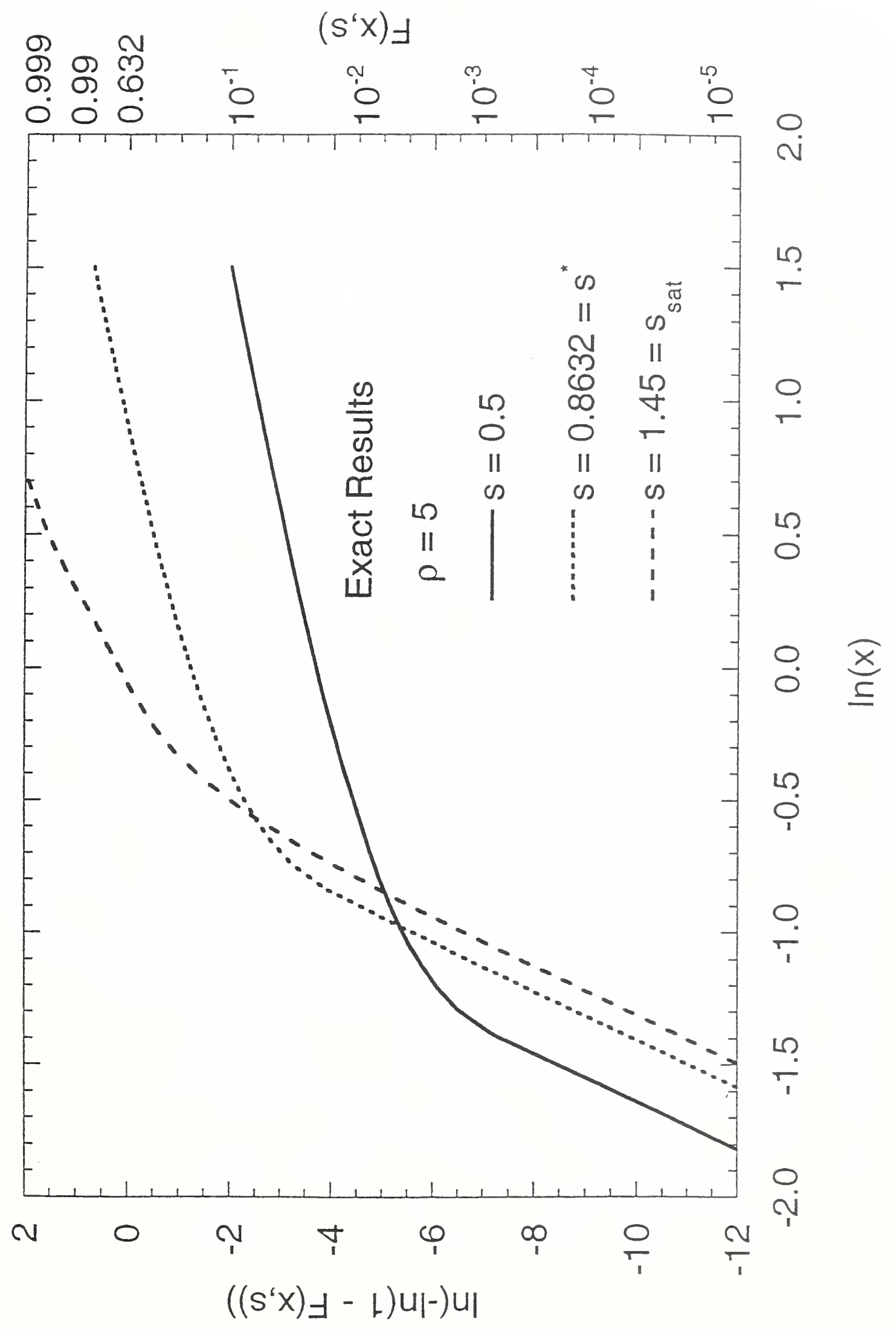

Transparency 非19 
Ln(Strength)

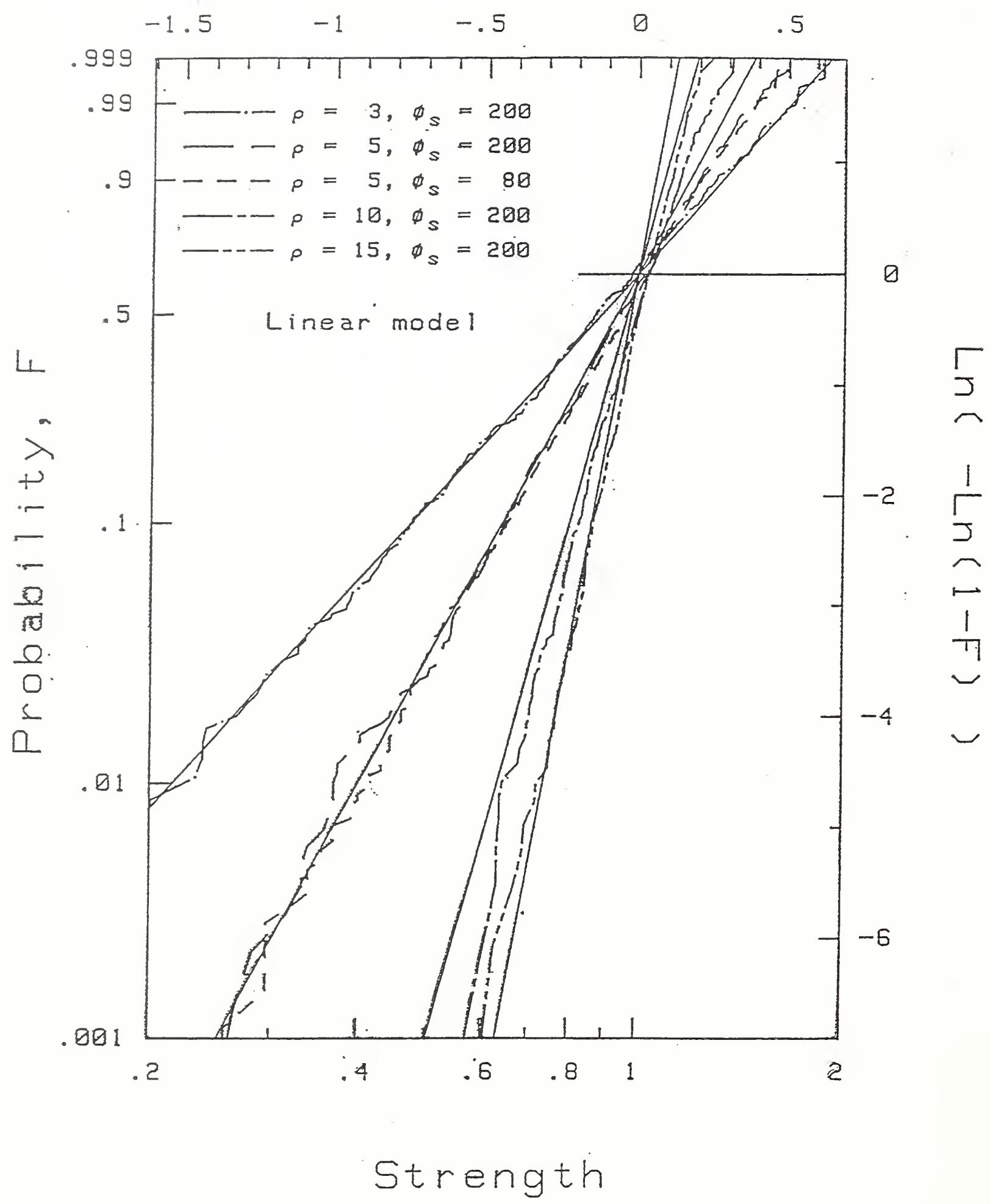

Transparency 非20 


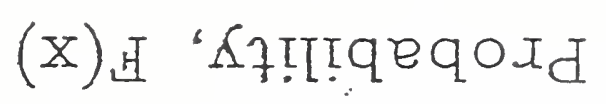

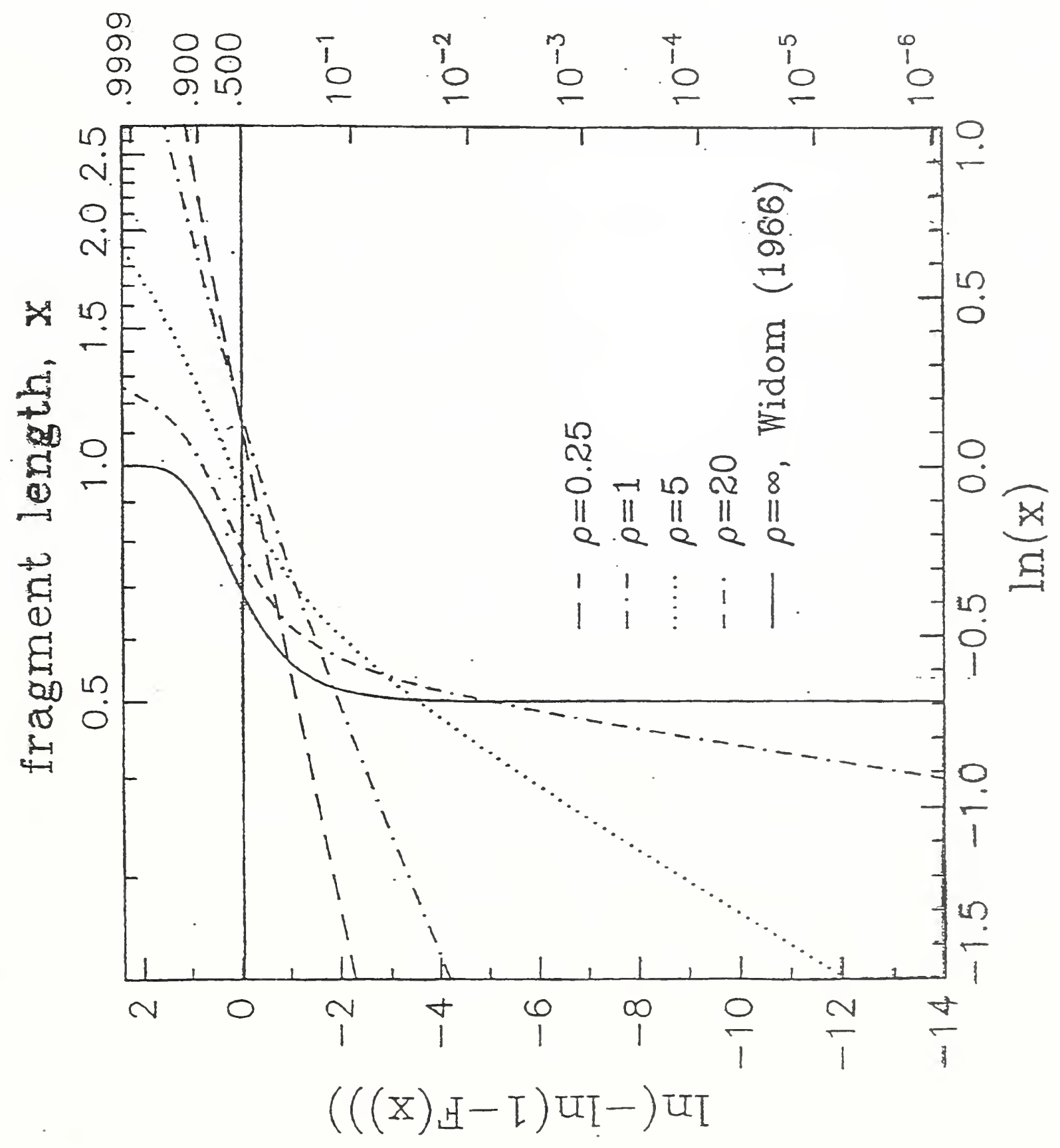

Transparency 非21 
D91-108
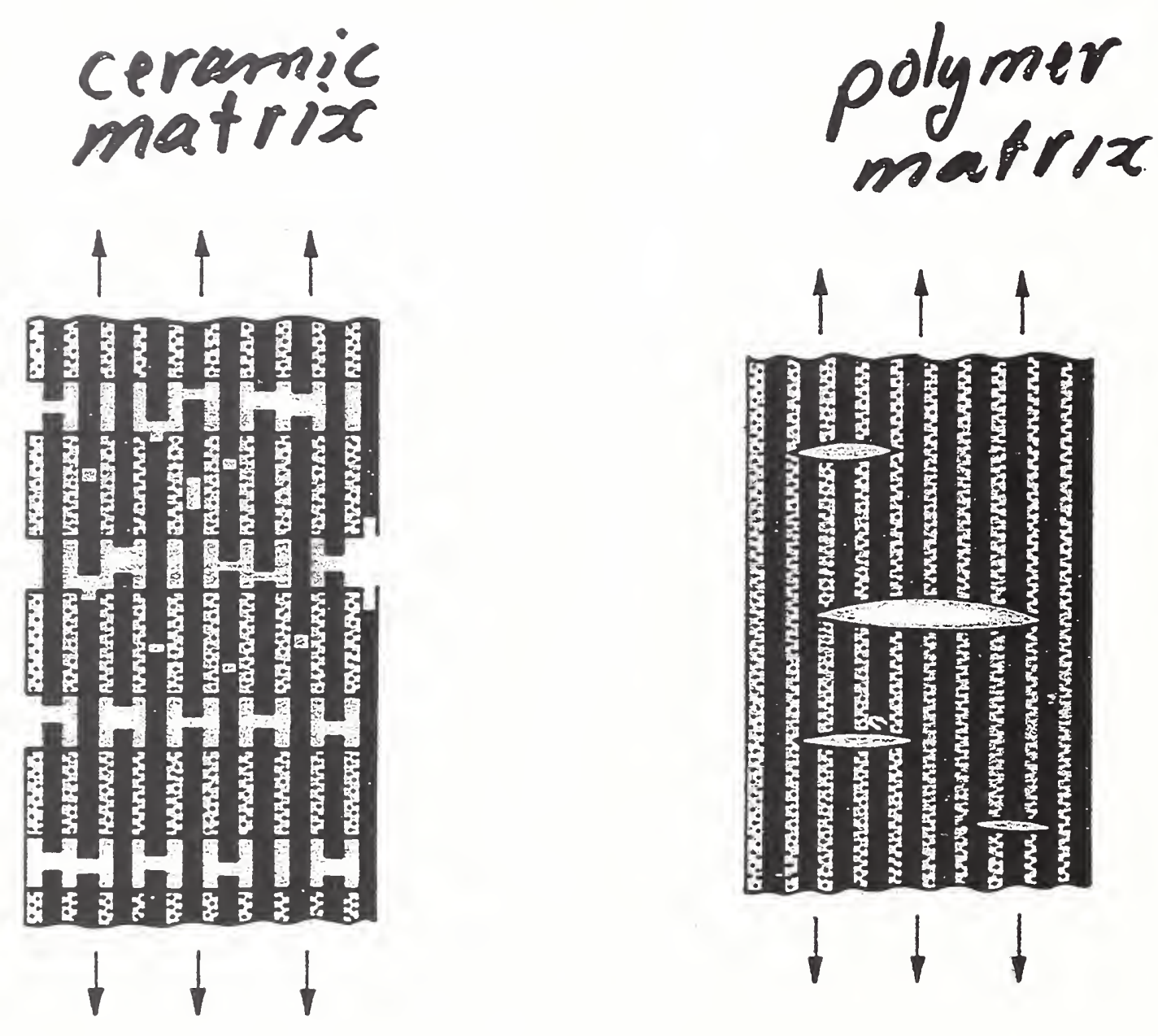

EQUAL

LOAD SHARING

LOAD SHARING 


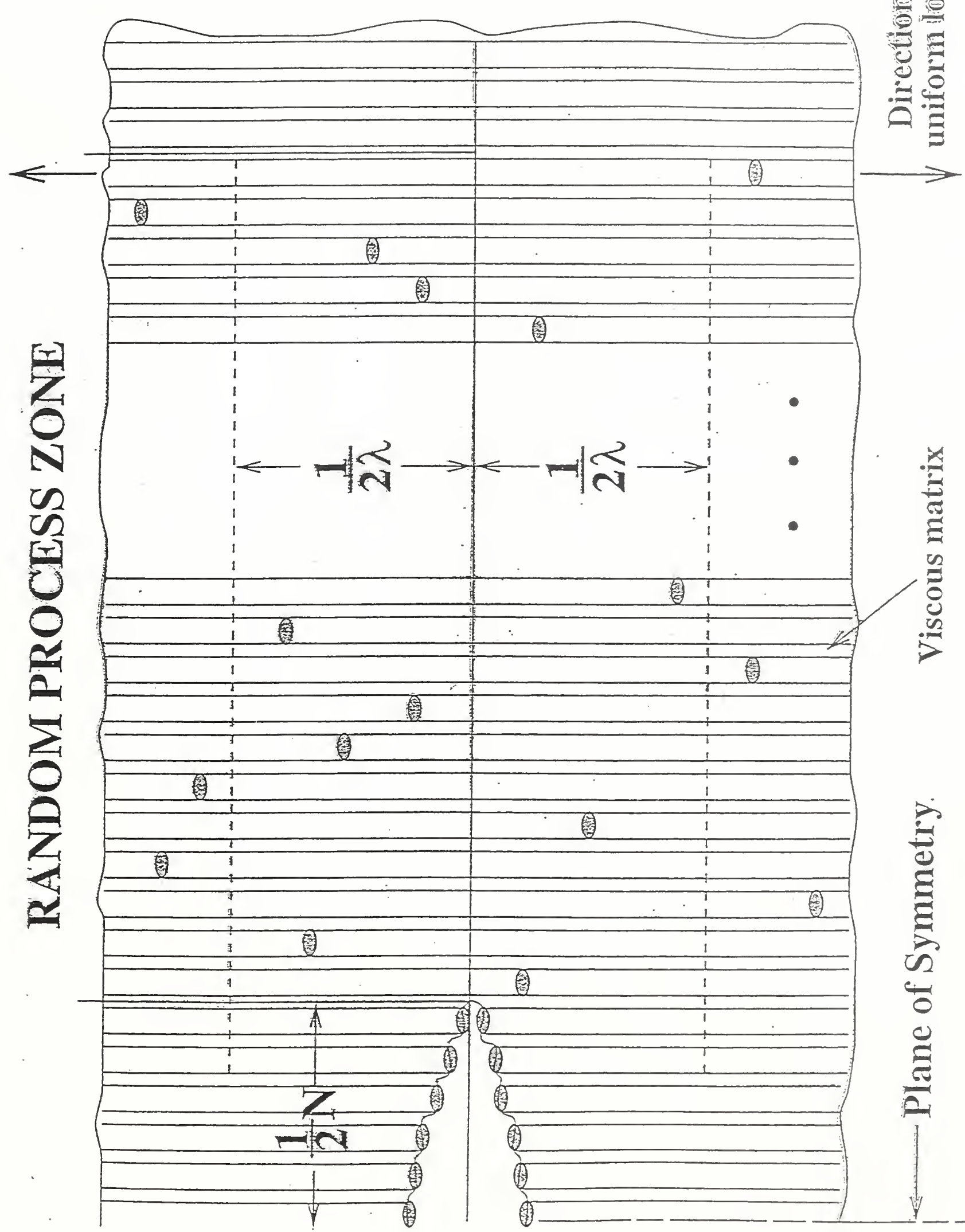

Transparency 非23 


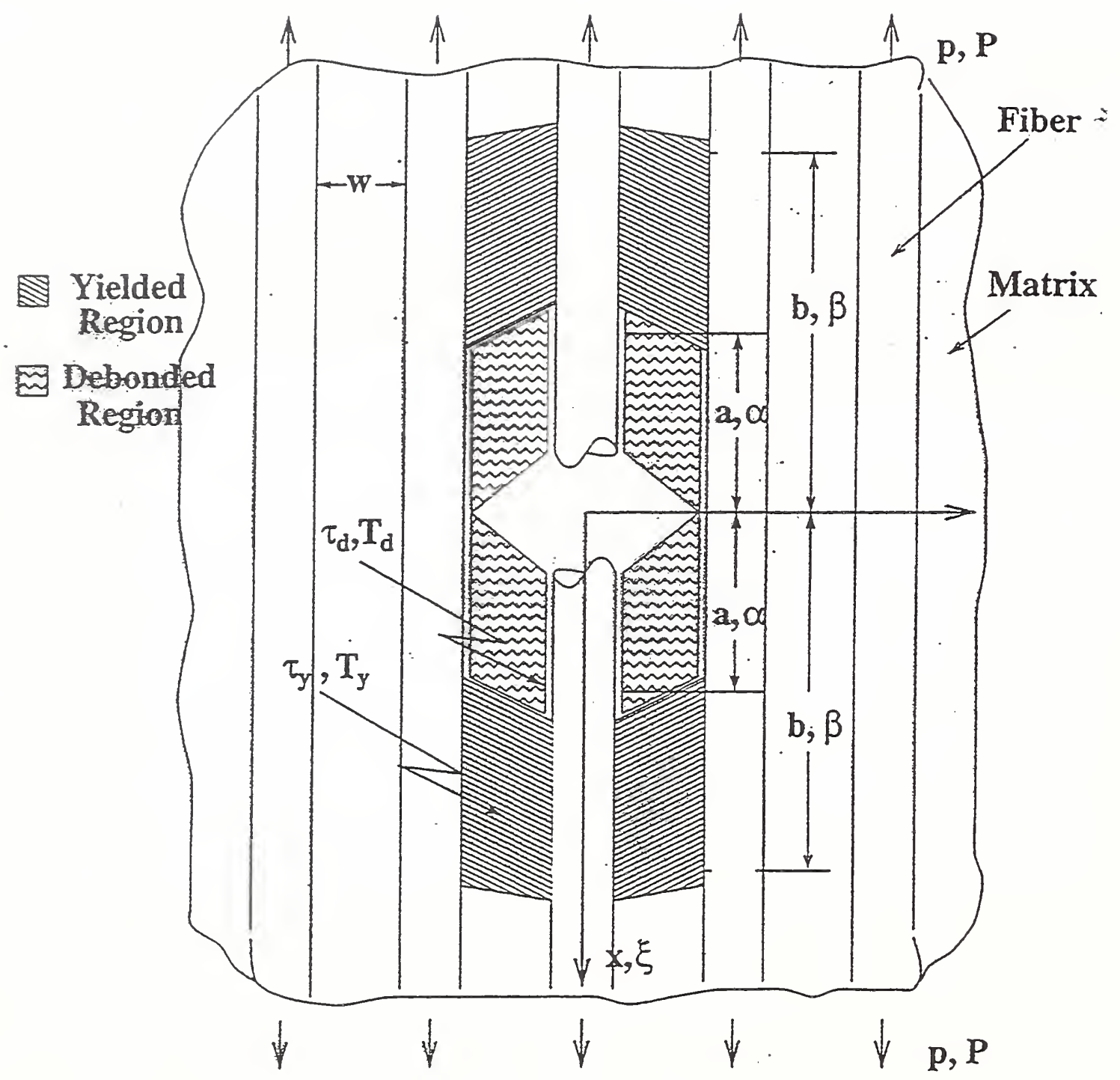

Transparency 非24 


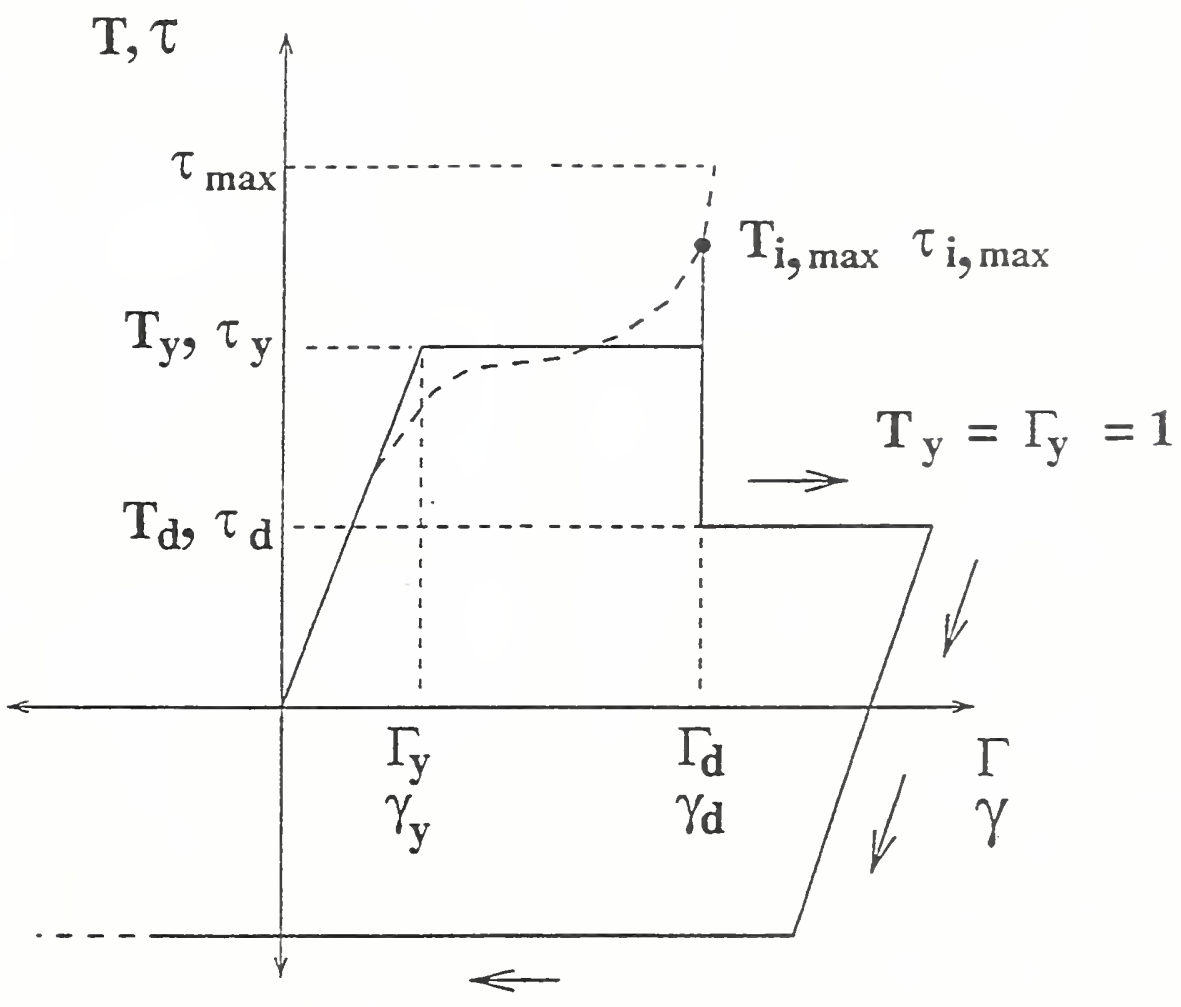

Transparency 非 25 


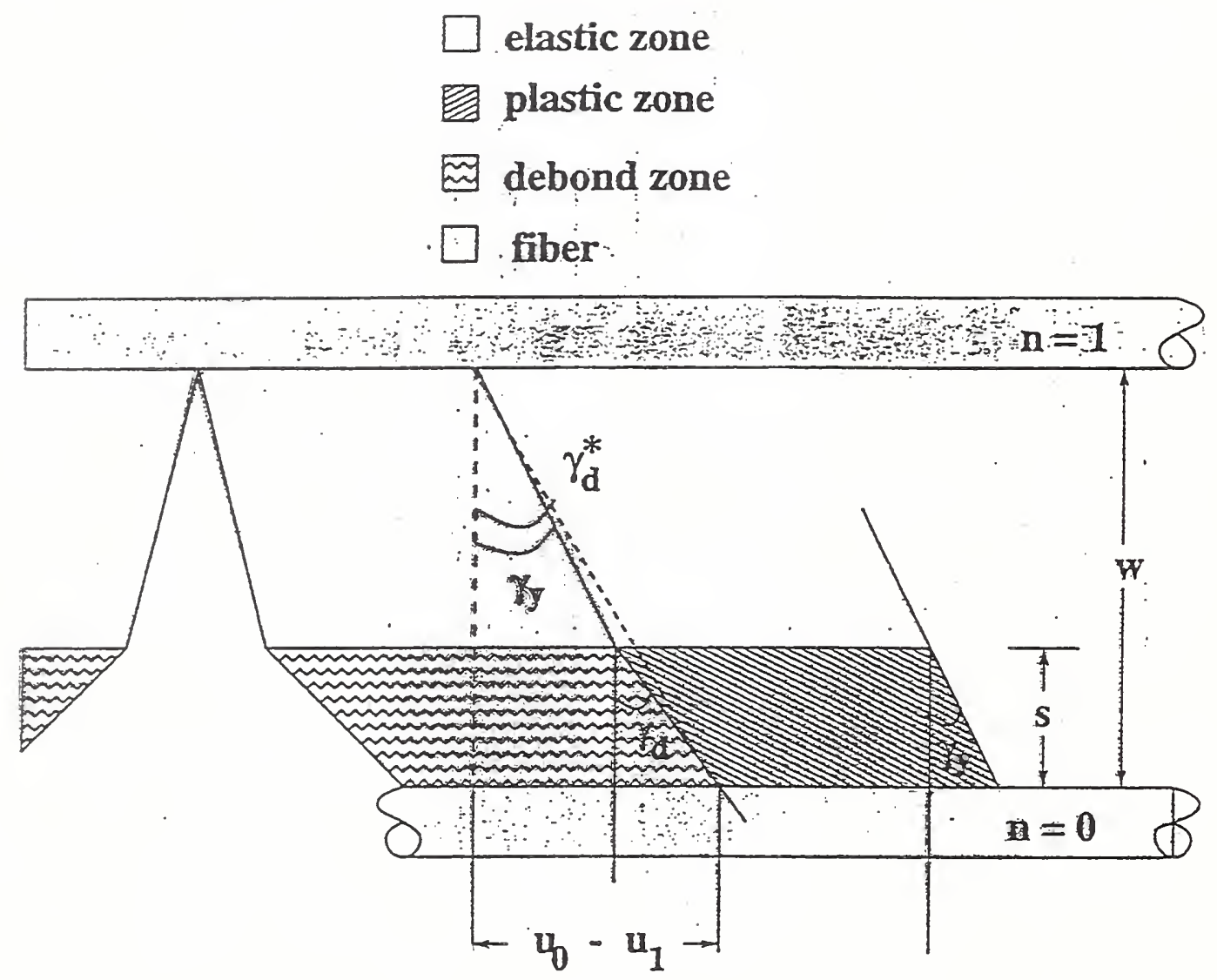

Transparency 非26 


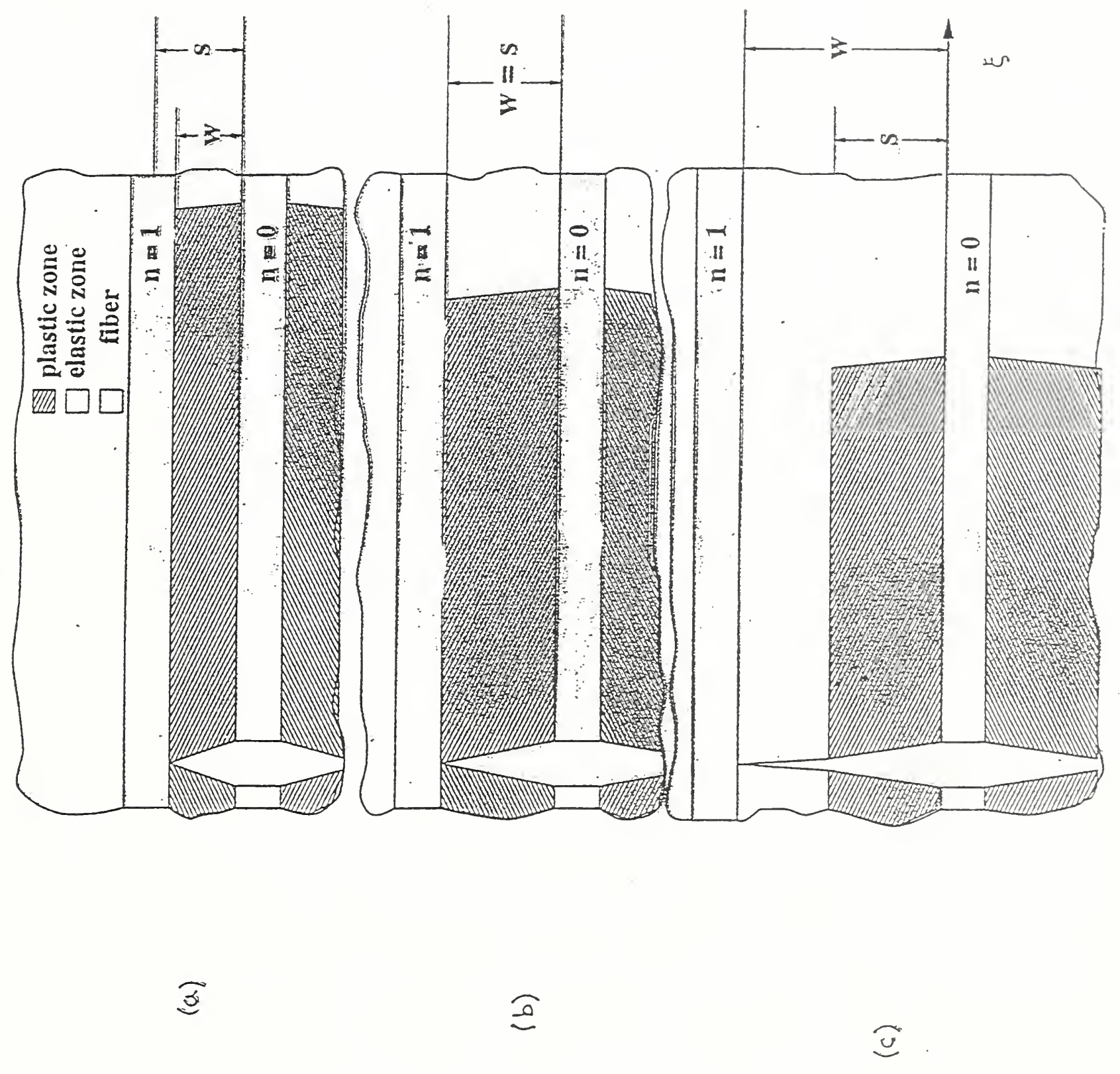

Transparency 非27 


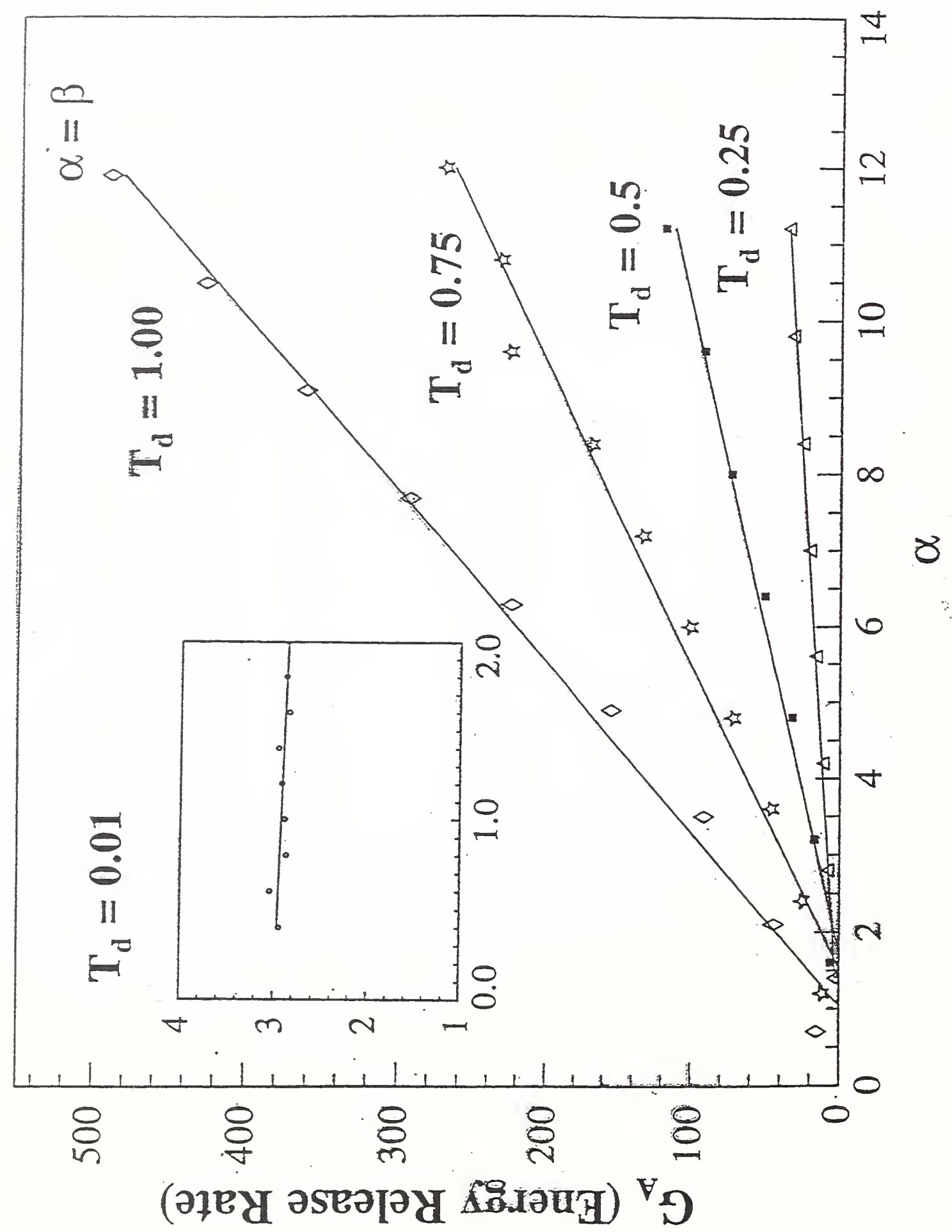

Transparency 非28 


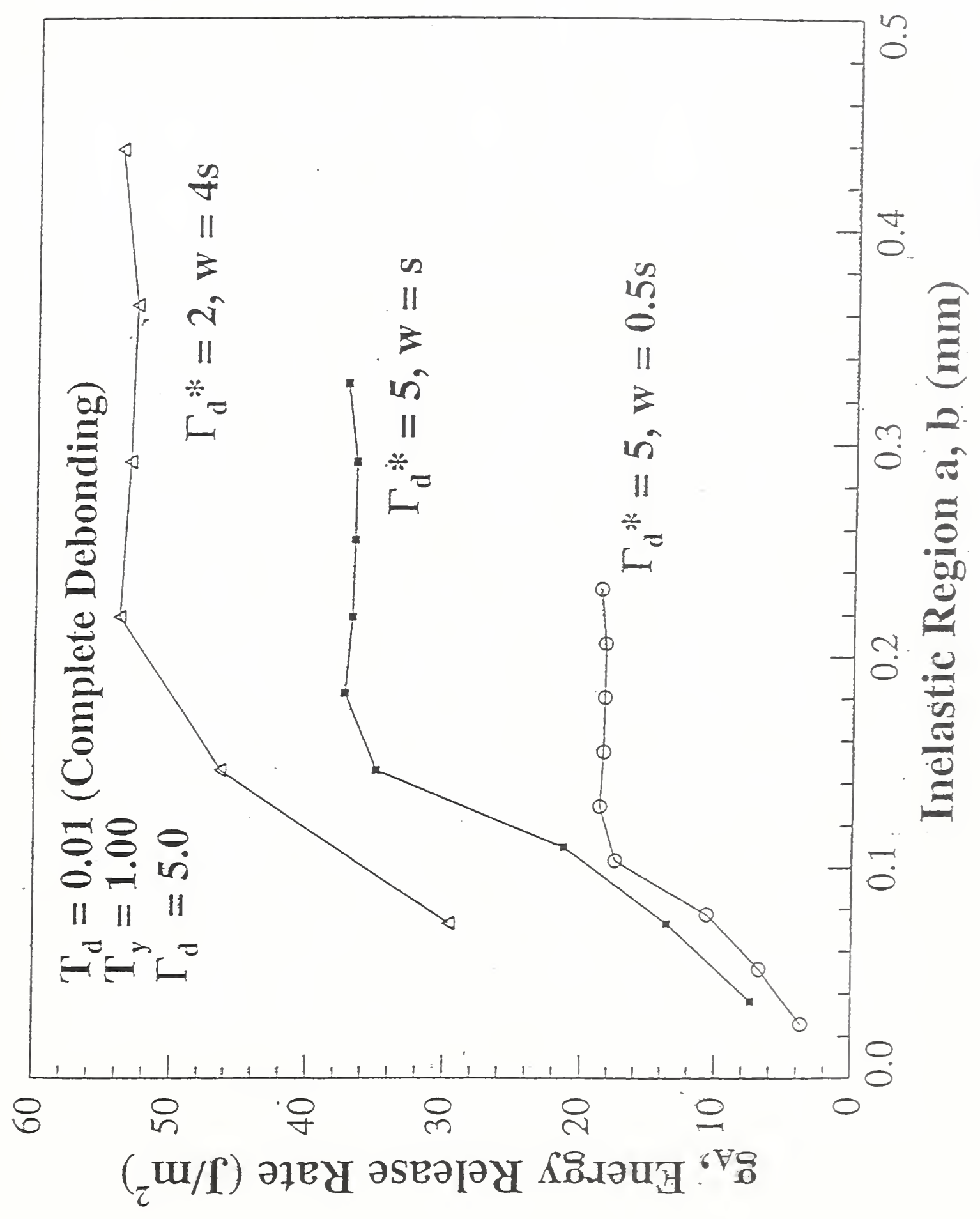

Transparency 非29 


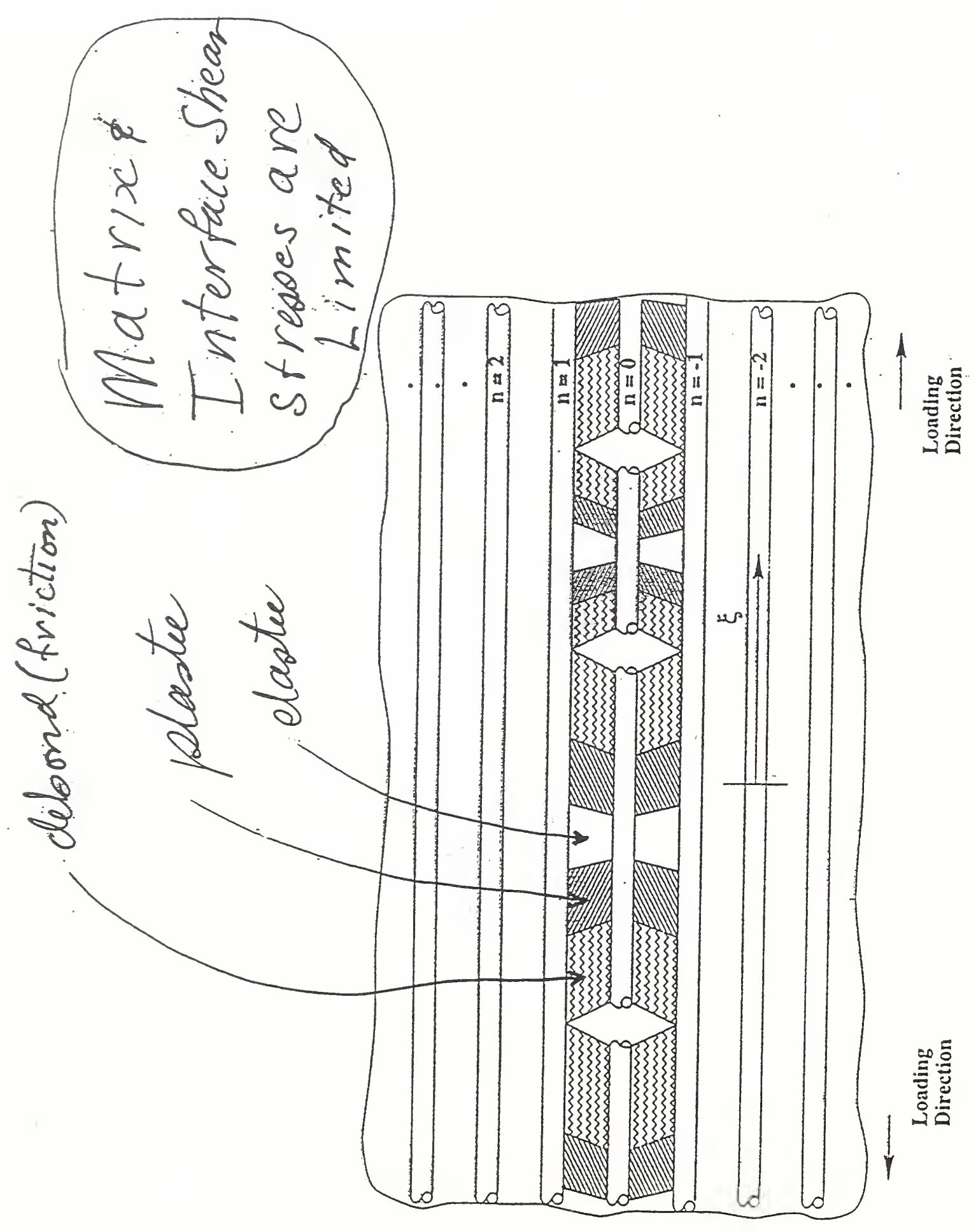

Transparency 非30 


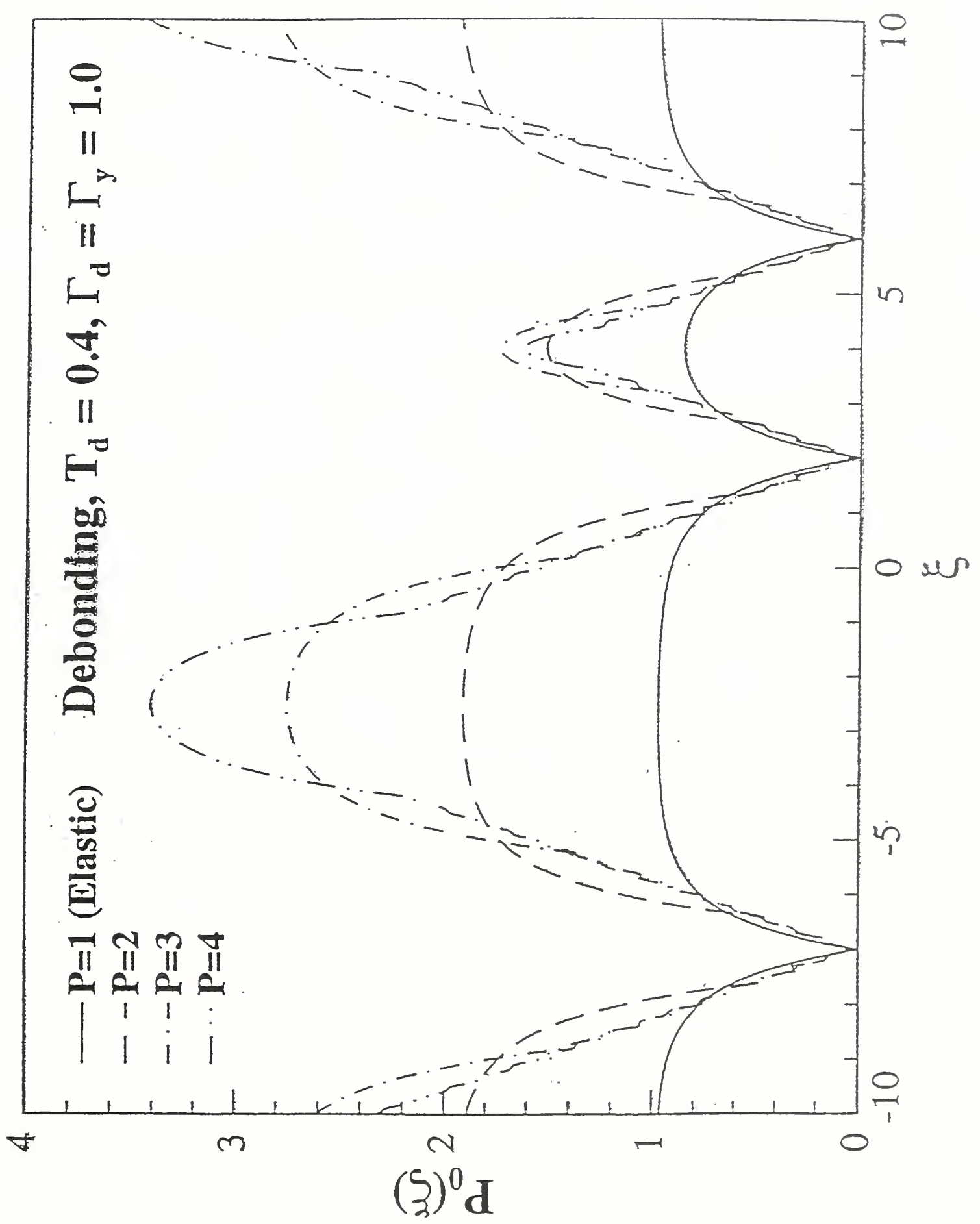

Transparency 非 1 
Comparison of the Interfacial Toughness Measured by the Single Fiber Pull-Out, the Indentation, and the Fragmentation Tests

\section{Dr. Andreas Hampe, Bundesanstalt fur Material Forshung und Prufung - BAM}

First I shall speak about the ductile - in this case, pure ductile - failure and then I shall discuss the brittle failure of the interface. For the single-fiber fragmentation, pull-out and indentation tests. I shall compare stress distributions, observations, and results. Before I start, some statements are needed: the aim of my talk is not to discuss methods to be used just for material comparisons, since every method will do that more or less. I want to discuss methods for the measurement of mechanical data. The situation with the micromechanical tests for interfaces is very complicated, consequently this talk will not give a complete picture but rather, a survey and then try to draw some guide lines.

We begin with the easier case to analyze, that is, the pure ductile failure of the interphase. This transparency shows the method of the classical fragmentation test, and in the ductile case, the expression for the interfacial shear strength in the Kelly-Tyson equation is correct because we have constant shear stresses at the interphase. We have a similar situation for the pull-out test. Here, we see the pull-out test as it is performed at our institute, but what I am saying in this talk about pull-out is valid for any kind of pull-out test, including the bead pull-off test. From the pull-out trace we get a maximum force and an embedded length and we are able to calculate, using the Kelly-Tyson formula, an interfacial shear strength since we have a ductile failure behavior. Cristian Marotzke has calculated the development of the shear stresses during this test, and here you see that we start at low loads with an inhomogeneous stress distribution with a high maximum near the matrix surface and a lower one near the fiber tip. For higher loads, yielding of the matrix produces a growing plateau region. This transparency shows you an example for such a ductile failure - a seldom seen case. We have measured the shear strength values for different embedded lengths and for quenched PET we find ductile behavior: no dependence from the embedded length in contrast to the slowly cooled material So we can make a first conclusion: in the ductile case, we can measure an interfacial shear strength with the fragmentation and the pull-out test.

Now I come to the brittle failure. Starting again with the fragmentation test, the stress distributions are now complicated: because of the brittle behavior of the matrix, we find a debonded zone with frictional stresses and a bonded region with a shear stress maximum at the crack tip. It is our opinion that we do not get enough information by performing this test, in particular, we do not get any information about friction, to evaluate a shear strength. Some groups have developed energy based evaluations. Daniel Wagner and John Nairn have proposed an energy balance: the difference of the strain energy before and after a fiber and interface crack should be equal the energy which is needed to create the fracture surfaces in the fibre and the interface. If the fibre fracture toughness is known, the toughness of the interface can be determined. For a glass fibre and an urethane-acrylate matrix they measured values from $183 \mathrm{~J} / \mathrm{m}^{2}$ for a weak and $264 \mathrm{~J} / \mathrm{m}^{2}$ for a strong interface. But a question is whether this energy balance equation is correct, that is, are all of the energies considered. I think that this is a principle problem of the micro mechanical tests, where the initiation, the creation of cracks is included in the data reduction scheme: you have to take into account additional losses, but you don't know what they are.

The method of Pegoretti, Accorsi and DiBenedetto avoids this problem. They are calculating, by 
finite element analysis, the $G_{c}$-values as a function of the different cracks: the interface, the shear, and transverse matrix crack. They argue that the crack arrests at that $\mathrm{G}_{\mathrm{c}}$ value which corresponds to this matrix stress and this crack length. The results for different samples are interfacial fracture toughnesses from 60 to $340 \mathrm{~J} / \mathrm{m}^{2}$. In addition to this work, Lars Berglund is also investigating growing cracks by a finite element simulation. For carbon fibers and epoxy matrix, he found interfacial fracture toughnesses of $200 \mathrm{~J} / \mathrm{m}^{2}$ for a weak and $240 \mathrm{~J} / \mathrm{m}^{2}$ for a strong interface.

One big uncertainty of these results is that we have no information of the effect of friction. You can see in the transparency that the calculated energy release rate in the fragmentation test versus the crack length for $\mu$ equal to zero and $\mu$ equal to one. The energy release rate with large friction is about five times smaller than the energy release rate without friction. This leads to an overestimation of $\mathrm{G}_{\omega}$ if you don't take friction into account when using the fragmentation test. It is a disadvantage of the fragmentation test, that only two characteristics, the matrix stress and the crack length, are available.

This situations changes totally, when we look at the single fibre pull-out test. Let us look again at the stress distributions. We have to differentiate two cases, the long and the short embedded fibre. This has some similarities with the fragmentation test, but in the following I am speaking about the long embedded fibers: as in the fragmentation test, the shear stress has a constant friction value in the debonded region and this complex distribution in the bonded region has two maxima. The axial normal stresses in the fibre are increasing from the fibre tip to the matrix surface. We are performing the tests in very stiff machines, using piezo translators and sensors. For the system glass fibre and polystyrene matrix, we have measured this force displacement trace with two slopes in the rising part. As in the fragmentation test, we have the possibility to observe the interface during the experiment, but here we know where we have to look. So, in addition to the trace we get these correlated pictures of the crack (fiber diameter is $18 \mu \mathrm{m}$ ). The black stripes indicate the matrix surface, and the green dots indicate the fiber tips.

We recognize that the crack is growing in the second part of the trace, the part with the smaller slope. In this slide, the crack lengths are plotted as red circles. We started with a constant small crack, here the crack is growing and also plotted is the embedded length $\mathrm{L}_{\mathrm{e}}$ with about $270 \mu \mathrm{m} . \mathrm{L}_{\mathrm{e}}$ is correlated to this friction force because here we have a totally debonded fiber totally sticking in the matrix. From this pull-out force and the embedded length we can calculate the frictional shear stress. With the frictional shear stress, we can calculate the friction forces for all crack lengths, the forces which are necessary to overcome friction. If we subtract this force from the measured force, we get the force at the crack tip.

The crack driving forces are plotted in this slide as squares. The force at the crack tip is nearly constant, only for larger crack length we find a slight decay. This corresponds to the stress situations at the crack tips: the calculated stress intensities are nearly constant for a long range and than rise. These are calculated for constant loads. If we assume the failure at constant stress intensities, then the measured load has to decrease. We calculated the fracture toughness of the interface by using these data and we got values for this example from 3 to $6 \mathrm{~J} / \mathrm{m}^{2}$. Now, it seems that this is a nice picture which describes the experiment completely and in accordance to theory. But there is still an uncertainty: the influence of residual stresses. When we cool down the melted matrix with the 
embedded fibre, we are producing stresses in our sample. You can see the half globe of matrix, fiber, and aluminum bar. On the left side is a finite element net, on the right side are the stresses experienced. The comparison of the energy release rate of a system with a thermal load of $130{ }^{\circ} \mathrm{C}$ with a system without a load indicates that by a factor of two to three the loaded system releases more energy than the unloaded. This we have to take into account.

For the indentation test, the stress distribution shows the same characteristics as that from the pull-out for the long fibre, but with a negative sign. I skip the stress-based method and come to the energybased method. When we are increasing the load, we get a growing crack. We measure force and displacement. Marshall and Oliver have proposed a data reduction scheme for the determination of the frictional stress and the debonding energy for ceramic matrices. Therefore, they plotted the indenter displacement versus the square of the force. The determination of the slope $\mathrm{m}$ and $\mathrm{u}$-o leads to $\tau$ friction and the debonding energy. Gerd Kalinka, a colleague in our group, has varied this method to some extend in order to use it for polymeric matrices. He has designed a compact, stiff and inexpensive machine. Recently I have examined his data reduction scheme by the measured pull-out traces. If we plot all the stress distributions on one transparency, we see that for the pull-out and the indentation the distributions are mirrored, as I just mentioned. I found the same for PS and PC, this force displacement trace for a glass fibre pulled out of a PUA sample, for which we measured $G_{c}$ values about $20 \mathrm{~J} / \mathrm{m}^{2}$ in the reduced displacement versus force square trace. The result is that we can easily determine the slope and $u-o$ and get a result for $G_{c}$ which is much smaller than the $20 \mathrm{~J} / \mathrm{m}^{2}$. Consequently, we have to improve the theory.

Now I come to the conclusions: if we compare the methods in order to get these measurements we have to realize that there are large differences in the availability of data: the most difficult situation has the fragmentation test with just two numbers, the most comfortable situation is the pull-out test with force displacement trace including a frictional part. The measured $G_{c}$ data I reported are compared in this transparency. The effect of residual stresses is not regarded. We have to recognize that we can compare only one material: for the system glass fiber and PUA we find enormous differences. But the consideration of additional losses, especially friction, would lead to lower values here and the consideration of residual stresses would lead to higher values for the pull-out test.

The main massage of the next slide is that we need more measurements for the same systems. In my last transparency I have tried to easy the comparison of the three methods by this matrix. Fragmentation, pull-out, and indentation tests are compared. In the fields are information on which data with which error can be measured, and which problems and which limitations we are still facing. Ductile failure is easy: fragmentation and pull-out work well, result is $\tau_{\text {interface }}$. In the brittle case, the experiments which include the initiation of a crack have the disadvantage of larger additional losses which are unknown but influence the result. In the case of crack propagating experiments, I think we are allowed to assume that the influence of such additional effects can be neglected. For the indentation test, we have to improve the data reduction scheme. So these two cases remain: the crack propagating experiment with the fragmentation and the pull-out method with advantages for the pullout: we get more information, especially the information about friction and we are not restricted to matrices with a strain larger than the strain of the fiber. My conclusion is that if we intend to measure mechanical characteristics of the interface, the pull-out method is a good choice. 


\title{
Comparison of the Interfacial Toughness Measured by the Single Fibre Pull-Out, the Indentation, and the Fragmentation Test
}

\author{
A. Hampe, G. Kalinka, C. Marotzke \\ Bundesanstalt für Materialforschung und -prüfung, BAM \\ (Federal Institute for Material Research and Testing) \\ Berlin, Germany
}

\section{Objectives}

Ductile failure of the interphase Fragmentation test Pull-out test

Brittle failure of the interface

Fragmentation test

Pull-out test

Indentation test

Comparison of stress distributions

observables

(characteristics)

results

Conclusion 


\section{Preliminary remarks:}

Aims: Methods not just for material comparison but for measurement of mechanical data

Not results for specific materials but the possibilities and the limitations of methods

The situation is complicated: we have three test families, each with many members

Consequence: the talk gives not a complete picture but tries to draw simple guide lines 
Ductile Failure of the Interphase

Fragmentation Test

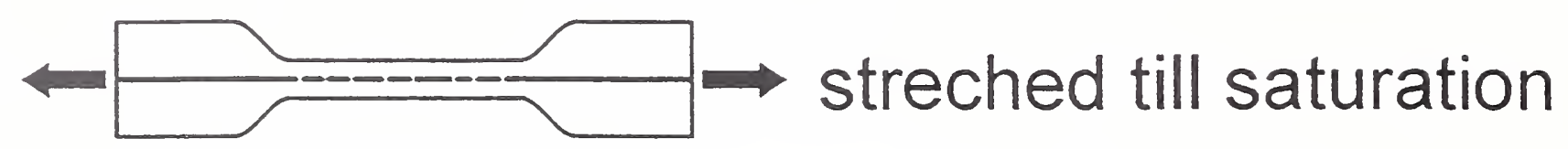

Measurement of

Fragment Length
\[ L \text { Frag } \]

+ Fibre Strength at Fragment
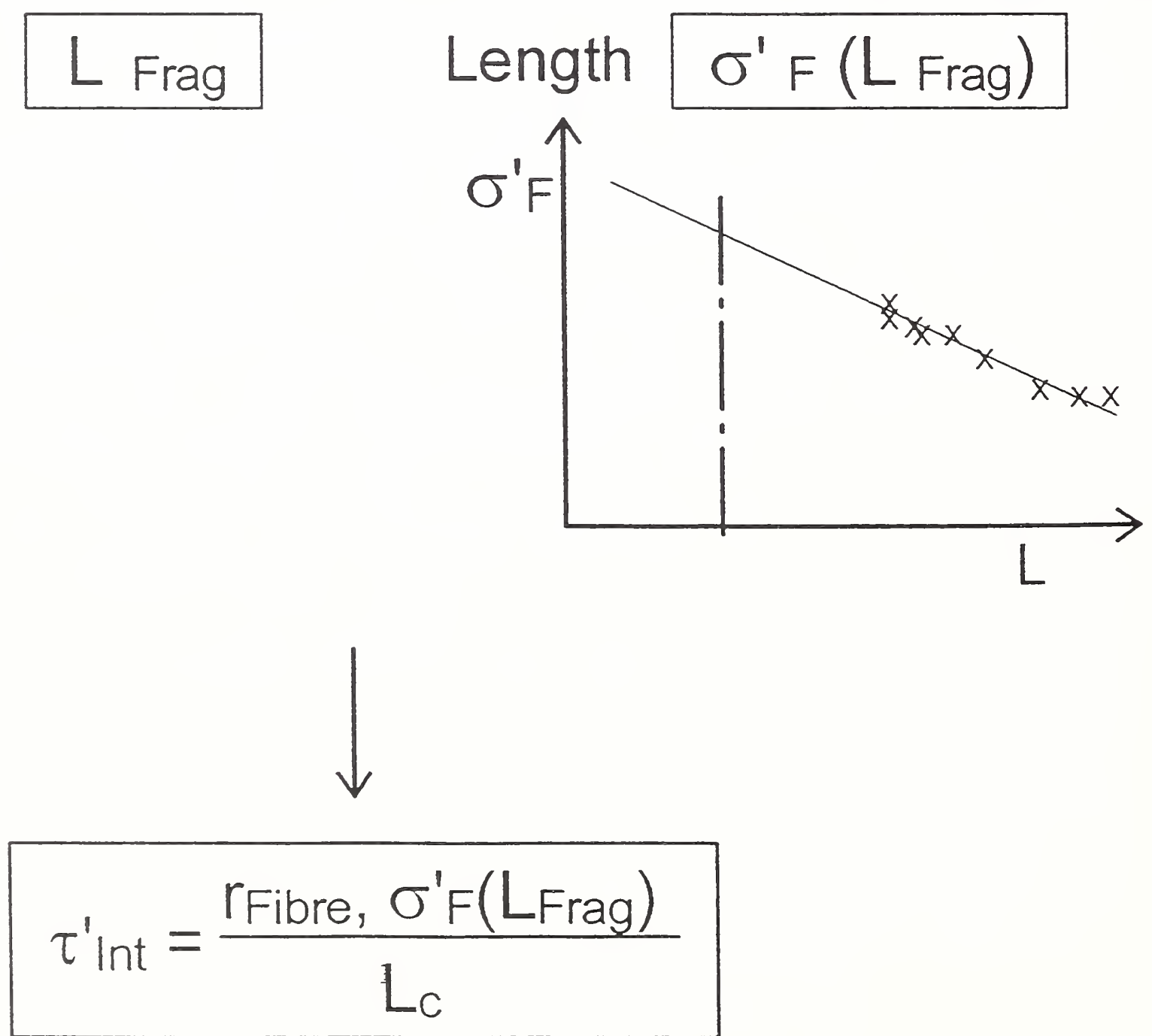
Ductile Failure of the Interface

Stress Distribution

Fragmentation Test

ofbre, Tinterface

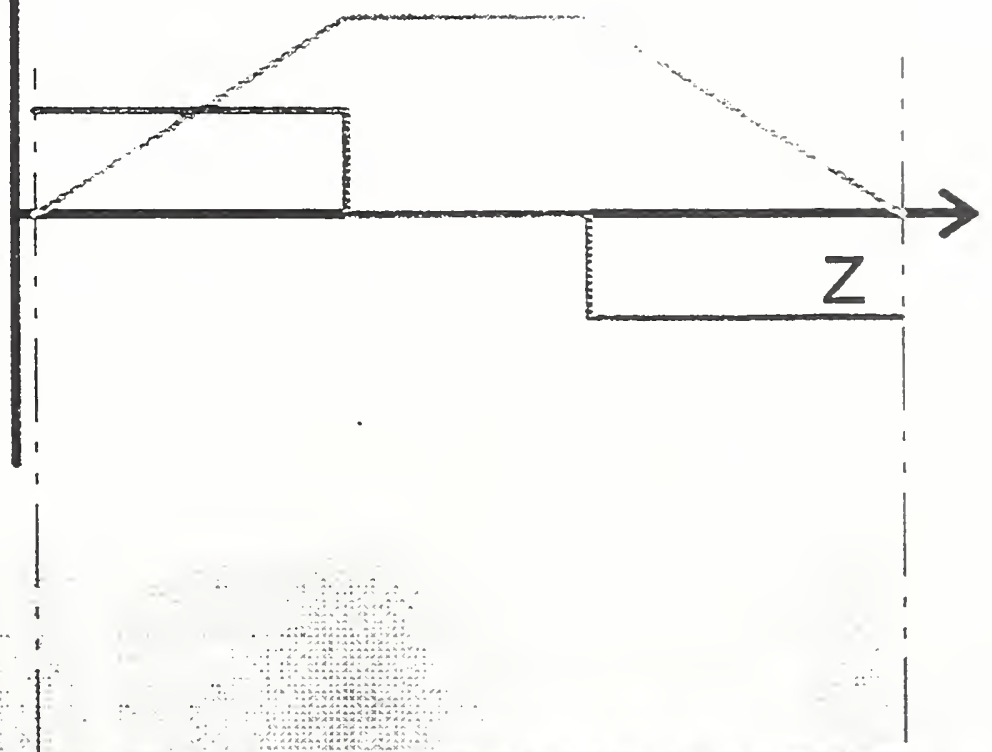


Ductile Failure of the Interphase

Pull-Out Test
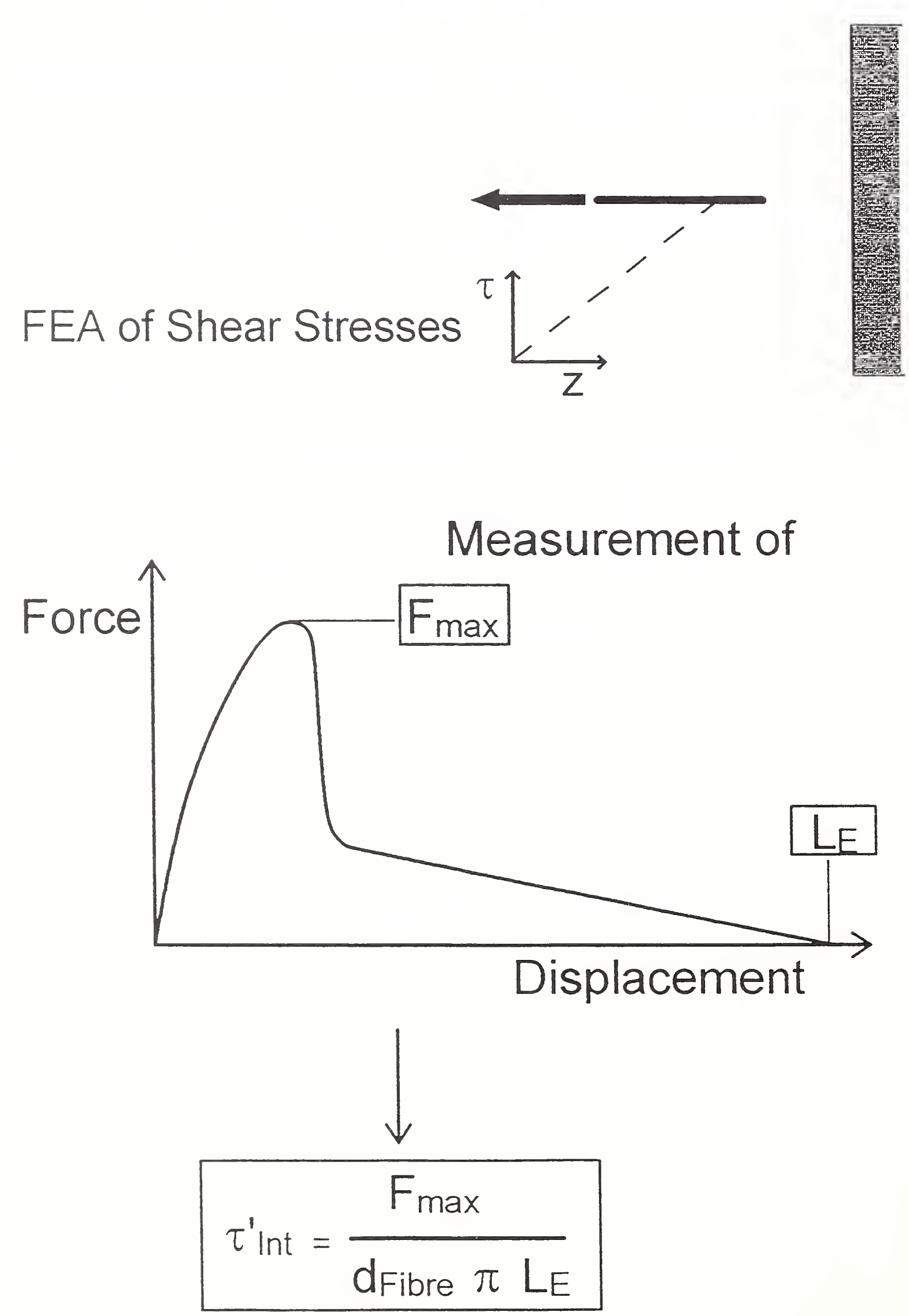


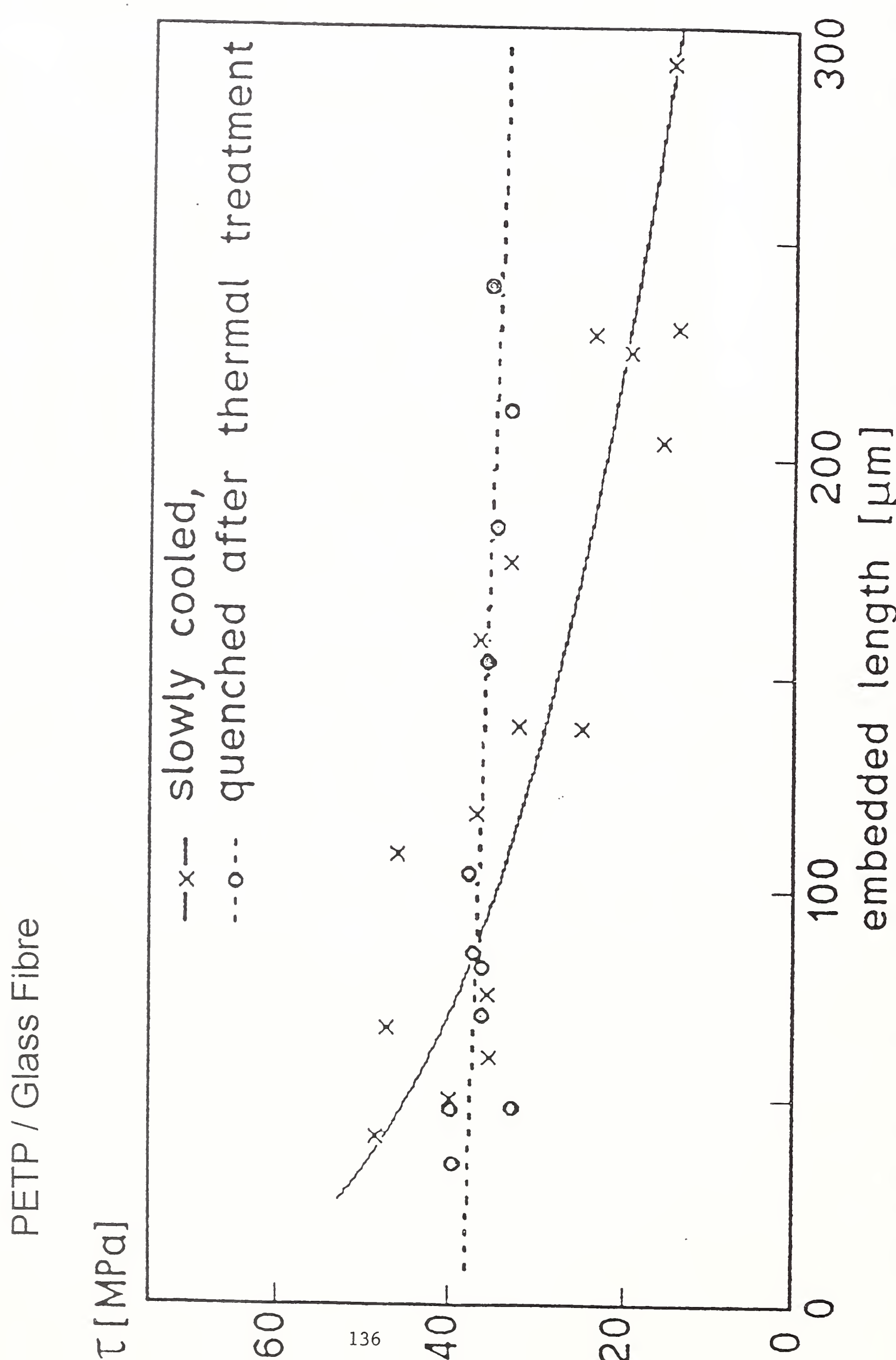


Ductile Failure of the Interface

Conclusion

Fragmentation Test

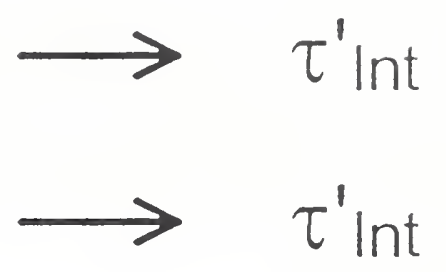

Indentation Test

? 


\section{Brittle Failure of the Interface}

Stress Distribution

Fragmentation Test

Gofre, Tinterface

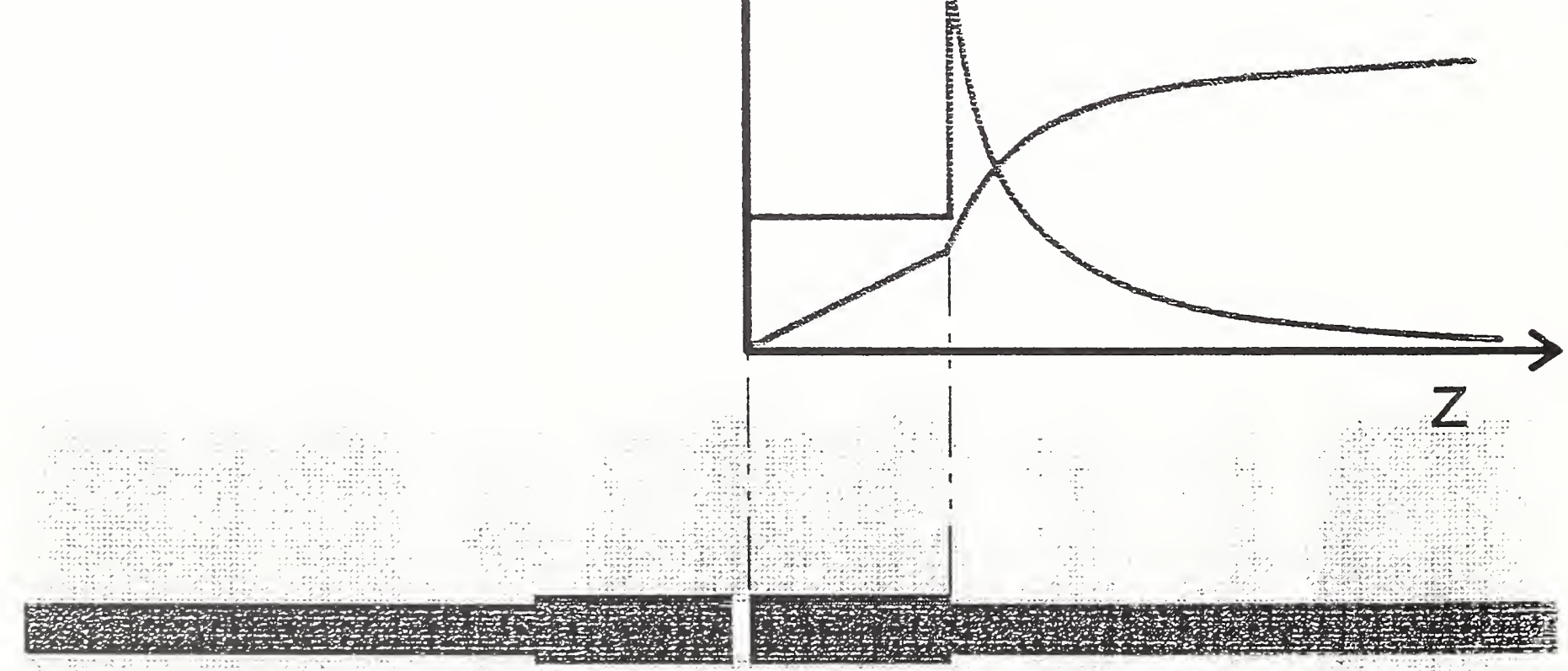




\section{Brittle Failure of the Interface}

Fragmentation Test

\section{Energy based method: Wagner, Nairn}

\section{Measurement of}

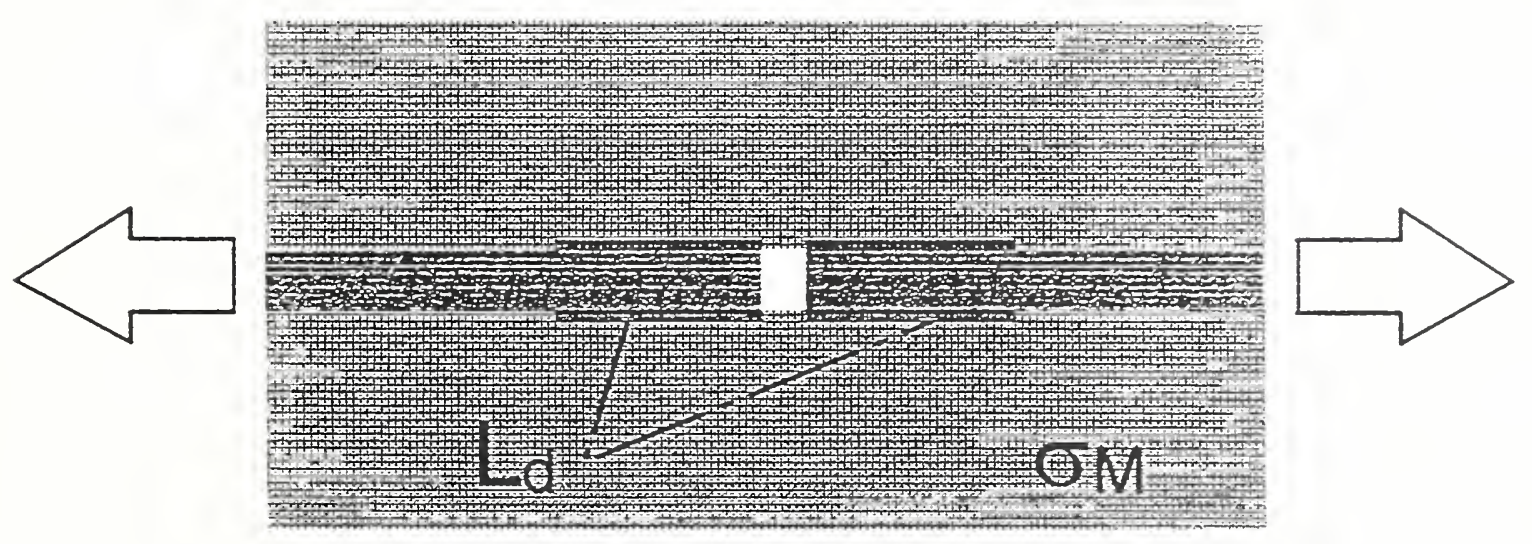

\section{Calculation of $\Gamma_{\mathrm{i}}$ :}

Energy available $=$ Energy to form fracture surfaces

$\left(\bigcup_{f}^{\text {prior }}+U_{m}{ }^{\text {prior }}\right)-\left(\bigcup_{f}^{\text {post }}+U_{m}{ }^{\text {post }}\right)=2 \pi r_{f} L_{d} \Gamma_{i}+\pi r_{f}^{2} \Gamma_{f}$

Result for glass fibre / polyurthane-acrylate

$$
\Gamma_{i, w}=183 \mathrm{~J} / \mathrm{m}^{2} \quad \Gamma_{\mathrm{i}, \mathrm{s}}=264 \mathrm{~J} / \mathrm{m}^{2}
$$


John Nairn:

"... energy does not control fibre fracture; instead, fibre fracture releases much more energy than required"

Excess released energy leads to additional energy dissipation by:

interfacial debonding matrix cracks

friction

plastic deformation sound emmission

? 
Brittle Failure of the Interface

Fragmentation Test

Energy based method: Dibenedetto et al.

Measurement of

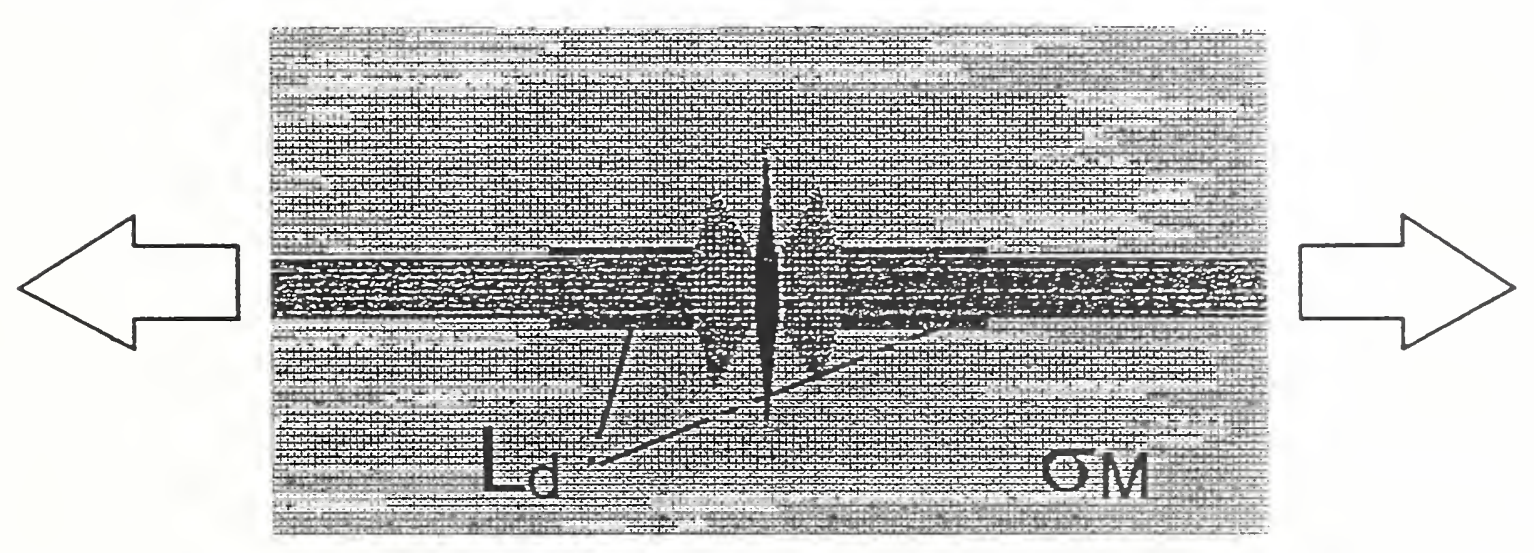

Calculation of

Strain energy release rate $G=f\left(\right.$ Crack length $\left.L_{d}\right)$

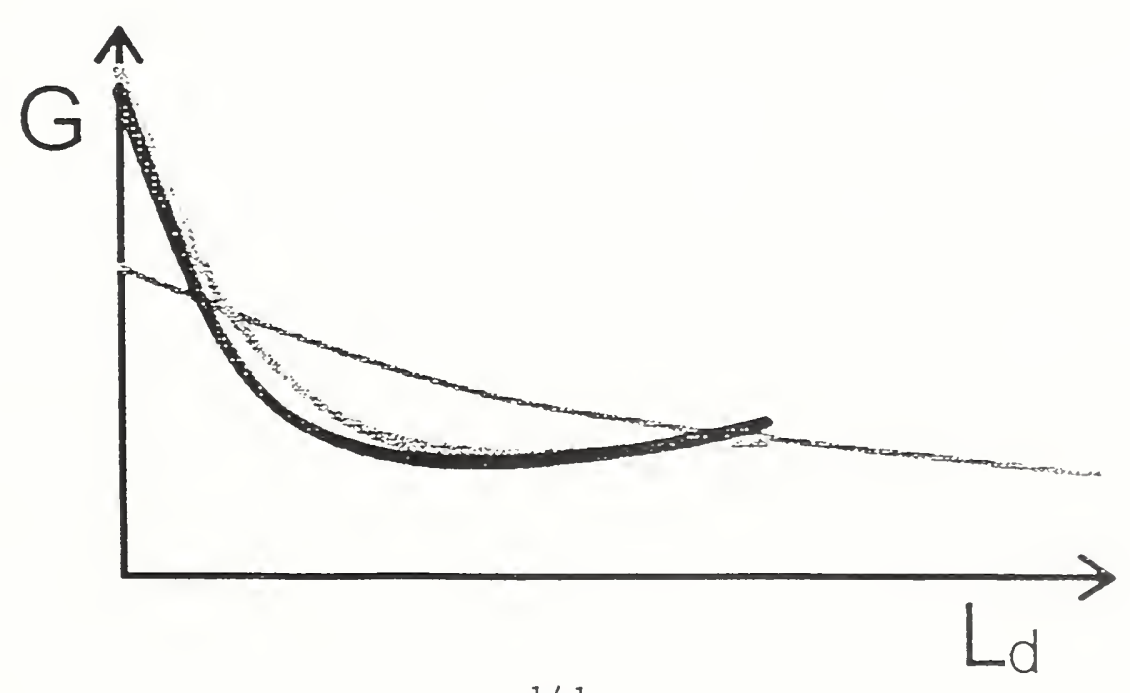




\section{Brittle Failure of the Interface}

Fragmentation Test

\section{Energy based method: Berglund}

\section{Measurement of}

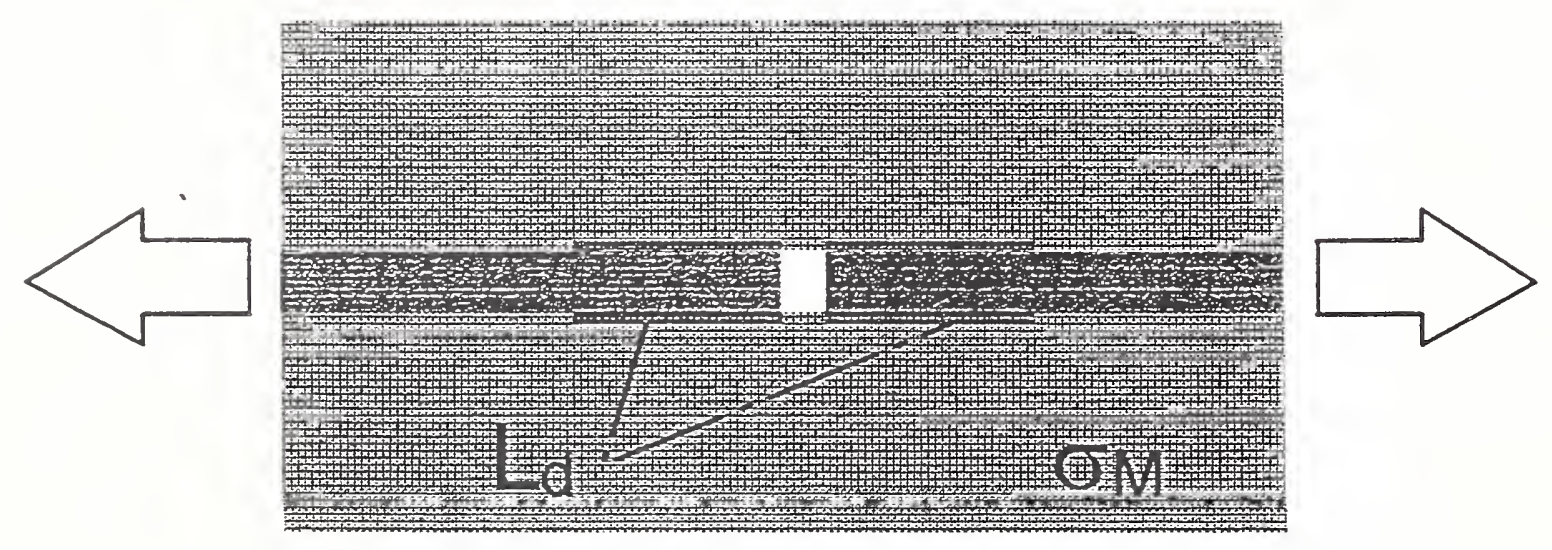

\section{Calculation of $G_{c}$}

by FEA with the failure criterion $-d U / d a=2 \pi r_{f} G_{c}$

Result for HTA carbon fibre / epoxy

diglycidyl ether of bisphenol A / diethylene amine

$$
G_{c, w}=200 \mathrm{~J} / \mathrm{m}^{2} \quad G_{c, s}=240 \mathrm{~J} / \mathrm{m}^{2}
$$




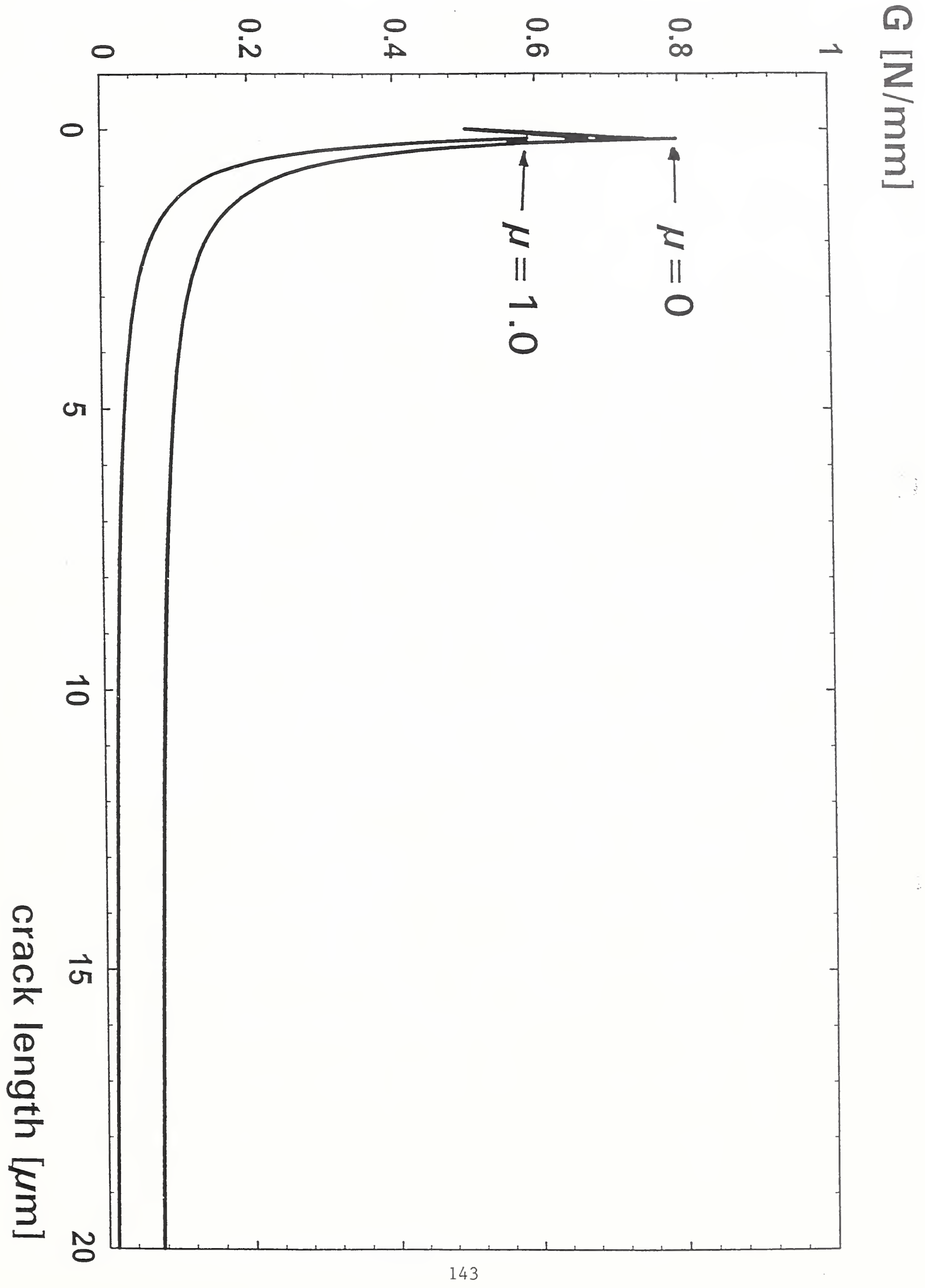




\section{Brittle Failure of the Interface}

\section{Stress Distribution}

Pull-Out Test

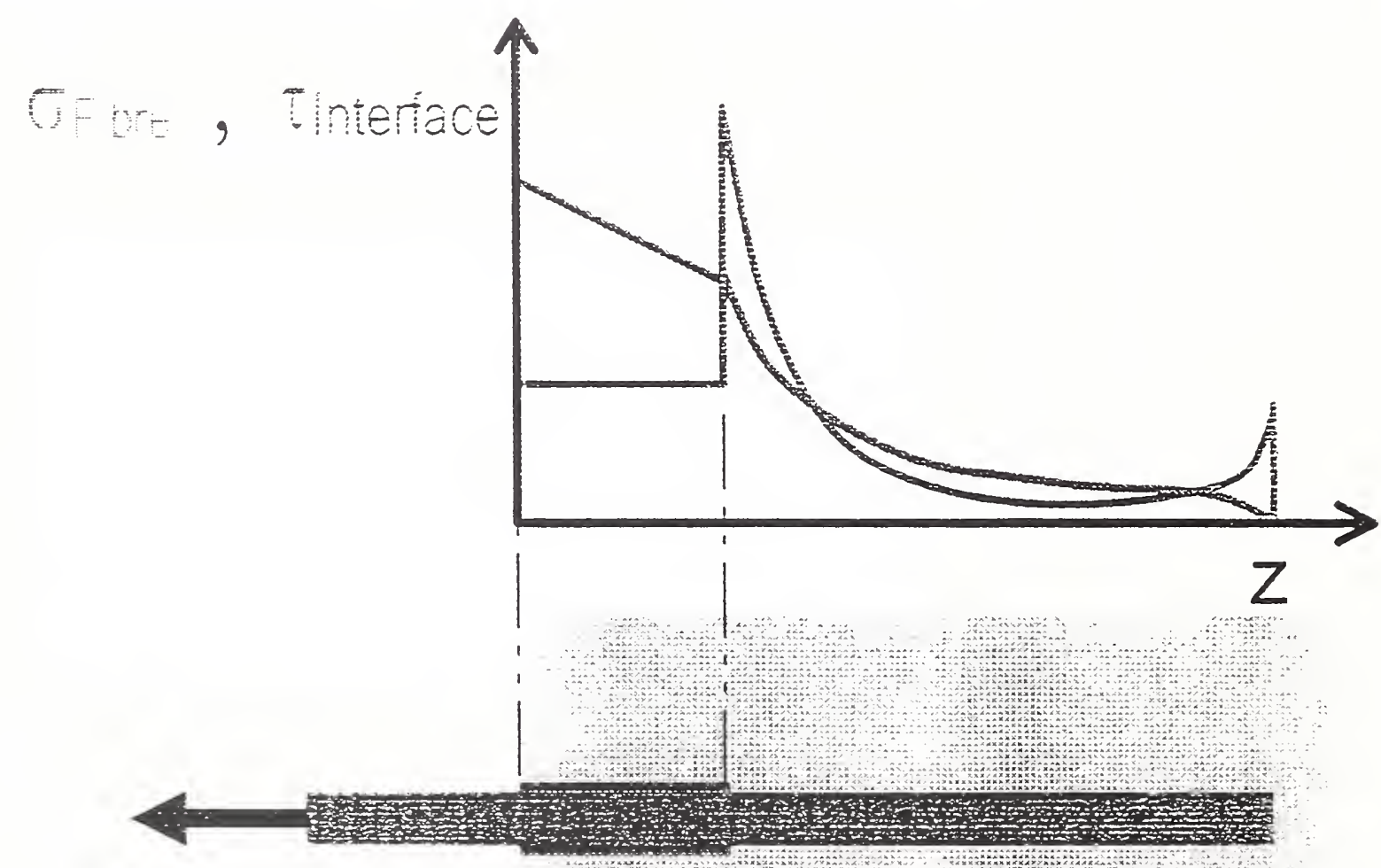

Oriore, Tinteriace

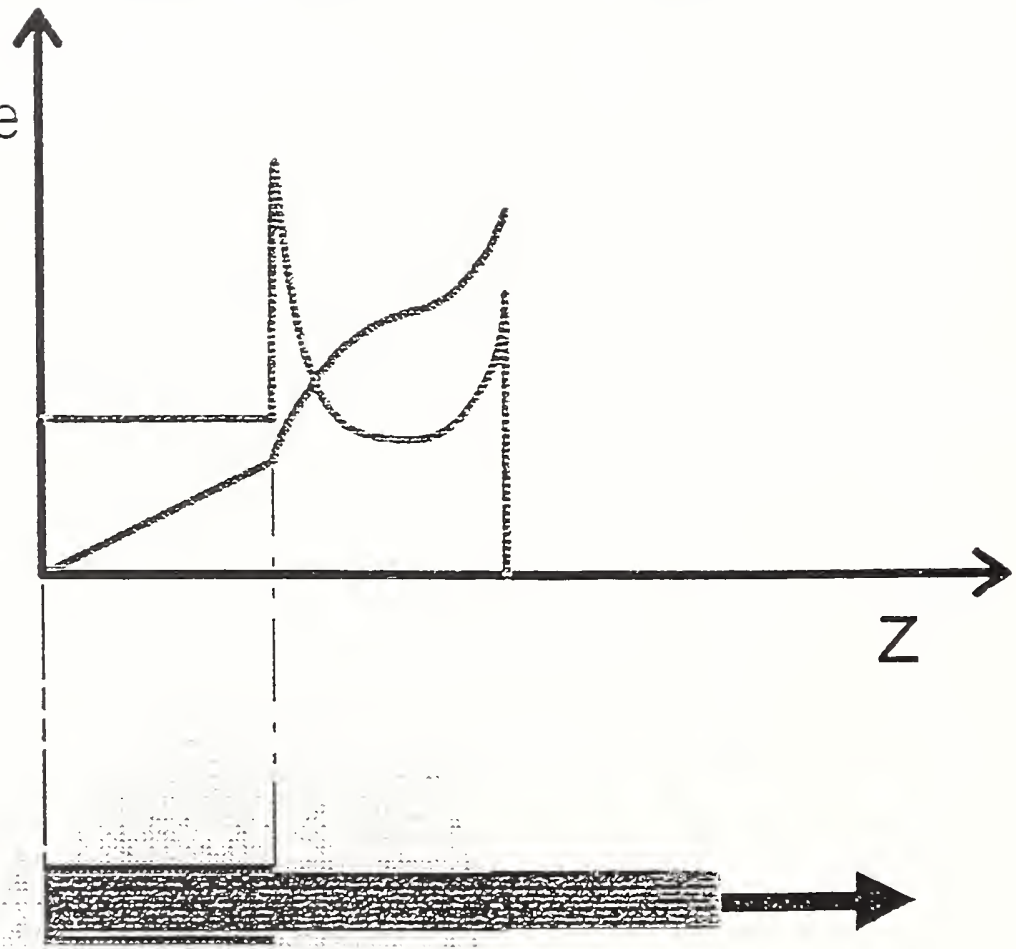


piezo force sensor

piezo translator $0 . .180 \mu \mathrm{m}$

matrix droplet

free fibre

$0 . .200 \mathrm{mN}$

steel frame

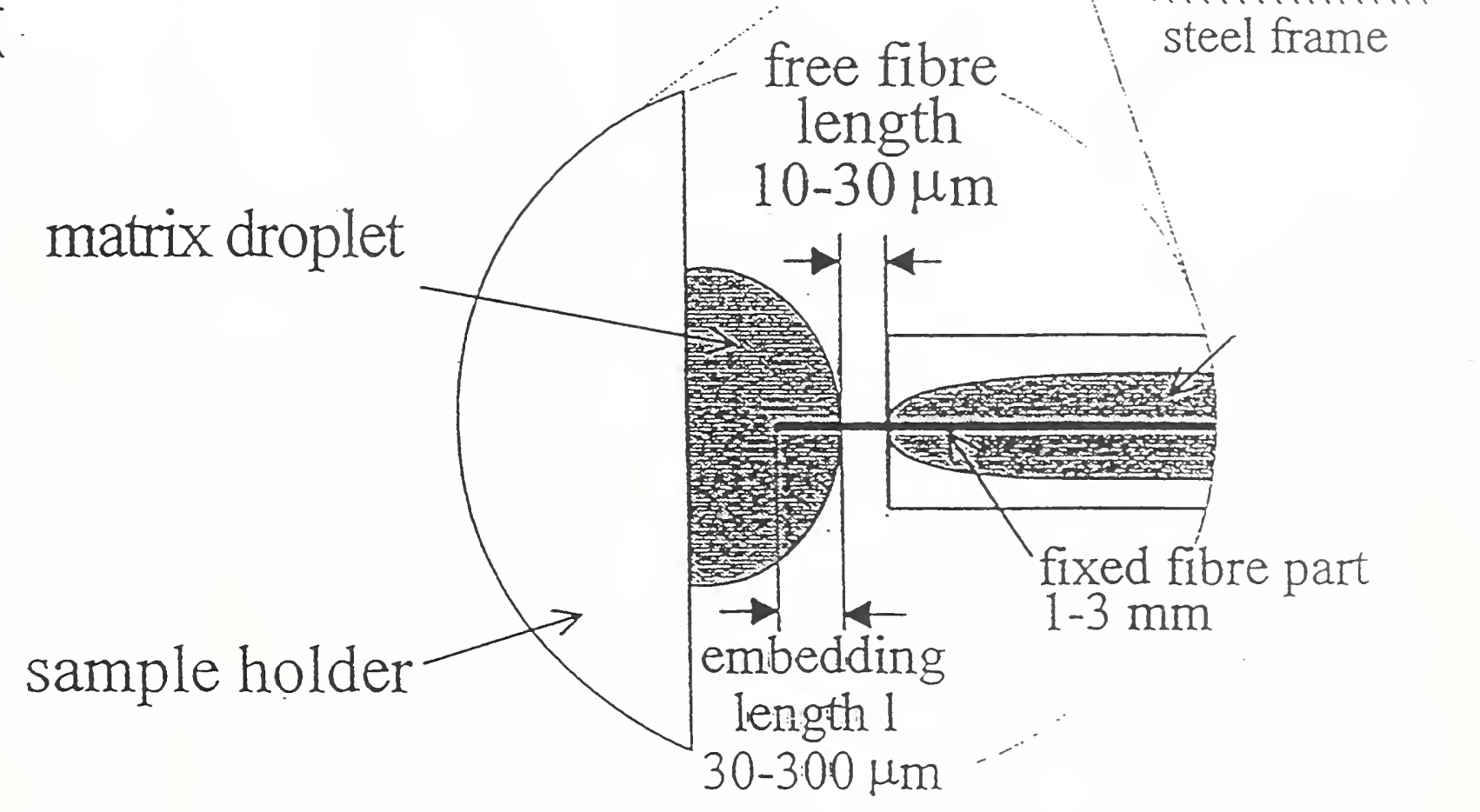

Stiffness of translator

Stiffness of load cell

Stiffness of free fibre length

$$
\mathrm{I}=30 \mu \mathrm{m}, \mathrm{r}=5 \mu \mathrm{m} \quad 0,2 \mathrm{~N} / \mu \mathrm{m}
$$

Stiffness of frame
$10 \mathrm{~N} / \mu \mathrm{m}$

$4 \mathrm{~N} / \mu \mathrm{m}$

$1 \mathrm{~N} / \mu \mathrm{m}$ 


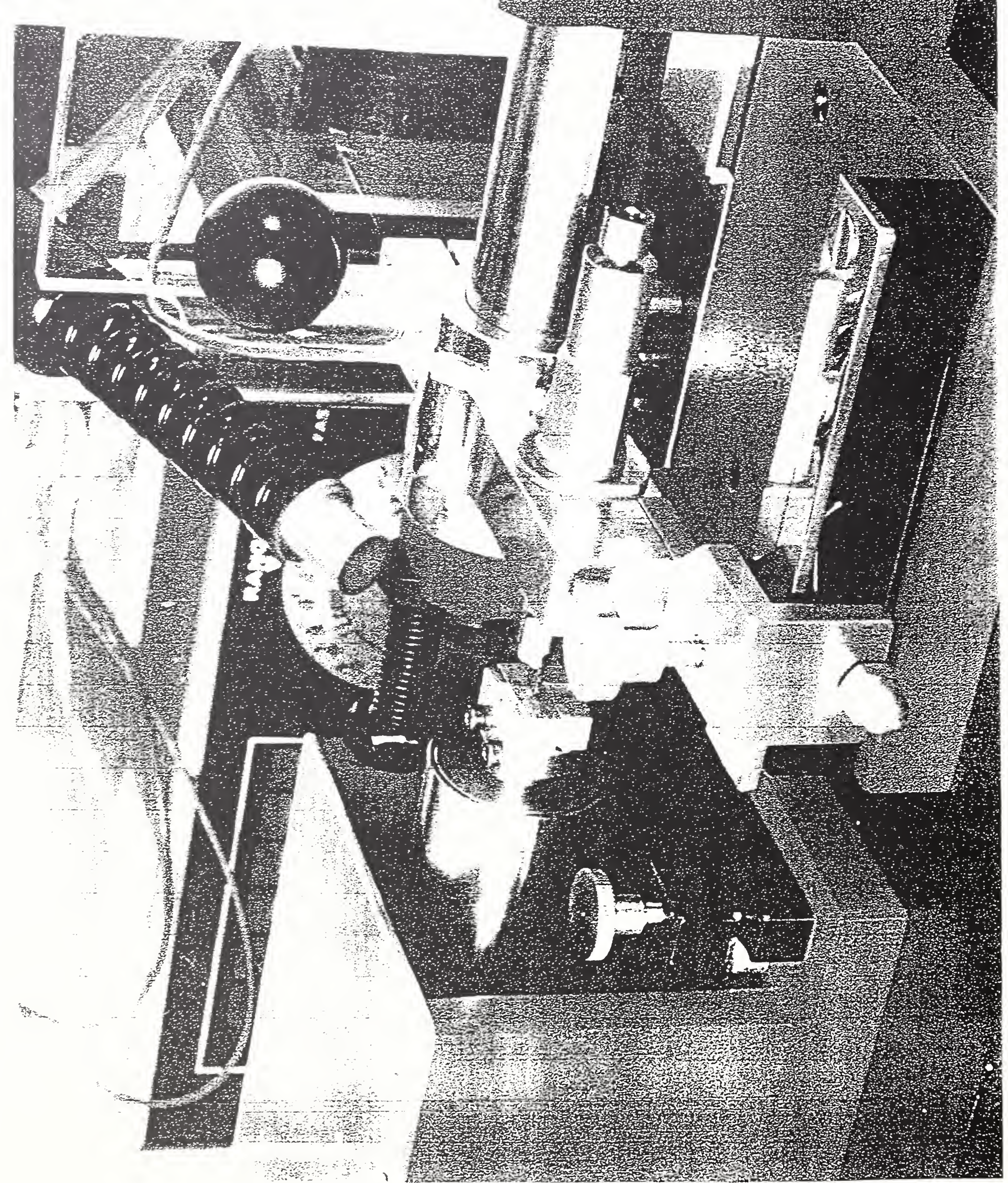




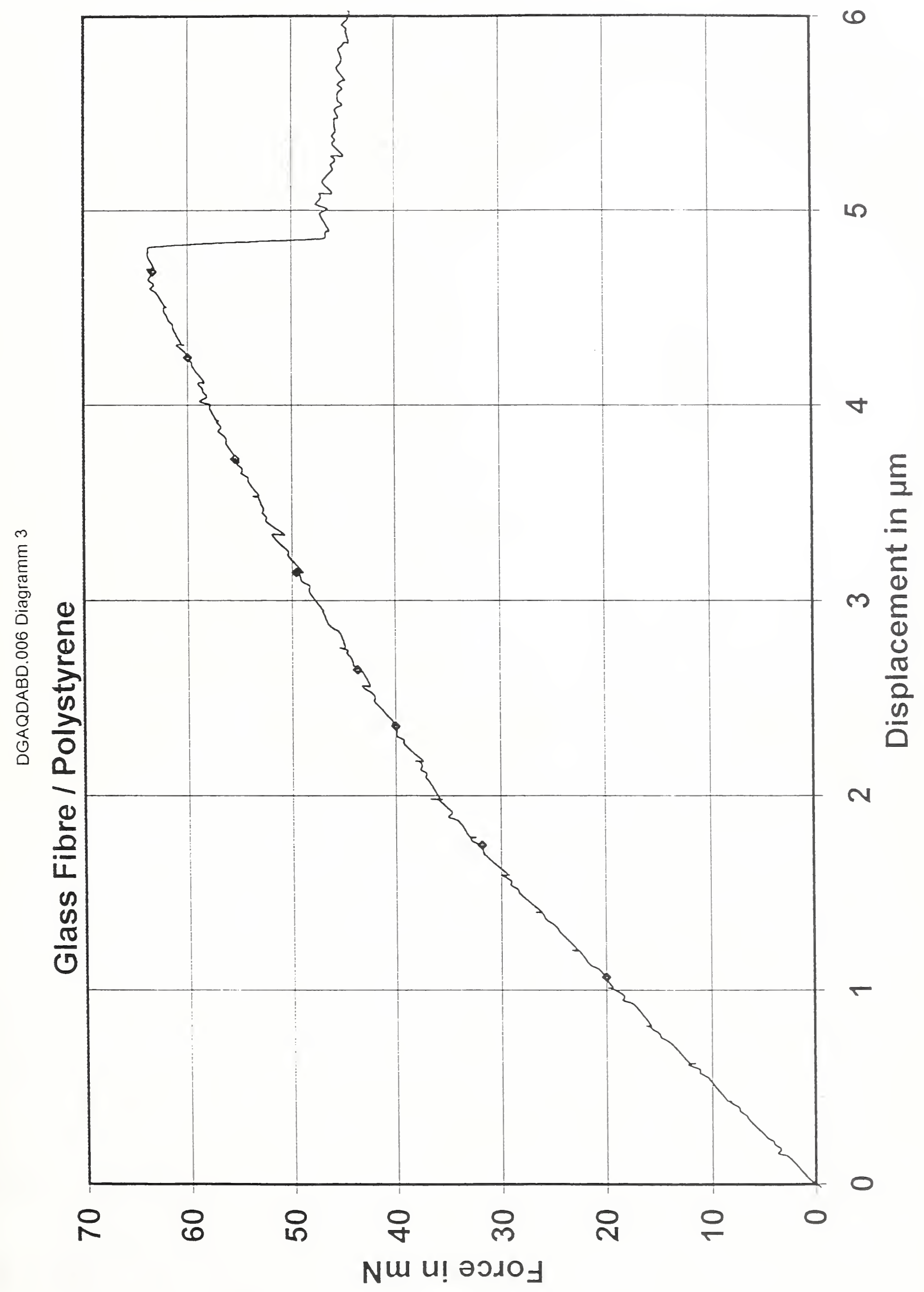




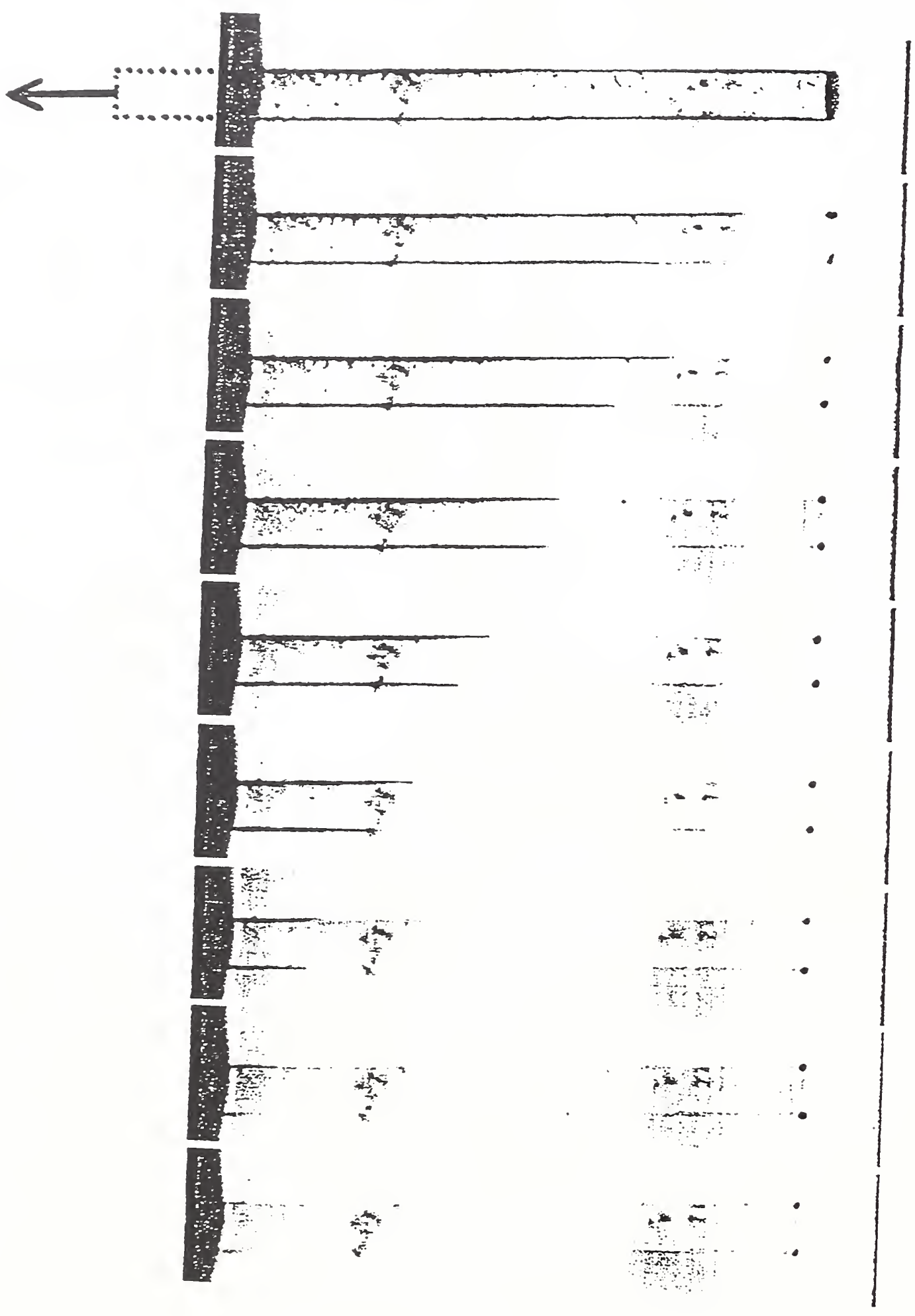




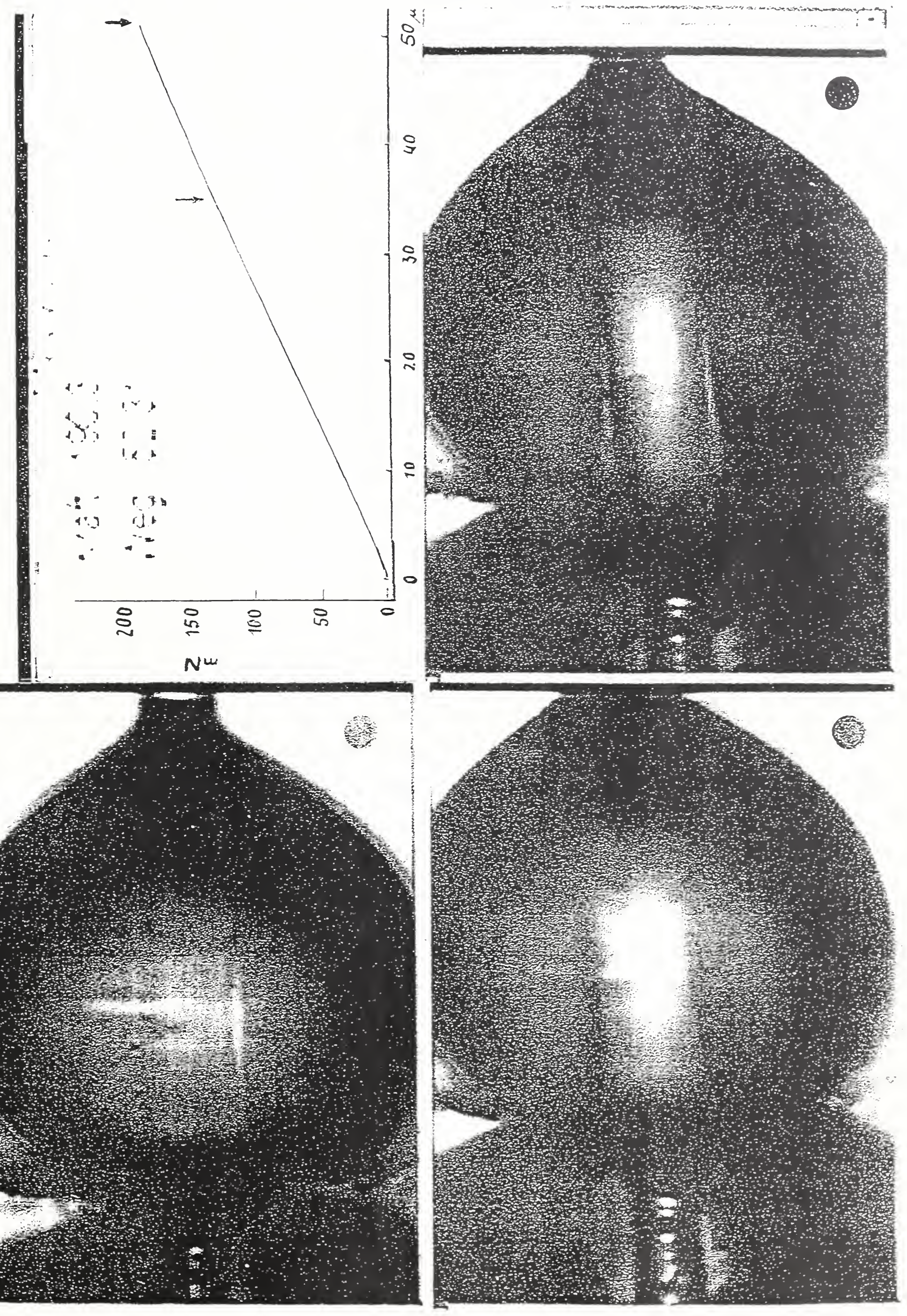



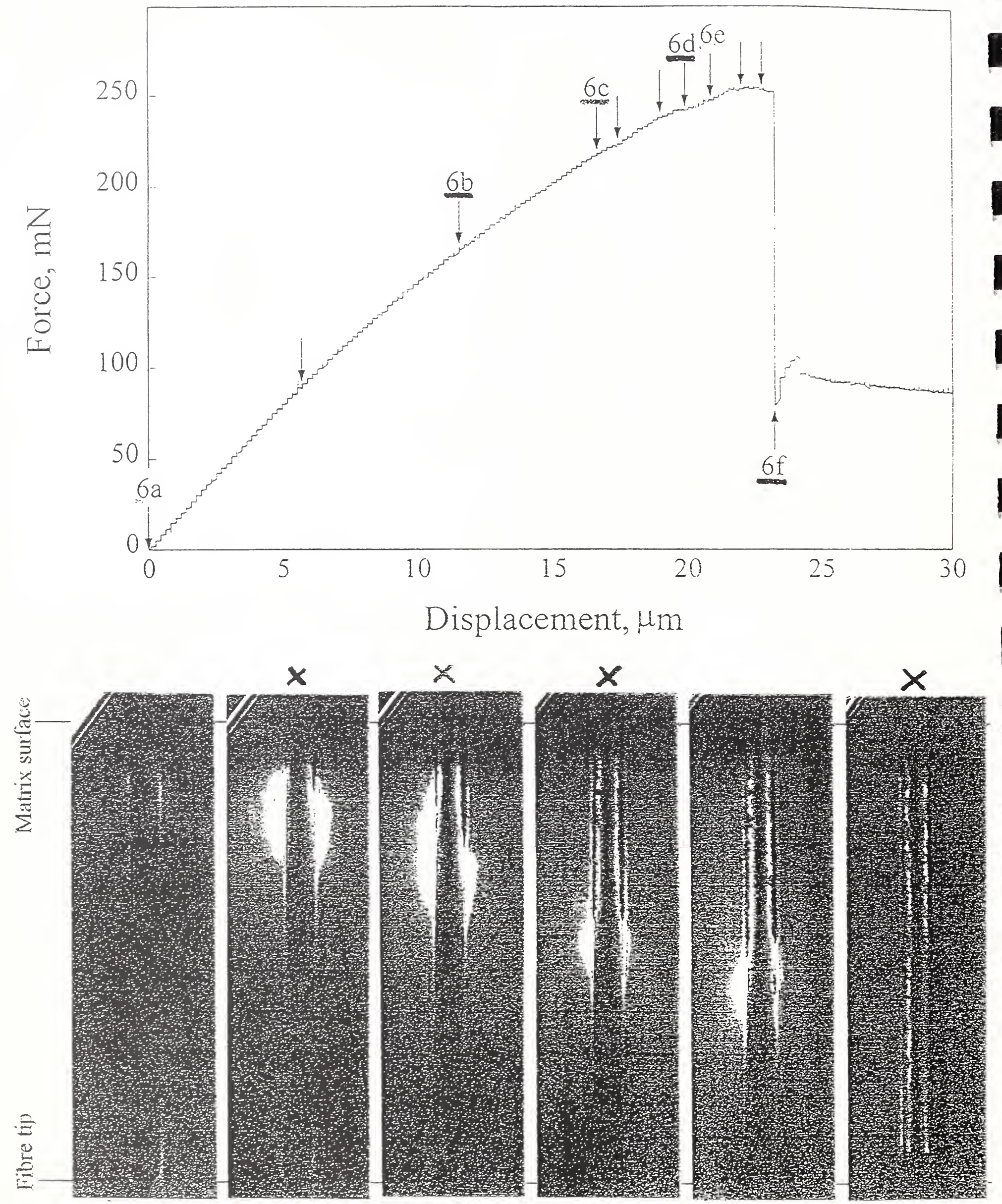


\section{un u! чұбนәา หวедว}

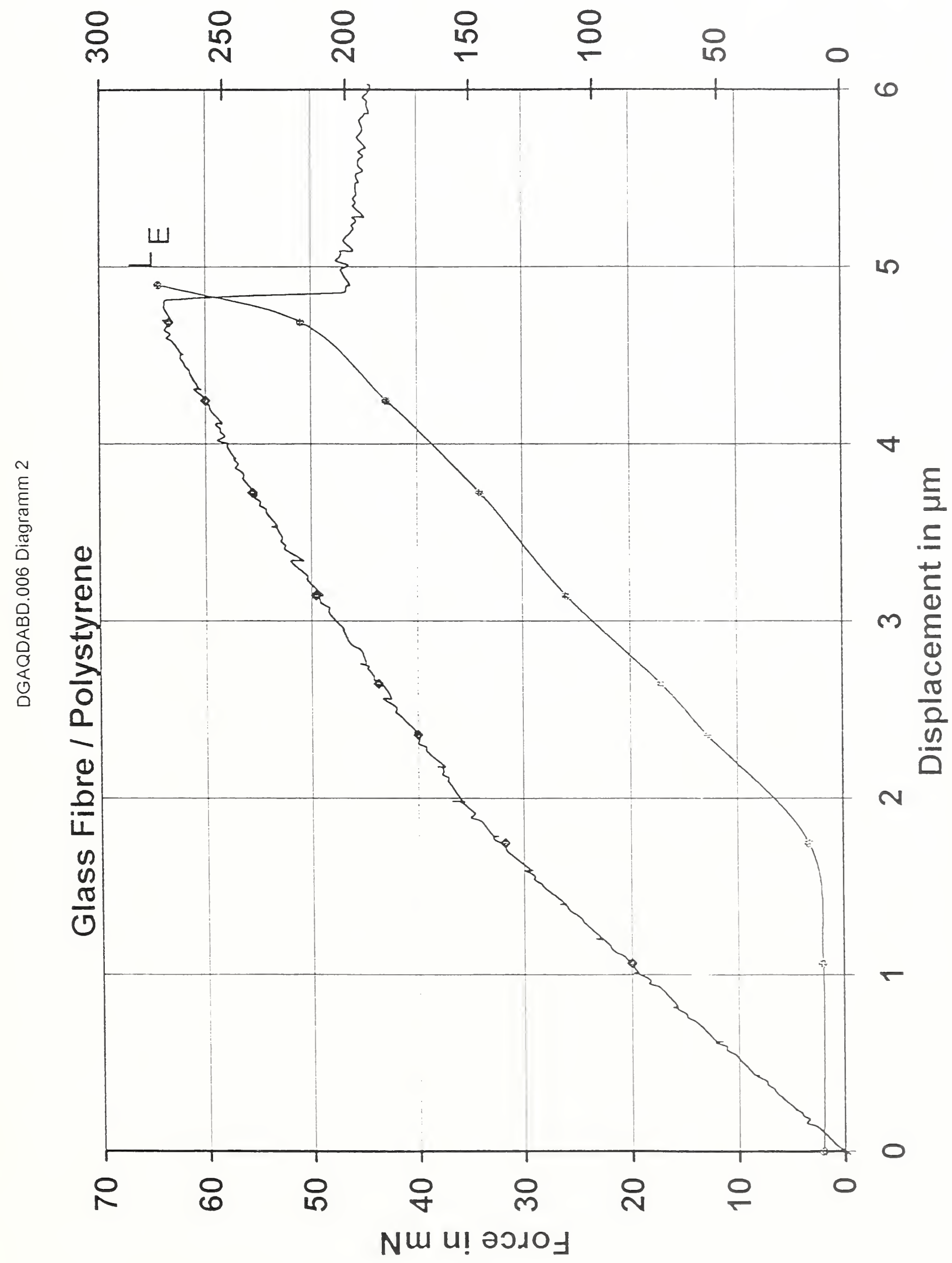




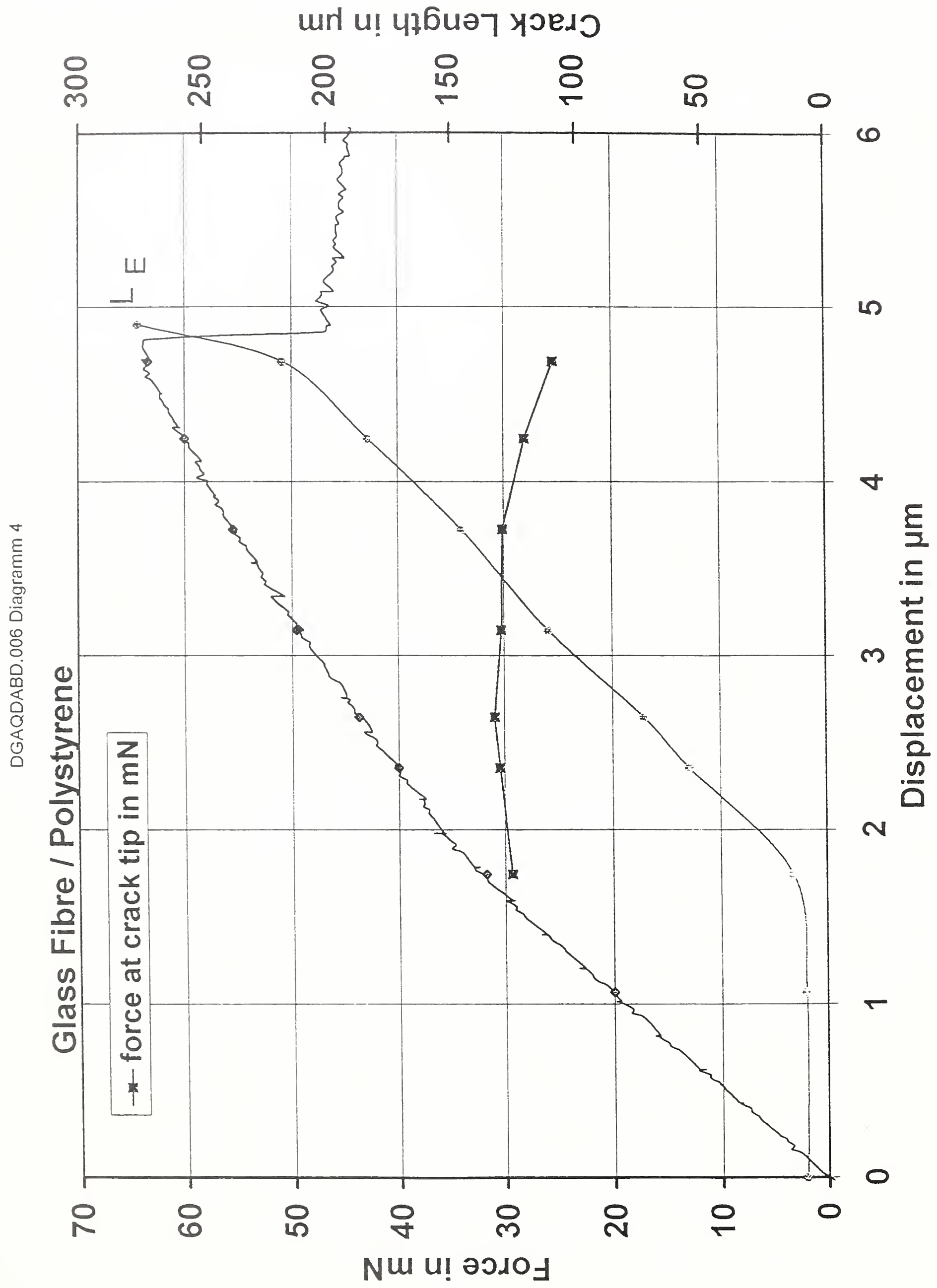




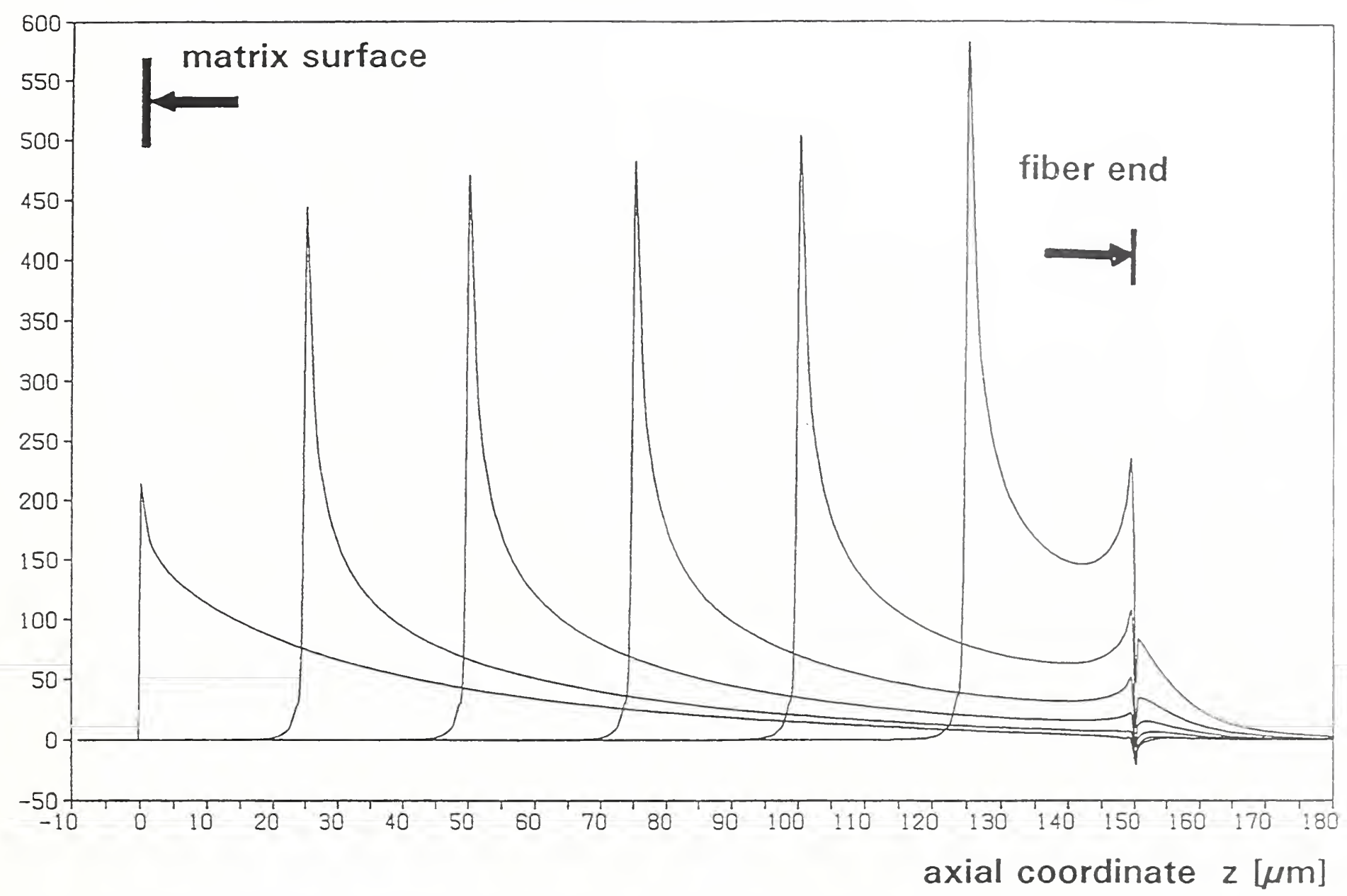

$\sigma_{\mathrm{yy}}\left[\mathrm{N} / \mathrm{mm}^{2}\right]$

Interfacial Radial Stress $\sigma_{y y}$ for different Crack Lengths Load : Pull-out Force and Temperature Load $\left(100^{\circ} \mathrm{C}\right)$

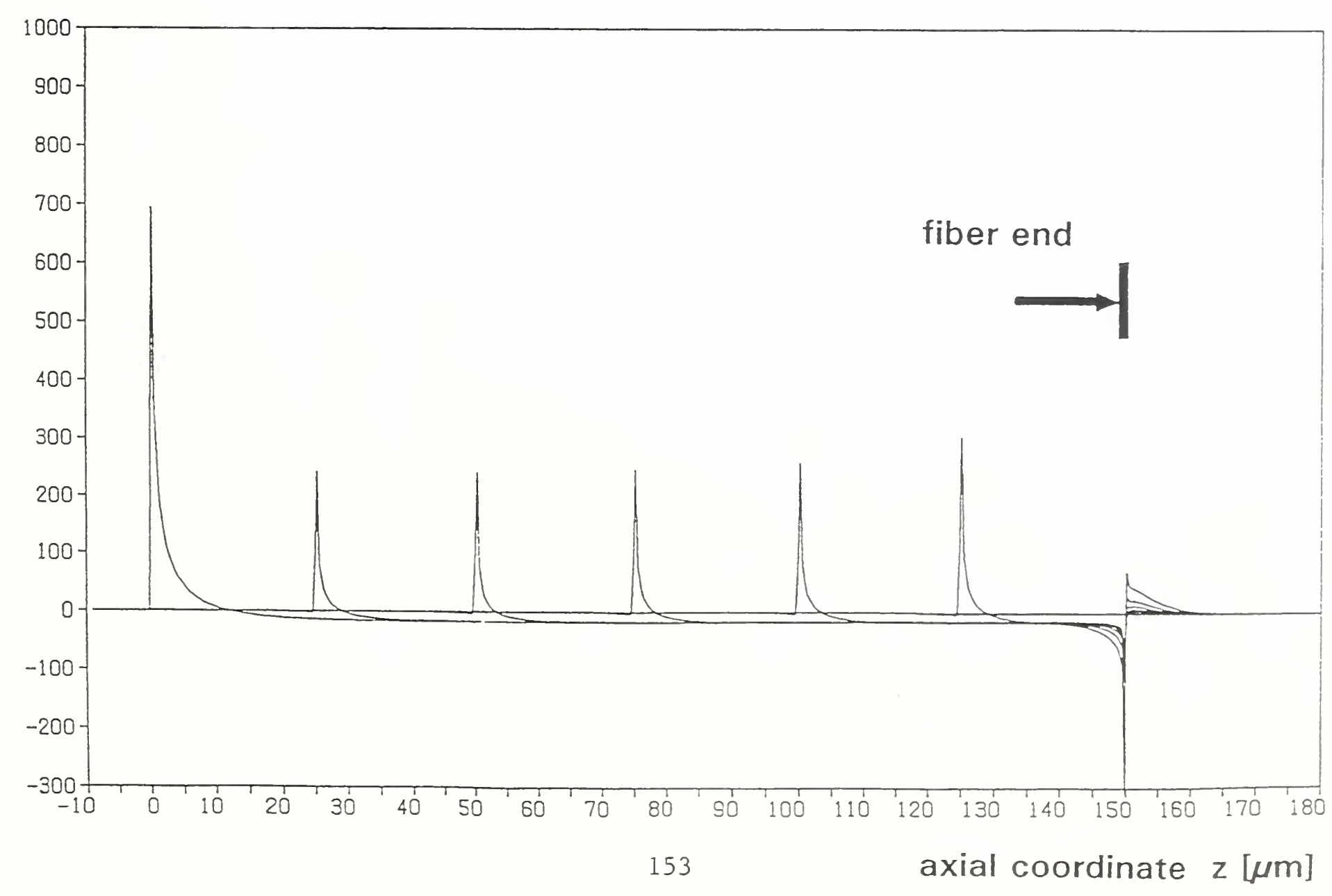




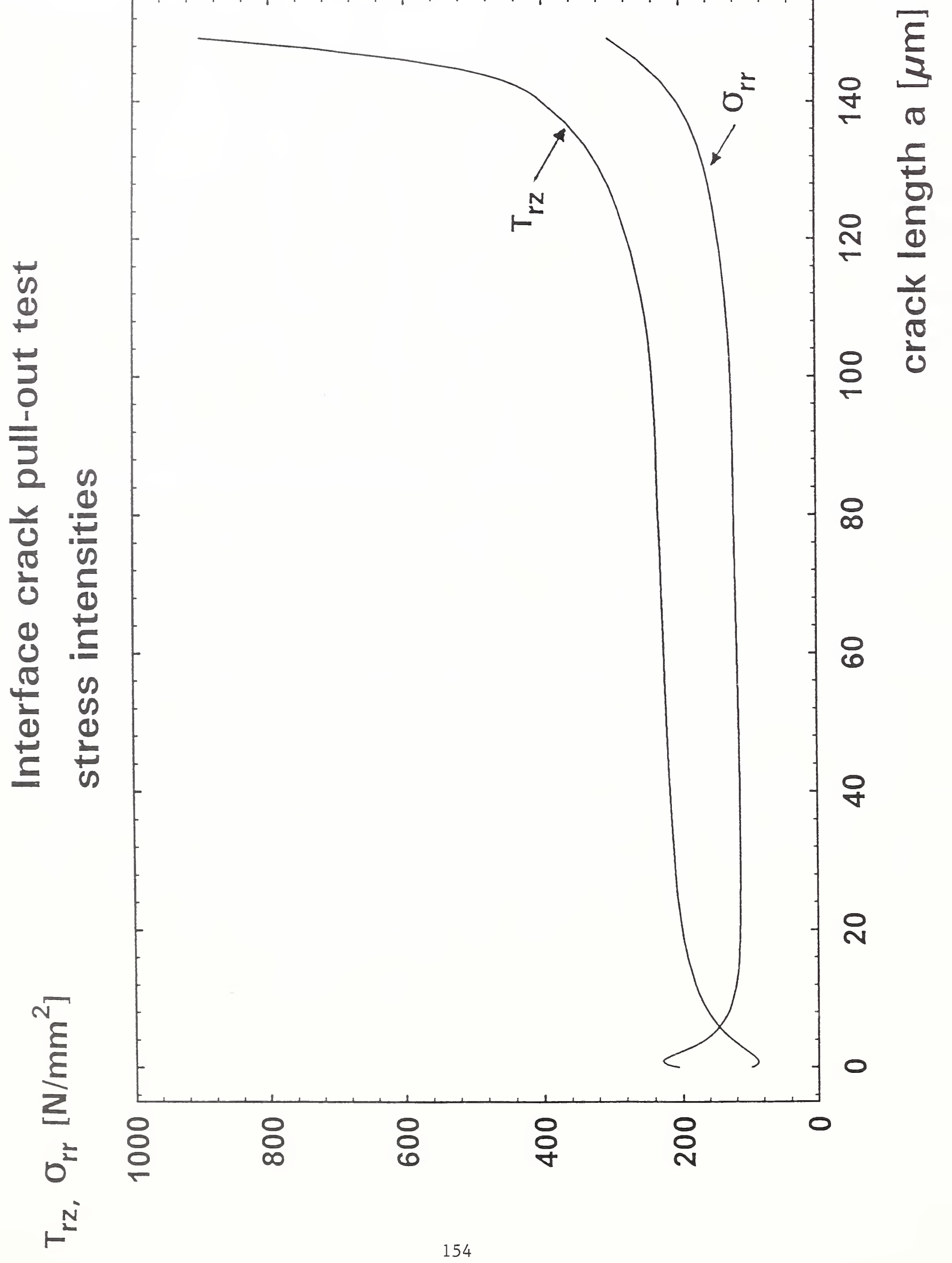




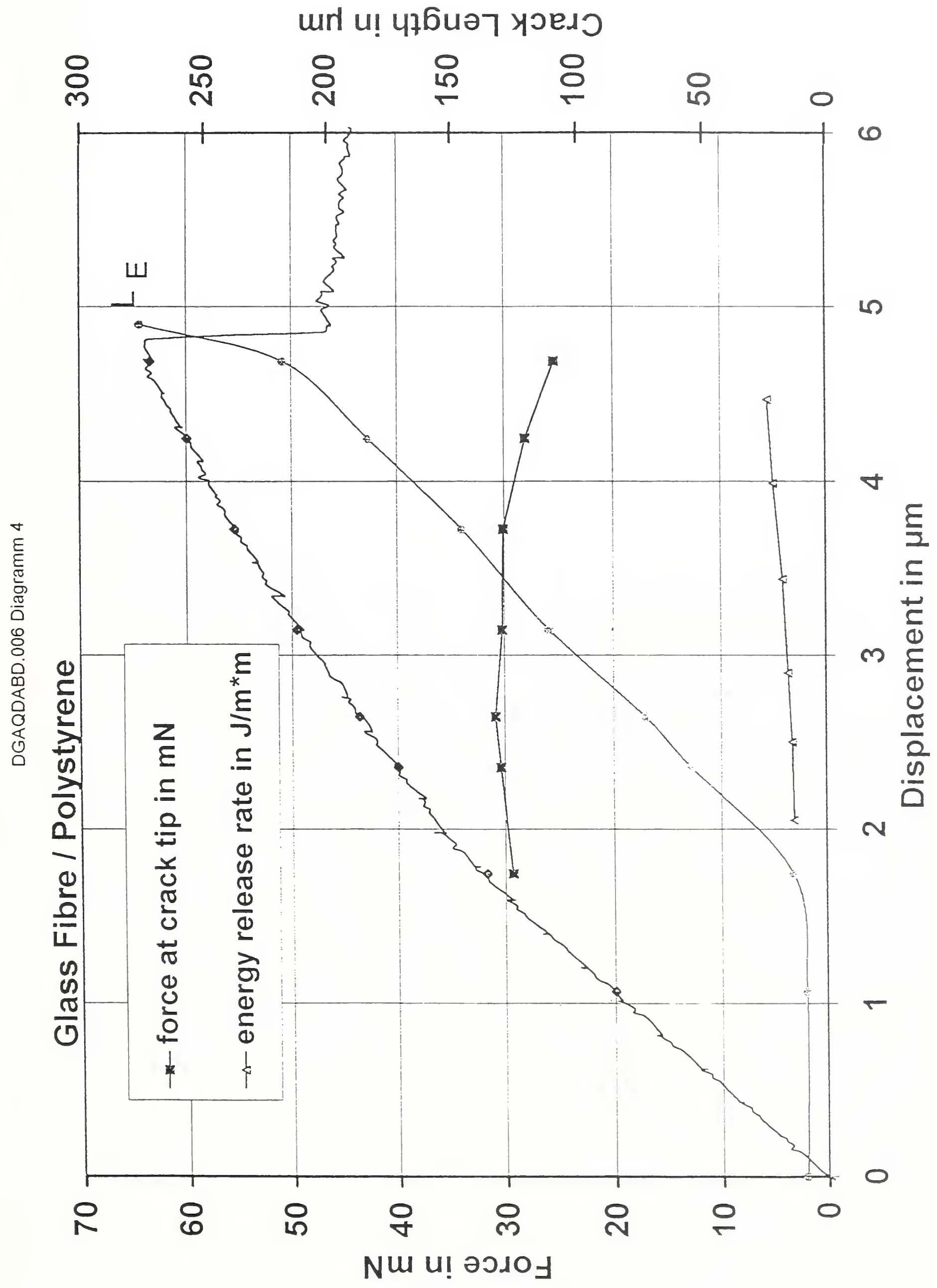




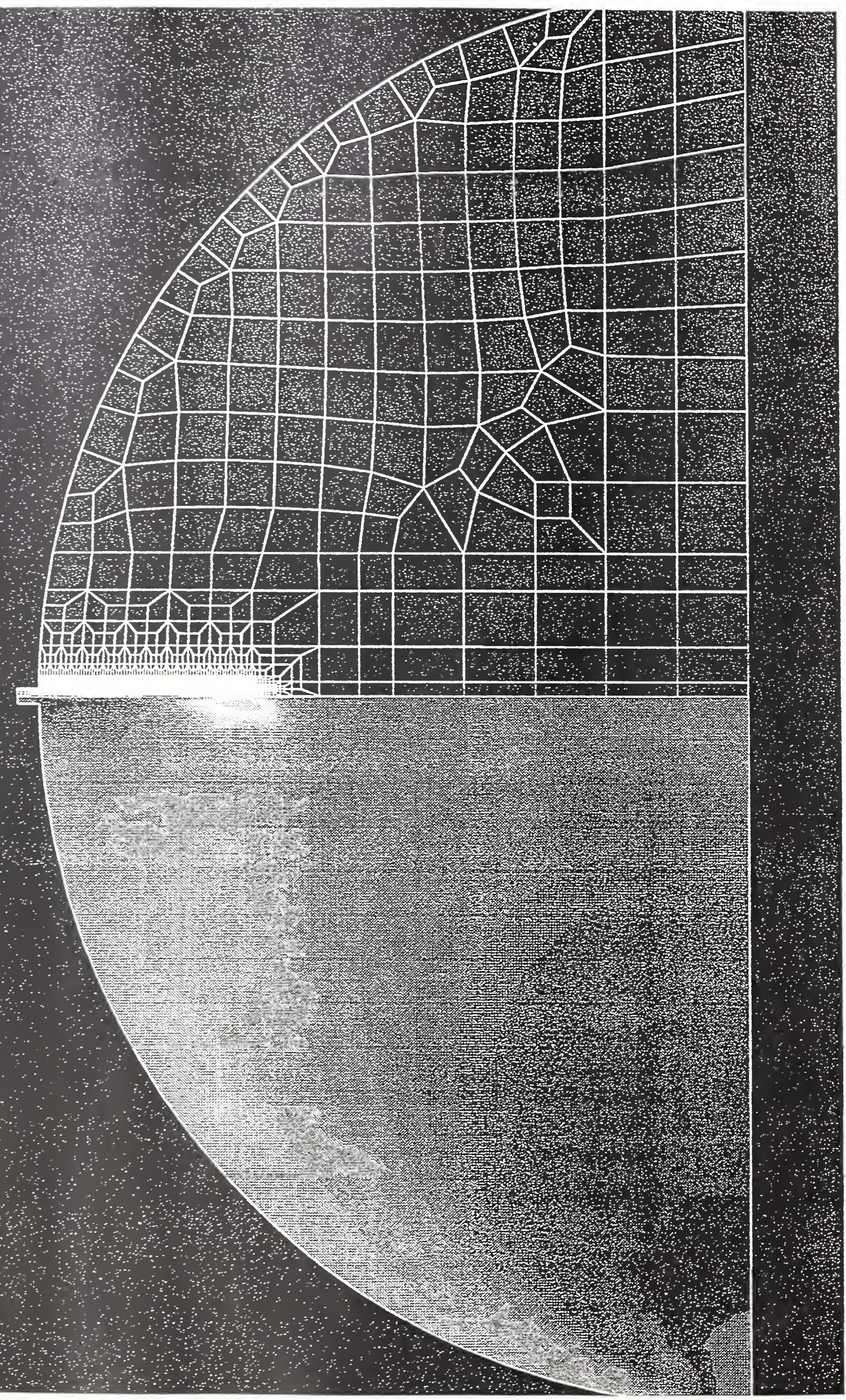




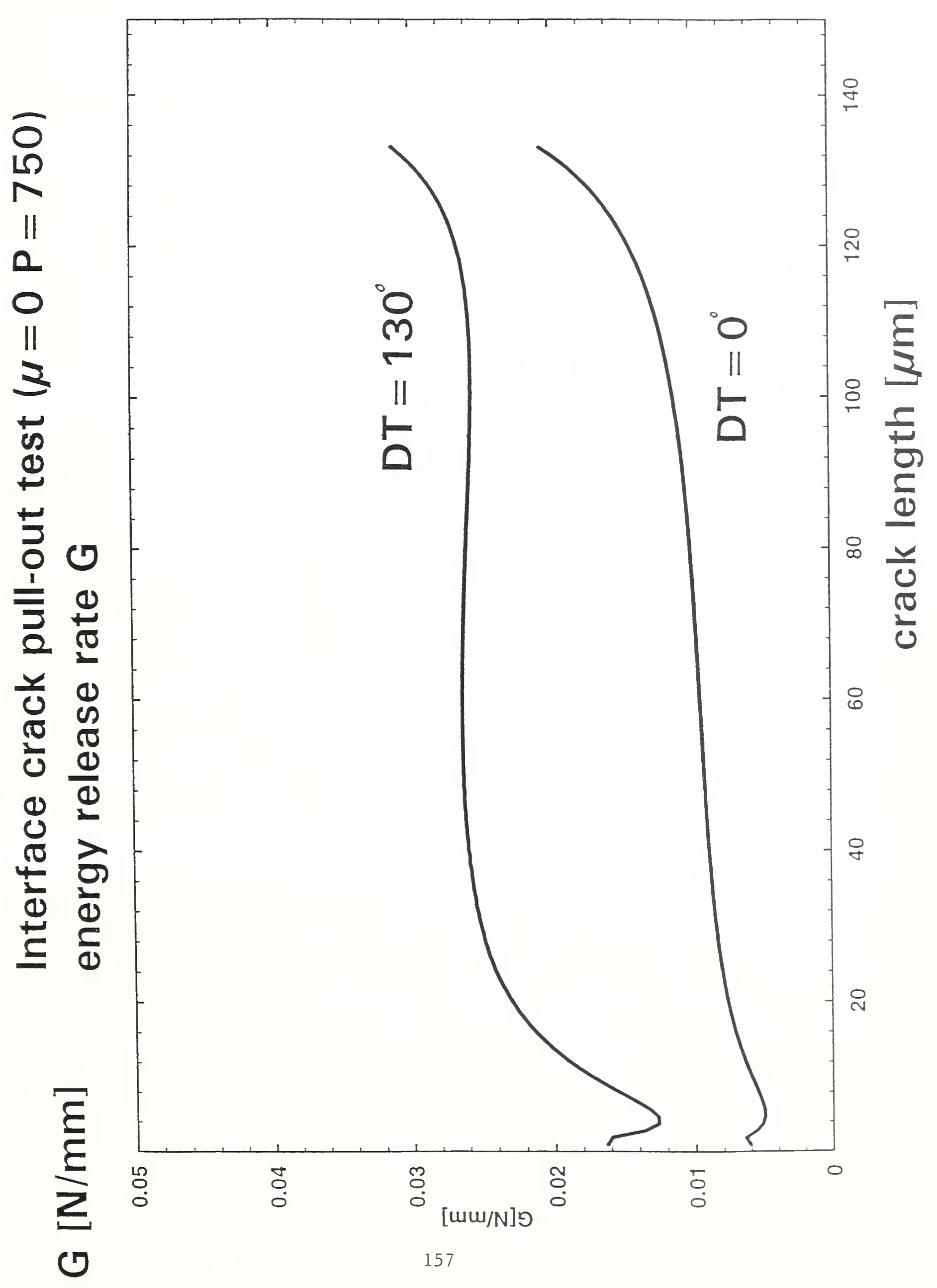




\section{Brittle Failure of the Interface}

Stress Distribution

Indentation Test

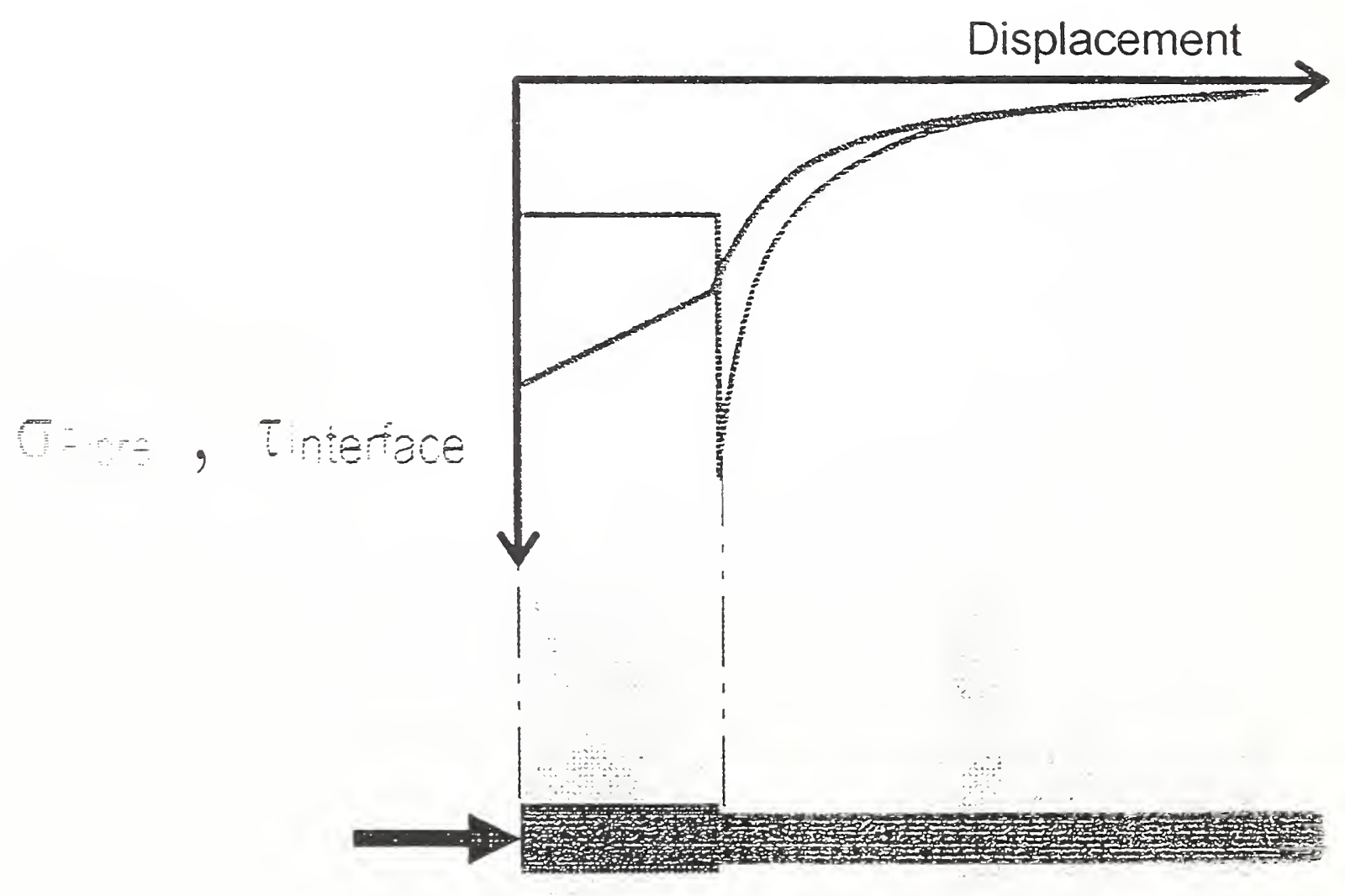




\section{Indentation Test Stress based procedure and interpretation}

\section{Experimental Principle}

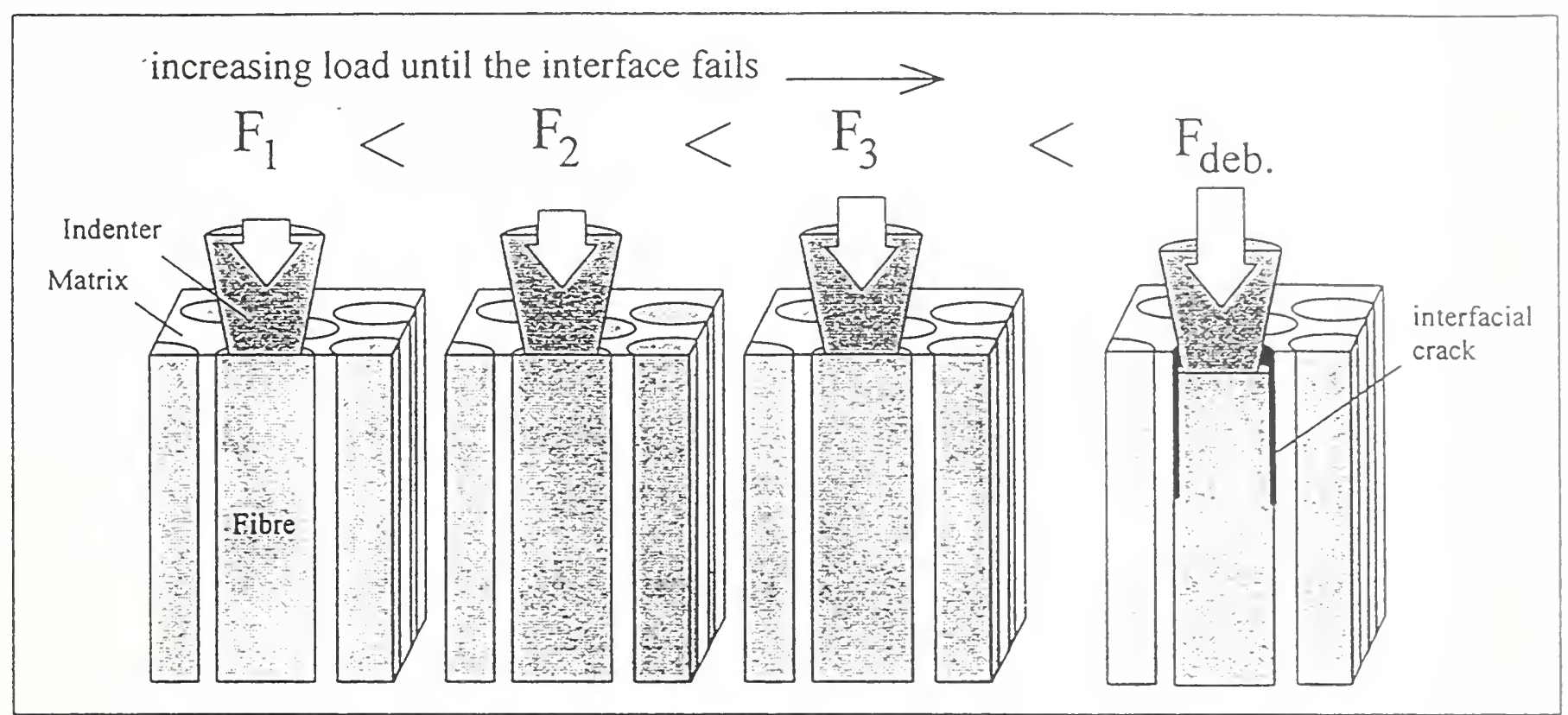

Measurement of the force at first debonding $F_{\text {deb }}$

Calculation of an interfacial shear strength $\tau_{l, u}$ :

$$
\tau_{i, u}=F_{\text {deb }} \frac{\alpha}{2 \pi r_{f} \tanh (\alpha L)}
$$

$\tau_{i, \mathrm{u}}: \quad$ interfacial shear strength

$\mathrm{F}_{\text {deb }}$ : load at the crack initiation

$\mathrm{r}_{\mathrm{f}}$ : fibre radius

L: fibre length

$\alpha$ : shear-lag parameter

P. Lawrence, J. Marter. Sci. 7 (1970) 1-6 


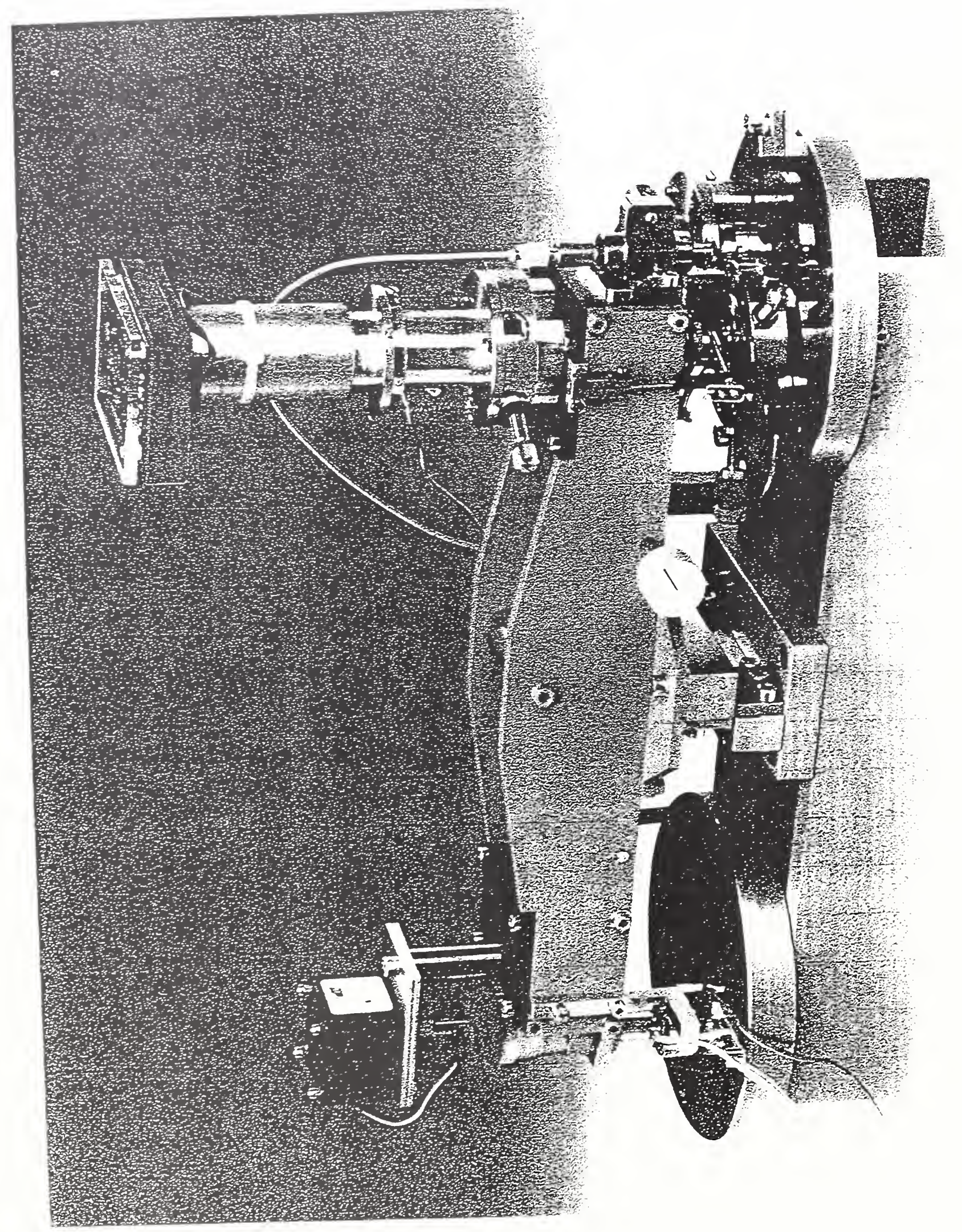




\section{Brittle Failure of the Interface}

\section{Stress Distribution}

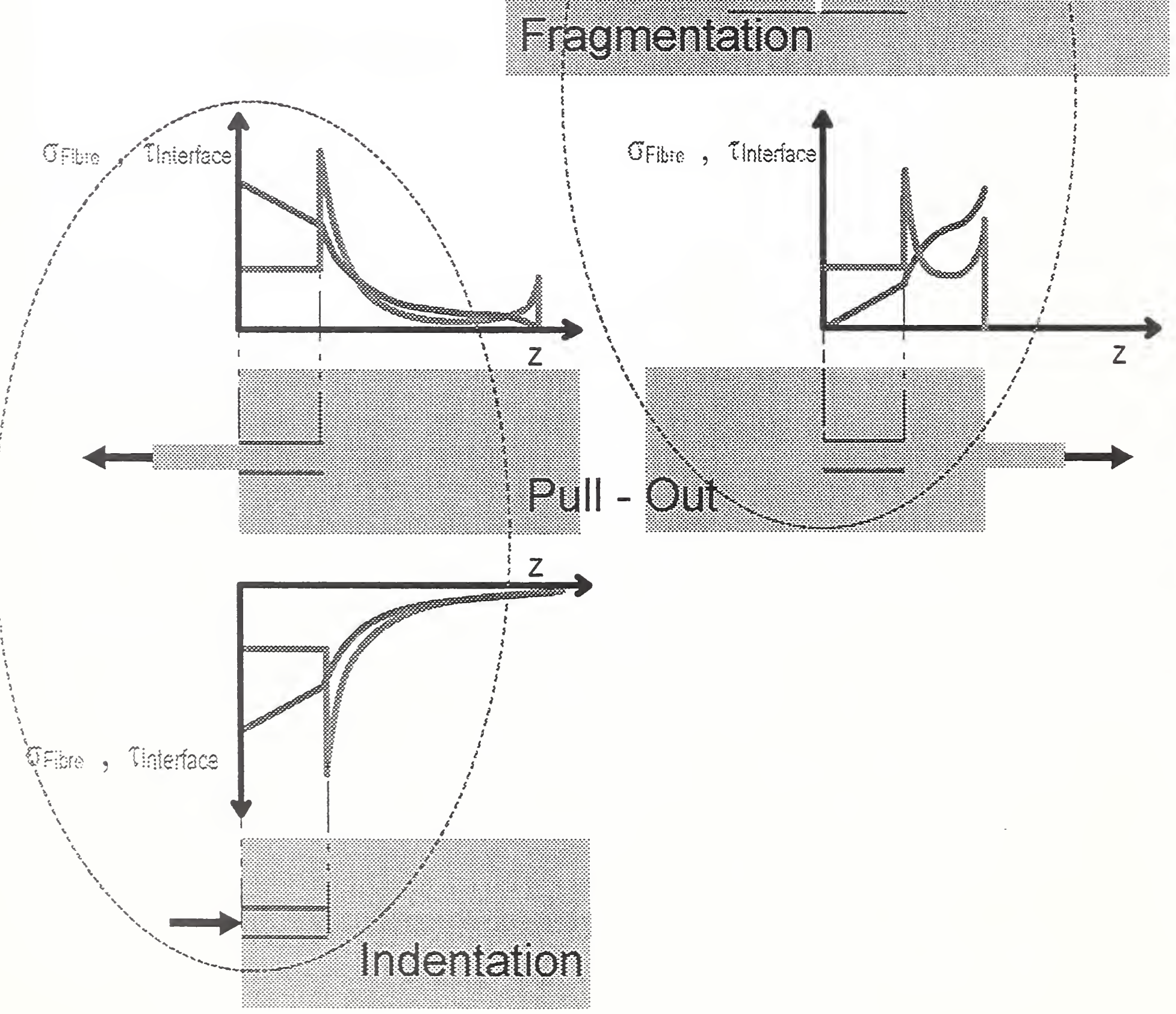




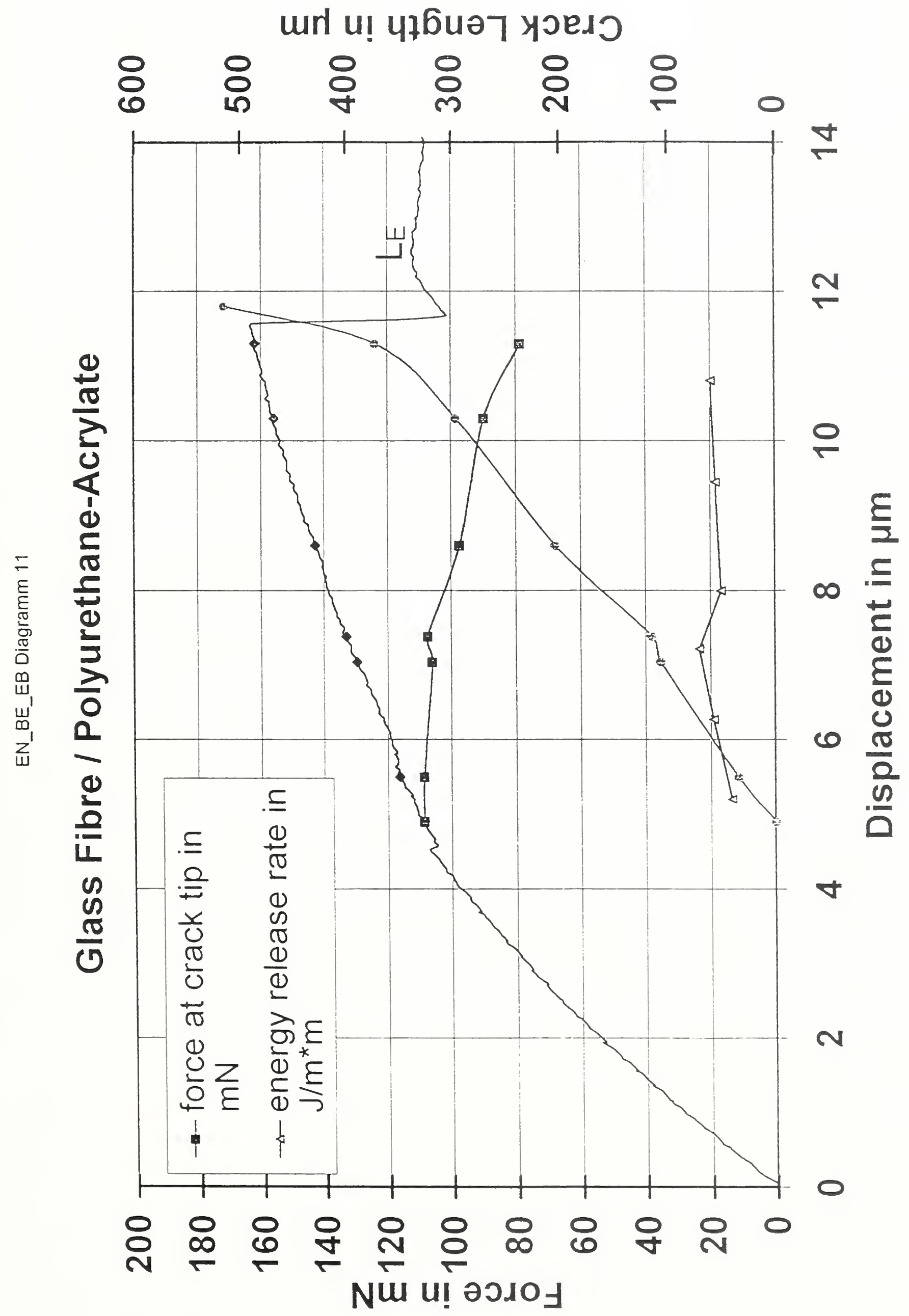




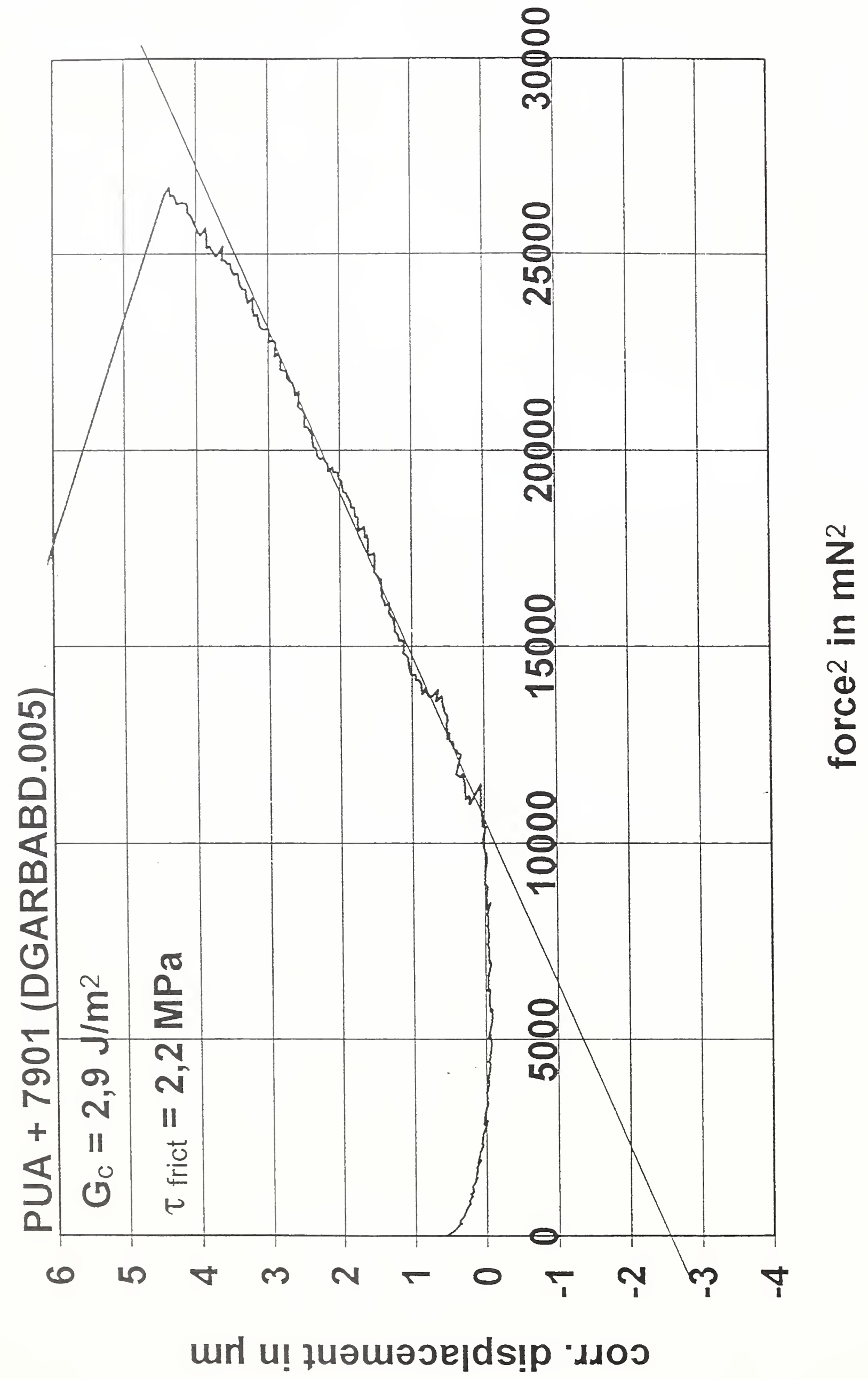


Brittle Failure of the interface

Observables

Fragmentation

\author{
$L$ Crack \\ $\varepsilon$ Matrix
}

Pull - Out

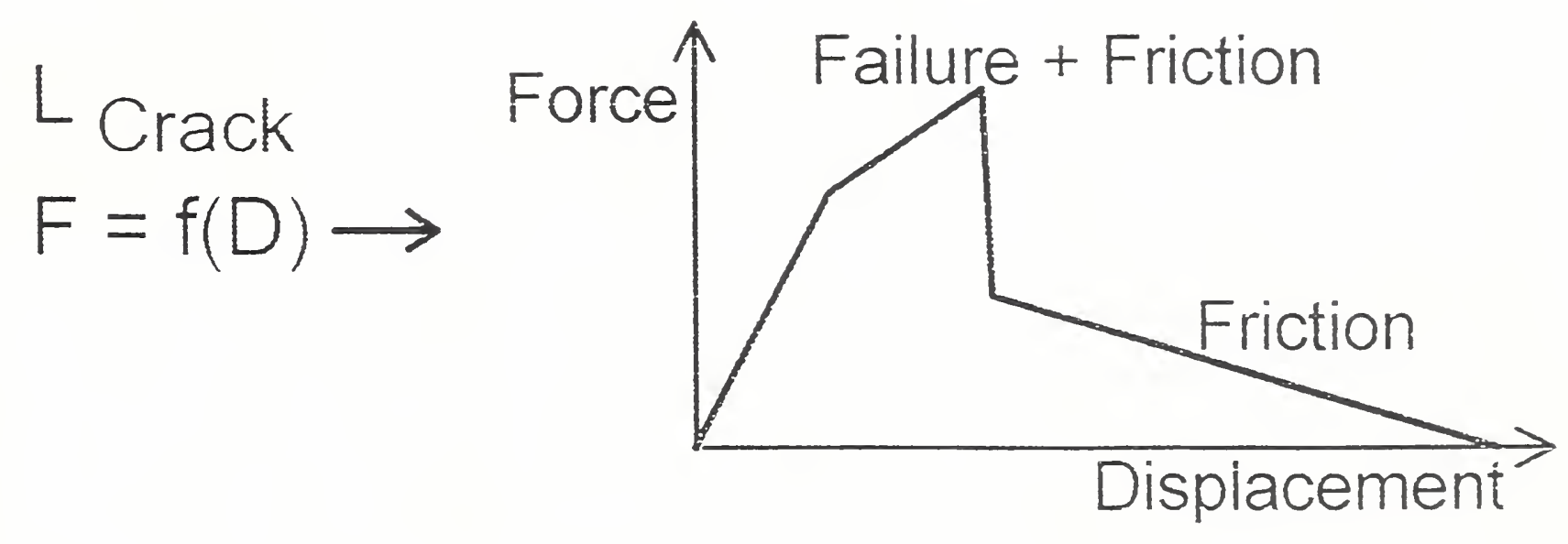

Indentation

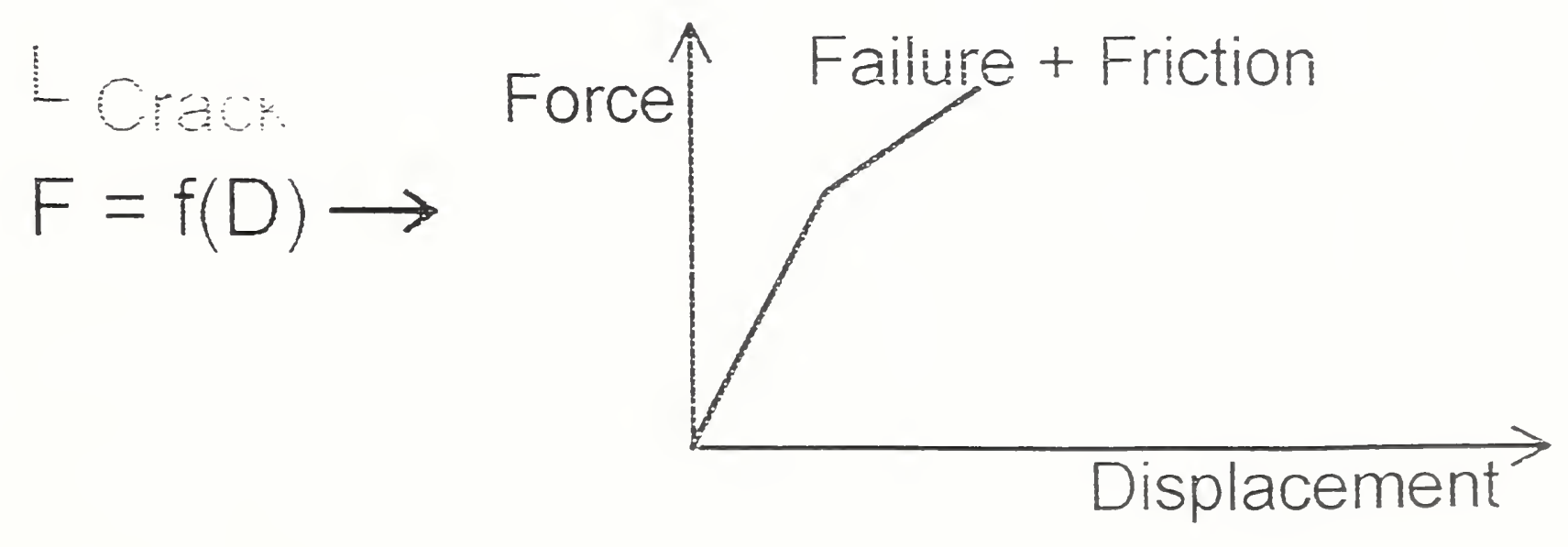




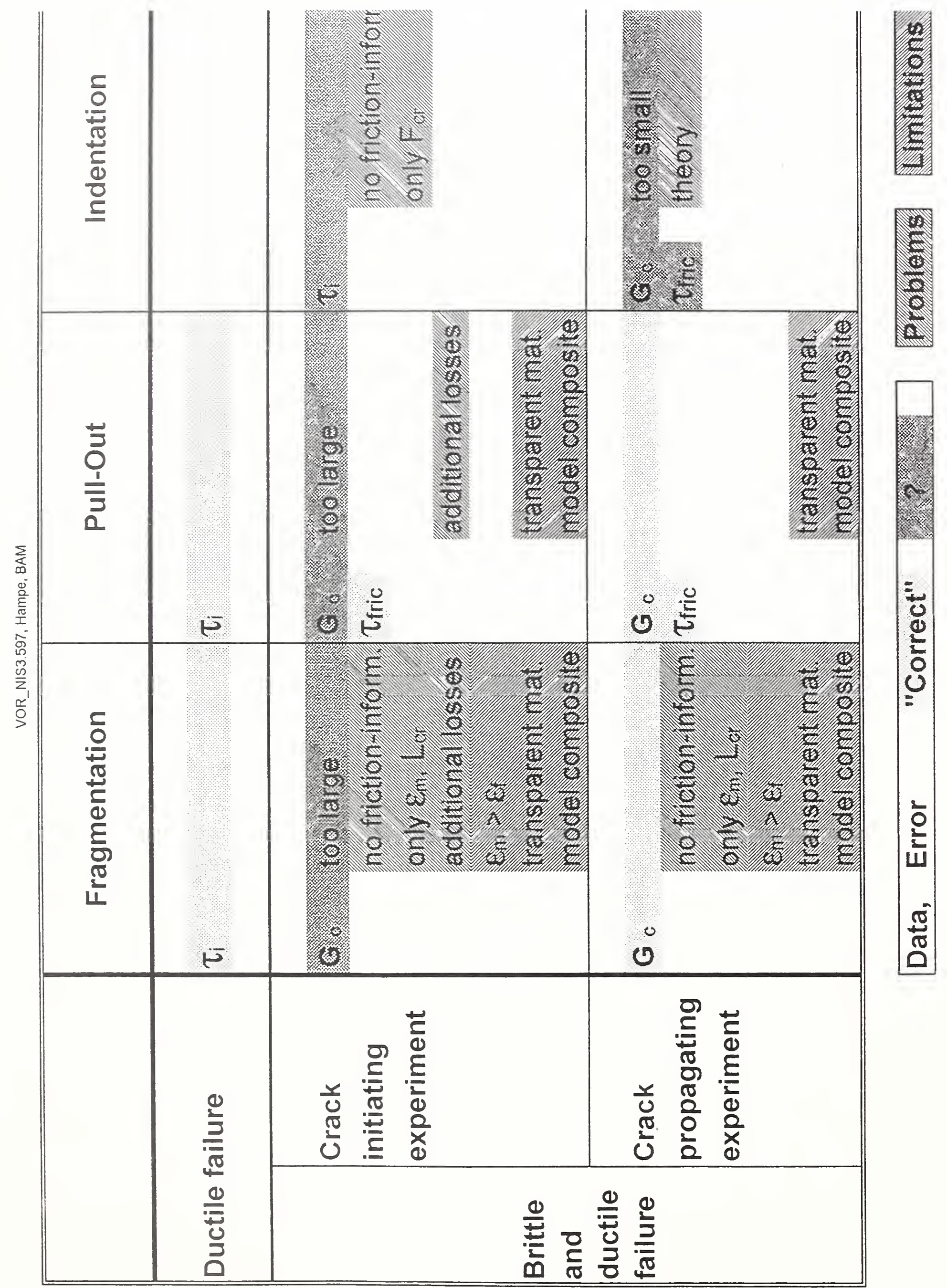




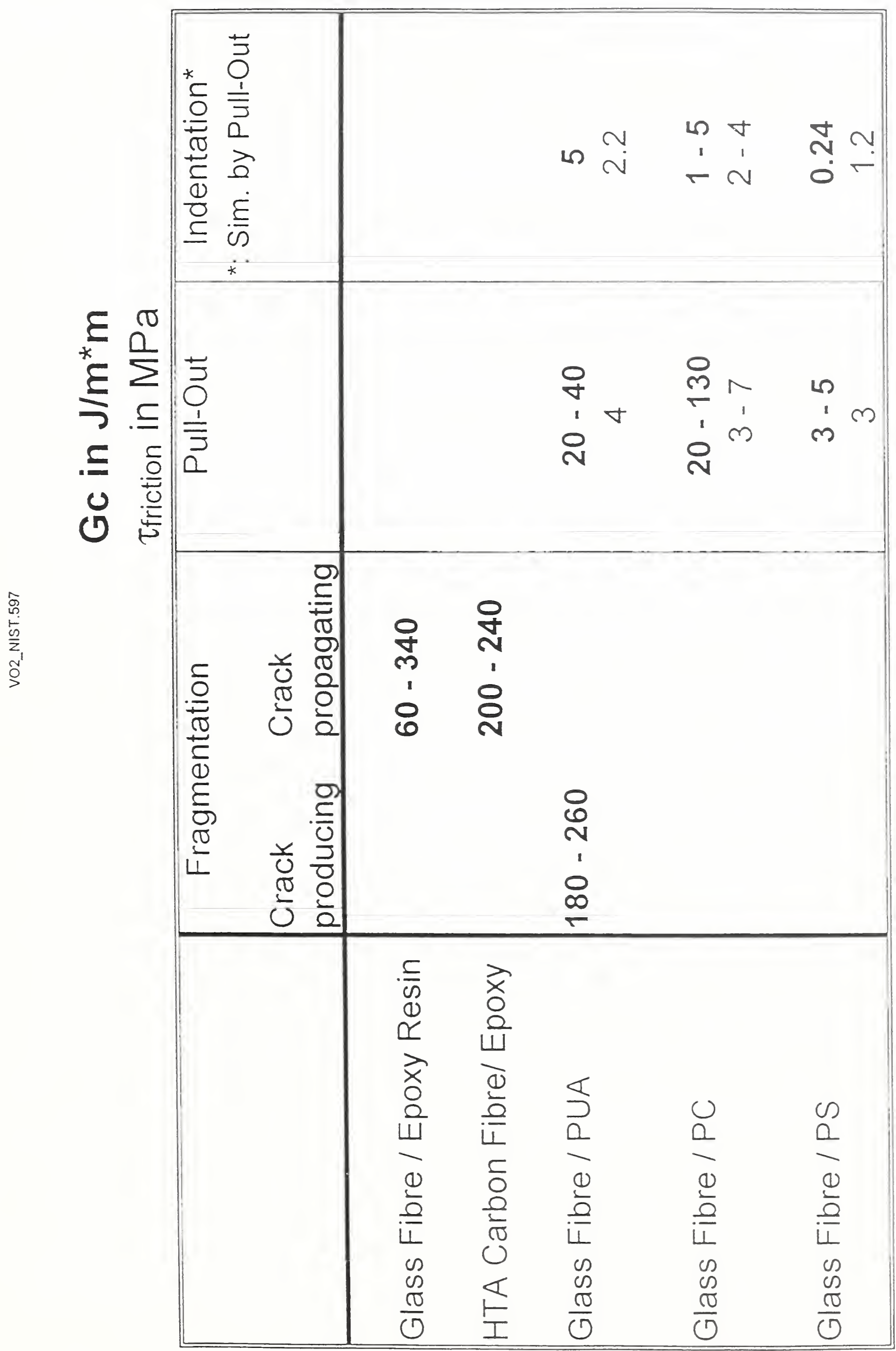





\title{
PERFORMANCE PROPERTY RELATIONSHIPS
}

\author{
Donald Hunston \\ National Institute of Standards \& Technology \\ Polymers Division \\ Gaithersburg, MD 20899
}

\section{INTRODUCTION}

The use of micro-mechanical tests to examine the behavior of the fiber-matrix interface is wide spread. The ultimate objective in such studies, however, is seldom the results themselves but rather the insights that such experiments can provide for the contribution of the interface to the performance of a full composite (Slide 2). ${ }^{1}$ Consequently, one of the most critical questions in this field is the relationship between micro-mechanical test data and the properties of a composite that contains this interface. The objective here is to briefly summarize some of the research directed at this questions and to identify what questions remain unanswered.

\section{Types of Comparisons}

There are three possible levels at which micro-macro comparisons can be discussed (Slide 3). First, there is the level of basic science. It can be argued that the micro-mechanical tests provide basic information about how the interface behaves. Because the these tests generally involve simple geometries, the behavior of this idealized interface is modified by a number of factors and complications that are present in a full composite. For example, the composite can have important fiber-fiber interactions, strong residual stresses, complex local stress effects, and different processing parameters. To relate the micro-mechanical results to full composite behavior at a basic science level, these additional factors must be understood and modeled. Research into these factors is underway--for example, numerous researchers ${ }^{2,3}$ are investigating fiber-fiber interactions--but additional work in this area is clearly needed. Nevertheless, most people would agree that the information provided by micro-mechanical tests will eventually provide some information that will be helpful in understanding composites behavior. Where researchers may disagree is how long it will take to gain sufficient knowledge so micro-macro relationships can be established and how broadly these relationships can be applied.

Since it will be some time before the basic science approach provides definitive answers, two other levels of correlation are often discussed. Both involve direct comparisons of data from micro and macro tests. The first approach looks at relative values and trends. For example, if the interface is systematically altered by a fiber surface treatment, do both the micro and macro tests order the samples in the same way? If so, are the relative changes similar; i.e. if the micro tests indicate that a particular surface treatment improves the interface efficiency by $50 \%$, how is this change reflected in an appropriate composites property? The second approach to comparison looks at the absolute values of the measurements. For example, do various micro tests give the 
same answer, and can one predict composite properties based on the data from micro tests? It is these two approaches that will be considered in this paper.

\section{Uncertainties}

Before beginning such a discussion, however, some comments need to be made about the important the uncertainties ${ }^{4-9}$ that exist for the micro tests and make comparisons difficult (Slide 4). First, there is at the present time no standardized procedure for conducting the micro-tests. This is important because it is known that variations in the testing procedure can produce differences in the results. Second, in order to compare macro-test results with data from macrotests, it is useful to know exactly what property is measured in the micro test. A number of analysis methods have been developed based on different assumptions about the test, and most will give different results. One of the important differences is whether the failure process is assumed to be a fracture event or a strength/yielding event. A third complication is that although the tests are often called shear measurements, the stresses are not pure shear. It is well know that the failure behavior depends on the load mix so this can be important. Moreover, since the different micro tests have different local stress fields, it can be argued that each method measures a somewhat different property. In additions to uncertainties about the micro tests, there are questions about how the information translates into composites behavior. For example, what macro tests should be used for comparison? Moreover, since the macro tests often measure complex behavior, how should the data be analyzed to get the most appropriate comparison with micro results? All of these questions are important topics for current and future research.

\section{TYPICAL RESULTS}

Despite these uncertainties, many studies ${ }^{10-24}$ have been conducted in this area, and they provide useful insights. The discussion here will try to summarize some of what has been learned. Data from the work at NIST will be used to illustrate the various points, but these results are typical of studies in many other laboratories. This examination of micro tests will be made at three levels: the micro tests themselves, micro-macro test comparisons, and use of the tests in durability studies. The durability discussion is divided into two parts: coupling agent studies and micro-macro comparisons (Slide 5).

\section{Micro-micro studies}

There have been many studies that examine the relevance of micro tests by focusing on the tests themselves. Some compare results from different micro tests to look for consistence. A wide variety of micro tests have been developed (Slide 6 and paper by Drzal), and many of these have been used in such studies. Other investigators have compared micro test data to expectations for cases where the changes should show particular trends. For example, if the fiber surface is treated to improve bonding, the micro tests would be expected to show improvements in interface strength. Finally, correlations have been made with other characterization tests like work of adhesion. 
One example of micro-macro comparisons is the work of Herrera-Franco, et al ${ }^{15,16}$ who used the fragmentation, bead pull-off, and indentation (Slide 7) experiments. Their studies used samples made with epoxy (Epon 828, Shell Chemical Co.) and two types of carbon fibers (AS-4 and IM-6, Hercules, Inc.). For each of the two fiber types the surface treatment was also varied. In the first case, the AS-4 fibers are given a commercial electrochemical oxidation step designed to increase adhesion with epoxy, while the AU-4 fibers were prepared in the same way but without this final oxidation step. In the second case, IM6 type fiber that were not given a special surface treatment after fabrication were designated IM6-U. The fibers labeled IM6-100 and IM6600 are identical to IM6-U but were given a commercial surface treatment to improve bonding; the surface treatment for IM6-600 was 6 times as long as that for IM6-100.

Slide 8 shows the results for AS-4 type fibers obtained by the authors after analyzing the micro-test data with a simple Kelly-Tyson ${ }^{25}$ approach. All of the tests indicated that the AS-4 samples had a stronger interface just as one would expect. Moreover, the magnitudes of the improvement shown by the tests were similar, about two fold. The indentation tests indicated a smaller improvement here, but in other experiments of this type, the indentation test has shown a sensitivity to different samples that is similar to the other two tests. The final observation worth making from these results is that the absolute values of the strengths measured in the three tests are similar but not the same. In light of the very simple Kelly-Tyson analysis that was used and the fact that the stress fields for the three tests are different, this is not surprising.

Slide 9 shows the results for the work on IM6 type fibers. Here again, samples made with fibers having surface treatments which should produce better bonding gave higher numbers in the micro tests. The one exception was the IM6-600 sample for the micro-drop test. The authors explained this by noting that the curing agent used to prepare the samples has some volatility. Because the experiment employs tiny which have a small volume and high surface to volume ratio, a significant fraction of the curing agent was often lost through volatility during processing. One indication of this is that the drops were frequently under cured and had $\mathrm{T}_{\mathrm{g}}$ 's that were different than that for larger samples. This illustrates what is perhaps the biggest challenge for some of the micro-tests with particular resin systems. Because the processing with some resins and test samples can be different than that used for a full composites, the micro-test samples and composites may have significant differences in features like cure state, fiber wetting, and microstructure (Slide 10). Such variations can affect interface behavior so the relevance of micro-test data for composite behavior is questionable. Consequently, where processing factors are important, the micro test samples need to be fabricated in a way that simulates the composite processing. In some cases this may not be possible so the only useful sample is a composite. This represents an important advantage for the micro-indentation test which uses composite materials On the other hand each of the micro tests has different strengths and weaknesses so as a general rule, it is desirable to have multiple micro tests available.

${ }^{2}$ Certain commercial materials and equipment are identified in this paper in order to specify adequately the experimental procedure. In no case does such identification imply recommendation or endorsement by the National Institute of Standards and Technology, nor does it imply necessarily that the items are the best available for the purpose. 


\section{Micro-macro comparisons}

Another way to examine the meaning of micro-test results is to make a comparison with data from full composites. Despite the obvious importance of such comparisons, the number of studies in this area is quite limited. One reason is that such comparisons are complex. The most comprehensive approach is to predict composite behavior based on values obtained from microtests. Unfortunately, in addition to the uncertainties discussed above for the micro-tests, there are also questions about how to translate the micro-test results into macro-test predictions. As mentioned by Drzal and several others in this meeting, few mechanics models even include an interface in their description of composite behavior. Consequently, there are important questions about what composite tests to select and how to make the prediction. A comparison of this type will be discussed in the durability section, but there is also a more empirical approach that has been discussed in the literature. In this case, it is usually assumed that the micro tests provide a measure of interface shear behavior so the most appropriate composite experiments are those that measure interlaminar shear. One example of such a comparison was performed by Madukar and Drzal. ${ }^{17}$ They made their samples with the same fibers, matrix system, and processing conditions as the work cited above. Three composite tests were used: $\pm 45^{\circ}$ tension, Iosipescu shear, and short-beam shear (Slide 11). For the comparison to be meaningful, it is important that the failure in these tests actually involve shear, and the authors restricted themselves to those cases. Slide 12 shows their results along with data from the micro tests. The conclusions are similar to what was found above. Trends are consistent in that the AS-4 samples always gave higher results than the AU-4 samples. In addition, the relative improvements were similar, about 1.8 fold. As might be expected, however, the absolute numbers do not agree.

\section{Durability-coupling agents}

Another area where the usefulness of the micro tests has been examined in durability. ${ }^{18-23}$ In glass reinforced composites, the fiber-matrix interface is known to be susceptible to attack by moisture, and thus interface studies are relevant. NIST has been studying durability for a number of years, and their results are illustrative of research in this area. Two studies will be summarized here: the first focus on the use of coupling agents to improve durability while the other compares results from micro and macro tests on samples made using the same procedure. Both studies use E-glass fiber (Owens Corning) and epoxy (Epon 828, Shell Co.). The samples were aged in either air or water at $25^{\circ} \mathrm{C}$ or $75^{\circ} \mathrm{C}$, and the interface strength was monitored with the fragmentation tests (Slide 13). One complication with the fragmentation test is that the fiber strength is needed to calculate interface strength, but moisture degrades the fiber as well as the interface. The only solution is to use the fragmentation test to monitor both properties. A number of authors ${ }^{18}$ have addressed this challenge, and the results show that the low stress data can be used to estimate fiber strength, and this can be combined with the high stress data to estimate the interface strength (Slide 14).

The first study to be discussed here focused on the effect of coupling agent. ${ }^{19,20}$ Two coupling agents were used: 3-aminopropyl triethoxysilane (APS) and n-octadecyl trichlorosilane (OTS). Both react with glass but APS reacts with epoxy as well while OTS does not (Slide 15). By using these materials individually and in mixtures, the molecular bonding between the coupling 
agent and the epoxy can be systematically varied. Concentrations in Slides 16 and 17 are given in mole fractions of APS and/or OTS. This also represents the fraction of non-Si end groups which can react (APS) or not react (OTS) with the epoxy. The classic work of Argon and Gent ${ }^{26}$ shows that on flat plates, the strength of the interface varies in direct proportion to the amount of molecular bonding. Slide $\mathbf{1 6}$ shows some of the results for the initial (dry state) fiber-matrix interface strength. Just as in the Argon-Gent study and other work with flat plates, increasing the molecular bonding (more APS) increased the interface strength while a clean glass surface with no sizing gave intermediate interface strength. When the fragmentation samples are aged in water, the data analysis indicated that the fiber strength did not degrade but the interface strength did (Slide 17). With samples made using unsized surface (no coupling agent) rapid degradation was observed, while the samples made using fibers coated with APS showed no loss of strength. Because there are no corresponding data for full composites, it is impossible to say if these data are representative of what would happen in a composite. Nevertheless, it is worth noting that these conclusions are exactly what we would expect for the behavior of coupling agents on flat plates. The results are therefore encouraging with regard to the relevance of fragmentation tests to composite behavior.

\section{Durability-micro-macro comparisons}

The second durability study ${ }^{21}$ used the same resin and E-Glass fiber but with two commercial sizings: one designed for epoxies the other for vinyl esters. The fragmentation studies were done in the same way while four tests were performed on the full composites (Slide 18). The $0^{\circ}$ tension tests was selected to monitor degradation of the fiber while compression and Mode I and II interlaminar fracture were designed to test the interface. Slide 19 shows the fiber strength data from the fragmentation test and tensile strength measurement. The trends are strikingly similar. Both tests show identical strength degradation patterns: rapid initial drop followed by a slow decrease in strength, little effect of coupling agent choice, but a strong influence of temperature. When an attempt was made to predict the composite behavior from the micro-test results, however, the success is disappointing (Slide 20). Although the trends are clearly there, the predictions overestimated the strength reduction. The authors cite three factors contributing to this failure. First, the models used in this work to predict composite strength were quite simple. More detailed models are available in the literature, and use of such models might improve the predictions. Second, more extensive data needs to be taken in the fragmentation test improve the reliability of the analysis. Finally, the analysis of fiber strength from the fragmentation results could be significantly improved with a better model.

Results for the interface strength are more difficult to analyze. Consider first the fragmentation results. The calculated interface strengths showed a large scatter, but the degradation was generally slow. The test condition and sample that displayed the largest loss of properties was emersion in water at $75^{\circ} \mathrm{C}$ for the sample with vinyl ester coating (Slide 21). Although four different composite tests were performed, only one example will be discussed here, mode I interlaminar fracture behavior. Typically, interlaminar tests produce results for fracture energy such as shown in Slide 22. The sample is loaded, and at some critical value of the fracture energy, the crack begins to move (initiation). As the crack grows, the resistance to growth increased so a higher fracture energy is needed to keep the crack advancing. Eventually a steady 
state is reached, and the crack continues to grow at an approximately constant fracture energy. This is called R-curve behavior and is attributed to the fact that the crack starts in a resin rich region, but as it grows, fiber bridging becomes important. Fiber bridging occurs when fibers from the ply on one side of the crack plane cross over to the ply on the other side of the crack plane. As the crack advances, the bridging fibers must be broken or pulled so the resistance to crack growth increases. Slide 23 shows the results for Mode I interlaminar fracture of the composites at various aging times in water. There was relatively little effect for the samples with epoxy compatible sizing, some effect for the vinyl ester sample aged at the low temperature, but a sizable change for the vinyl ester sample aged at the high temperature. These trends are similar to but not identical to what was observed in the fragmentation test.

In all cases the initiation values showed little change. The expectation is that the resin toughness increases with water pickup while the interface strength does down. The two effects would tend to counteract each other, but it is surprising that the initiation value didn't change at all. The steady state toughness could be affected not only be resin toughness and interface strength but also by fiber strength. If the fiber is weaker, the bridging fibers can be more easily broken thus reducing the resistance to crack growth. In this case, however, it would appear that interface strength dominated since the loss of fiber strength was independent of fiber coating while interface strength and plateau fracture energy were not (Slide 23).

\section{CONCLUSIONS}

The examples cited here are typical of what has been seen and published by many researchers. The overall conclusions (Slide 24) at the present time are that the micro tests can provide useful information relative to composites. The results are best suited for ranking material systems, but the relative values can also be useful. On the other hand the absolute results seldom agree. Each micro test method has its own strengths and weaknesses so having a variety of techniques is advantageous. It must also be mentioned however that the correlations do not always work. Perhaps the most critical problem is that it is sometimes difficult or impossible to fabricate the micro-test samples in a way that simulates the processing of full composites (Slide 25). In some cases this may not matter, but in others it can produce differences in morphology, wetting, composition, etc. which will alter the interface behavior. Under these conditions, the micro test results may be of little use in understanding or prediction composite behavior. In addition to the processing effects, there are other problems. As mentioned earlier, it isn't always clear what the micro test measures and what analysis to use. The examples cited here use a simple Kelly-Tyson approach, but many researchers have improved on this method with more sophisticated and realistic analyses. Unfortunately, the different analysis methods give different results so a choice must be made as to which is most appropriate for a given system. In some cases, none of the available analysis methods may be adequate. Once a value is obtained from the micro test analysis, it must be translated into composite behavior. As discussed above, this involves a number of important questions. Consequently, one of the most important needs in this field is to improve the analysis capability for both the micro and macro tests. To reach this goal, better experimental data and an improved understanding of the tests must also be obtained. Part of this process is the need to standardize the micro test methods. 


\section{Standardization needs}

The need for standardization is best illustrated by the results of a round robin ${ }^{24}$ conducted in the early 90's. Twelve different laboratories agreed to measure interface strength for a common fiber-resin system. The three tests discussed above were used as well as the fiber pull-out test. Slide 26 shows just one set of results from this study. There was a very wide variation in the results when different test method were compared, but as discussed above, this may be understandable. More importantly, even when the same test method was used the variation was unacceptably high. Within a given laboratory, however, the reproducibility was much better and when results were compared for two laboratories that work closely together, the agreement was good. Based on this, the participants in the study concluded that the problem arose because each laboratory used their own methods to fabricate their samples and their own test procedures to run the measurement. The re commendation was made that a follow-up study be conducted to address these questions.

\section{VAMAS Program}

To do just this, a new program was recently initiated under the auspices of the Polymer Composites Working Group of VAMAS, the Versailles Project on Advanced Materials and Standards (Slide 27). This is an international organization that promotes cooperative work on prestandards research. The project on interface testing currently has 17 participating laboratories from 7 countries. It is co-organized by NIST (Donald Hunston) and Michigan State University (Larry Drzal). There are three objectives in the program (Slide 28). First, the participants will develop a recommended test procedure for fabricating the samples and performing the tests. The initial work will be with the fragmentation experiment, but if the effort is successful and there is sufficient interest, other tests can be added in the future. The second objective is for participants to use the recommended test procedure and perform measurements on samples made at a central location with a fixed procedure. The initial comparison of results will focus on the fragment length distribution at saturation so the conclusion will be independent of analysis method. If agreement can't be obtained at this level, then further analysis will not help. In addition, all of the data from the tests will be recorded and samples and material will be made available for other characterization tests so as much information as possible can be obtained. These data will then be made available so different researchers can try their own analysis methods. The final objective seeks to take advantage of the expertise and experience of the participants. Just as in this workshop, the VAMAS program will seek to encourage information exchange among researchers in the area, to provide a forum for identifying the critical research issues in the field, and to encourage cooperation among scientists in the field.

\section{REFERENCES}

1. G. B. McKenna, "Interlaminar Effects in Fiber-Reinforced Plastics-A Review," PolymerPlast. Tech. Eng. 5(1), 23-53 (1975).

2. A. Eitan, and H. D. Wagner, "Fiber Interactions in Two-Dimensional Composites, Appl. 
Phys. Lett. 58(10), 1033-1035 (1991).

3. D. G. Harlow, and S. L. Pheonix, "The Chain-of-Bundles Probability Model for the Strength of Fibrous Materials I: Analysis and Conjecture," J. Composite Mater. 12, 195 (1978) and "The Chain-of-Bundles Probability Model for the Strength of Fibrous Materials II: A Numerical Study of Convergences," J. Composite Mater. 12, 314 (1978).

4. P. J. Herrera-Franco, V. Rao, L. T. Drzal, and M. Y. M. Chiang, "Bond Strength Measurement in Composites--Analysis of Experimental Techniques," Composites Eng. 2(1), 31-45 (1992).

5. C. Marotzke, "A Critical Evaluation of Micro-mechanical Interface Test Methods," pp. 7583 in Proc. Composites Testing and Standardization, ECCM-CTS 2, Hamburg, Germany, September 1994.

6. A. N. Gent, Y. W. Chang, M. Nardin, and J. Schultz, "Mechanics of Fibre Fragmentation," J. Mat. Sci. 31, 1707-1714 (1996).

7. C. Y. Hui, S. L. Phoenix, and L. Kogan, "Analysis of Fragmentation in the Single Filament Composite: Roles of Fiber Strength Distributions and Exclusion Zone Models," J. Mech., Phys. Solids 44(10), 1715-1737 (1996).

8. M.. R. Piggot, "Why Interface Testing using Single Fibre Methods can be Misleading," Composite Sci. Tech. 57(8), 985-990 (1997).

9. A. Pegoretti and A. T. DiBenedetto, "The Measurement of Fracture Energy at Fiber-Matrix Interfaces," pp. 391-394 in Proceedings of the 19th Annual Meeting of the Adhesion Society (Adhesion Society, Blacksburg, 1996).

10. E. M. Asloun, M. Nardin, J. Schultz, "Stress Transfer in Single-Fiber Composites: Effect of Adhesion, Elastic Modulus of Fibre and Matrix, and Polymer Chain Mobility," J. Mat. Sci. 24, 1835-1844 (1989)

11. J. Schultz and M. Nardin, "Some Physico-Chemical Aspects of the Fiber-Matrix Interphase in Composite Materials," pp. $59-71$ in Adhesion International 1993. L. H. Sharp, Ed. (Gordon and Breach, New York, 1993).

12. L. T. Drzal, M. J. Rich, and P. F. Lloyd, "Adhesion of Graphite Fibers to Epoxy Matrix: I. The Role of Fiber Surface Treatment," AFWAL-TR-83-4099, Materials Laboratory, AFWAL, Wright-Patterson Air Force Base, August 1983.

13. C. T. Chou, and L. S. Penn, "Chemical Bonding and Physical Interaction by Attached Chains at the Fiber-Matrix Interface," J. Adhesion 36, 125-137 (1991).

14. R. K. Agrawal, and L. T. Drzal, "Adhesion to Glass: Relationship to Polyurethane Matrix Morphology and Interphase Chemistry," pp. 367-381 in Composites: Meeting Transportation Challenges, Advanced Composites Conference and Exposition (ASM, Metals Park, 1995).

15. P. Herrera-Franco, W. L. Wu, L. T. Drzal, D. L. Hunston, "Comparison of Methods to Assess Fiber-Matrix Interface Strength, pp. 21-23 in Proceedings of the 14th Annual Meeting of the Adhesion Society (Adhesion Society, Blacksburg, 1991).

16. W. G. McDonough, P. J. Herrera-Franco, W. L. Wu, L. T. Drzal, D. L. Hunston, "FiberMatrix Bond Tests in Composite Materials," Proceedings of the 23rd Intl. SAMPE Technical Conf. (SAMPE, Covina, 1991).

17. M. S. Madukar, and L. T. Drzal, "Fiber-Matrix Adhesion and its Effect on Composite Mechanical Properties: I. In Plane and Interlaminar Shear Behavior of Graphite/Epoxy Composites," J. Compos. Mater. 25, 932-957 (1991) and "Fiber-Matrix Adhesion and its Effect on Composite Mechanical Properties: II. Longitudinal $\left(0^{\circ}\right)$ and Transverse $\left(90^{\circ}\right)$ 
Tensile and flexure Behavior of Graphite/Epoxy Composites," J. Compos. Mater. 25, 957 991 (1991).

18. C. R. Schultheisz, C. L. Schutte, W. G. McDonough, K. S. Macturk, M. McAuliffe, S. Kondagunta, D. L. Hunston, "Effect of Temperature and Fiber Coating on the Strength of EGlass Fibers and the E-Glass/Epoxy Interface for Single-Fiber Fragmentation Samples Immersed in Water," pp. 103-131 in ASTM Symposium on Fiber, Matrix and Interface Properties, ASTM STP 1290 (ASTM, West Conshohocken, PA, 1996), and references therein.

19. K. S. Macturk, C. R. Schultheisz, D. L. Hunston, C. L. Schutte, "The Effect of Coupling Agent on Composite Durability," pp. 403-406 in Proceedings of the 19th Annual Meeting of the Adheison Society (Adhesion Society, Blacksburg, 1996).

20. D. L. Hunston, K. S. Macturk, C. R. Schultheisz, G. Holmes, W. G. McDonough, and C. L. Schutte, "The Role of Silane Surface Treatments in Strength and Durability of Fiber-Matrix Bonding in Composites," Vol. 2, pp. 427-432 in Proc. EurAd'96, European Adhesion Conference (The Institute of Materials, London, 1996).

21. C. R. Schultheisz, W. G. McDonough, S. Kondagunta, C. L. Schutte, K. S. Macturk, M. McAuliffe, and D. L. Hunston, "Effects of Moisture on E-Glass/Epoxy Interfacial and Fiber Strengths," in ASTM Symposium on Composite Materials: Testing and Design, ASTM STP 1242 (ASTM, West Conshohocken, PA, in press).

22. L. T. Drzal, M. J. Rich, and F. Koenig, "Adhesion of Graphite Fibers to Epoxy Matrices. III. The Effects of Hygrothermal Exposure," J. Adhesion 18, 49-72 (1985).

23. M. R. Meyer, R. A. Latour, Jr., and H. D. Shutte, "Long-Term Durability of Fiber/Matrix Interfacial Bonding in Hygrothermal Environments," pp. 30-29 in Proc. Amer. Soc.

Composites (Technomics Pub. Co., Lancaster, 1993).

24. M. J. Pitkethy, J. P. Favre, U. Gaur, J. Jakubowski, S. F. Mudrich, D. L. Caldwell, L. T. Drzal, M. Nardin, H. D. Wagner, L. DiLandro, A. Hampe, J. P. Armistead, M. Desaeger, and I. Verpoest, "A Round-Robin Programme on Interfacial Test Methods," Composites Sci. \& Eng. 205-214 (1993).

25. A. Kelly, and W. R. Tyson, "Tensile Properties of Fiber-Reinforced Materials: Copper/Tungsten and Copper/Molybdenum," J. Mech. Phys. Solids 13, 329-350 (1965).

26. A. Argon, and A. Gent, "Effect of Interfacial Bonding on Adhesion," J. Polym Sci, Polym Phys. 13, 1285-1300 (1975). 


\section{PERFORMANCE PROPERTY RELATIONSHIPS}

\section{Donald Hunston}

National Institute of Standards \& Technology

Polymers Division

Bldg. 224, Rm B108

Gaithersburg, MD 20899

Slide 1

\section{PERFORMANCE PROPERTY RELATIONSHIPS}

- MAJOR OBJECTIVE for micro-mechanical tests is to provide information on composite behavior

- CRITICAL QUESTION is the relationship between micro-mechanical test results and composite performance properties 


\section{Three Levels of Comparison}

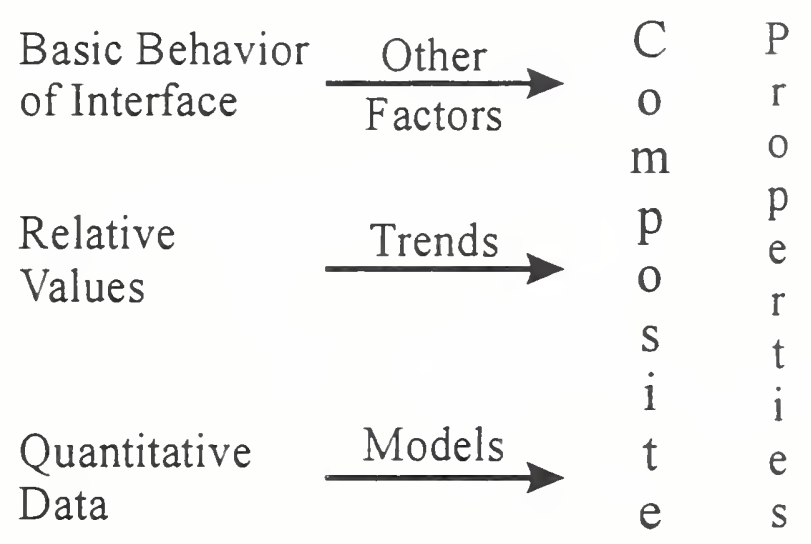

Slide 3

\section{Uncertainties in Micro-Tests}

- How do you do the test correctly

-What does the test measure

„ Failure Mode: Shear vs Tension

»Different Stress States

- Direct comparison - Augment not substitute for improved analysis 


\section{Outline}

- Examples: Compare

» Different Micro-Tests

» Micro- and Macro- Tests

»Durability Tests

- Conclusions

Slide 5

\section{Micro-Test Methods}
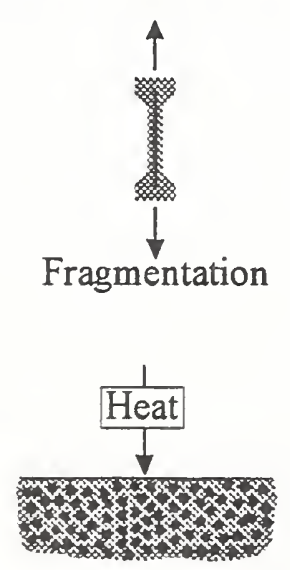

Thermo-Accoustic

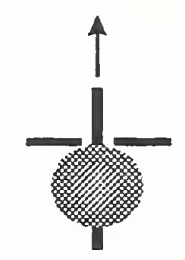

Bead Pull-off

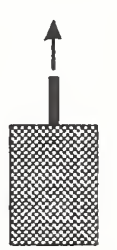

Fiber Pull-out
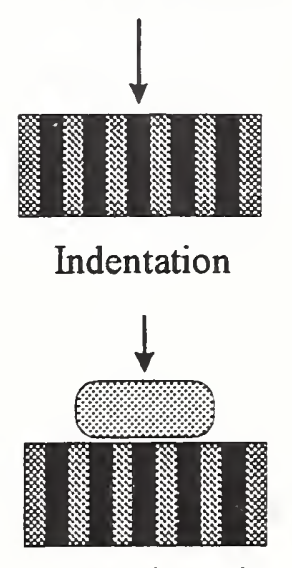

Meso-Indentation

Slide 6 


\section{Test Samples}

Resin: Epoxy - Epon 828 \& mPDA

Fibers: AU-4 - fiber surface untreated

AS-4 - fiber surface treated for bonding

IM6-U - fiber surface untreated

IM6-100 - fiber surface treated for bonding

IM6-600 - fiber surface treated 6 times as long as IM6-100

Slide 7

\section{Results for AS Fibers}

\begin{tabular}{|c|c|c|c|}
\hline \multirow{2}{*}{ Test Method } & $\begin{array}{c}\text { AS-4 } \\
\text { Sample }\end{array}$ & $\begin{array}{c}\text { AU-4 } \\
\text { Sample }\end{array}$ & \multirow{2}{*}{ Ratio } \\
\cline { 2 - 3 } & \multicolumn{2}{|c|}{ Shear Strength (MPa) } & \\
\hline Fragmentation & $68 \pm 6.1$ & $37 \pm 4.1$ & 1.8 \\
\hline Micro-Drop & $50 \pm 3.9$ & $23 \pm 3.7$ & 2.2 \\
\hline Indentation & $71 \pm 12$ & $55 \pm 8.3$ & 1.3 \\
\hline
\end{tabular}

The standard uncertainty in shear strength (taken as one standard deviation) is shown in the Table.

Slide 8 


\section{Results for IM6 Fibers}

\begin{tabular}{|c|c|c|c|c|}
\hline \multirow{2}{*}{ Test Method } & IM6-600 & IM6-100 & IM6-U & \multirow{2}{*}{ Range } \\
\hline & \multicolumn{3}{|c|}{ Shear Strength (MPa) } & \\
\hline Fragmentation & $47 \pm 4.7$ & $40 \pm 4.0$ & $22 \pm 2.2$ & 2.1 \\
\hline Micro-Drop & $15 \pm 1.5$ & $19 \pm 1.9$ & $15 \pm 1.5$ & 1.3 \\
\hline Indentation & $43 \pm 11$ & $37 \pm 11$ & $27 \pm 5.0$ & 2.6 \\
\hline
\end{tabular}

The standard uncertainty in shear strength (taken as one standard deviation) is shown in the Table.

Slide 9

\section{Problems}

- Processing challenge - Micro-samples must be the same as composite

»Resin cure state

»Sizing, Wetting, etc. - interface region

»Other?

- Each test has limitations

»Multiple tests desirable

Slide 10 


\section{Macro-Tests Methods}

Compare with results from composite tests

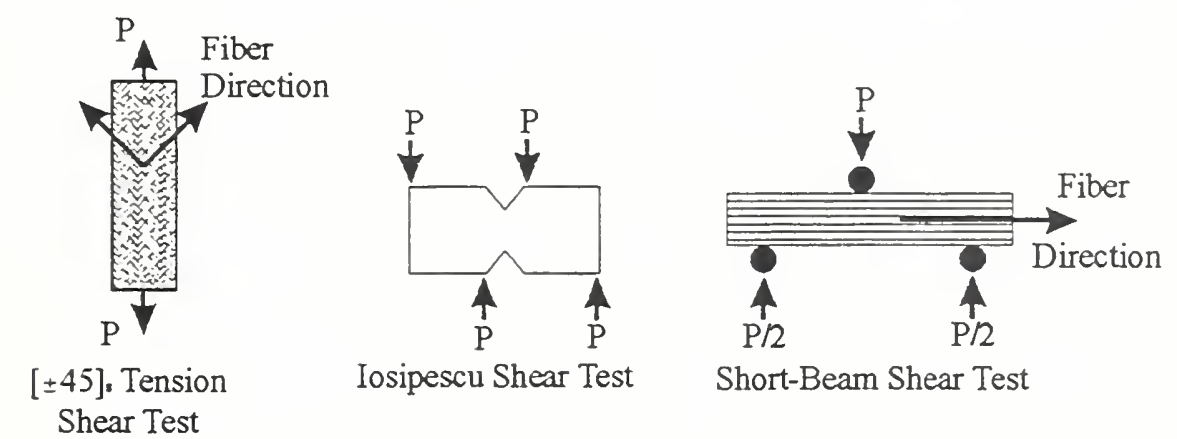

M. S. Madukar and L. T. Drzal, J. Composite Materials, Ref 17.

Slide 11

\section{Comparison with Macro-Tests}

\begin{tabular}{|c|c|c|c|}
\hline \multirow{2}{*}{ Test Method } & \multicolumn{1}{|c|}{$\begin{array}{c}\text { AS-4 } \\
\text { Sample }\end{array}$} & $\begin{array}{c}\text { AU-4 } \\
\text { Sample }\end{array}$ & \multirow{2}{*}{ Ratio } \\
\cline { 2 - 4 } & \multicolumn{2}{|c|}{ Shear Strength (MPa) } & \\
\hline Fragmentation & $68 \pm 6.1$ & $37 \pm 4.1$ & 1.8 \\
\hline Micro-Drop & $50 \pm 3.9$ & $23 \pm 3.7$ & 2.2 \\
\hline Indentation & $71 \pm 12$ & $55 \pm 8.3$ & 1.3 \\
\hline [ \pm 45$]$ s Tension & $72 \pm 12$ & $37 \pm 1.8$ & 1.9 \\
\hline Iosipescu & $96 \pm 5.1$ & $55 \pm 3.0$ & 1.7 \\
\hline Short Beam Shear & $84 \pm 7.0$ & $48 \pm 5.4$ & 1.8 \\
\hline
\end{tabular}

The standard uncertainty in the shear strength (taken as one standard deviation) is shown in Table. 


\section{Durability Studies}

Use fragmentation test to study behavior as a function of time in air and water

Test temperature: $25^{\circ} \mathrm{C}$ and $75^{\circ} \mathrm{C}$

Resin - Epoxy, Epon 828 / mPDA

Fiber - E-Glass

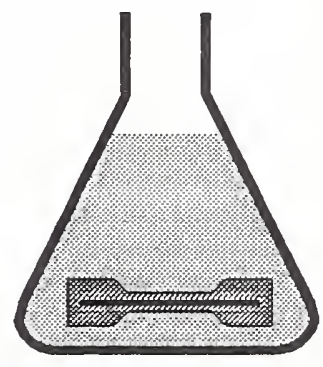

Slide 13

\section{Data Analysis}

- Low stress data analyzed for fiber strength

$P_{s}(\sigma, L)=\exp \left[\frac{-L}{L_{0}}\left(\frac{\sigma}{\alpha_{0}}\right)^{\beta}\right]$

- Combined with high stress data for interface strength

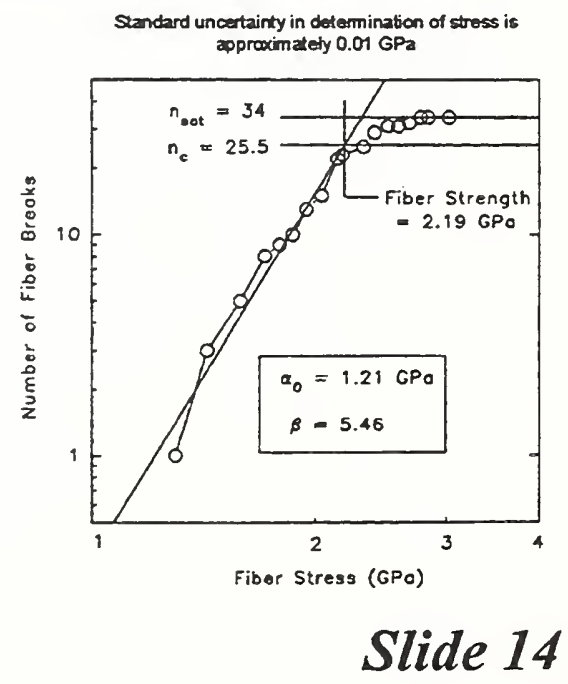




\section{Silane Coupling Agent Study}

- Study effects of coupling agents on durability in $75^{\circ} \mathrm{C}$ water

- Two coupling agents used individually or in 65-35 mixture

- APS can bond to epoxy, OTS cannot

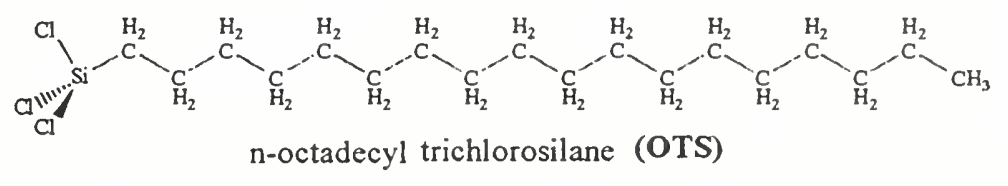

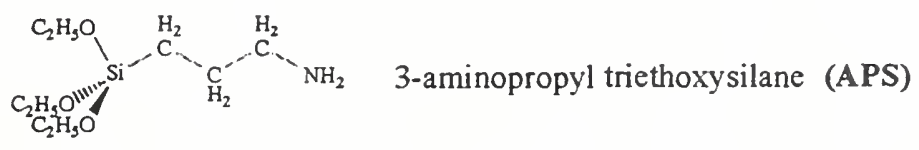

Slide 15

\section{Dry Strength Data}

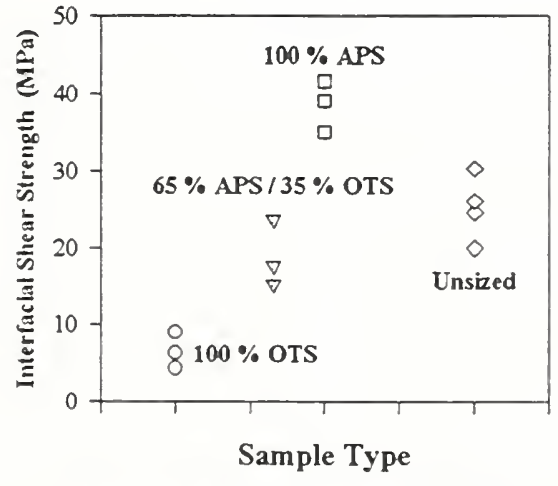

The standard uncertainty in the shear strength results (taken as one standard doviation) is approcimately $\pm 3.2 \mathrm{MP2}$.
- Increased chemical bonding improves adhesion

- Dry glass has good adhesion

- Results show exactly same trend as flat plate studies

Slide 16 


\section{Durability Results}

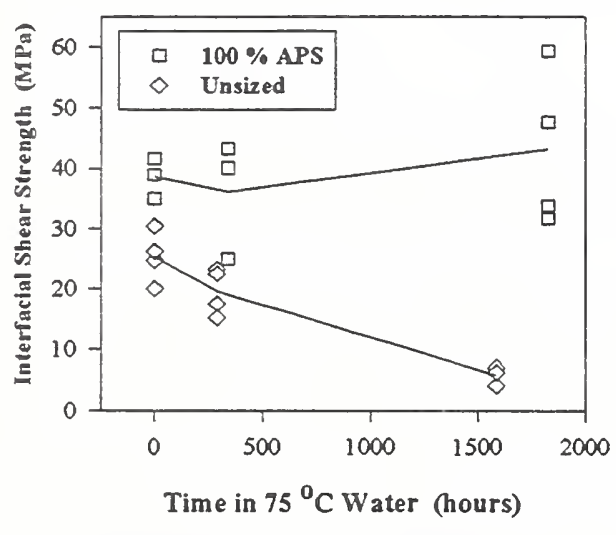

The standard uncertainty is shear strength data (taken as one dard deviation) is approdimately +32 MP for smples not exposed

to deviton) is approsma dy $+32 \mathrm{MP}$ a for smplos not exposect uncertainty (taken as one standard deviation) is approximately $30 \%$.
- Glass-epoxy interface attacked by moisture

- Chemical bonding improves durability

- Results show exactly same trend as flat plate studies

Slide 17

\section{Comparison with Composites}

- Compare Fragmentation and Composite Results

- E-glass fibers - Epoxy and vinyl ester compatible sizings

- Two Temperatures: $25^{\circ} \mathrm{C}$ and $75^{\circ} \mathrm{C}$

FIBER

STRENGTH

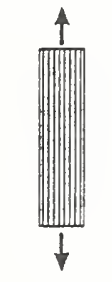

Tension
INTERFACE STRENGTH

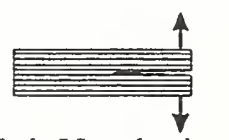

Mode I Interlaminar Fracture

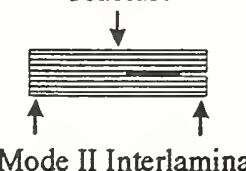

Fracture

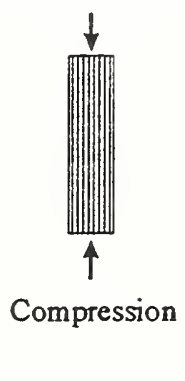

Slide 18 


\section{Fiber \& Tensile Strength}

\section{Fragmentation}

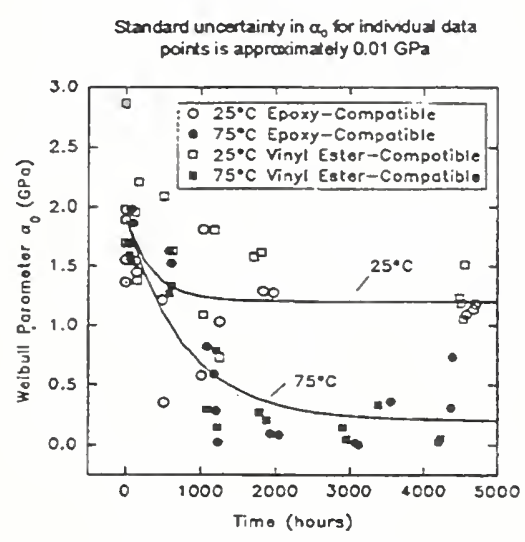

Composite

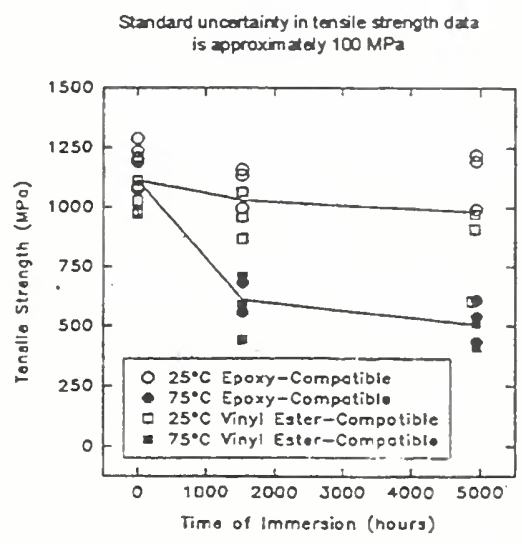

Slide 19

\section{Model Predictions}

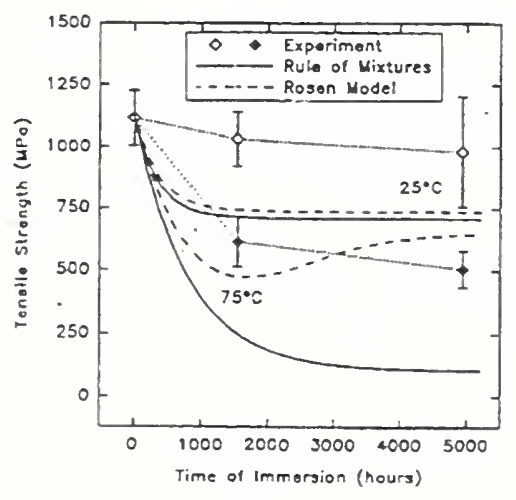

Error bars represent standard uncertainty in the data (taken as one standard deviation)
- Simple models predict general trends

- Simple models overestimate the strength reduction

- Better analysis needed for both test 


\section{Fragmentation Results}

- Degradation

observed at both temperatures with both coatings

- Biggest effect with vinylester comp. coating at $75^{\circ} \mathrm{C}$

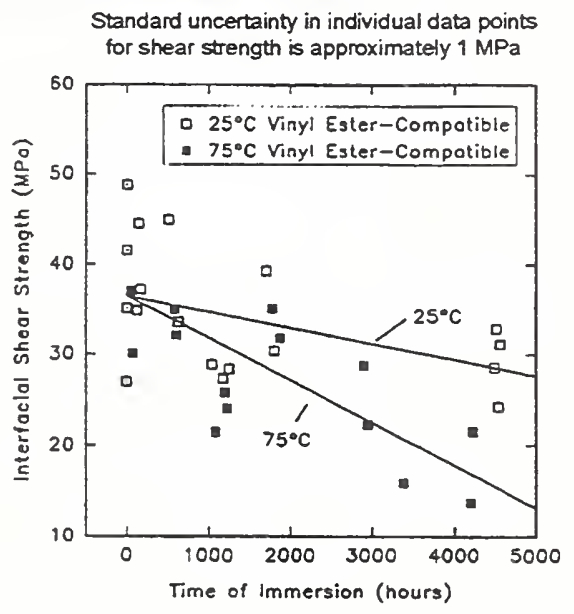

Slide 21

\section{Mode I Interlaminar Fracture}

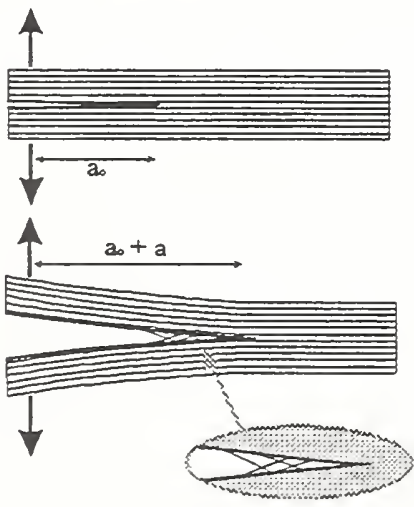

Fiber Bridging

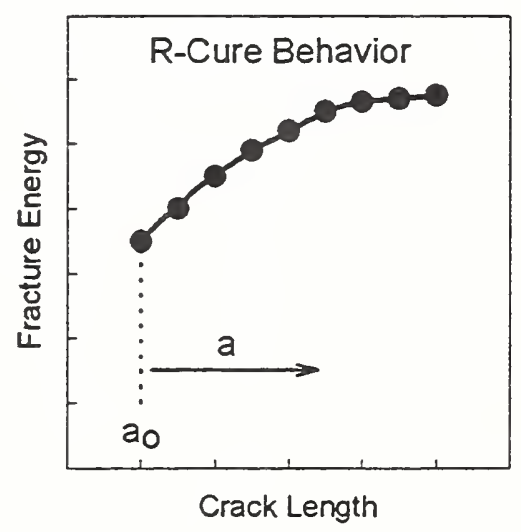

Slide 22 


\section{Interlaminar Fracture Results}

Epoxy Compatible Vinylester Compatible
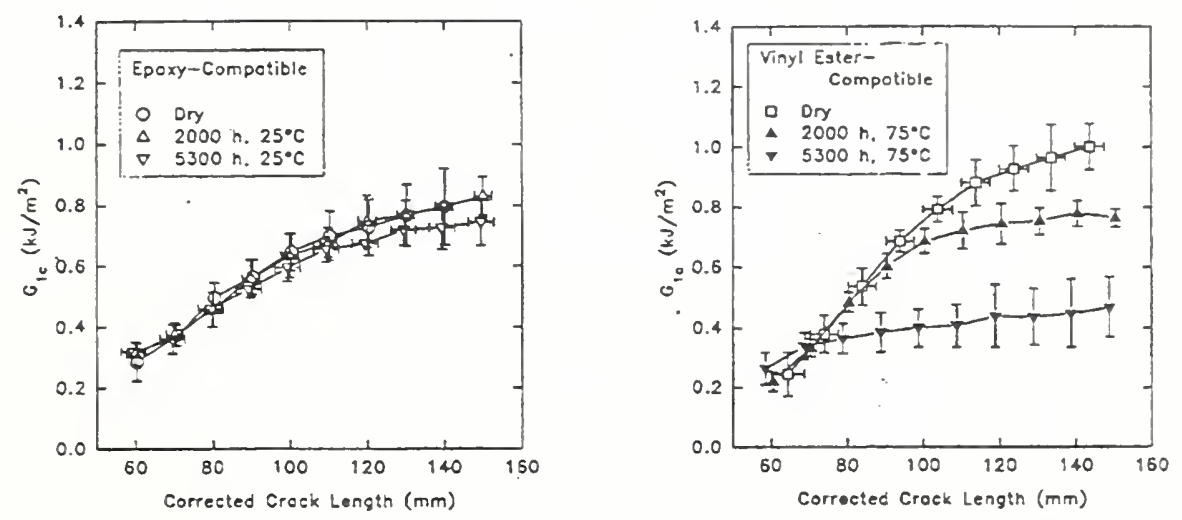

Error bars represent standard uncertainty in the data (taken as one standard deviation)

Slide 23

\section{Conclusions}

- Correlations can be useful

- Relative values can show useful trends, absolute values seldom agree

- Each test method has limitations multiple test methods useful

- Correlations don't always work, at present 


\section{Conclusions}

- Problems

„Processing differences

„Comparison Problems

-What do micro-tests really measure

- How do we translate this to composites

- Needs:

" Improved analysis - Micro and Macro tests

»Standardization of test methods

Slide 25

\section{Round Robin Results}

Pitkethly, et. al., Composites Science and Technology 44, 205 (1993).

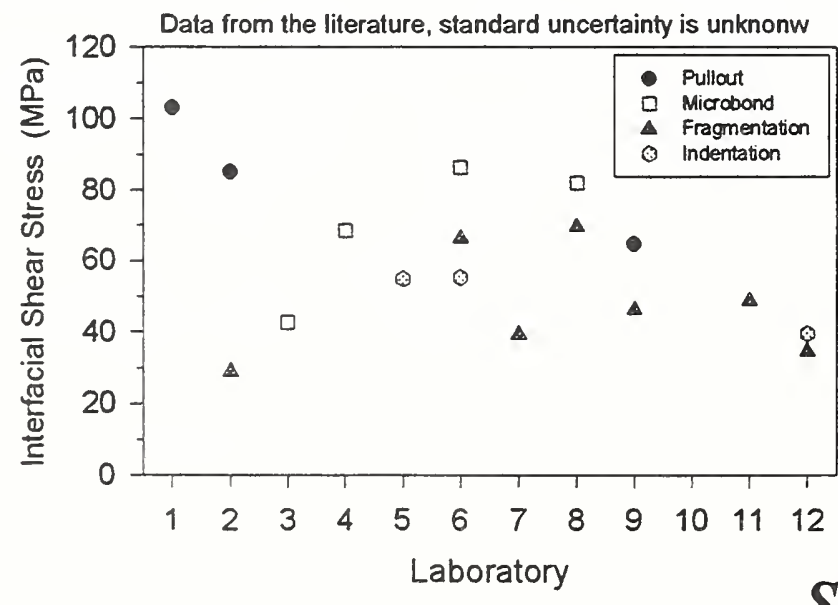

Slide 26 


\section{New Round Robin}

- Under auspices of Polymer Composites Working Group of VAMAS ${ }^{1}$

- International participation (17 laboratories)

- Co-organized with Larry Drzal

${ }^{1}$ Versailles Project on Advanced Materials and Standards

Slide 27

\section{Objectives}

- Develop a recommended test procedure

- Conduct tests with a single batch of samples (fragmentation)

- Provide forum for identifying critical research issues

Slide 28 



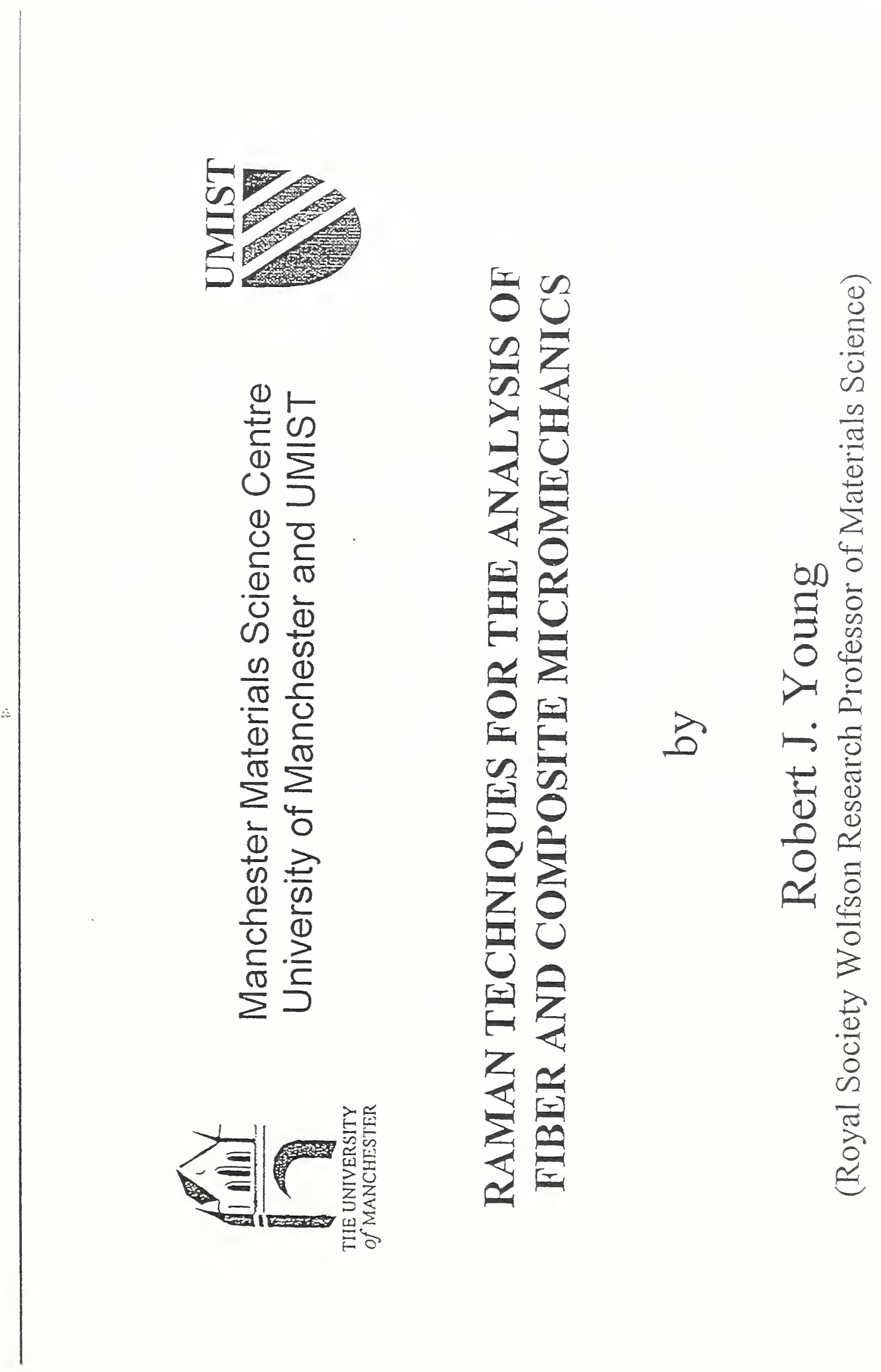


Slide 2. Programme has been developing since 1982.

Slide 4. Raman microscopy is used for a wide range of materials. Fluorescence microscopy is only possible with $\alpha-\mathrm{AL}_{2} \mathrm{O}_{3}$

Slide 5. Lay-out of Renishaw Raman Imaging Microscopy. It can be used in both a spot mode (microprobe) and an imaging mode.

Slide 6. Schematic lay-out of the Renishaw instrument in the micro probe mode.

Slide 7. Aramid fibres such as Kevlar and Twaron give very well-defined Raman spectra.

Slide 8. Aramid band shift is linear with stress.

Slide 10. The fibre is fixed to the beam with PMMA cement. The strain is uniform along the fibre in the 4-point bend geometry. The diagram shows the fibre in axial compression. If it is inverted the fibre can be deformed in tension.

Slide 11. The $1610 \mathrm{~cm}^{-1}$ aramid band shifts to lower wavenumbers in compression and to higher wavenumbers in tension.

Slide 12(a) Count of kink bands using optical microscopy.

(b) Band shift in compression.

Slide 13. Full Raman spectra for a number of different carbon fibres obtained under similar conditions. The spectra becomes more well-defined for the higher modulus fibres.

Slide 14. The second-order band at $2660 \mathrm{~cm}^{-1}$ shifts to lower wavenumbers in tension (+ve) and higher wavenumbers in compression (-ve).

Slide 15. The shift of the $2660 \mathrm{~cm}^{-1}$ band is approximately linear with stress for the T50 carbon fibre.

Slide 16. Schematic diagrams of the test-pieces used in composite micromechanics.

Slide 17. Schematic diagram of the single-fibre pull out test performed in conjunction with Raman microscopy.

Slide 18. Strain distributions determined from Raman band shifts for the pull out of a Kevlar 49 fibre from an epoxy resin block at different levels of applied fibre strain. (The fibre enters the block at $\mathrm{x}=0$ ).

Slide 20. Theoretical analysis of the single-fibre pull-out test using a shear-lag approach with frictions in the deformed region. 
Slide 21. Schematic diagram of the microbond test rig for use in conjunction with the Raman microprobe.

Slide 22. Variation of fibre strain with position along the droplet for the pull-out of an HM40 fibre from an epoxy droplet ( $100 \mu \mathrm{m}$ diameter). (The fibre enters the droplet at $\mathrm{X}=0)$.

Slide 23. Interfacial shear stress distribution derived using the force balance equilibrium for the microbond data in the previous figure.

Slide 24. Variation of the maximum interfacial shear stress with applied fibre strain (outside the droplet) for the pull out of untreated (HM-40) and surface treated (HM-40) carbon fibres from epoxy droplets.

Slide 25. Schematic diagram of the experimental set up for conducting fragmentation tests under a Raman microprobe.

Slide 26. Distribution of fibre stress at different levels of matrix strain during a fragmentation experiment, before fragmentation takes place. The stress was determined from the Raman band shifts.

Slide 27. Variations of interfacial shear stress determined from the fibre stress distributions in the previous figure, using the force balance equilibrium.

Slide 28. Distribution of fibre stress following the first fibre break at the matric strain $\mathrm{e}_{\mathrm{m}}=0.8 \%$ for the fragmentation specimen.

Slide 29. Distribution of fibre stress at different levels of matrix strain during a fragmentation experiment before fragmentation takes places. The stress was determined from the Raman band shifts for the surface treated fibres.

Slide 30. Variations of interfacial shear stress with applied matrix strain during a fragmentation experiment for untreated and surface treated T50 carbon fibres.

Slide 31. The $2660 \mathrm{~cm}^{-1}$ band shifts to lower wavenumbers in tension.

Slide 32. Comparison of the Raman spectra for an HMS-40 carbon fibre in air and in an epoxy resin matrix. The second-order $2660 \mathrm{~cm}^{-1}$ band is well separated from Raman bands of the epoxy resin.

Slide 33. This next section is concerned with the effect of broken fibres upon neighboring fibres. Under stress it is expected that this will cause a stress concentration and Raman microscopy is used to evaluate the behavior. 
Slide 34. Stress distributions in a series of 5 fibres in a planar array in an epoxy matrix $(0=$ fibre diameter). The break in fibre 3 causes a stress concentration in neighboring fibres.

Slide 35. Same specimen as in the previous figure but with the matrix strain increased to $1.1 \%$. All five fibres have fragmented and the breaks line up for these closelyspaced fibres.

Slide 36. Dependence of stress concentration factor (scf) upon inter-fibre separation for adjacent HMS-40 carbon fibres. The experimented data are from the Raman band shifts and the other curves were derived theoretically (workers indicated).

Slide 37. Specimen used to determine stress distributions in arrays of fibres bridging a crack in an epoxy compact tension specimen.

Slide 38. Different Twaron aramid fibres investigated in the crack-bridging experiment (fibres supplied by Akzo Nobel).

Slide 39. Experimental and theoretical strain distributions for aramid fibres bridging a crack (held open) in the epoxy compact tension specimen.

Slide 40. Schematic diagram of the strain distribution in a crack-bridging fibre when the crack is closed.

Slide 41. Experimental data showing reverse sliding during crack closure for an aramid/epoxy specimen.

Slide 42. Schematic illustration of theoretical analysis of crack bridging showing unloading and reloading.

Slide 43. Experimental demonstration of behavior on reloading for a fibre bridging a crack in an aramid/epoxy specimen.

Slide 44. Experimental data showing crack bridging for an array of six fibres. One fibre is ahead of the crack tip and the other five are bridging the crack.

Slide 45. Detailed strain profiles showing the propagation of debonding for an untreated aramid fibre bridging a crack in an epoxy resin specimen.

Slide 46. Scanning electron micrographs of Kevlar plain woven fabric before impregnation with an epoxy resin.

Slide 47. Detailed strain distributions for the epoxy aramid plain weave composite deformed in the horizontal direction at two levels of overall composites strain. 
Slide 48. Detailed strain distribution in 1 cell of the aramid/epoxy plain weave composite subjected to an average strain of $0.2 \%$.

Slide 49. Summary of the uses of Raman spectroscopy to follow deformation micromechanics in high-performance fibres and composites. 


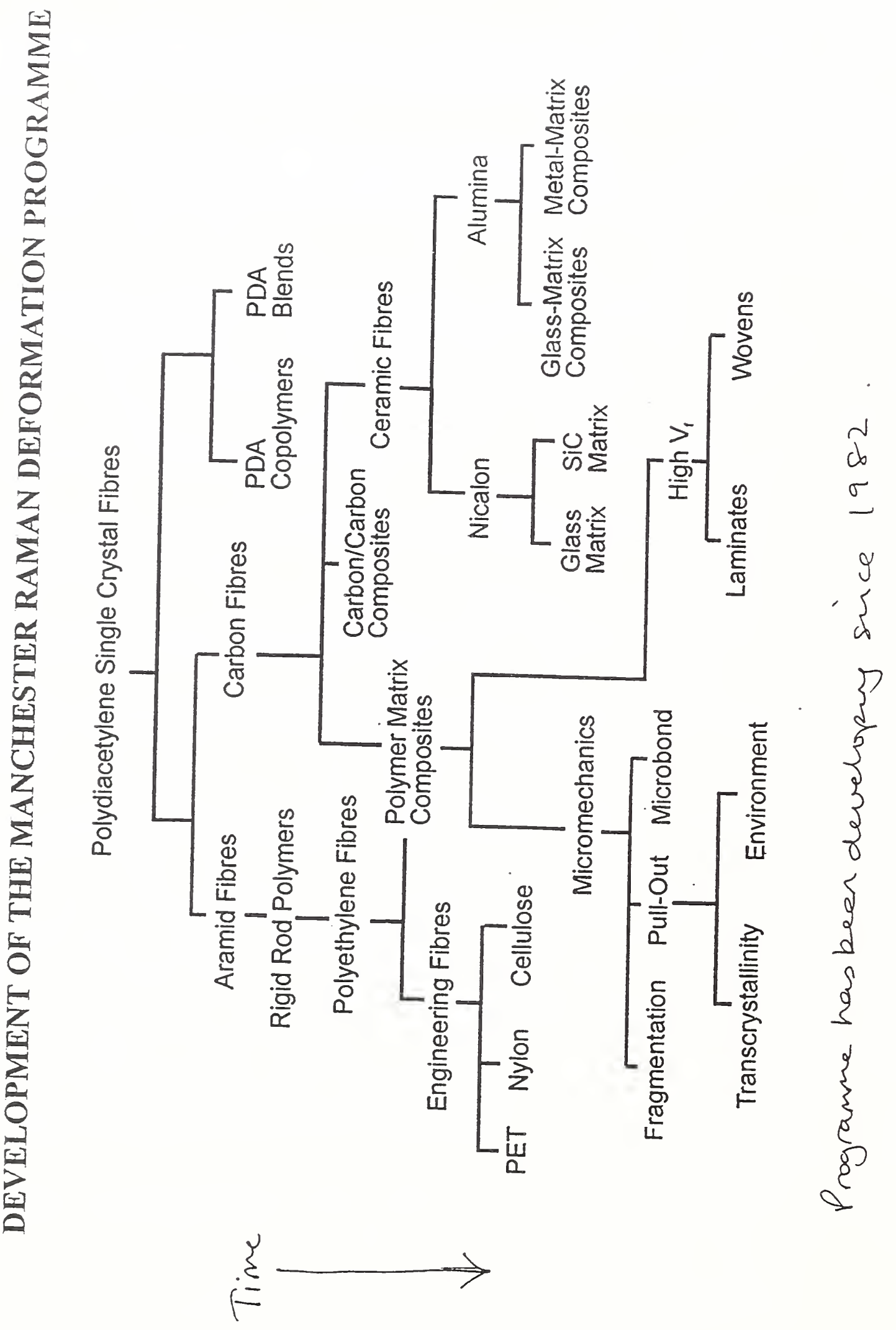

Slide 2. 


\section{CONTENTS}

- RAMAN SPECTROSCOPY

- FIBRE DEFORMATION

- MICROMECHANICAL TEST METHODS

- COMPLEX SYSTEMS

- ADVANTAGES/LIMITATIONS

\section{CO-WORKERS}

MIKE ANDREWS,DAMIAN BANNISTER, RAHMAN YALLEE, YANLING HUANG JIM BENNETT, MARY HEPPENSTALL-BUTLER TONY CERVENKA, JAN MAHY ETC

\section{SPONSORS}

ENGINEERING AND PHYSICAL SCIENCES RESEARCH COUNCIL ROYAL SOCIETY

SHELL RESEARCH, AKZO NOBEL RESEARCH 


\section{RAMAN AND FLUORESCENCE MICROSCOPY}

RAMAN

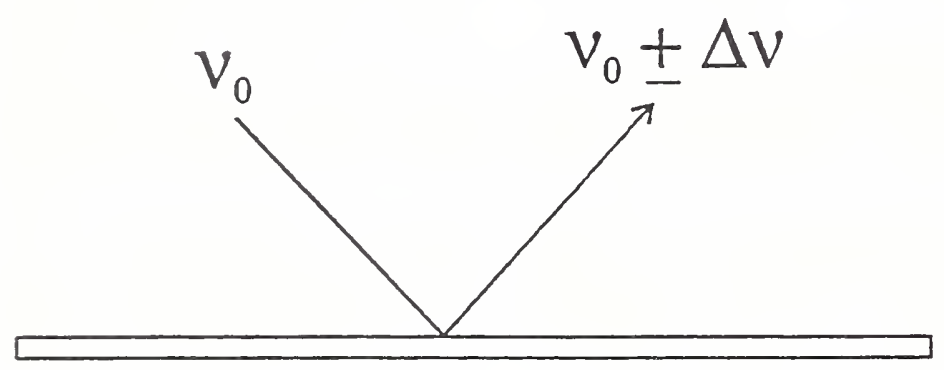

(Atomic/Molecular Vibrations)

FLUORESCENCE

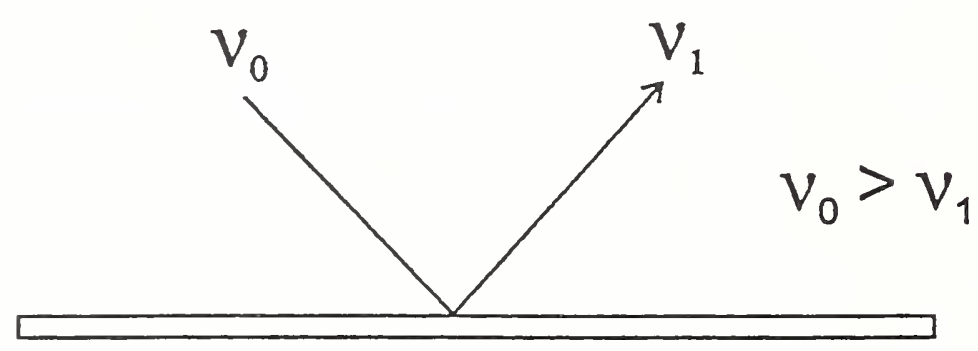

(Electronic Transitions)

N.B. BOTH ARE EXCITED USING VISIBLE LASER LIGHT FOCUSED TO A SPOT IN AN OPTICAL MICROSCOPE Ranan micrascory is used for a uide range of materab Fhorescence microscopy is only prosible whth $\alpha-A_{2} \mathrm{O}_{3}$ 


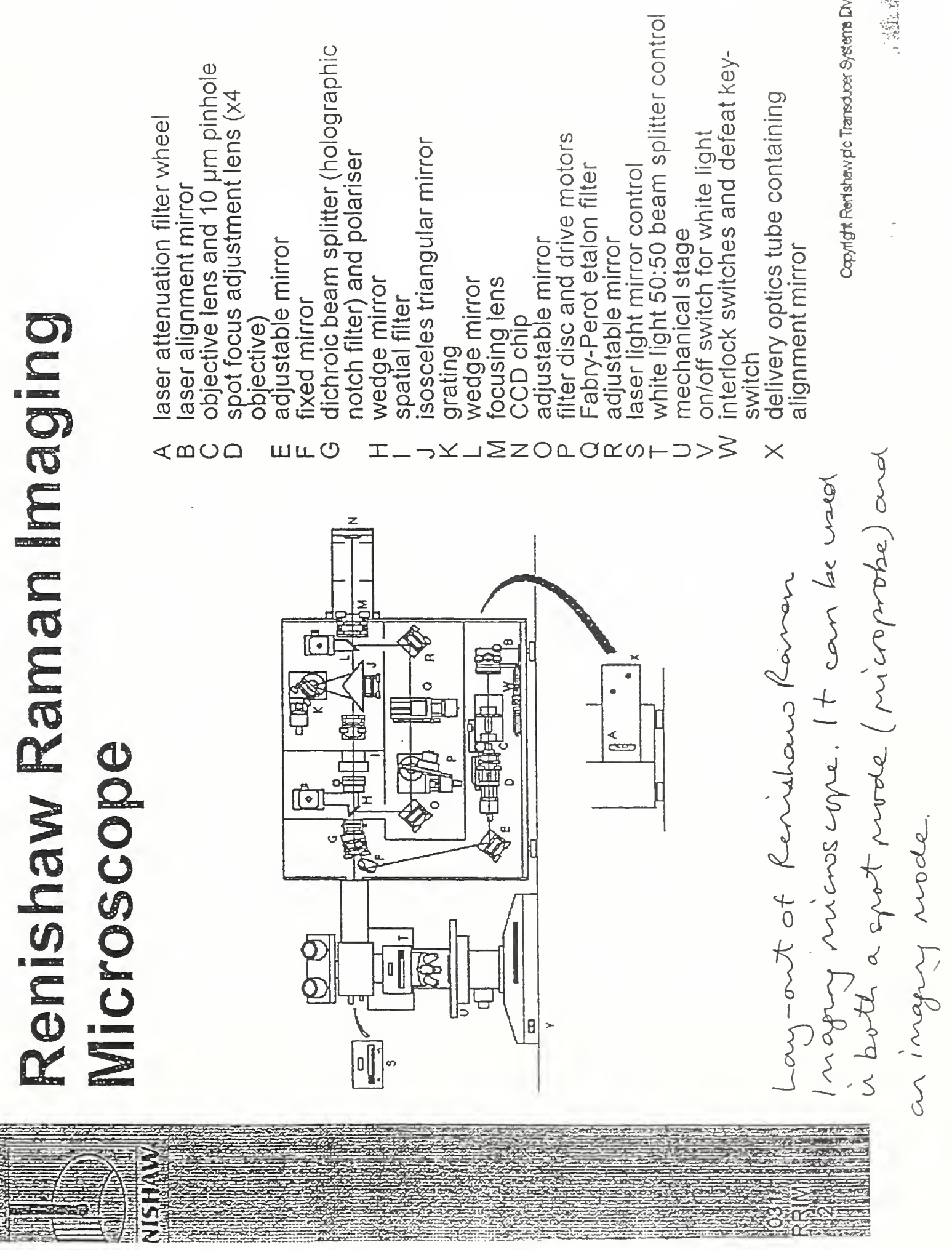

Slide 5 . 


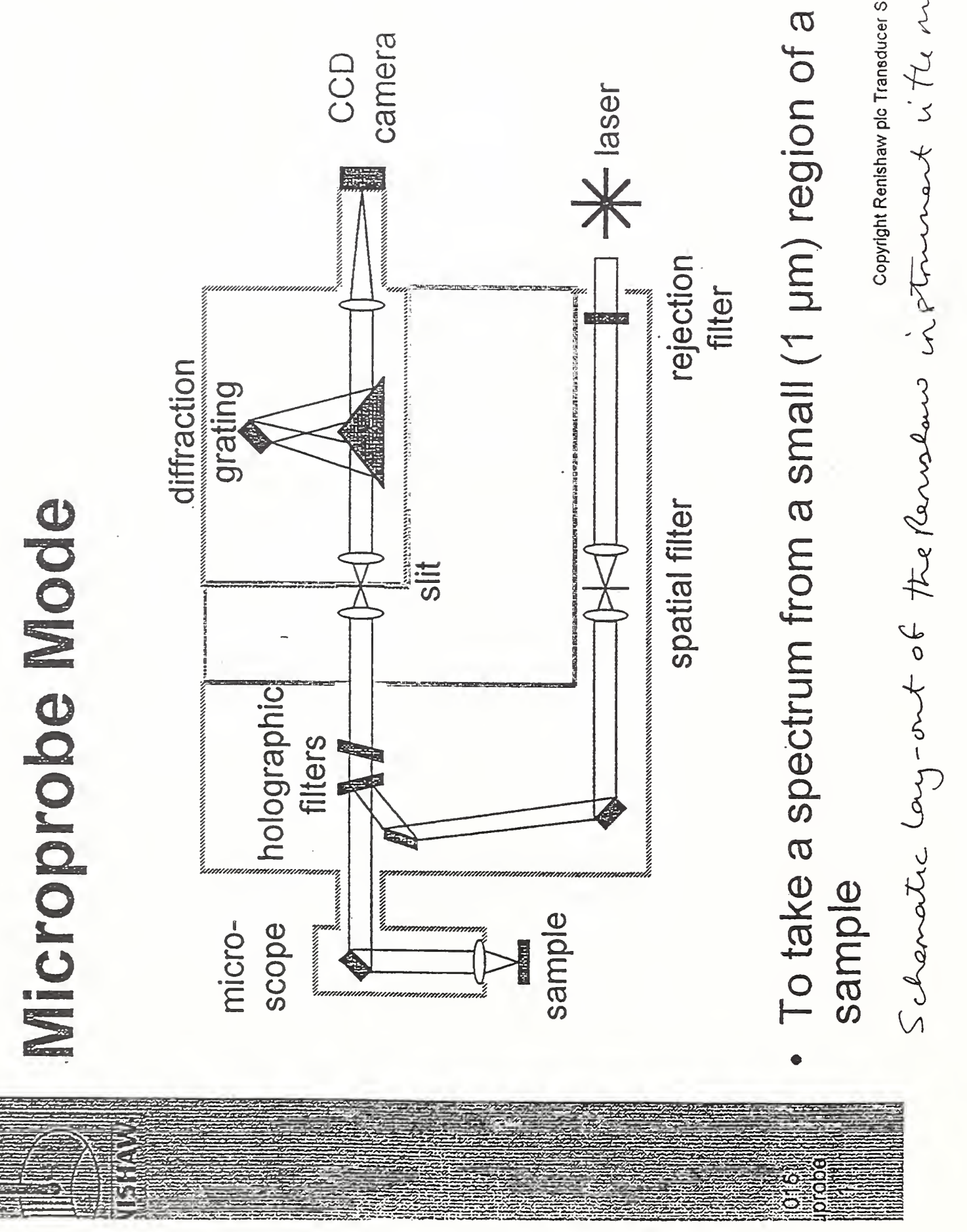

Slide 6 . 


\section{ARAMID FIBRE - TWARON}

$$
\text { Poly(p-phenylene terephthalamide) }
$$

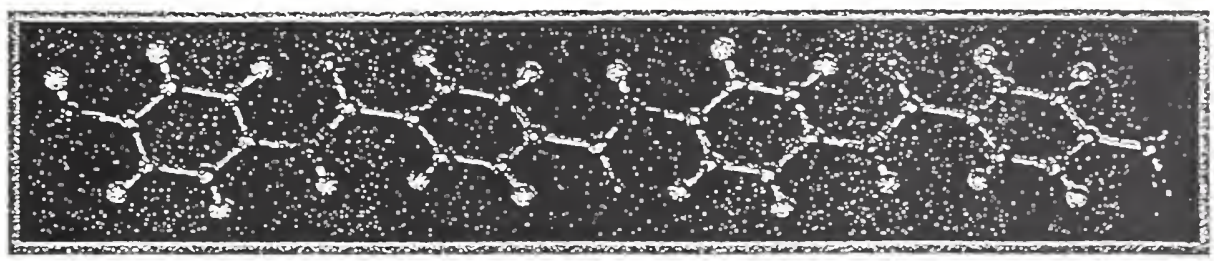

- Young's Modulus - $110 \mathrm{GPa}$

- Fracture Strength - $2.5 \mathrm{GPa}$
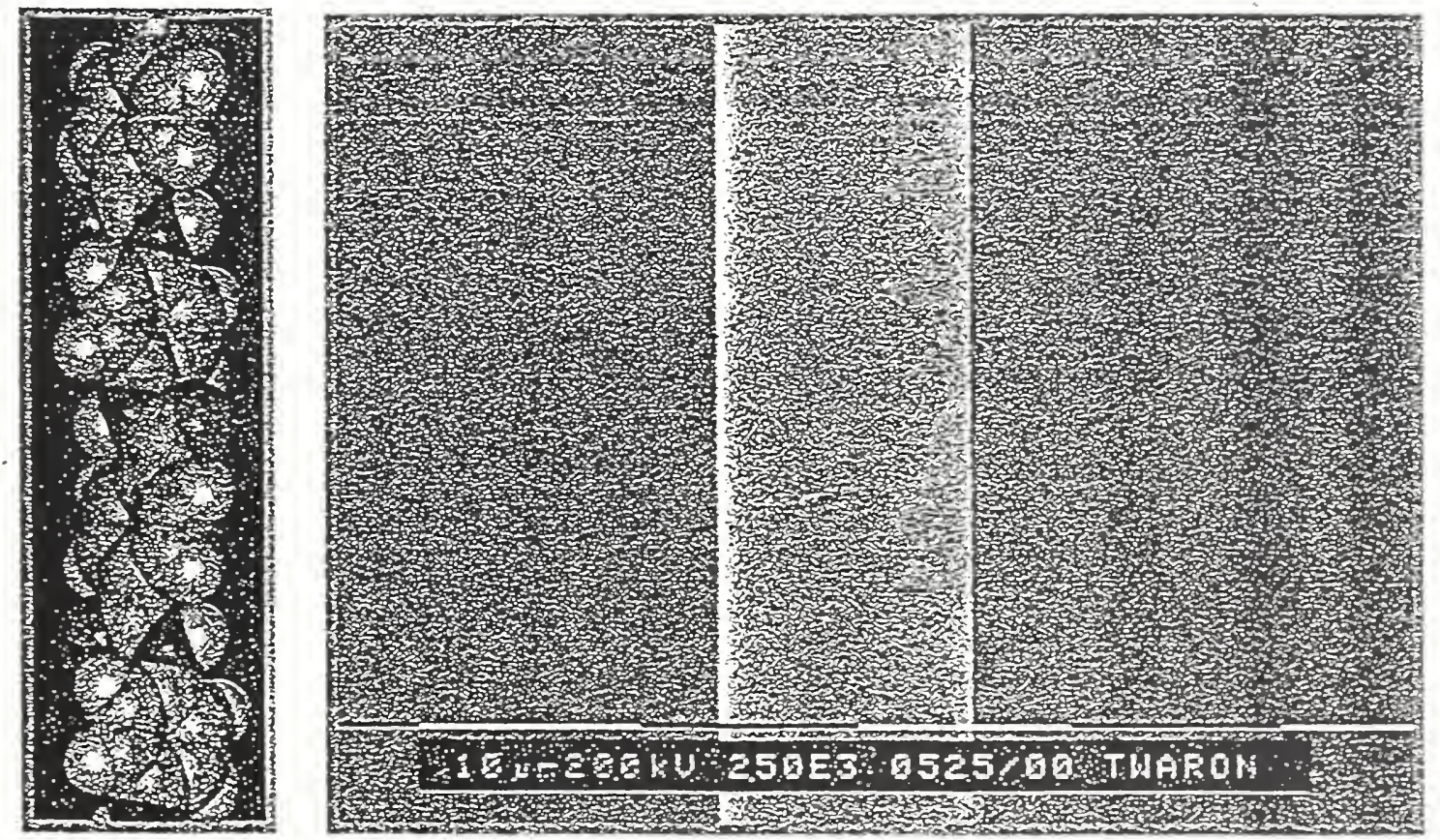

The molecules are highly aligned parallel to the fibre axis

Pranid fibres such as Kevlar and Twasn give very well-dehred Raman spectia. 


\section{MOLECULAR DEFORMATION TWARON}
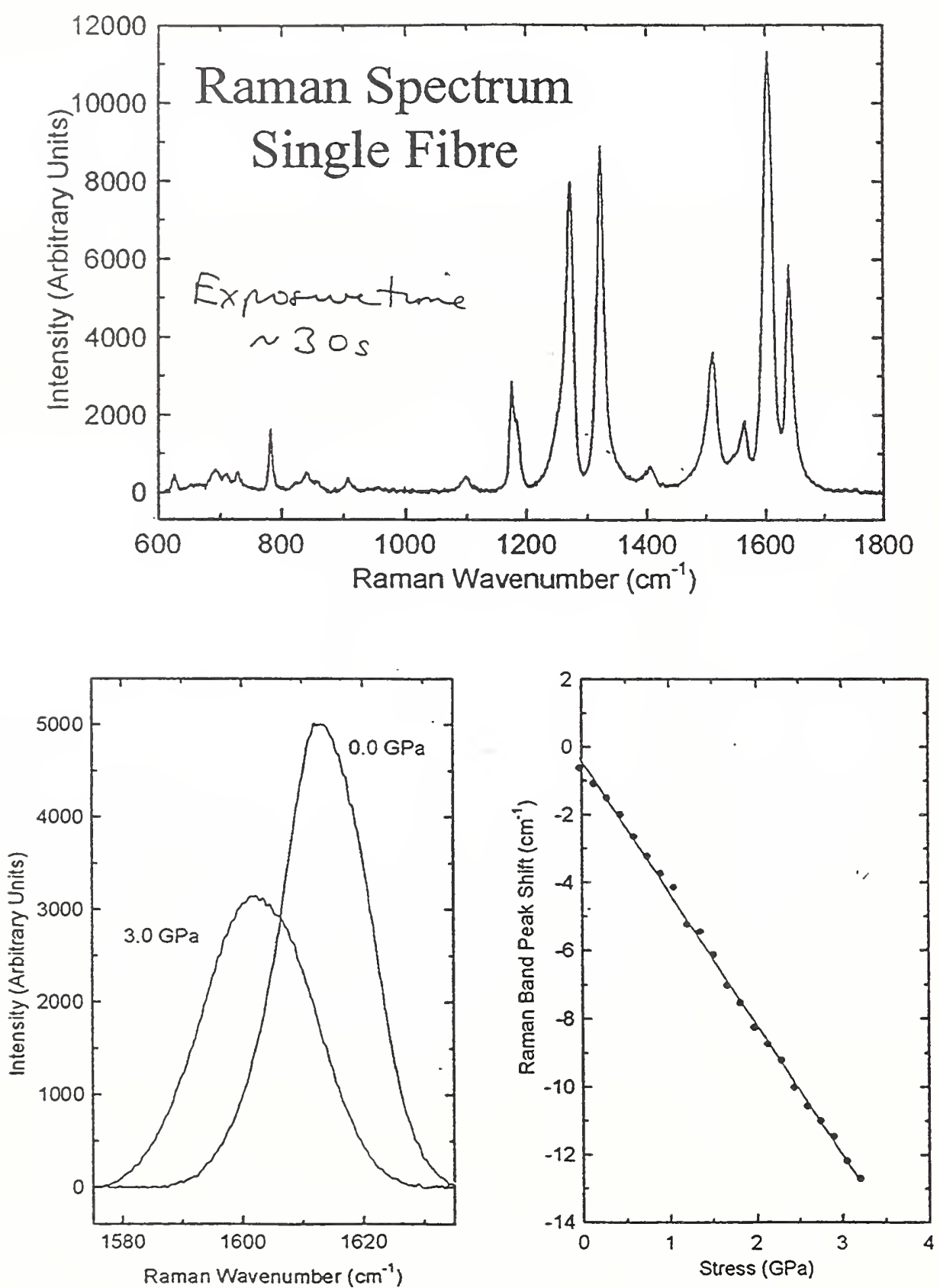

Aranid Band shift is hirear unth stress 


\section{STRESS-INDUCED RAMAN BAND SHIFTS IN ARAMID FIBRES}

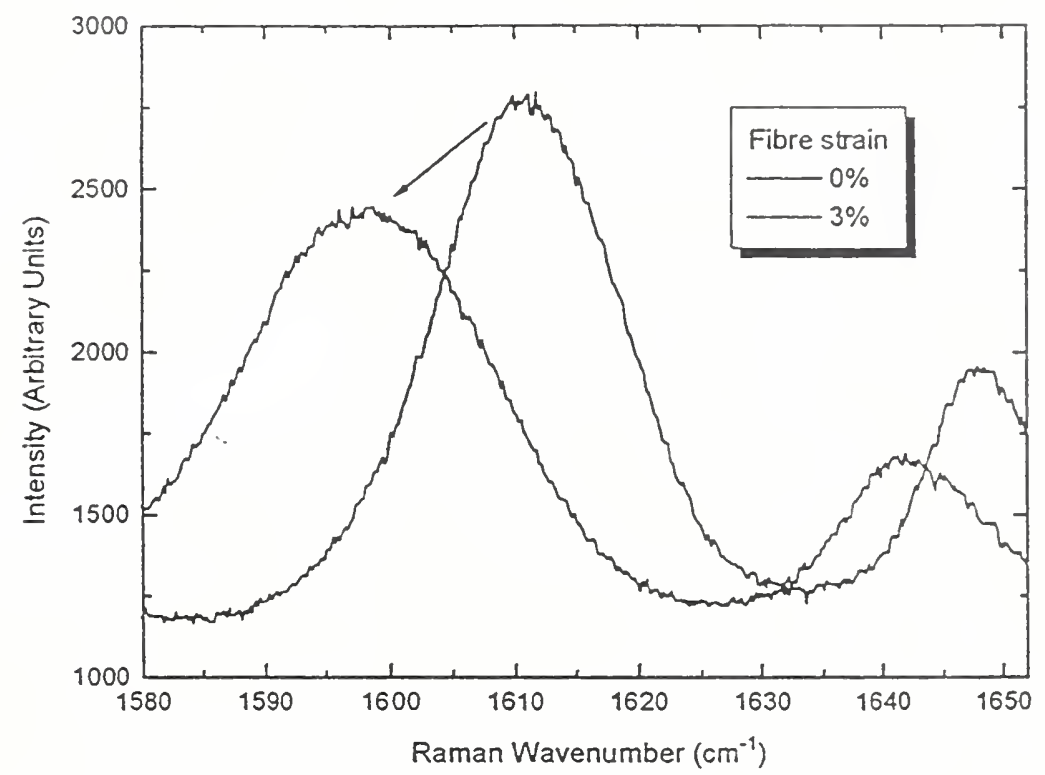

The band at $1610 \mathrm{~cm}^{-1}$ is assigned to the symmetrical vibration of the p-phenylene groups in the aramid molecule

AXIAL FIBRE DEFORMATION

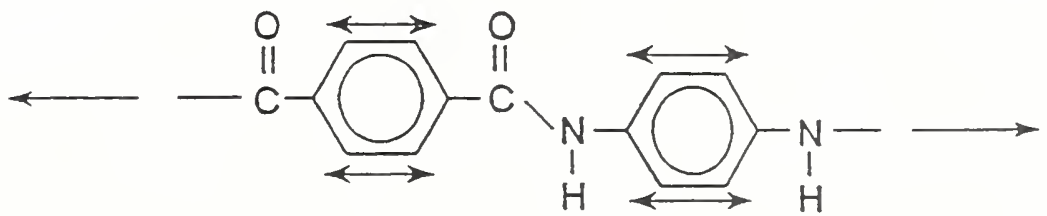

RATE OF BAND SHIFT $-4.0 \mathrm{~cm}^{-1} / \mathrm{GPa}$

(Independent of Fibre Structure)

N.B. THIS IS A UNIVERSAL CALIBRATION FOR ALL AROMATIC POLYMER FIBRES 

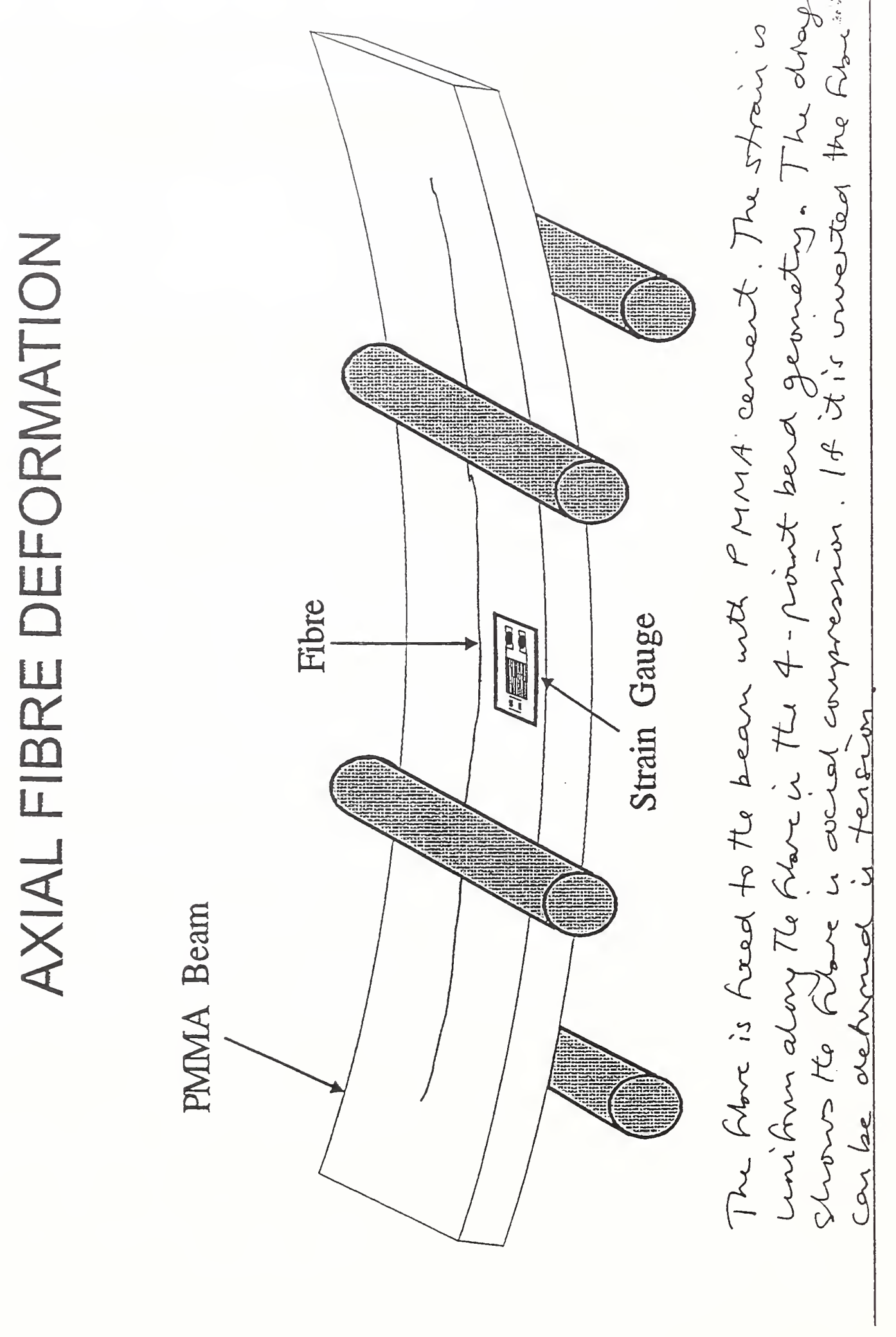

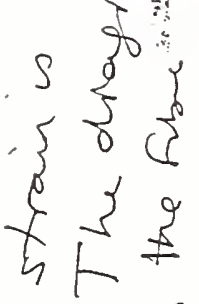

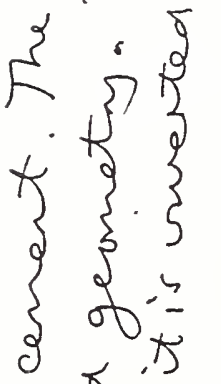

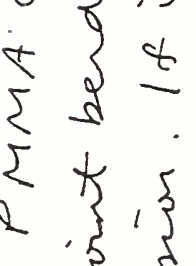

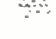

Slide 10. 


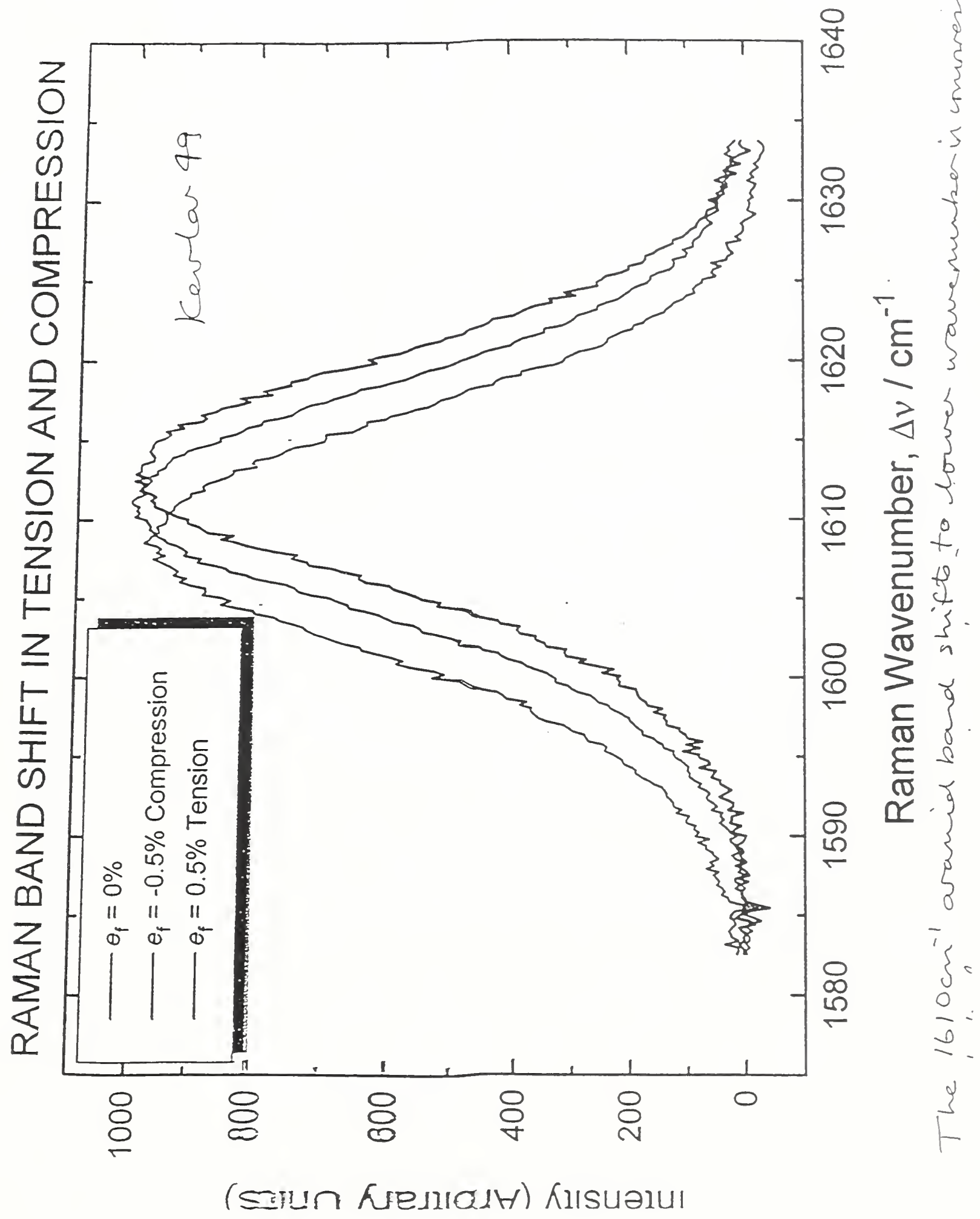

Slide 11. 


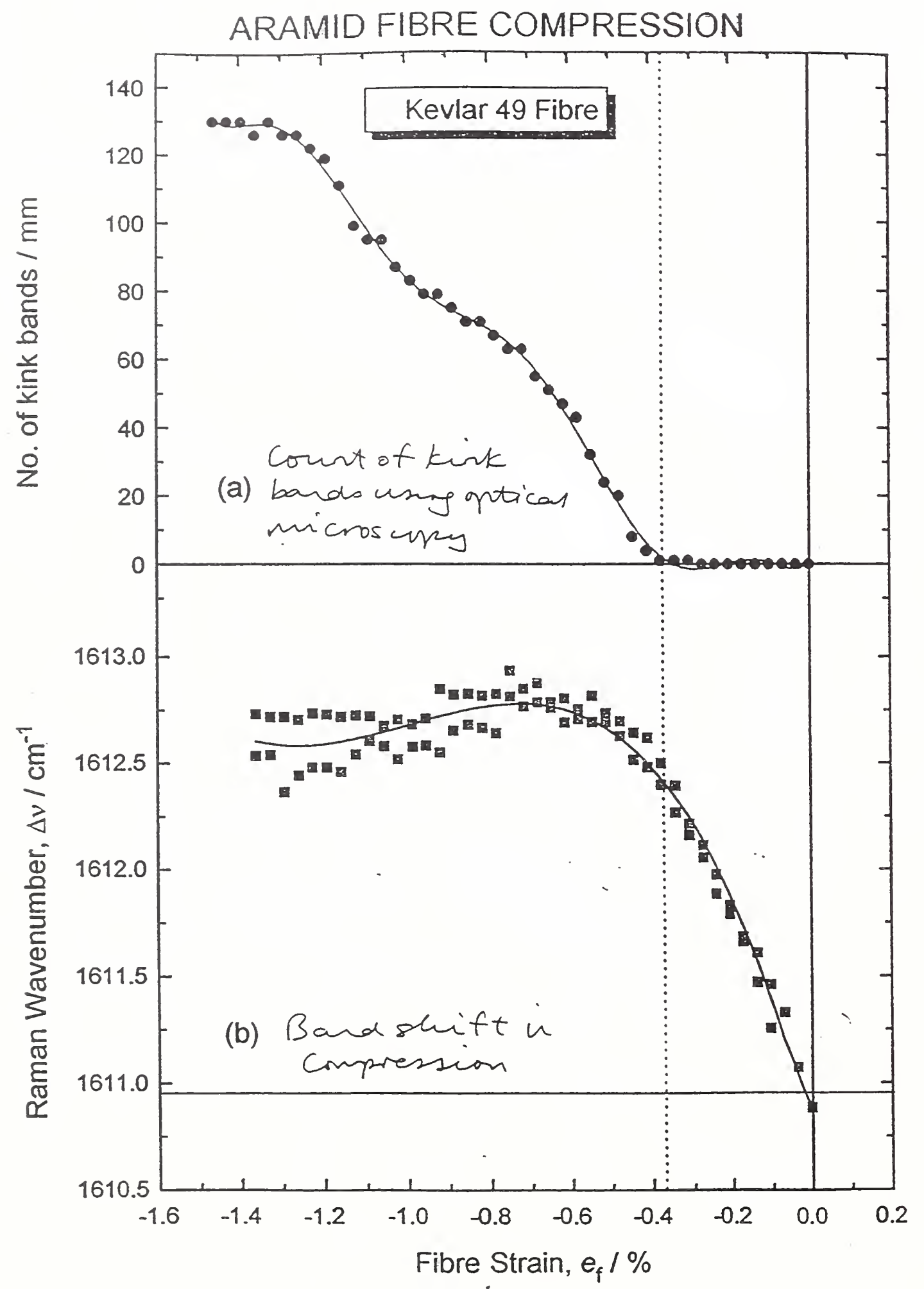

Slide $12(a) \&(b)$ 


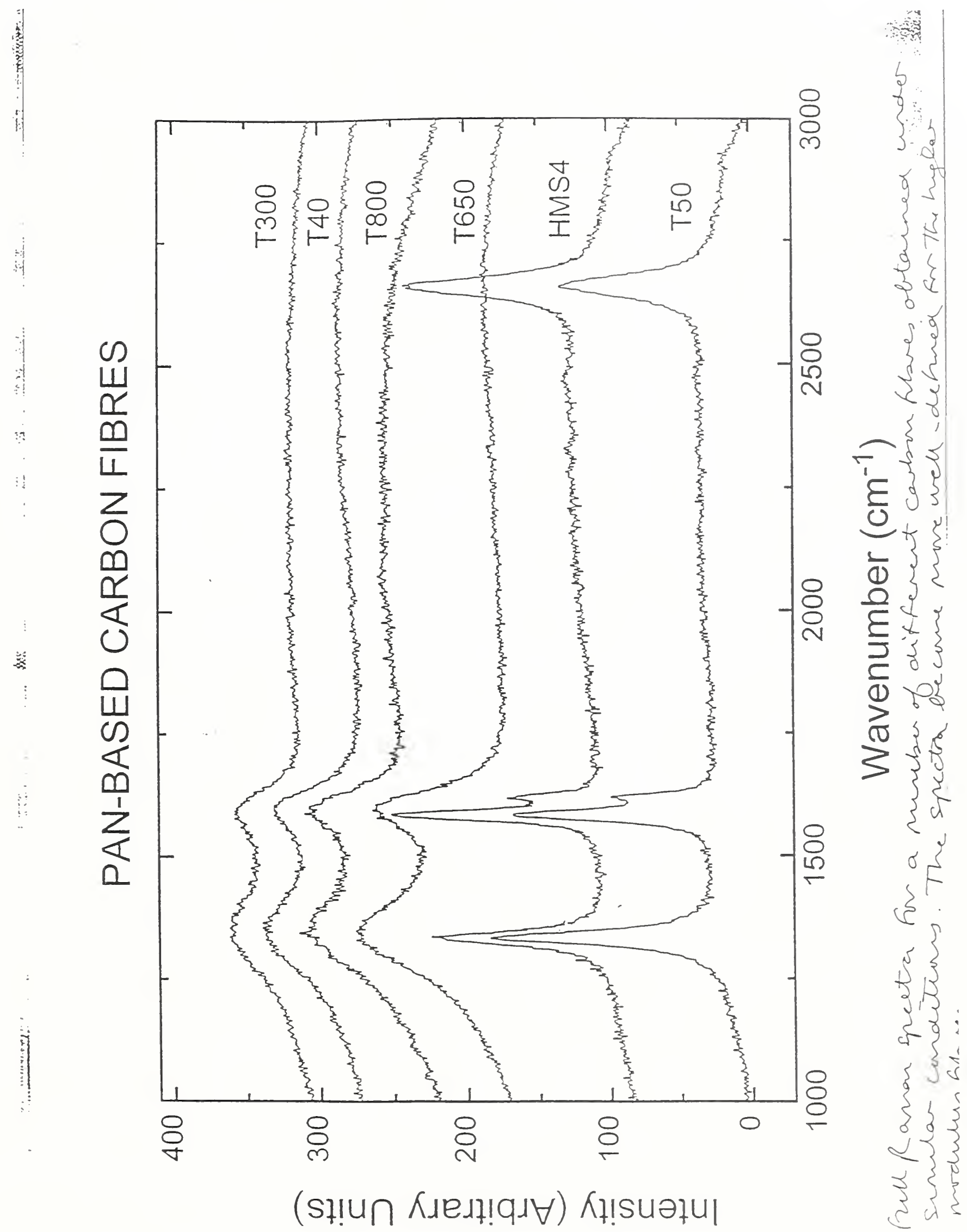

Slide 13. 


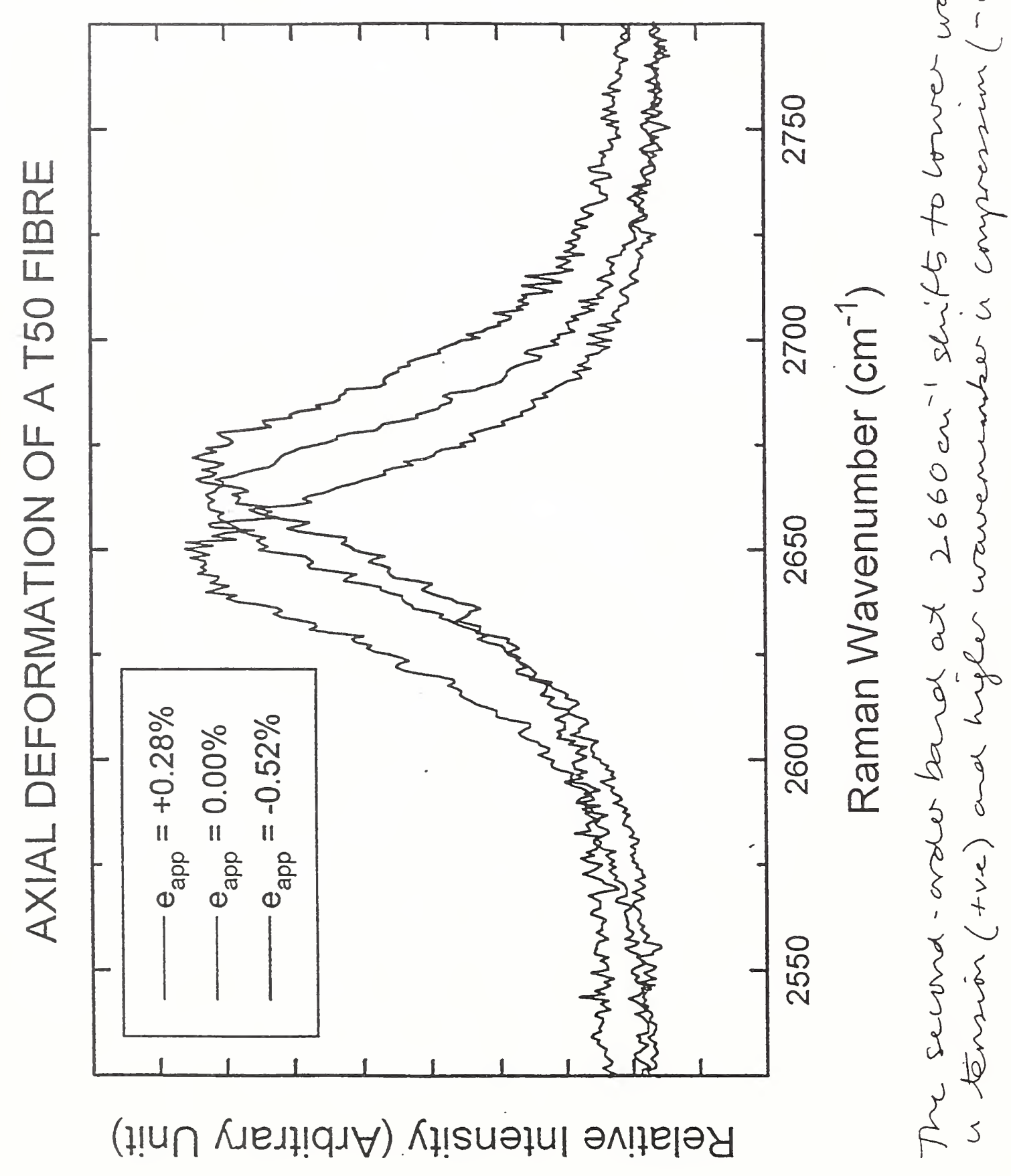

Slide 14. 


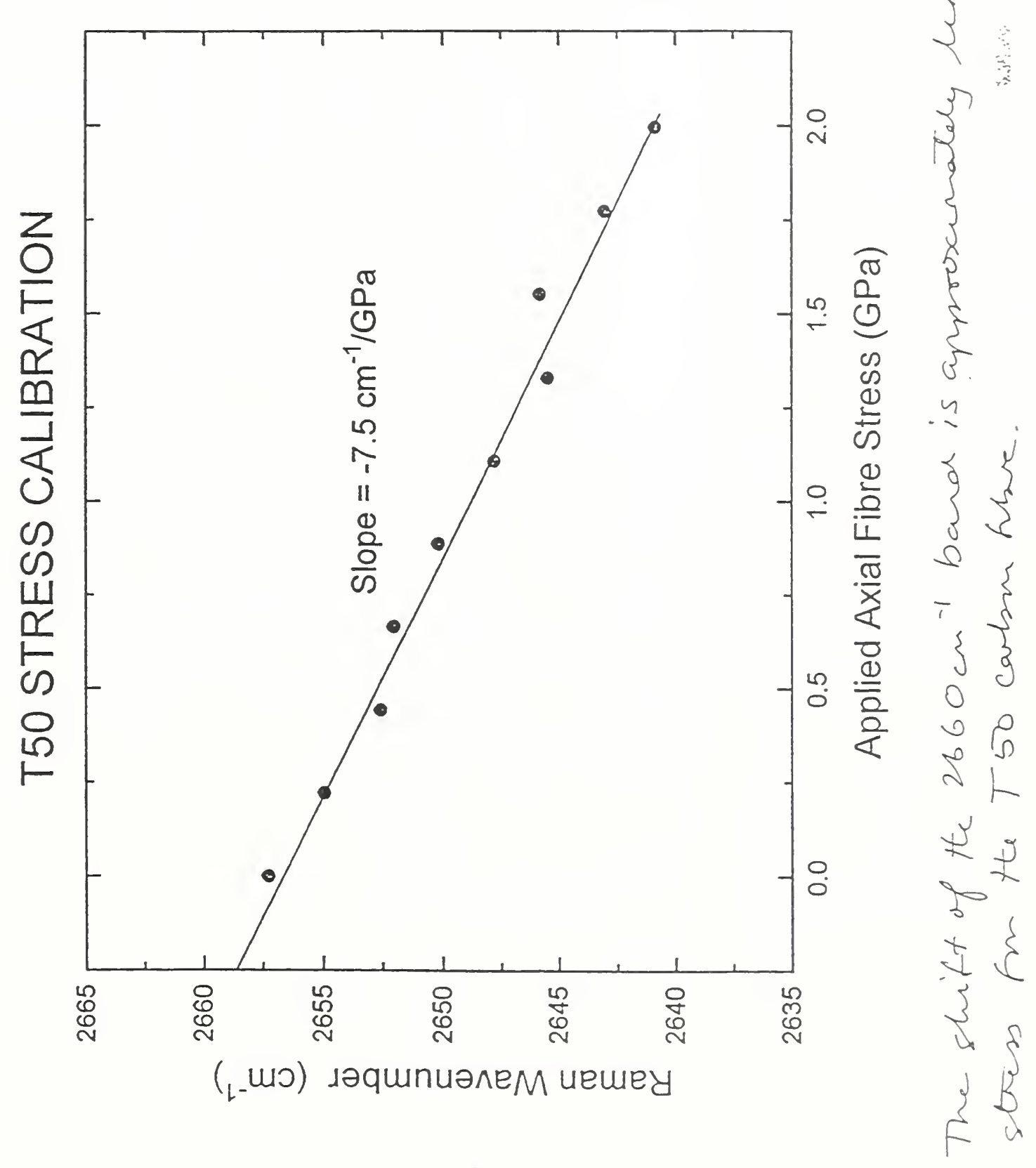

Slide 15. 


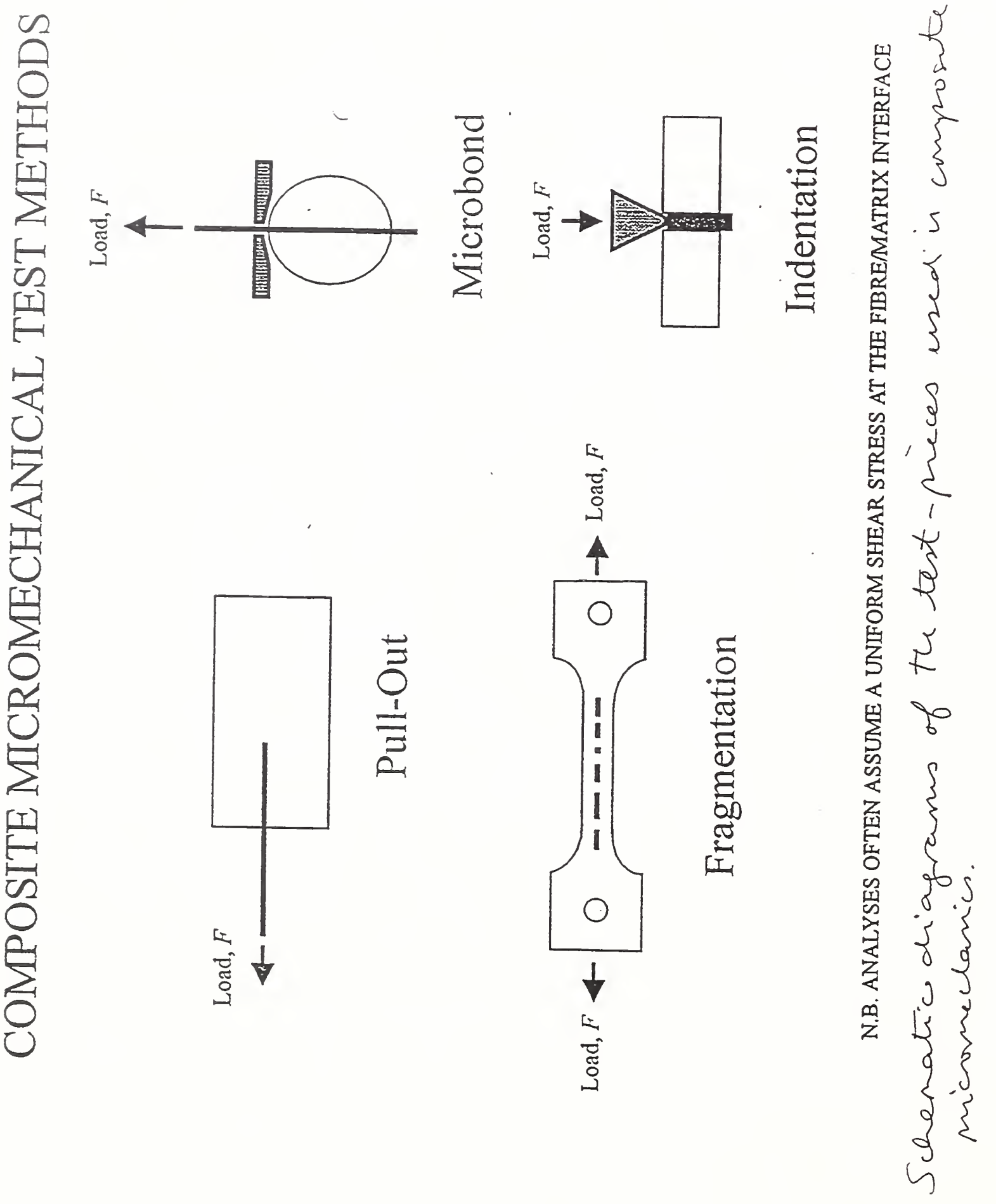

SIide 16. 

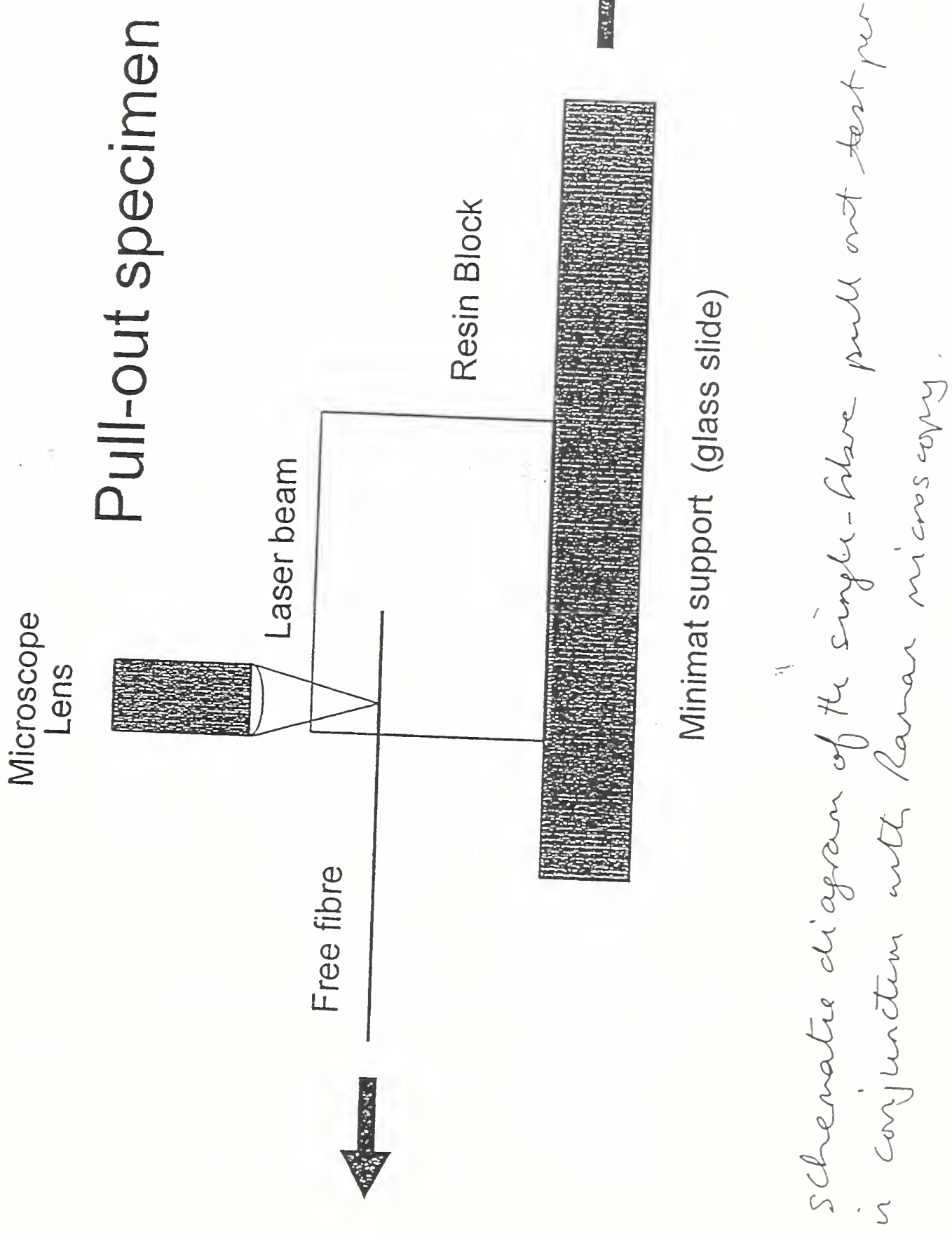

Slide 17. 


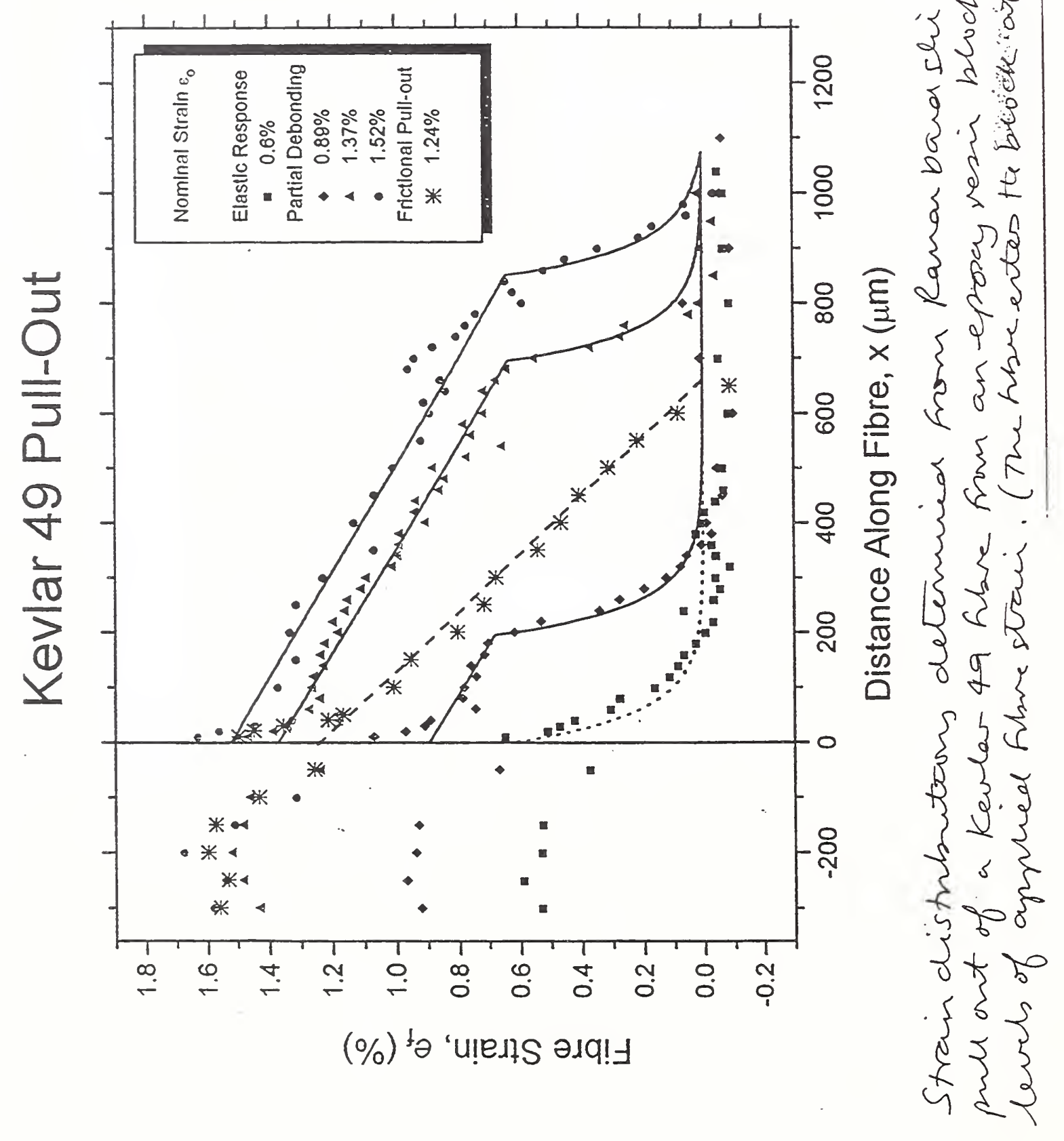

Slide 18. 


\section{BALANCE OF FORCES EQUILBRIUM}

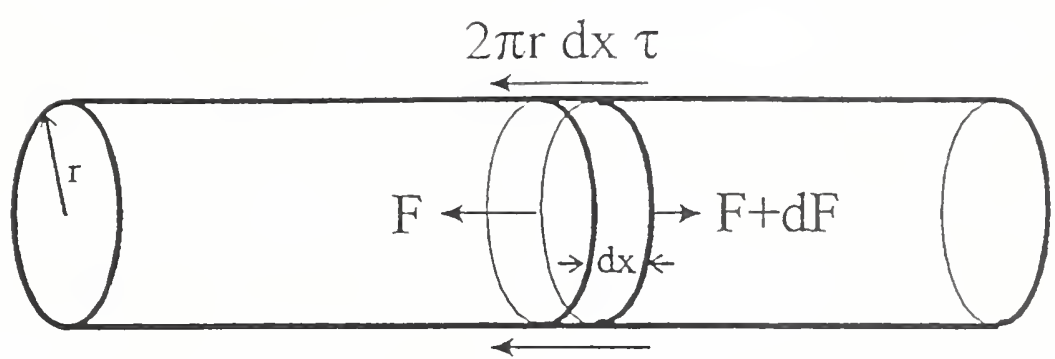

The interfacial shear stress along the fibre can be calculated by considering the equilibrium of the forces acting on an element of the fibre where the change in force is balanced by the shear stress at the interface.

$$
\mathrm{d} F=2 \pi r \tau d x
$$

Rearranging in terms of $\tau$ gives:

$$
\tau=\frac{1}{2 \pi r} \frac{\mathrm{d} F}{\mathrm{~d} x}
$$

The stress in the fibre can be calculated by dividing the force, $\mathrm{d} F$, by the cross sectional area of the fibre, $\pi r^{2}$, to give :

$$
\tau=\frac{r}{2} \frac{d \sigma_{f}}{d x}
$$

This can easily be converted to calculate the interfacial shear stress with respect to the fibre strain.

$$
\tau=\frac{r}{2} E_{f}\left[\frac{\mathrm{d} e_{f}}{\mathrm{~d} x}\right]
$$

where $\mathrm{d} e / \mathrm{d} x$ is the slope of the strain distribution curve at any point along the fibre. 


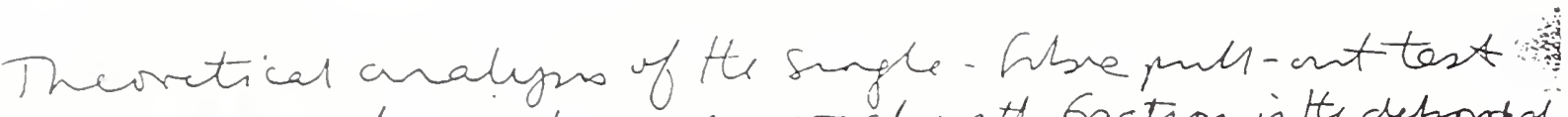

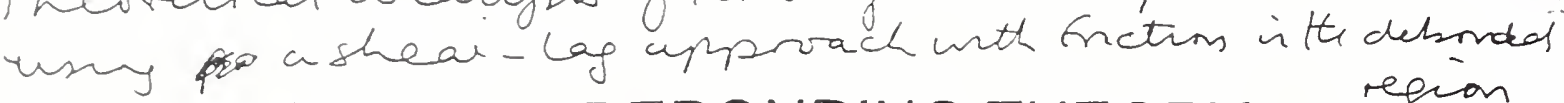

\section{PARTIAL DEBONDING THEORY} repan.
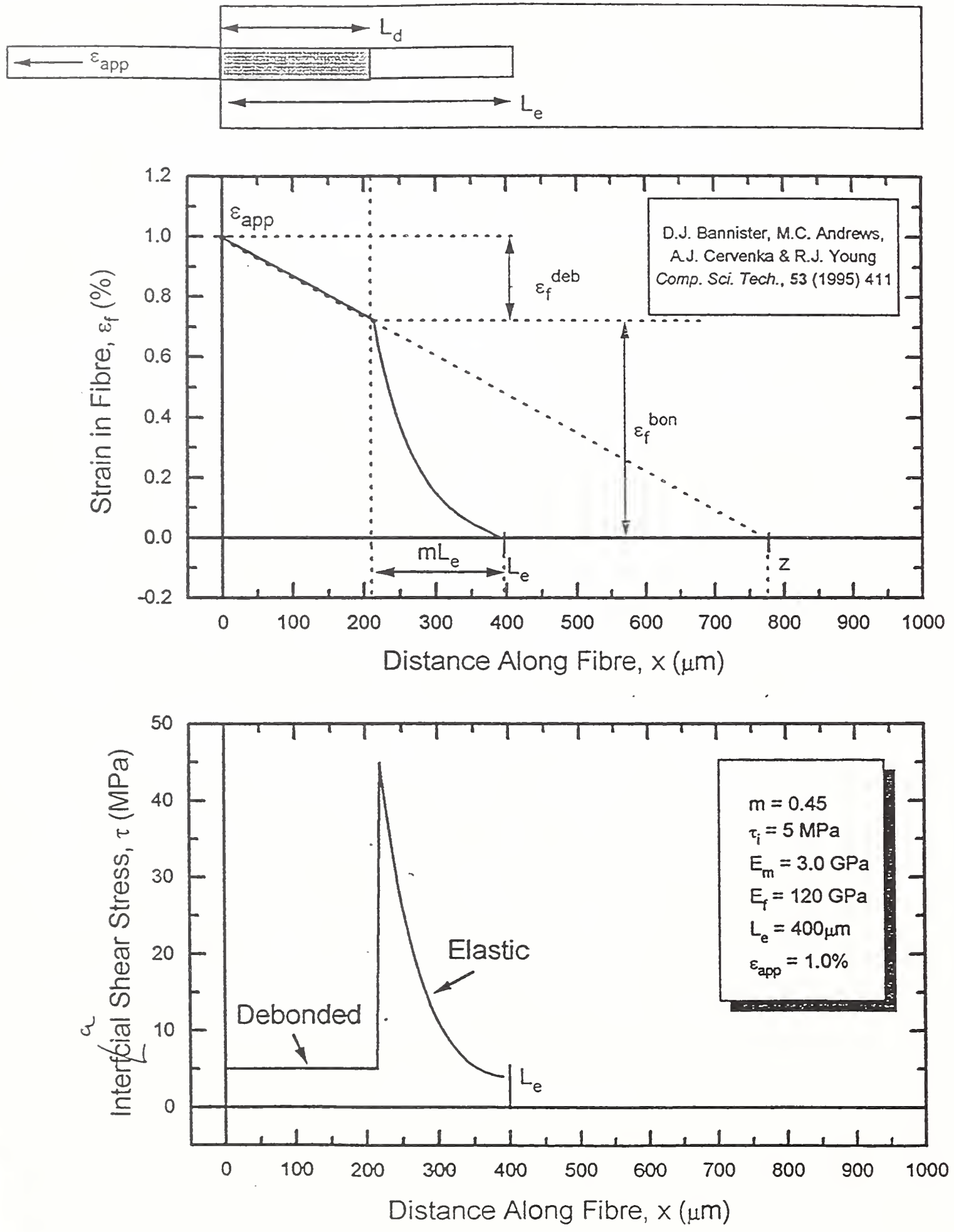

Slide 20. 

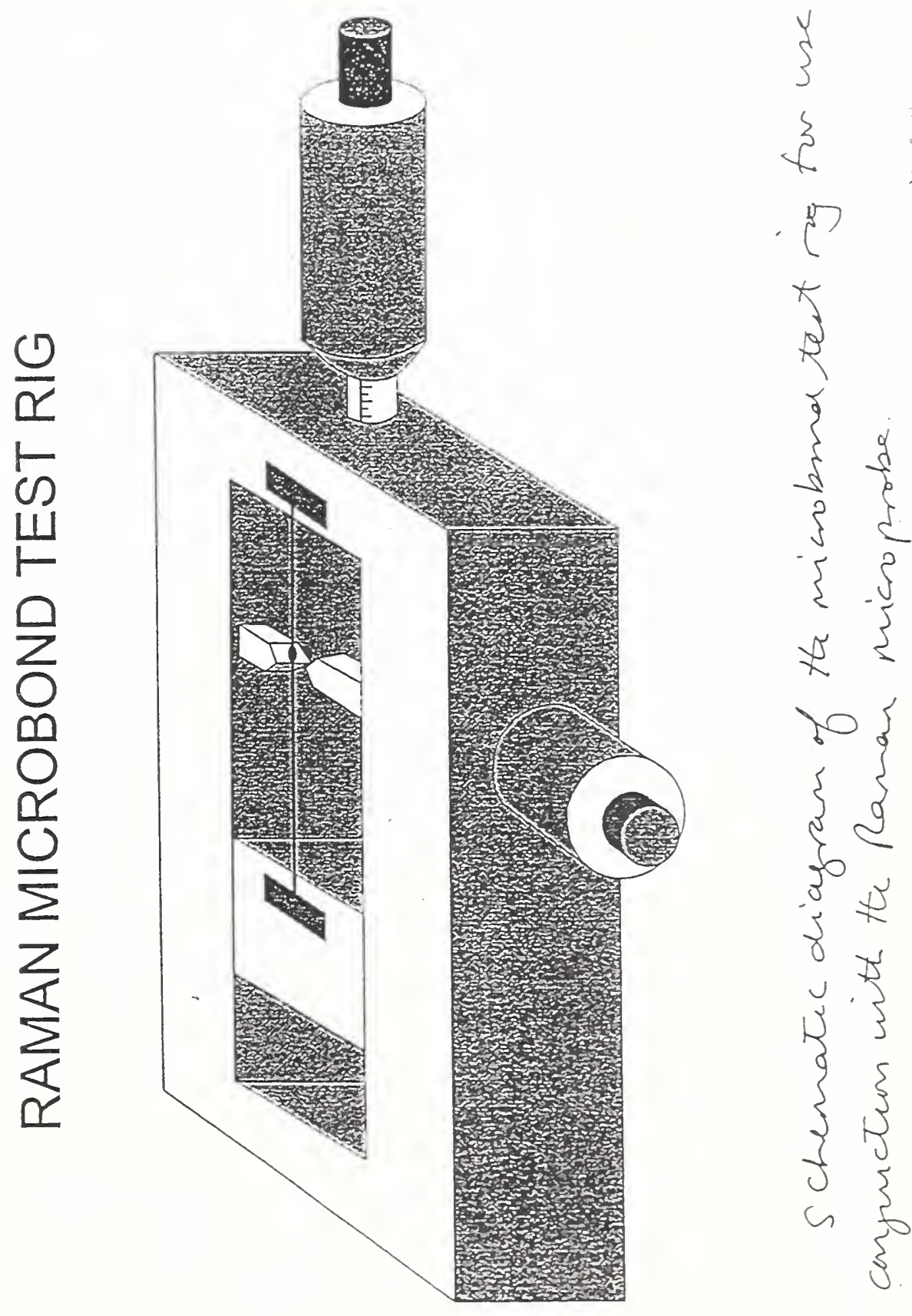

$8 \pi$

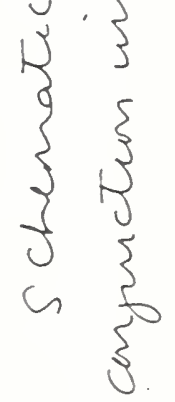

Slide 21. 


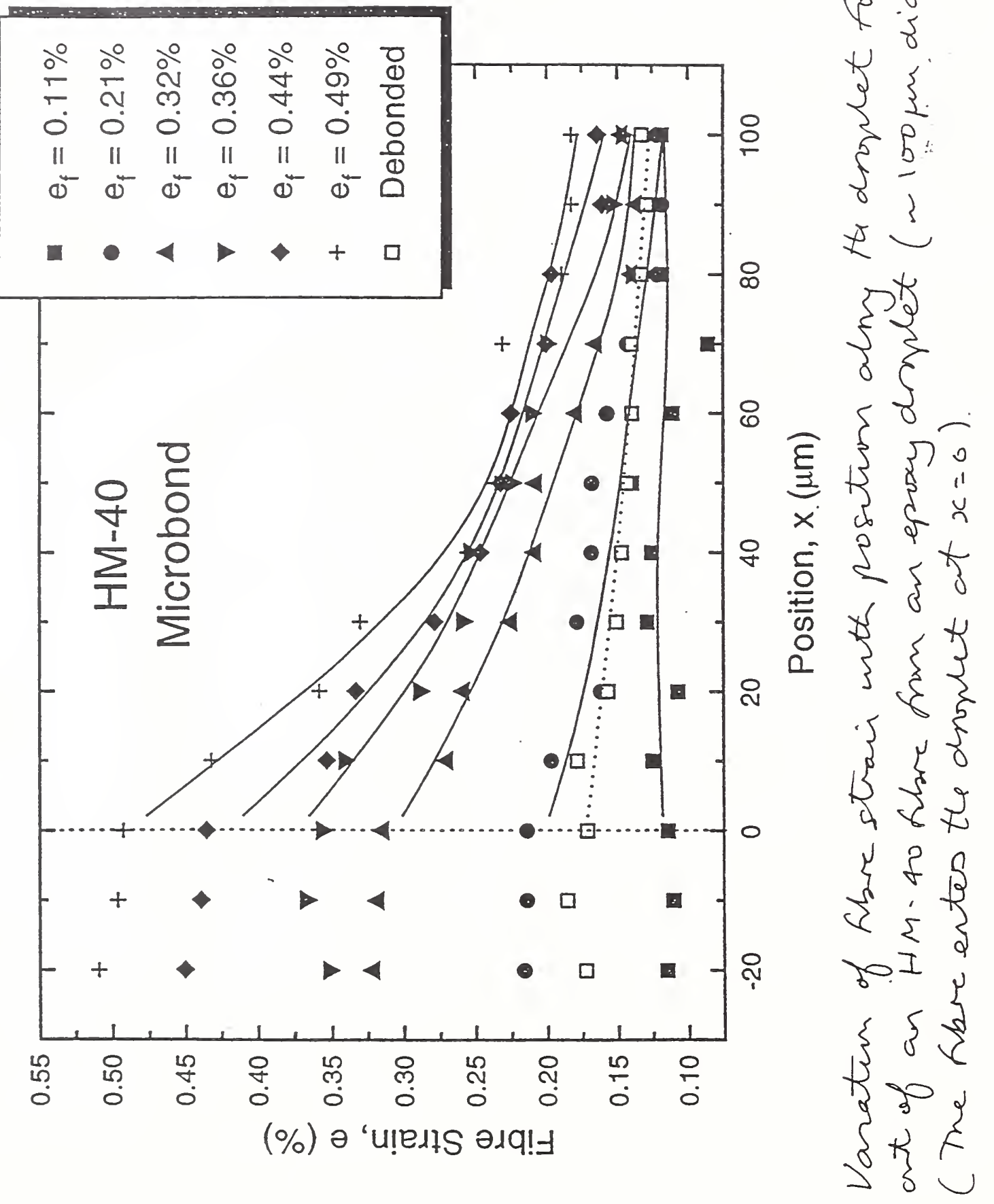

Slide 22. 


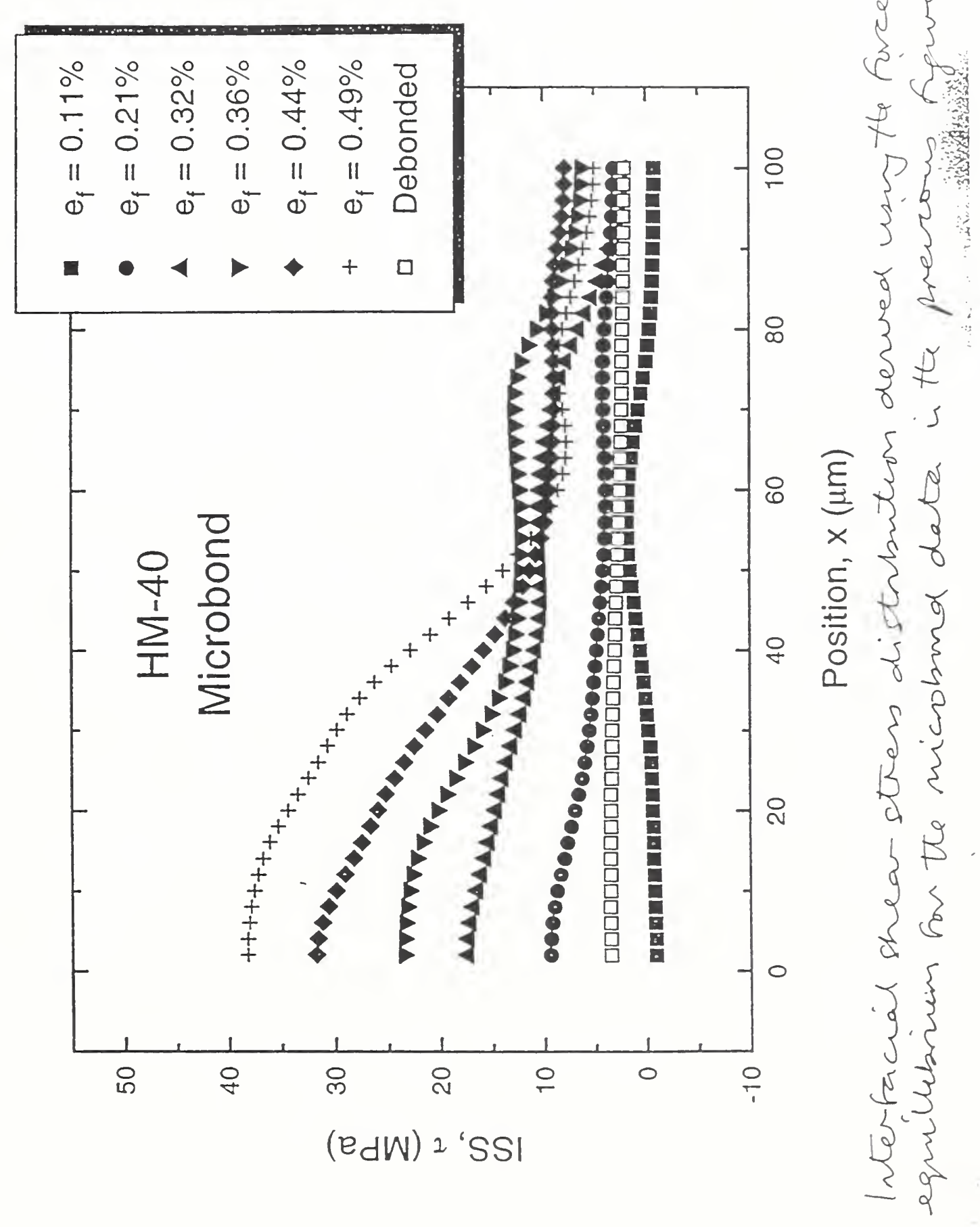

Slide 23. 


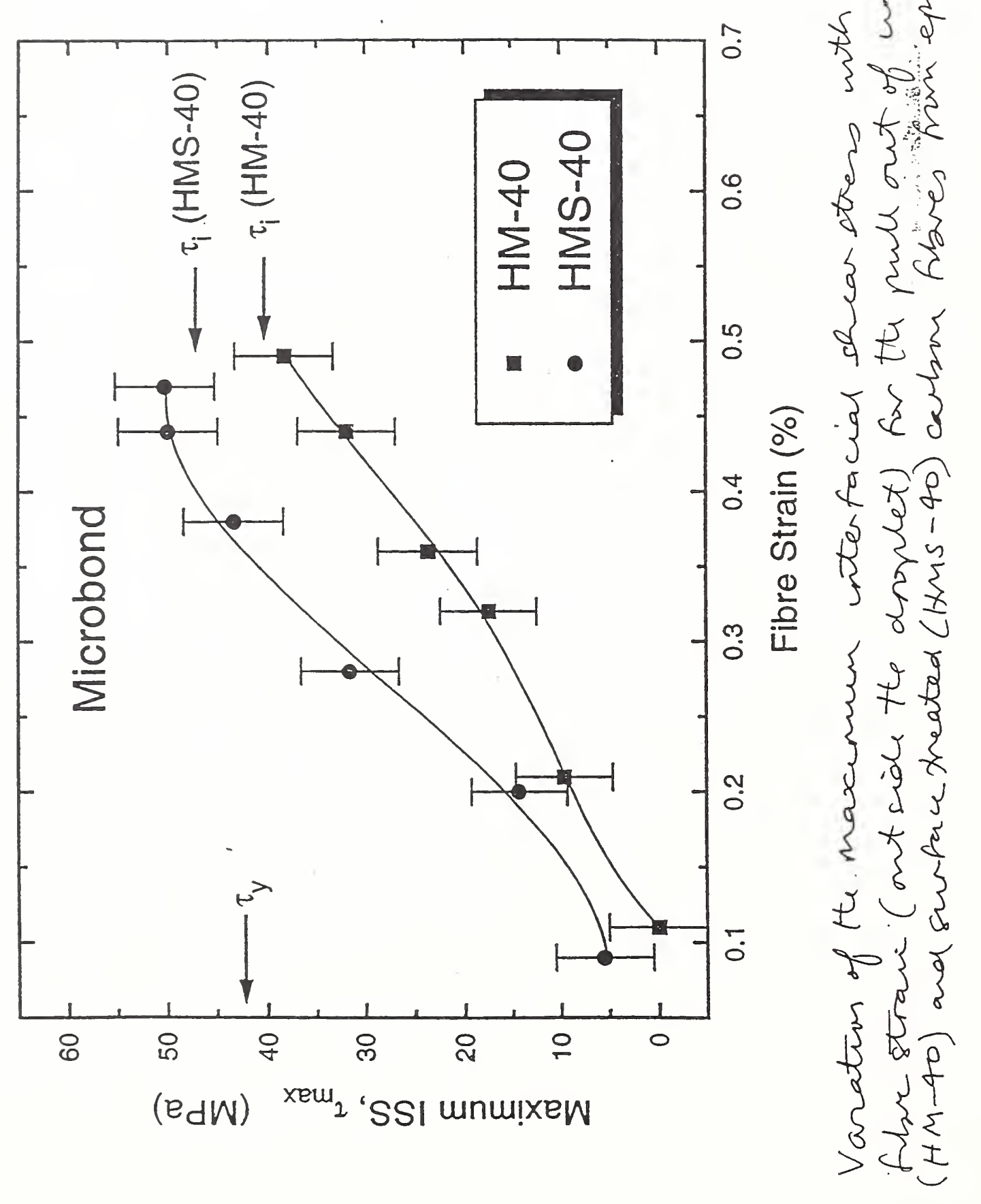

Slide 24. 

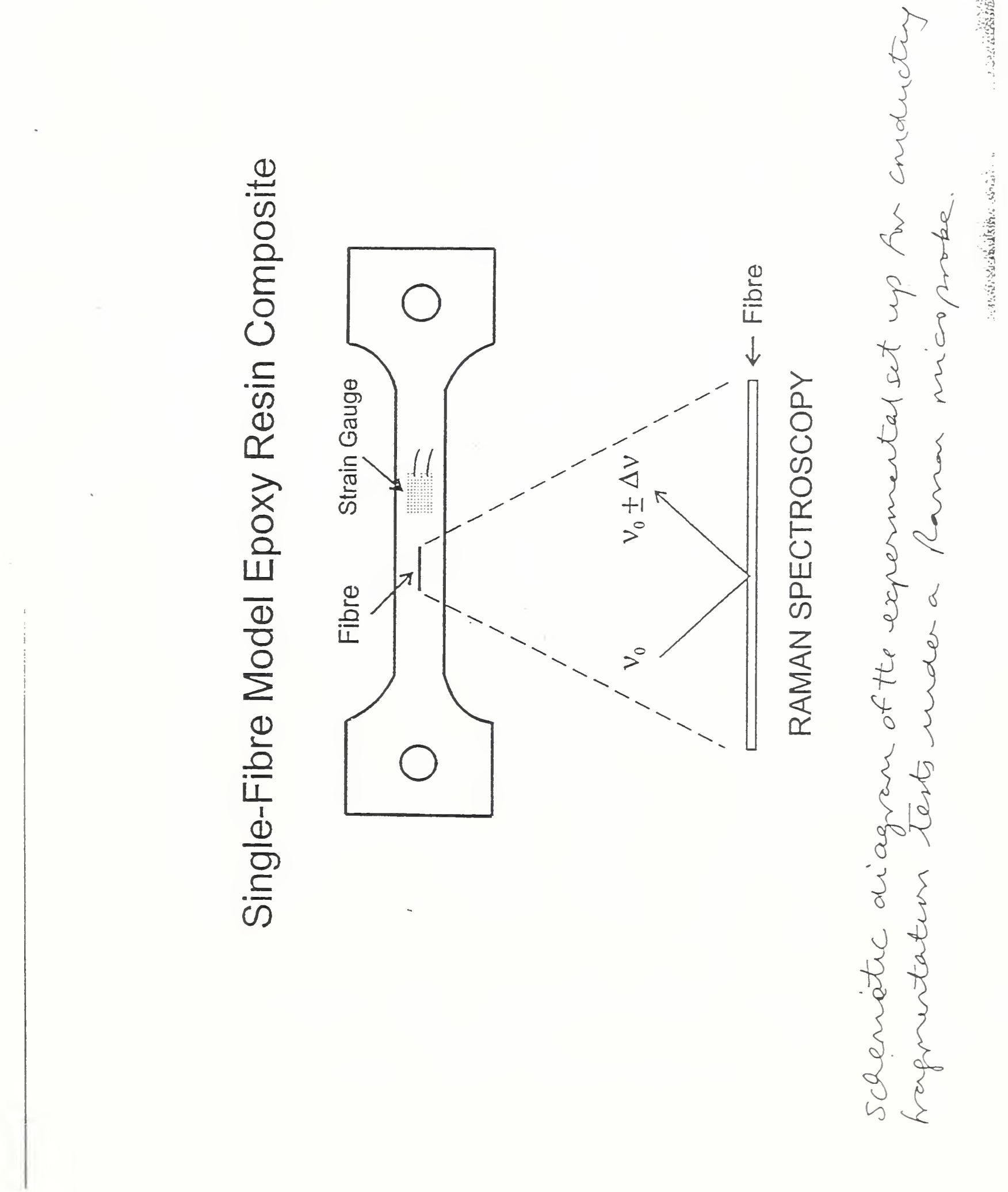

S1ide 25. 


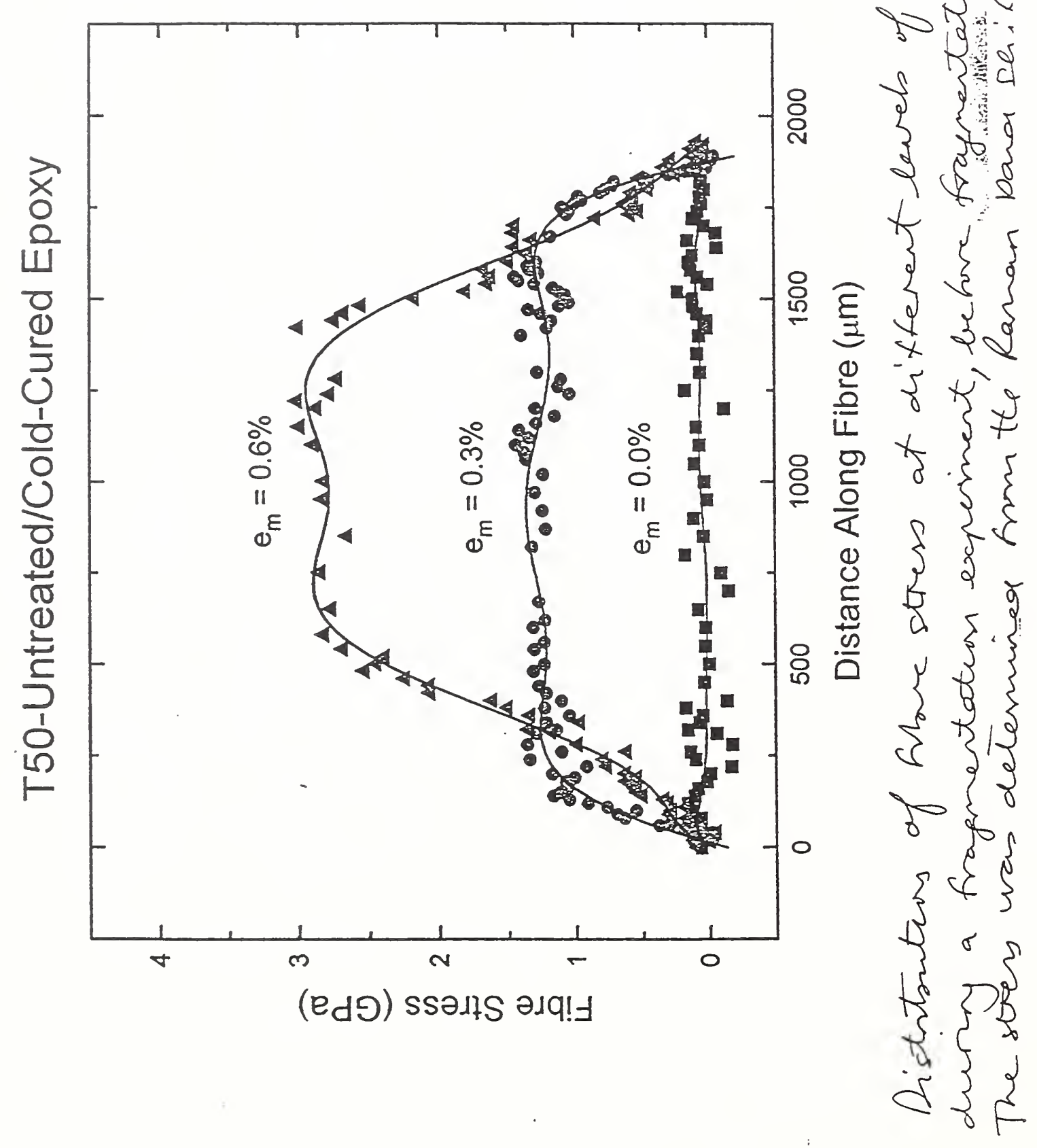

Slide 26. 


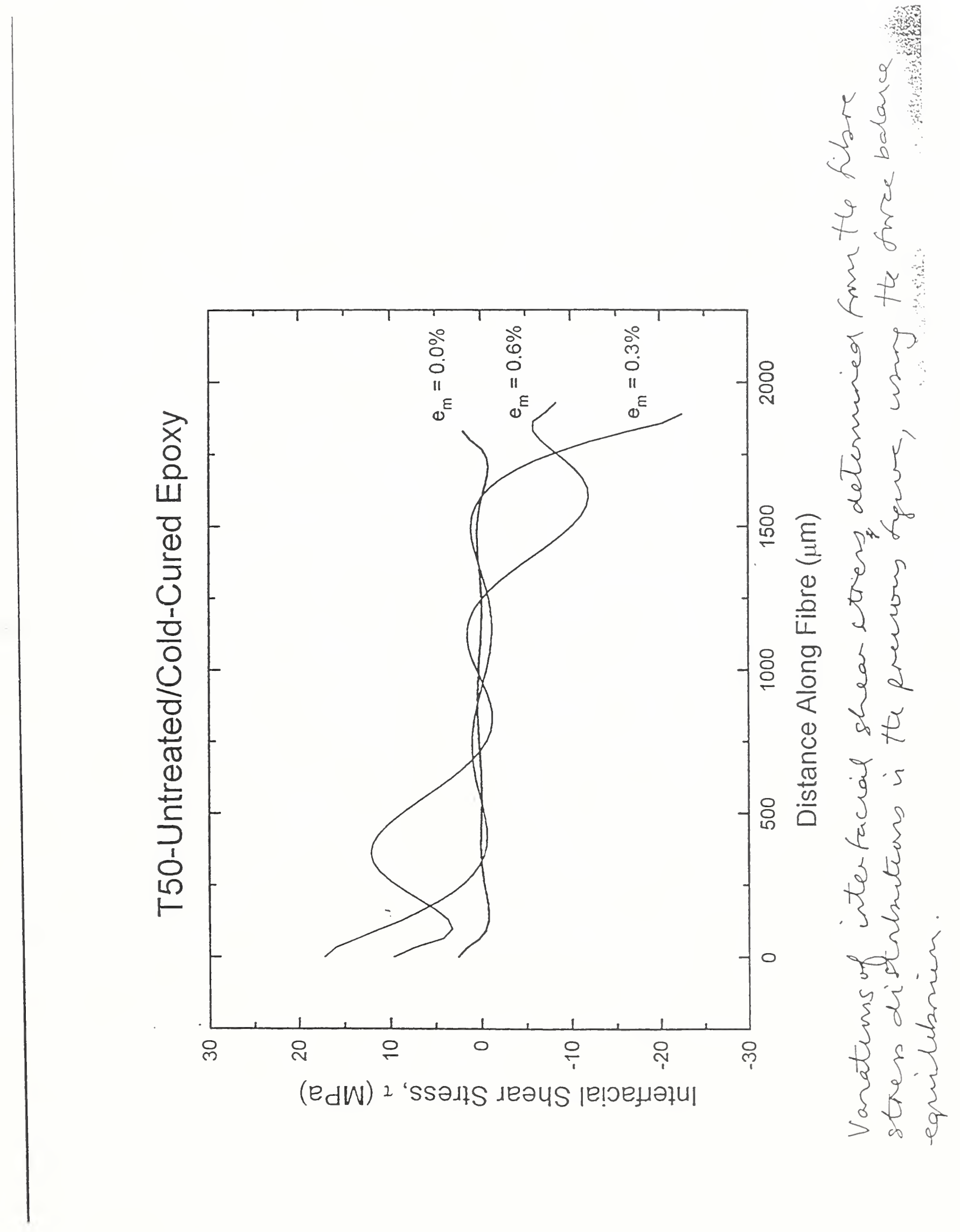

Slide 27. 


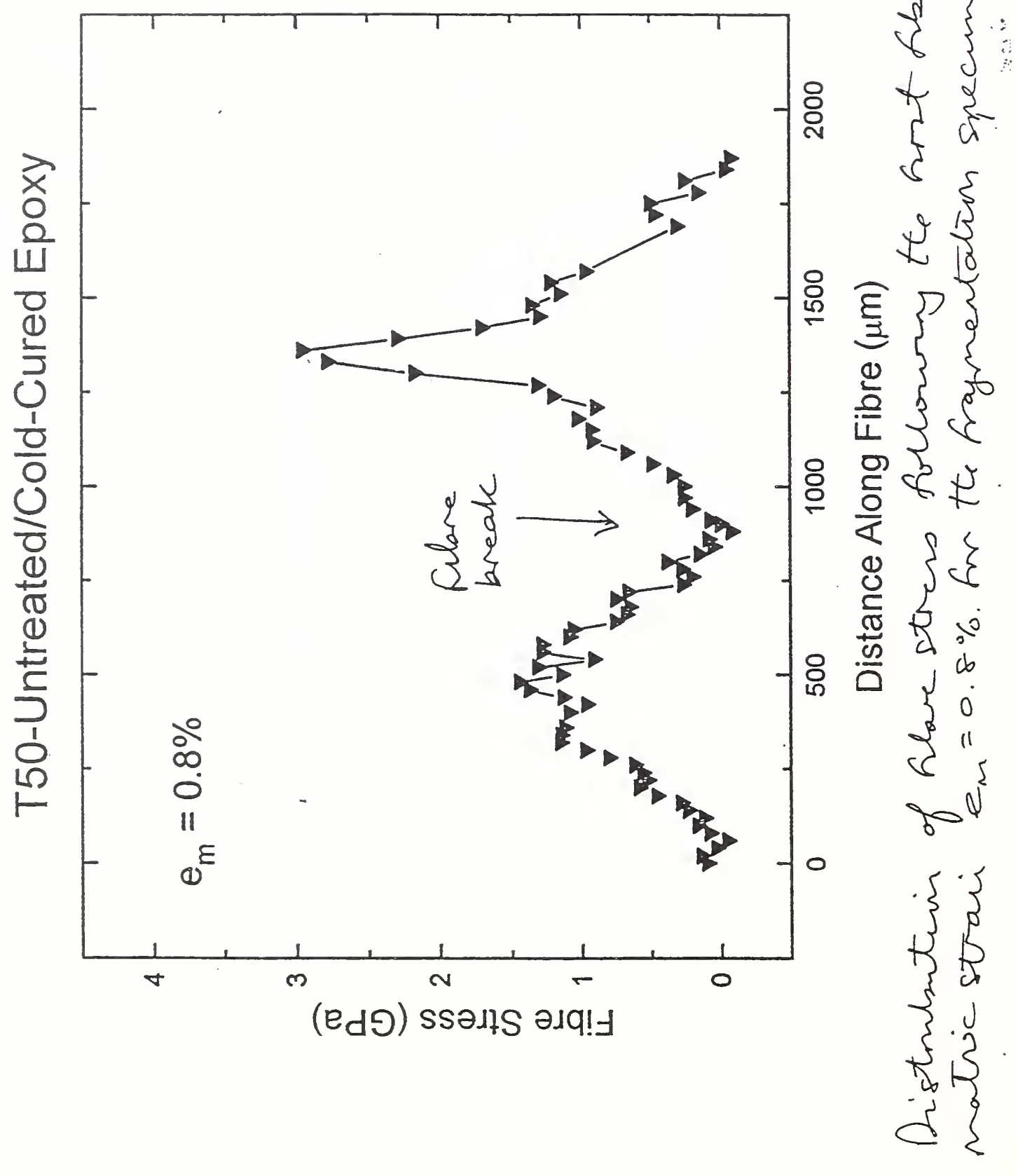

Slide 28. 


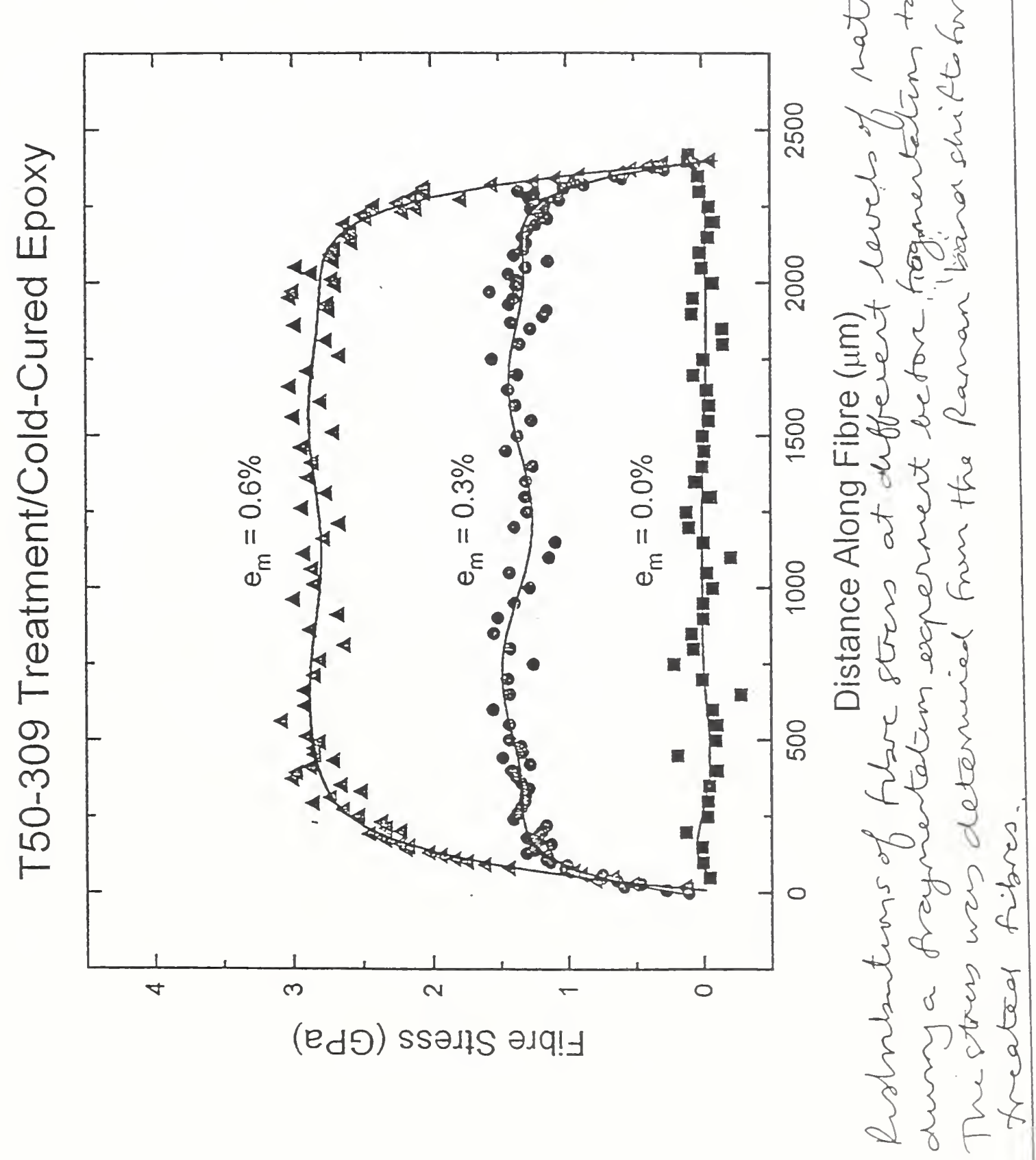

SIide 29. 


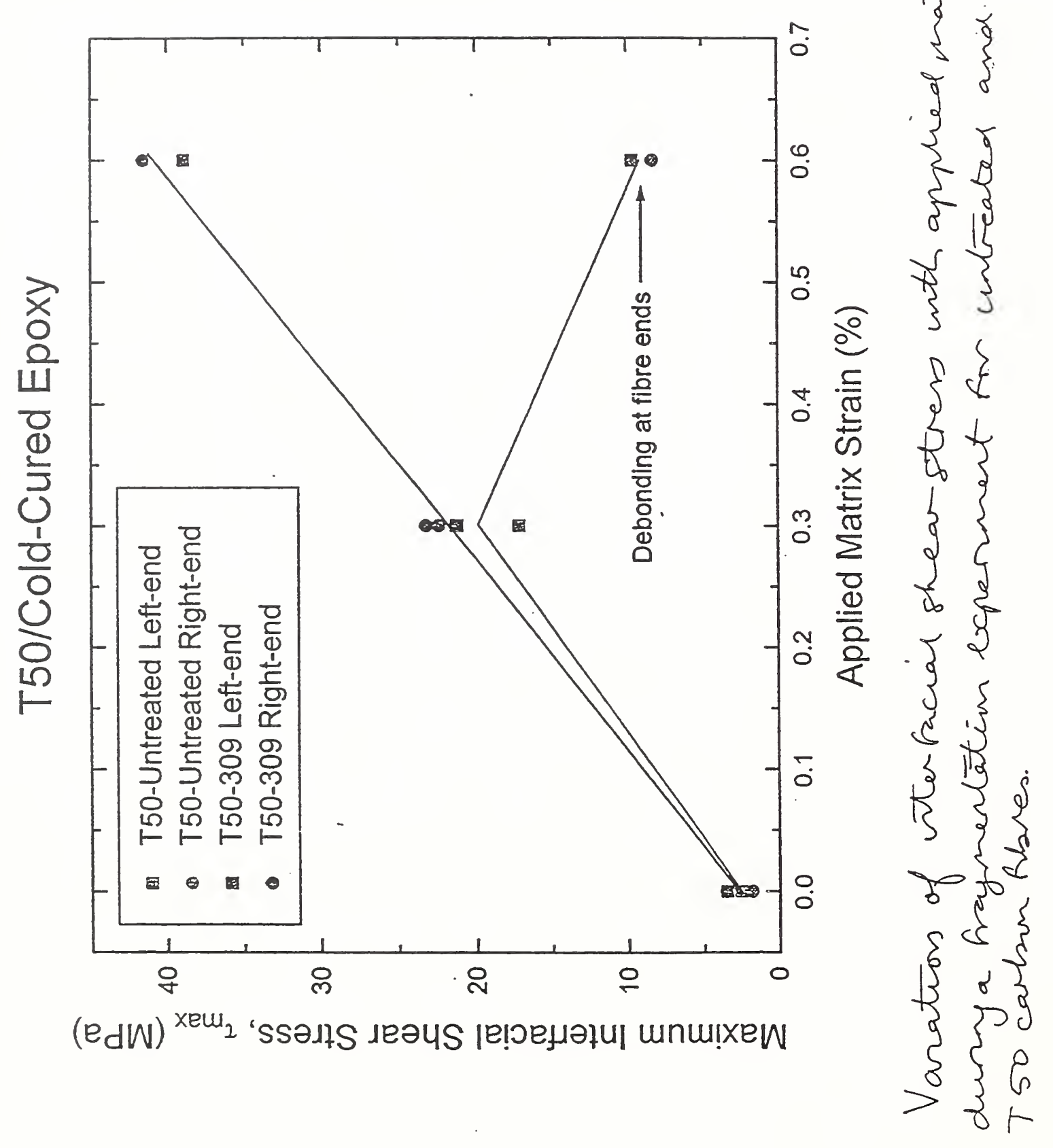

Slide 30. 


\section{STRAIN DEPENDENCE OF RAMAN BANDS TENAX HMS-40 CARBON FIBRES}
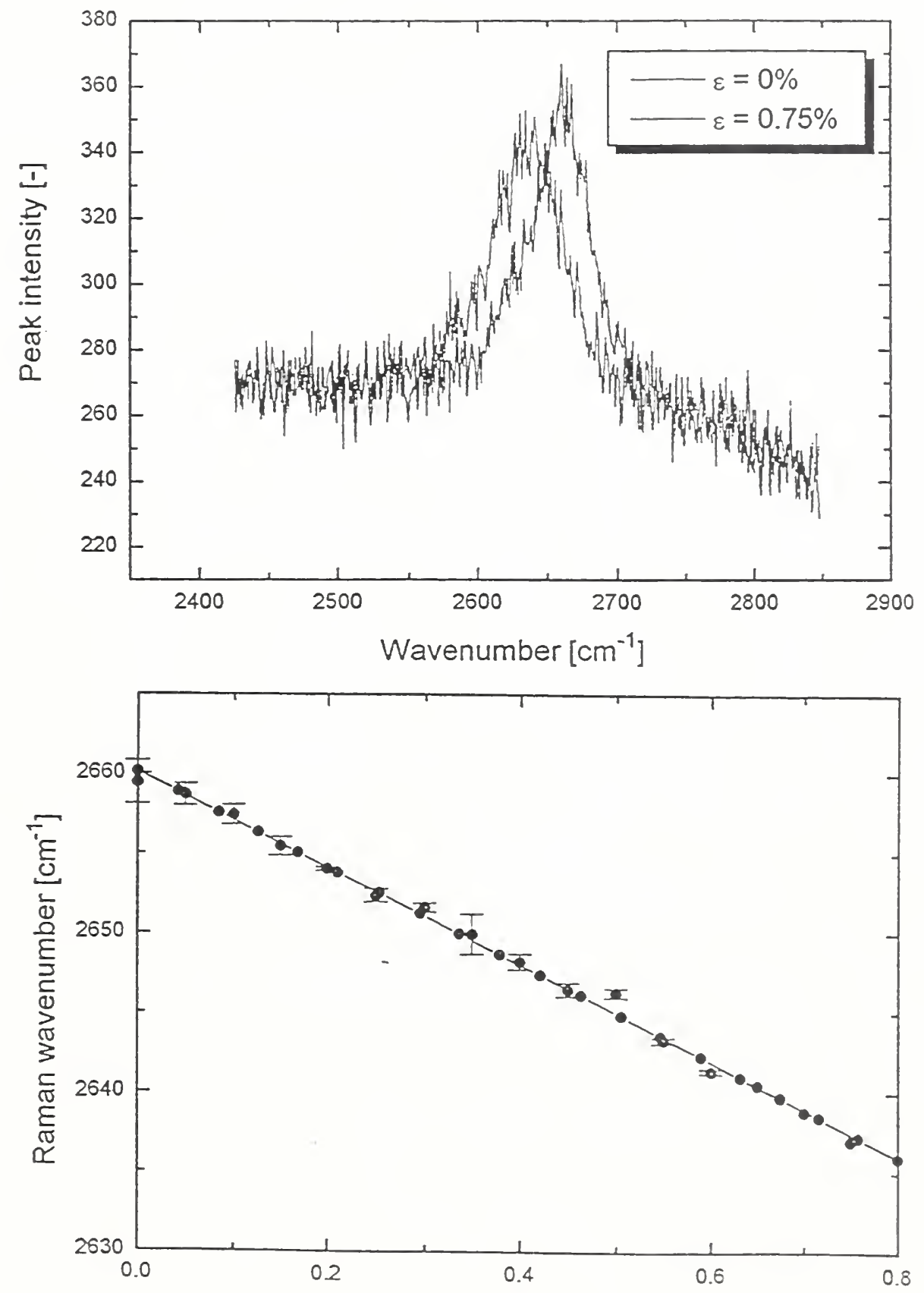

Fibre strain [\%]

The $2660 \mathrm{~cm}^{-1}$ band shifts to coverwaverumber in torsin 


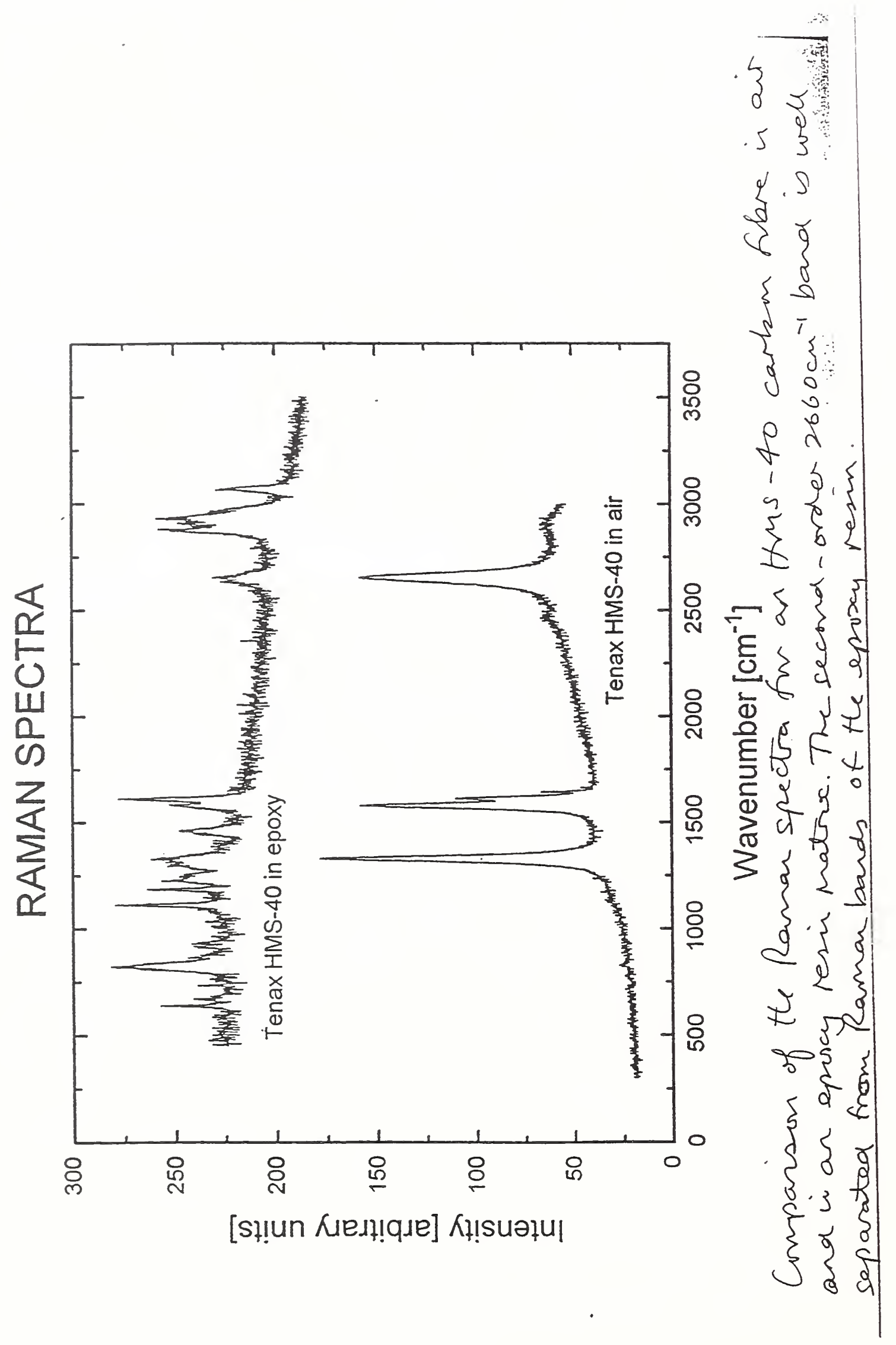

Slide 32. 


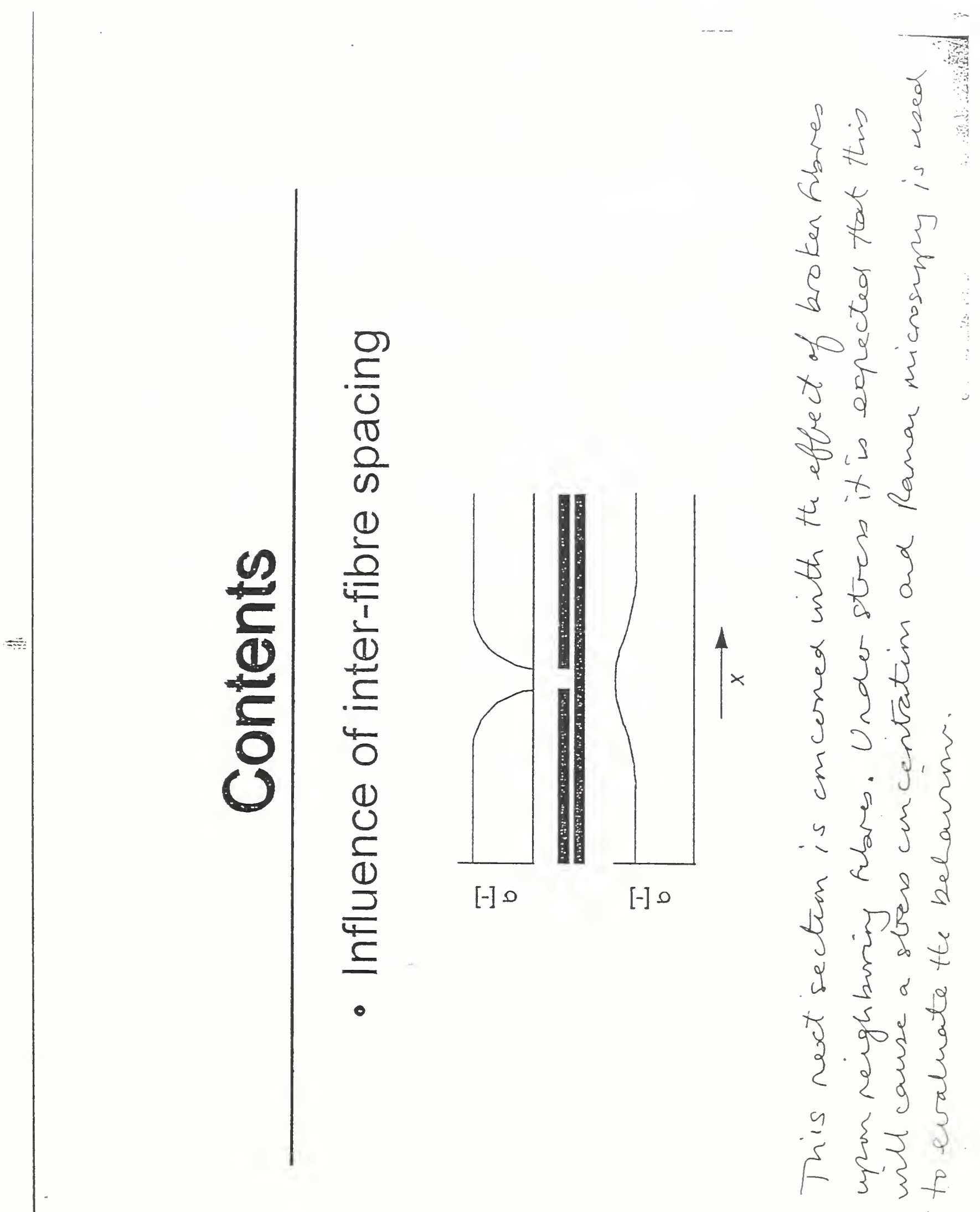

Slide 33. 


\section{HMS-40/EPOXY MICROCOMPOSITES \\ CLOSELY-SPACED FIBRES $(\varepsilon=0.9 \%)$}

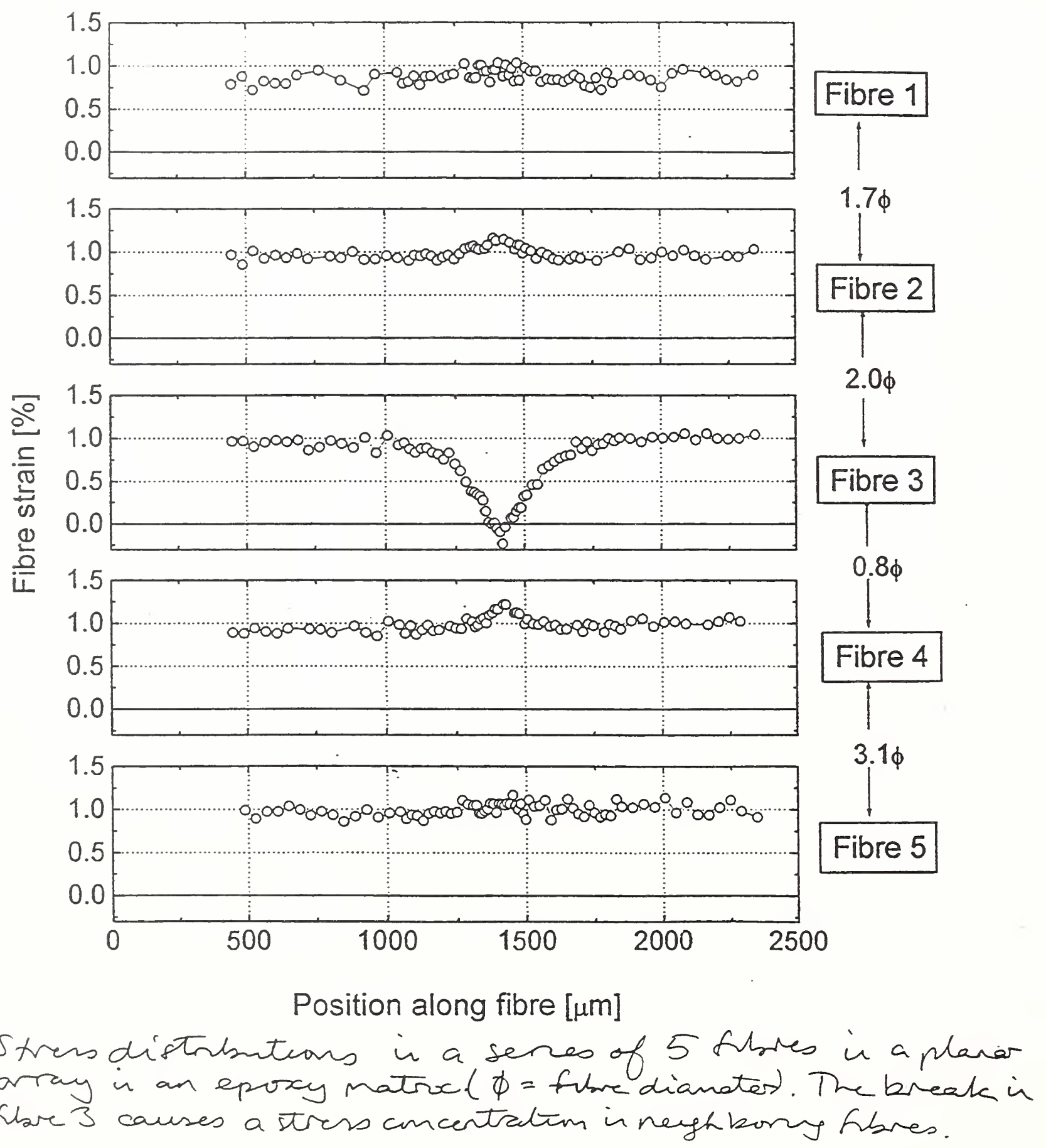

Slide 34. 


\section{HMS-40/EPOXY MICROCOMPOSITES \\ CLOSELY-SPACED FIBRES $(\varepsilon=1.1 \%)$}

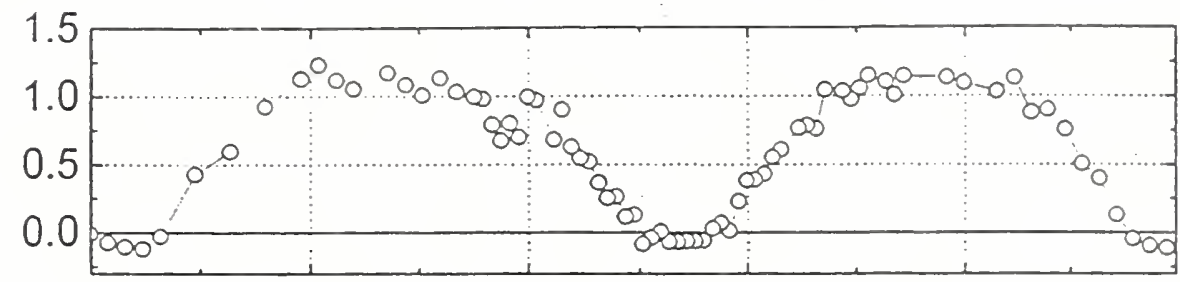

\section{Fibre 1}
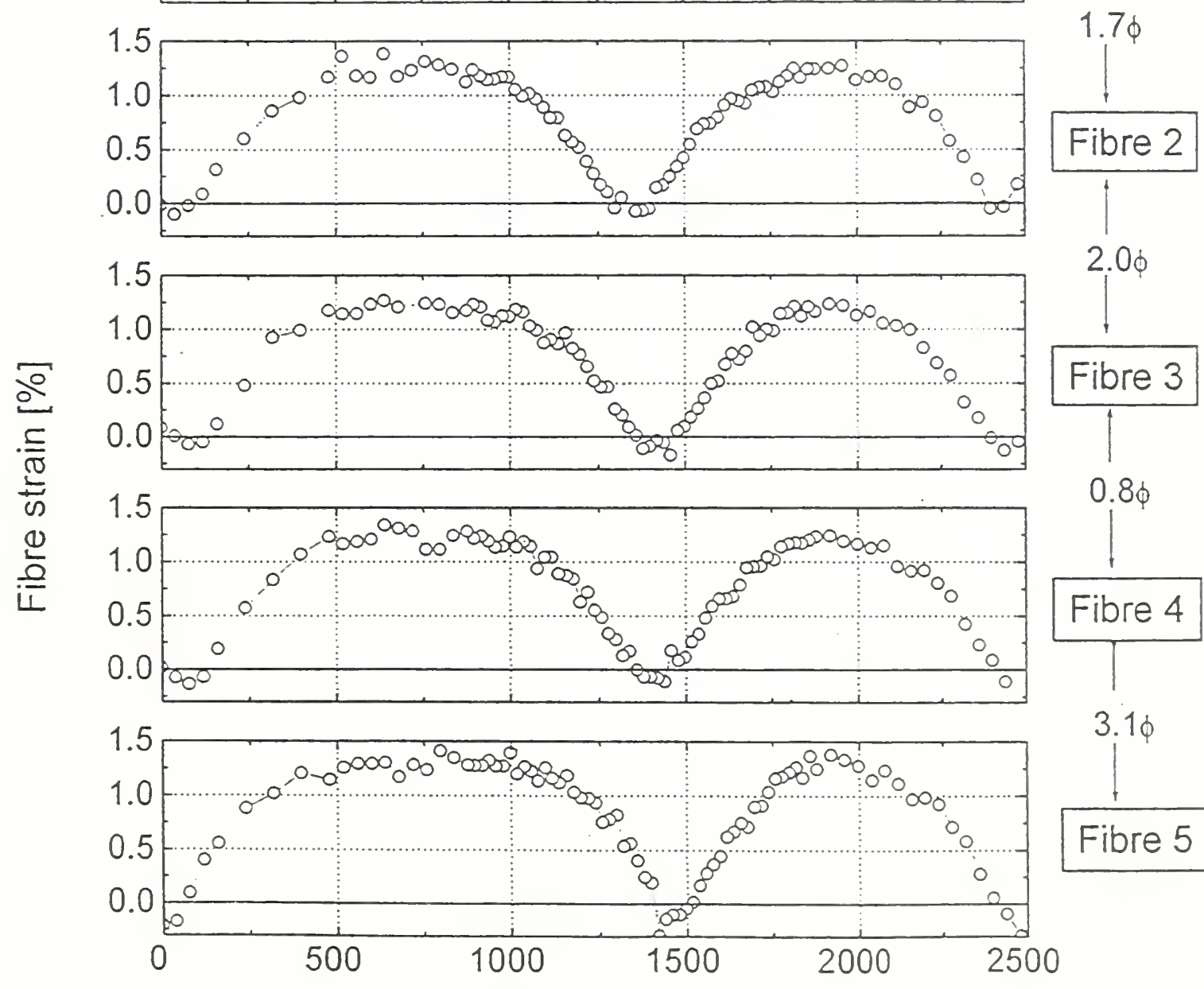

Samequecurian Position along fibre $[\mu \mathrm{m}]$

Ats in the prenions fave but wo the matre strail increased to 1.1\%. All five fibres have fragmented and the breaks line up for these closely-spaced firres. 


\section{HMS-40/EPOXY MICROCOMPOSITES STRESS CONCENTRATIONS}

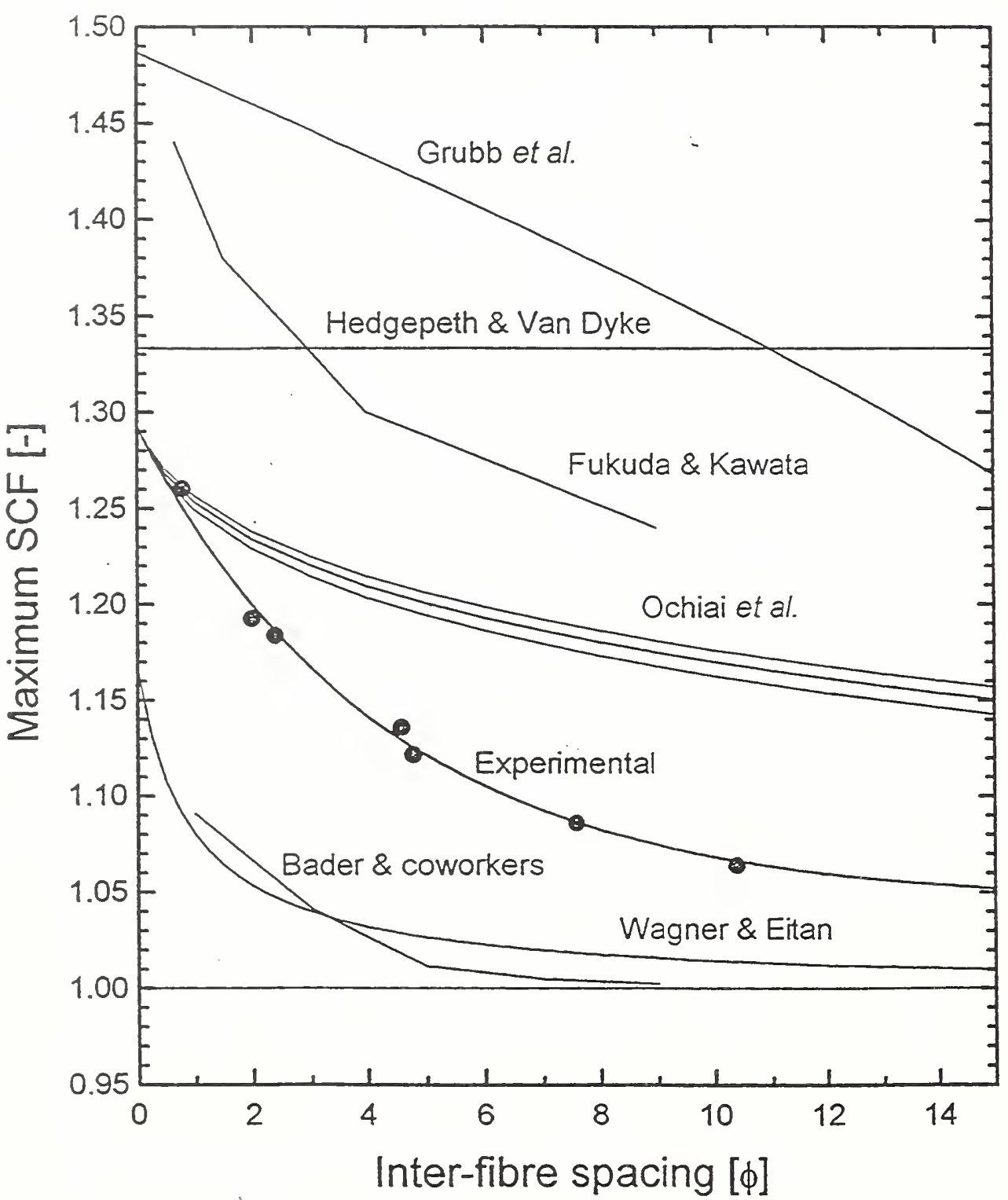

Deperdence of stress concertatum factor (scf) upm inter-Hore separatems For adyacent HMI-40 carbon fibres. The expermentas data ar from the Ranan bard shifts and the otter curves were derived therretically (urrkes ndicated) 


\section{Pre-cracked Compact Tension Specimen}

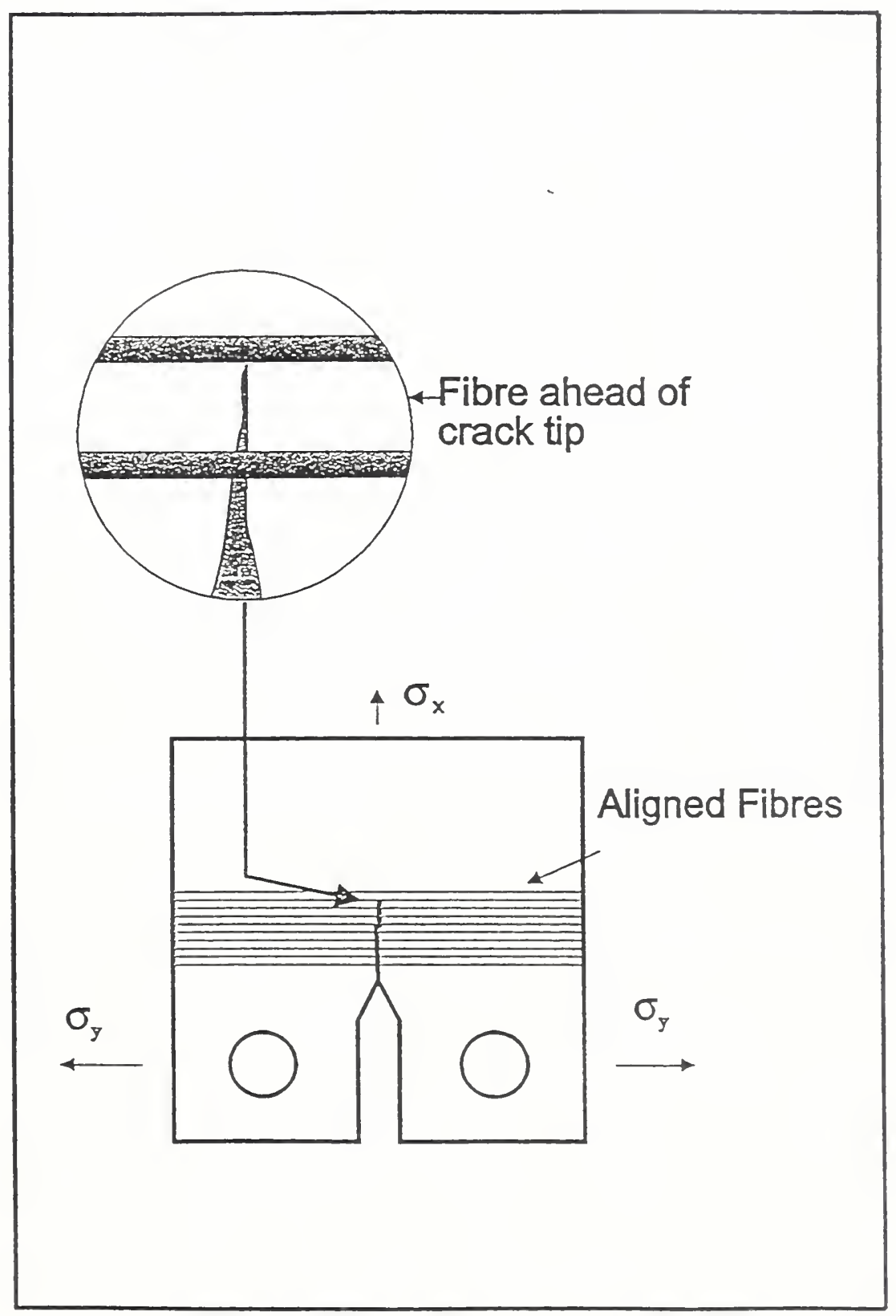

Specimer used to determice sters distrbutions in arrays of fubres bridging a crack in an epoxy cmpact tersios specimen. 


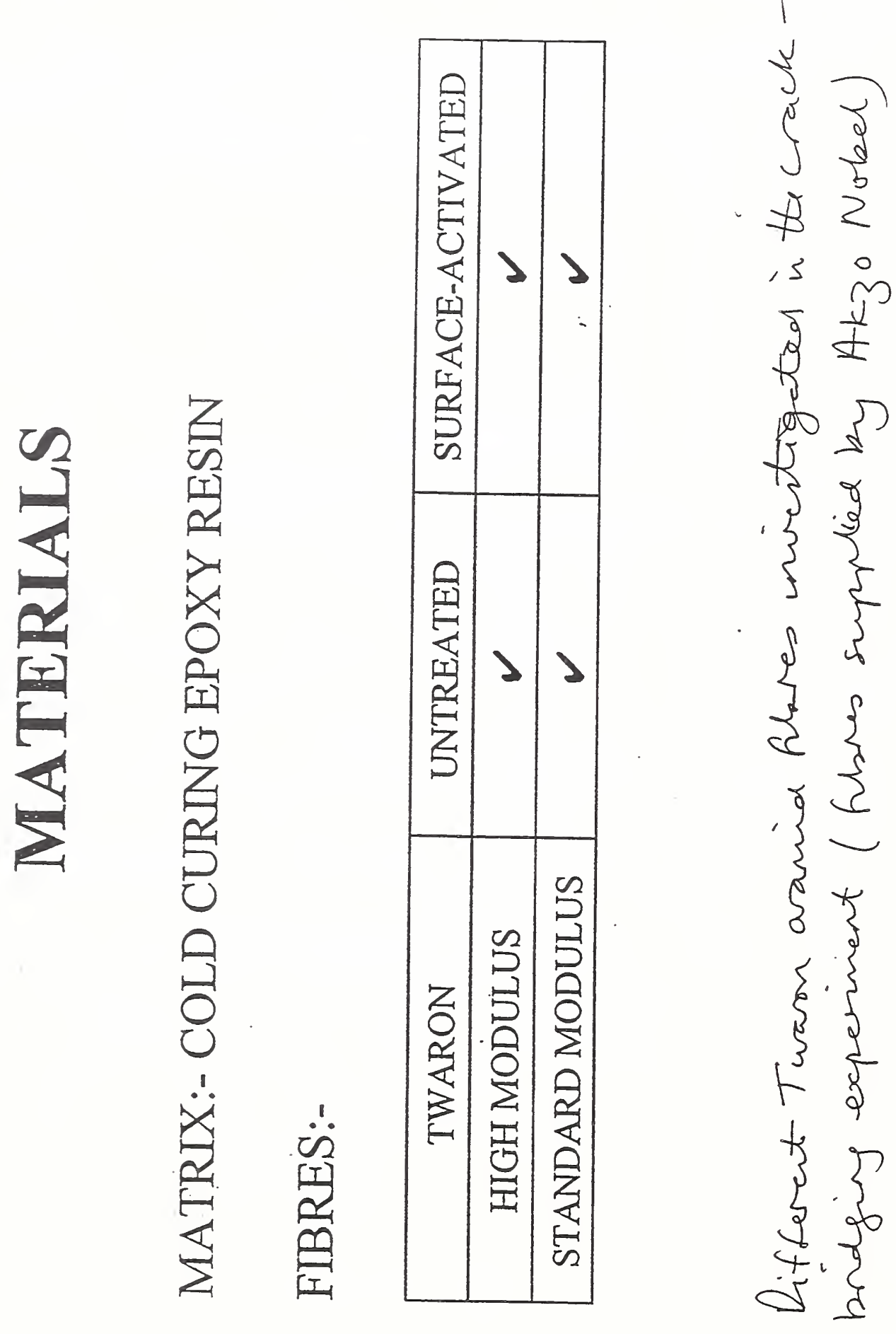

Slide 38. 
Expermentel and therveticas stain distrhations forming

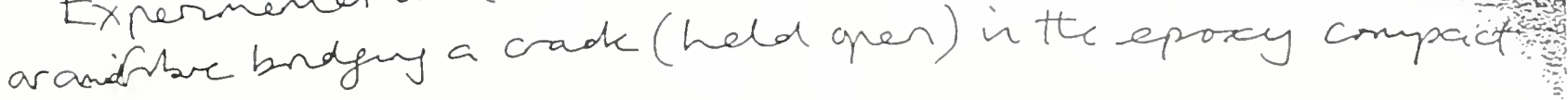

tension specumen.

\section{Pre-Cracked Specimen}

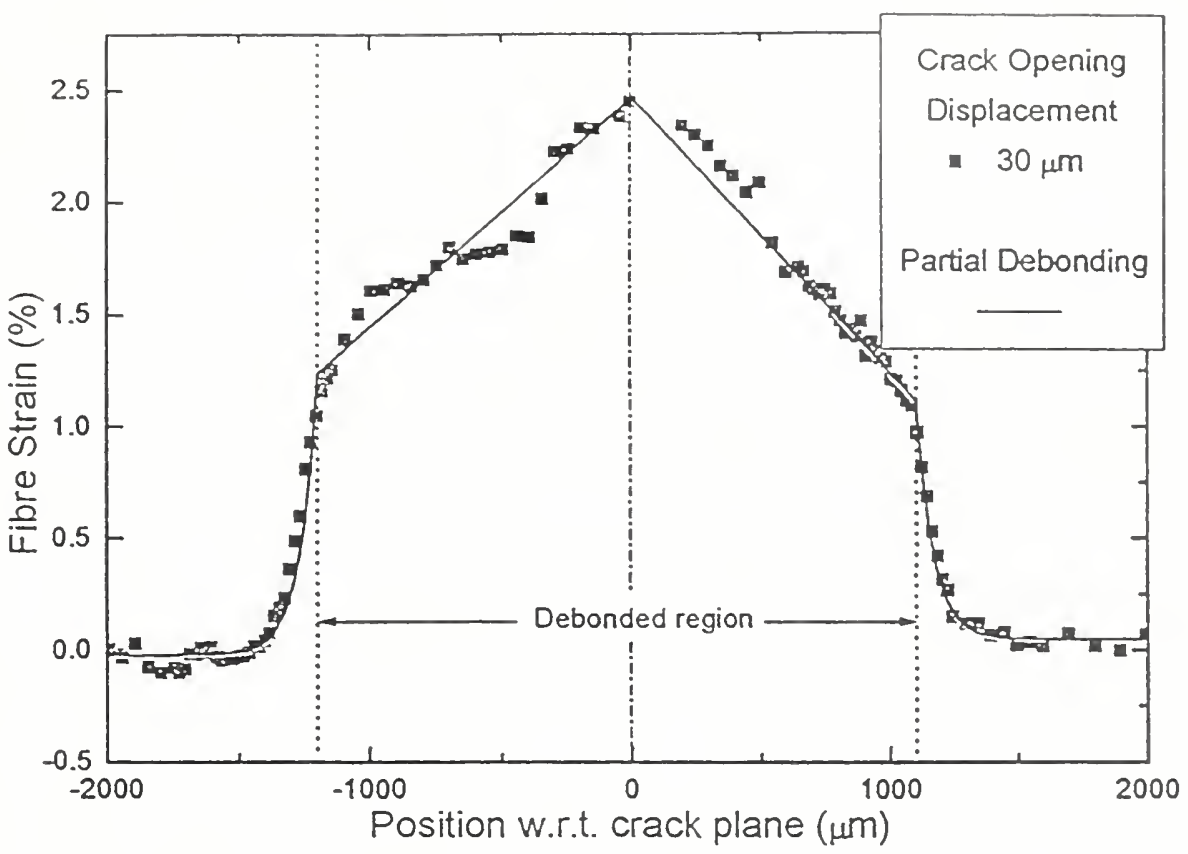

Partial Debonding Model*

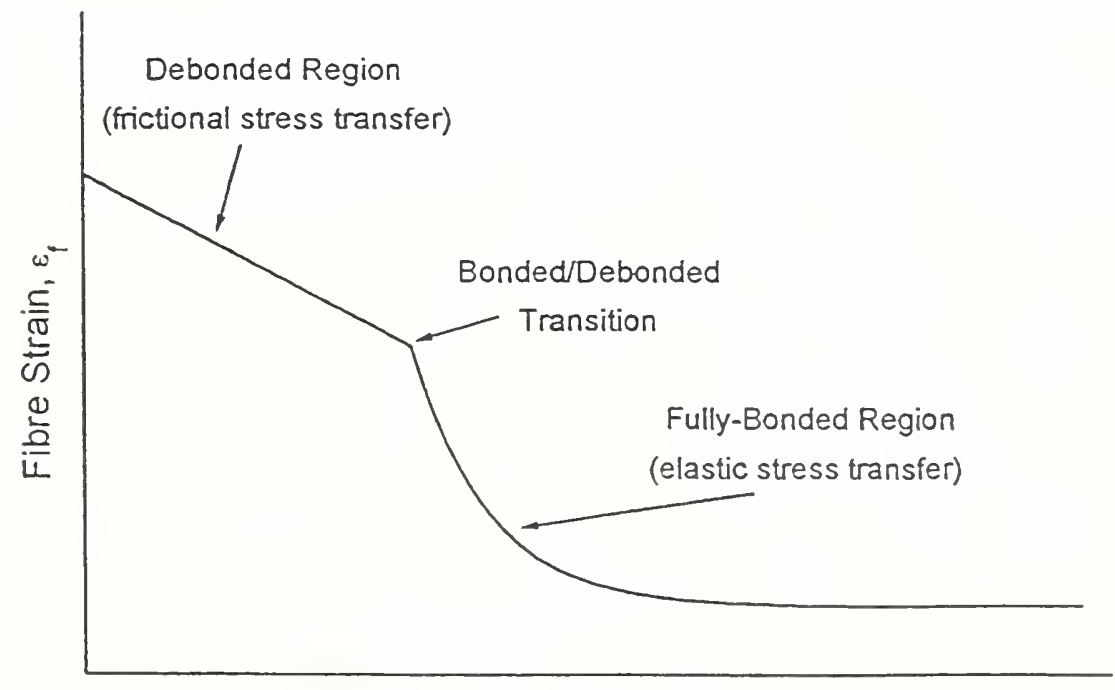

Distance along fibre, $x$

*Bannister D.J., Andrews M.C., Cervenka A.J., Young RJ., Comp. Sci. \& TechnoL 53 (1995) 411.

S1ide 39. 
Schematic diegram of the strain distnbutim in a cack-broguy frbe whenthe cack is closed.

\section{CRACK CLOSURE*}

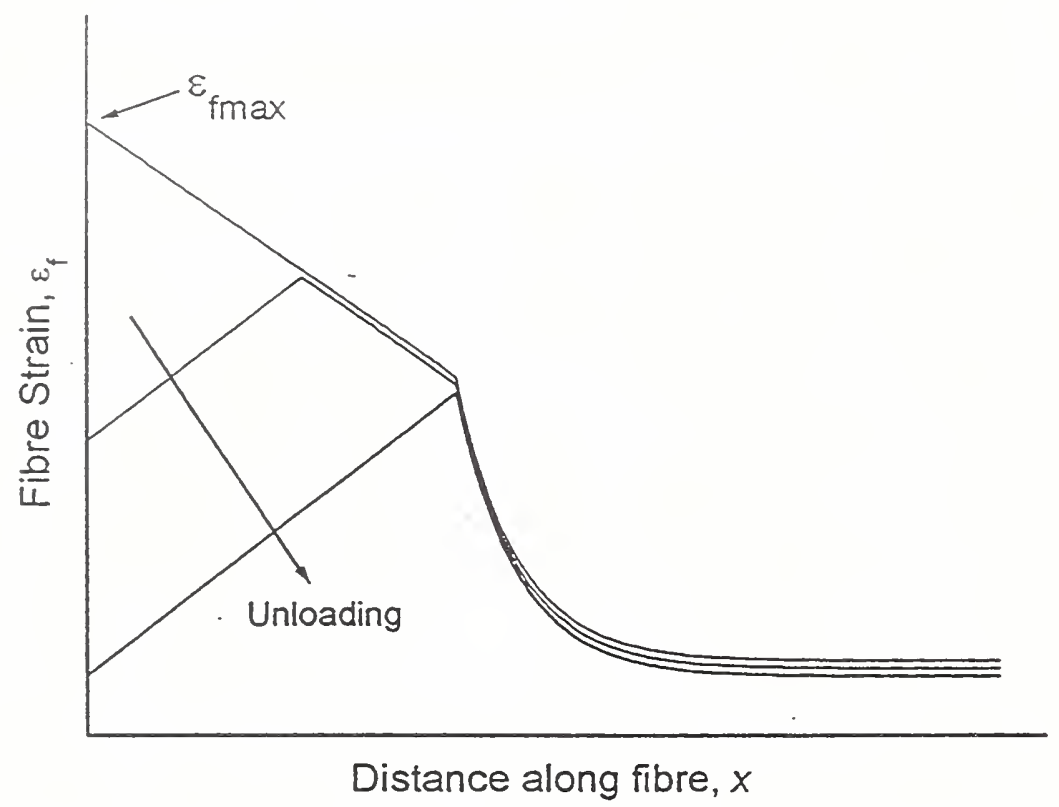

Schematic representation of fibre strain distribution during crack closure

\section{CRACK RE-OPENING*}

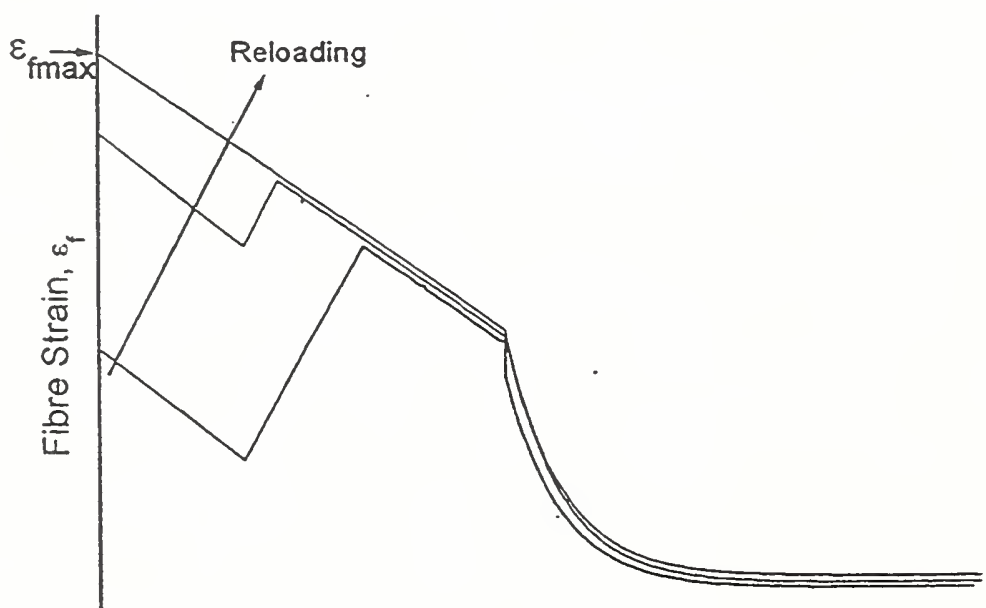

Distance along fibre, $x$

Schematic representation of axial fibre strain distribution during crack re-opening * Marshall D.B., Acta Metall. Mater. 40 (1992) 427. 


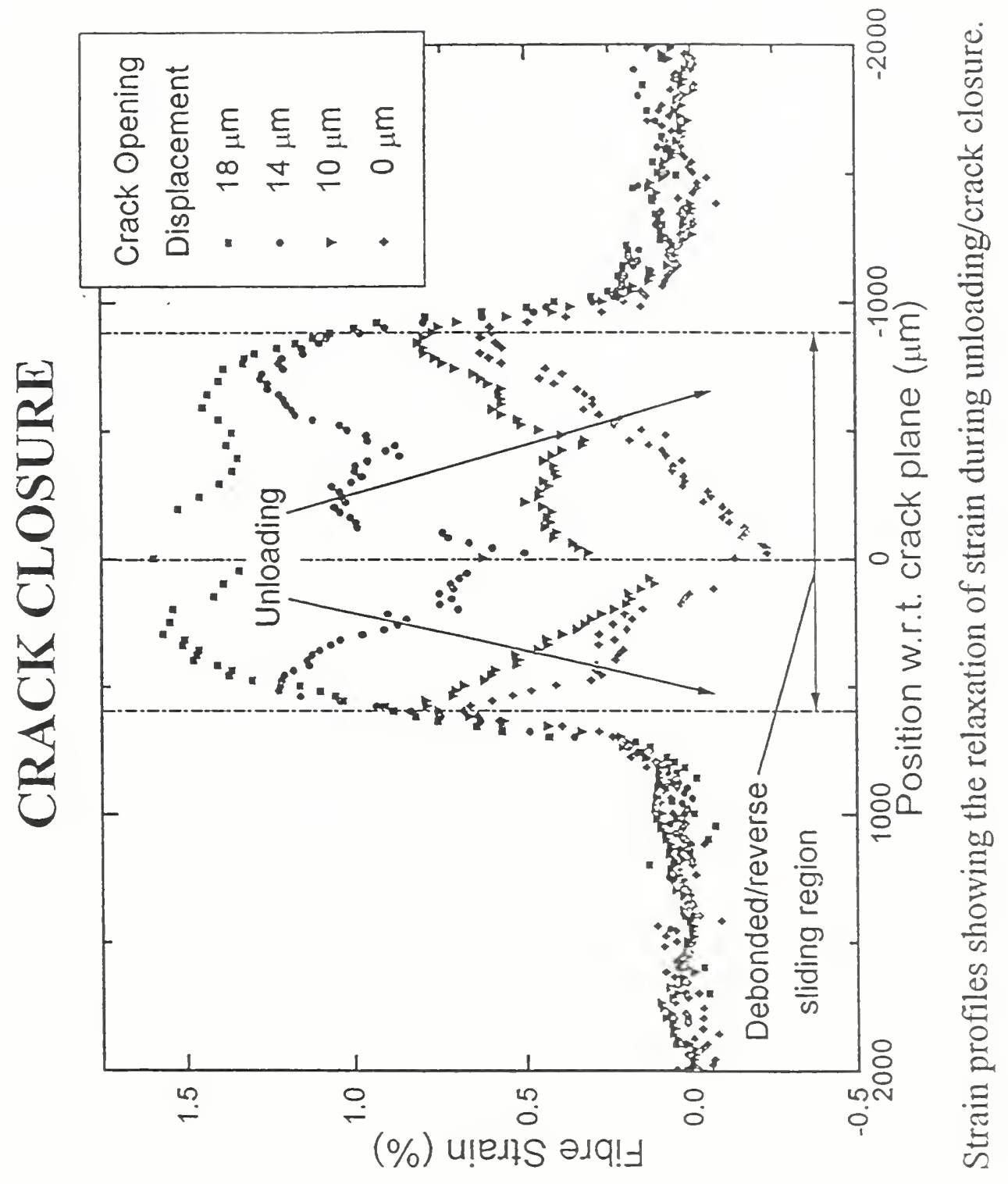

Expermental data shoury revese sheing durng cach closurefor an aramid/eproy specinen

Slide 41. 
Sclematis whestation of theoretical analysiof of

crack brogiry shmmy unvadiry and relvading

\section{CRACK BRIDGING - LOADING/UNLOADING}
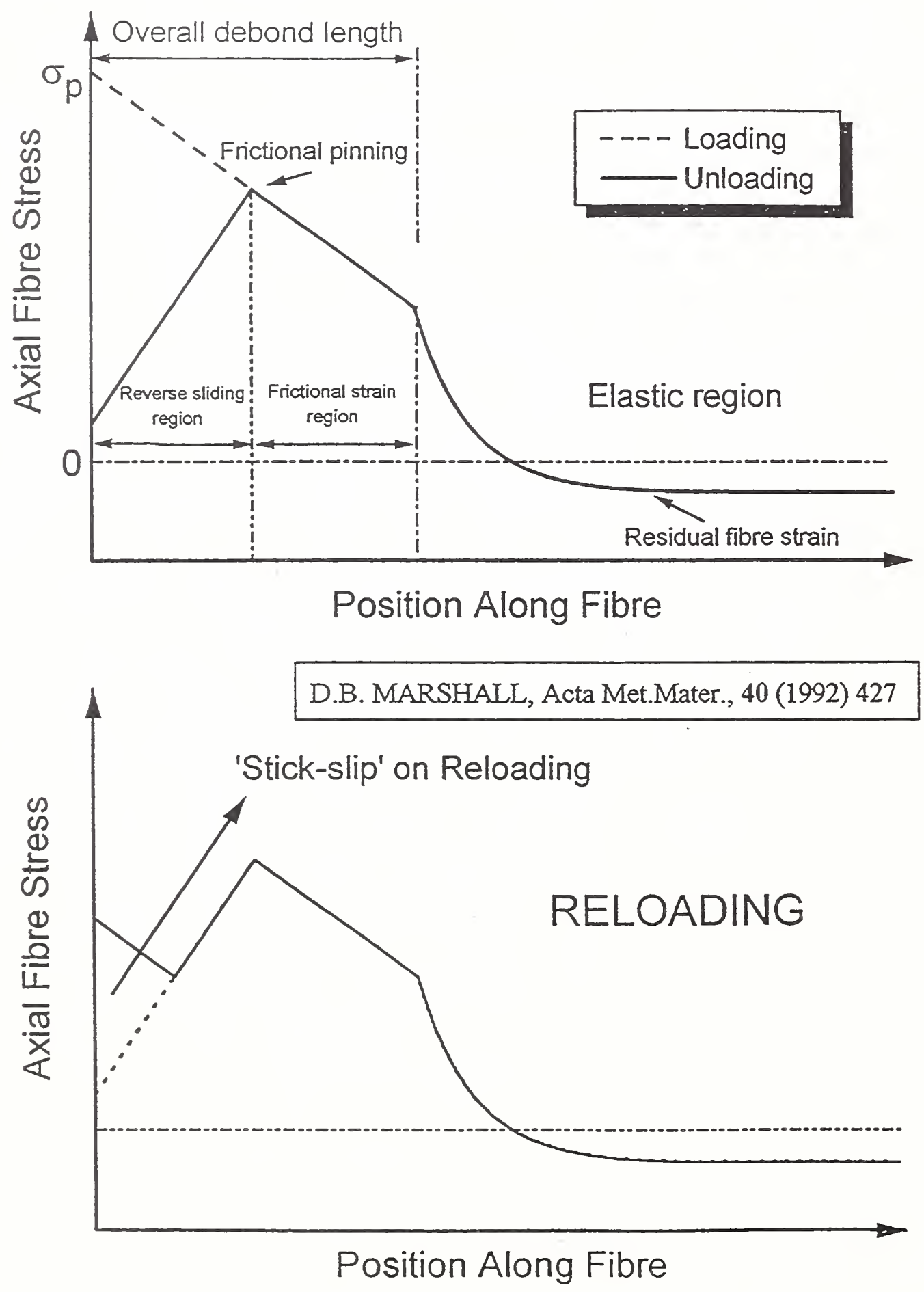

Slide 42. 


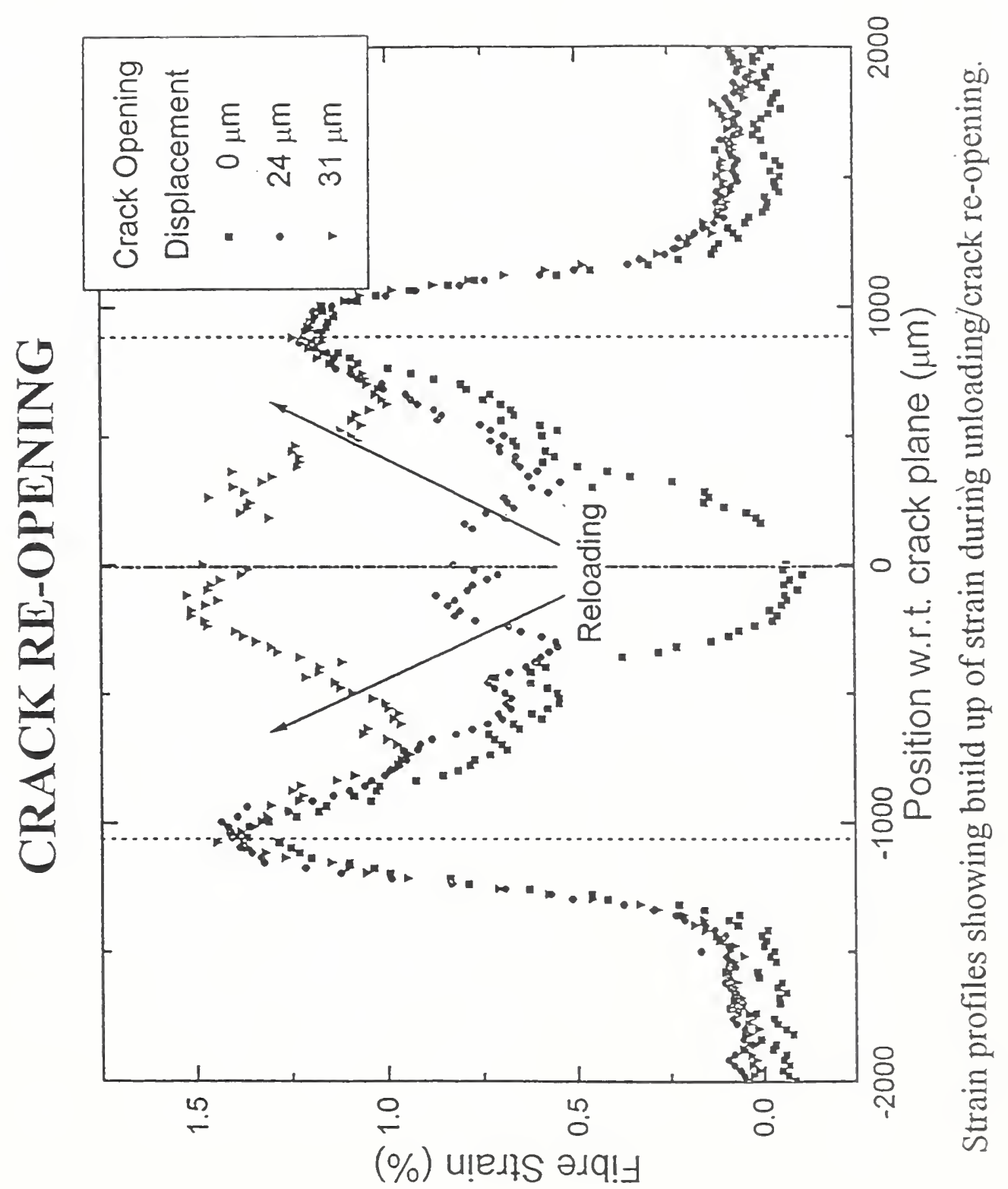

Expermental denmstraters of belainin on reloady frrative brolery a crack in an aranid/eposy

\section{Slide 43.}



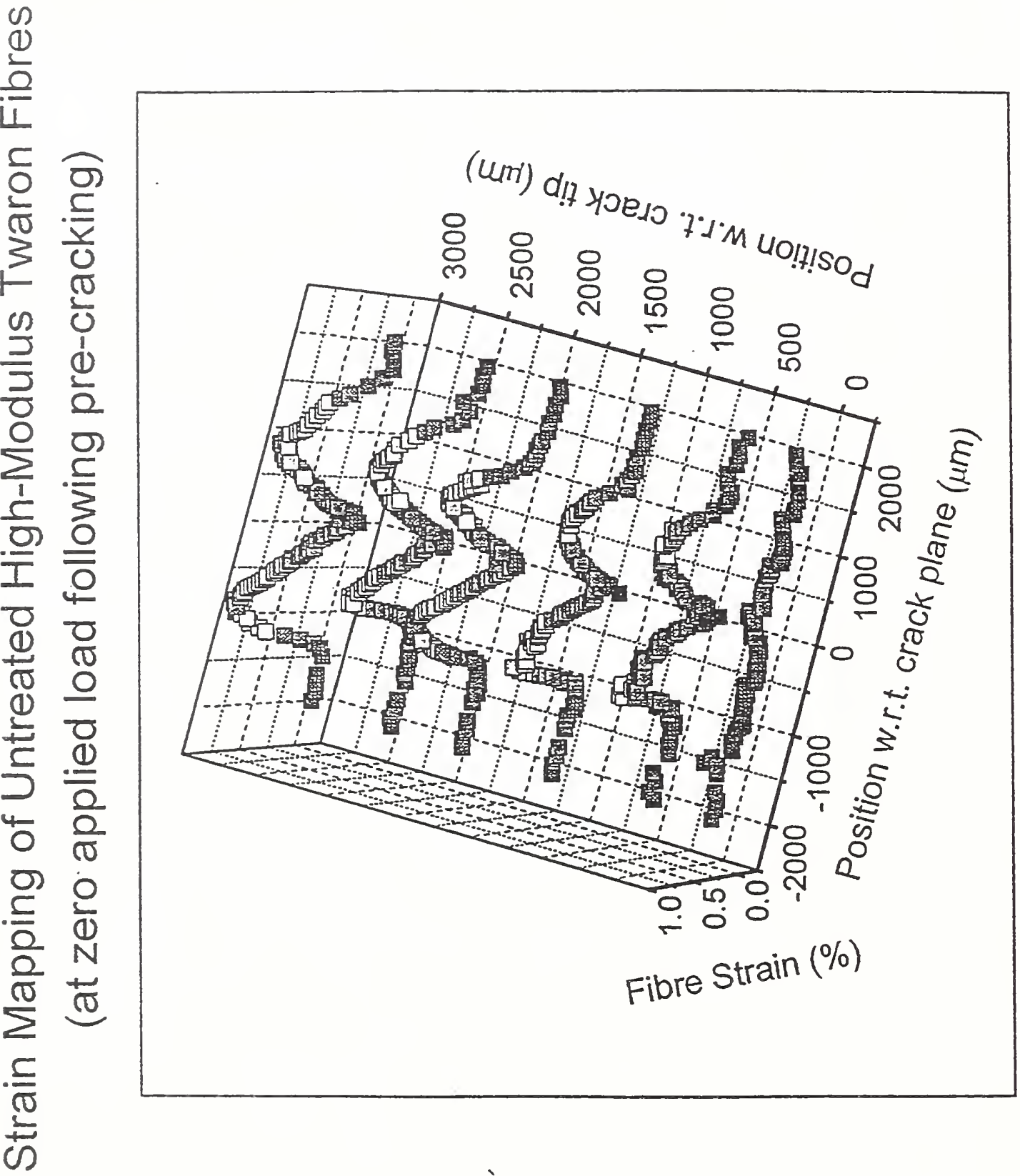

Expermental data shoming crack bredgry tor an aray of six hares. One hire is ahead of to crack tip and the otter fure are brolgry the crack.

Slide 44. 


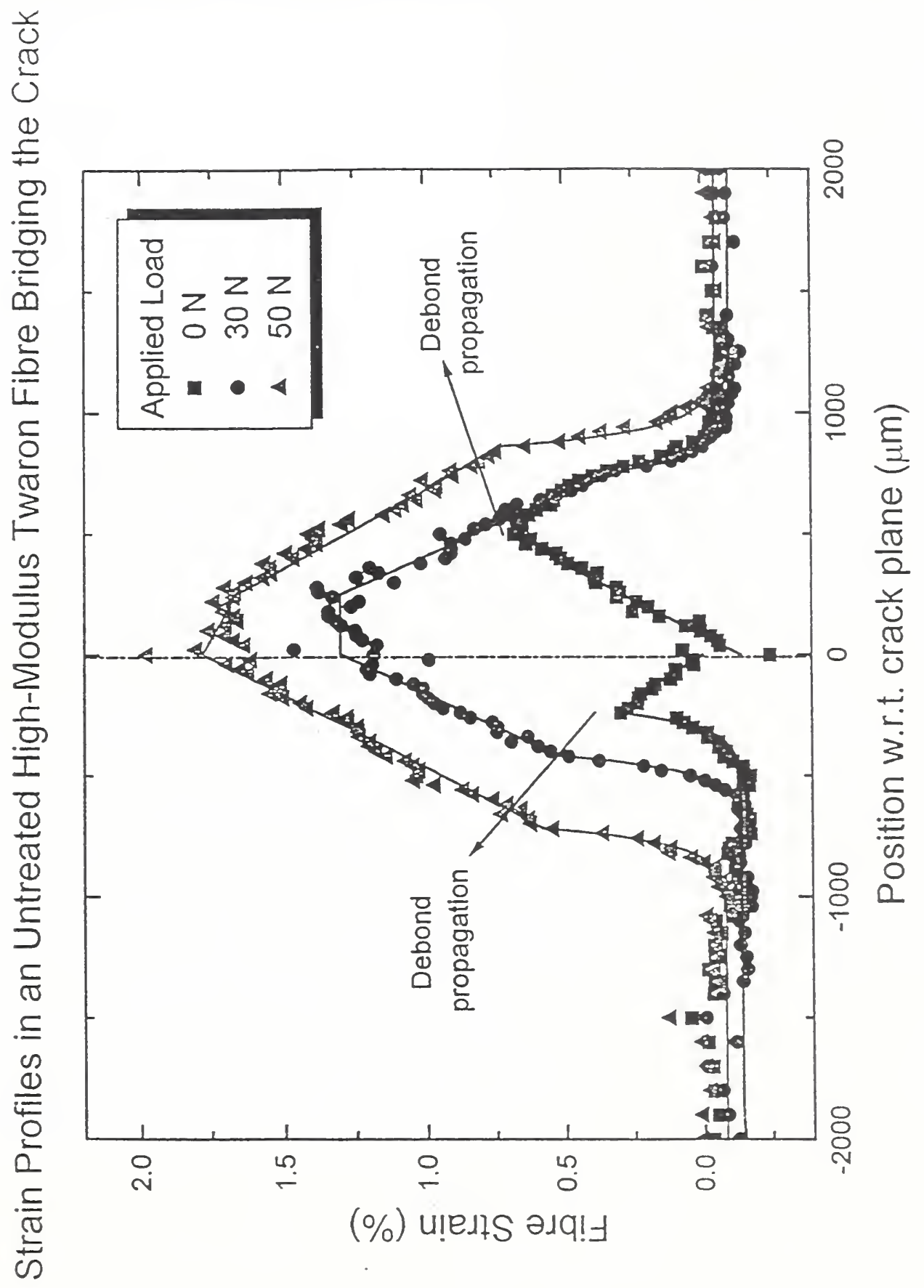

Detailed stomi prothes shony the propagation of

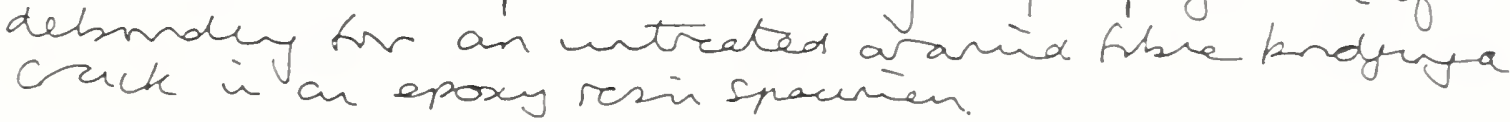

Slide 45. 


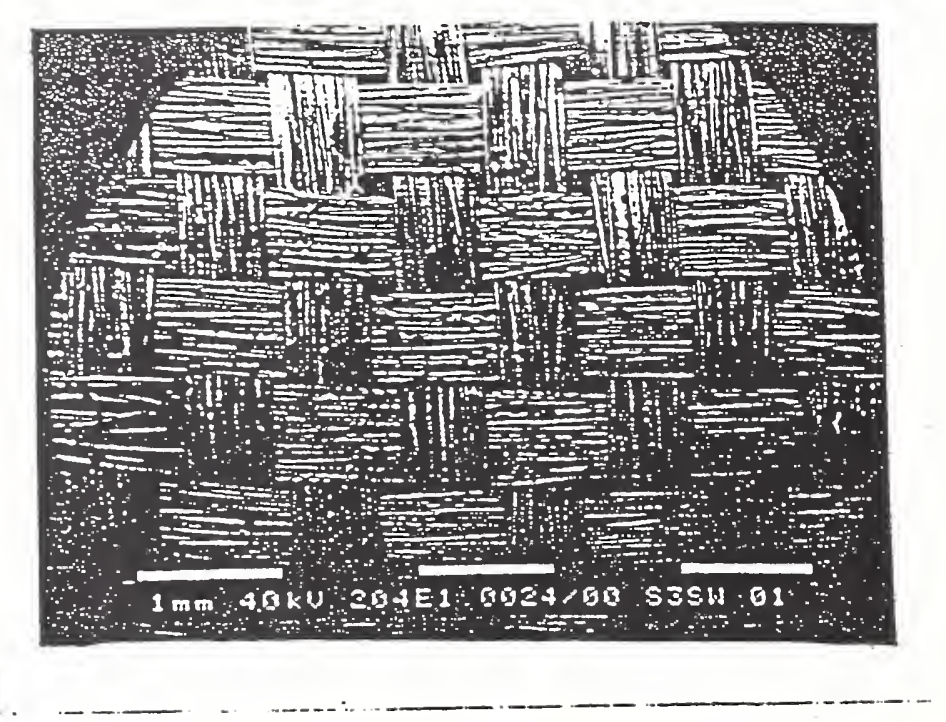

Figure 3.48 SEM micrograph taken under low magnification (-<20), showing the pla weate fabric (K285).

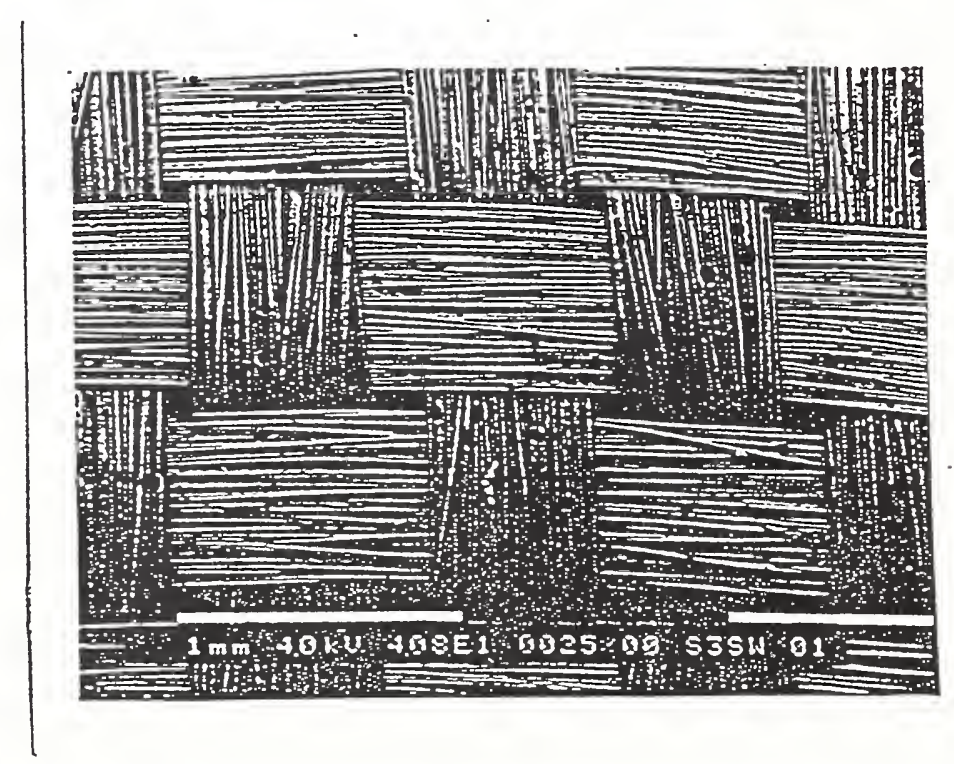

Figure 3.49 SEM micrograph taken under low magnification $(\times 40)$, showing the $\mathrm{pl}$ weave fabic (K120).

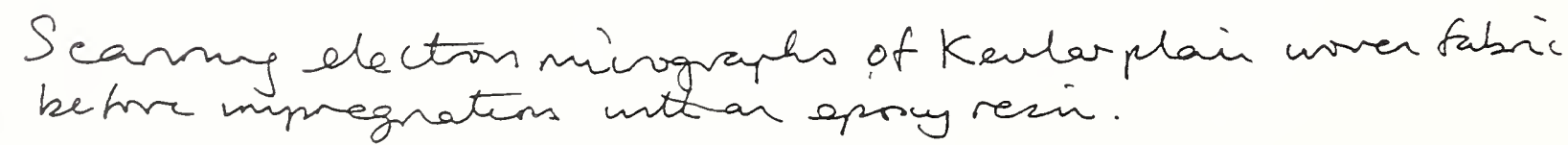

Slide 46. 


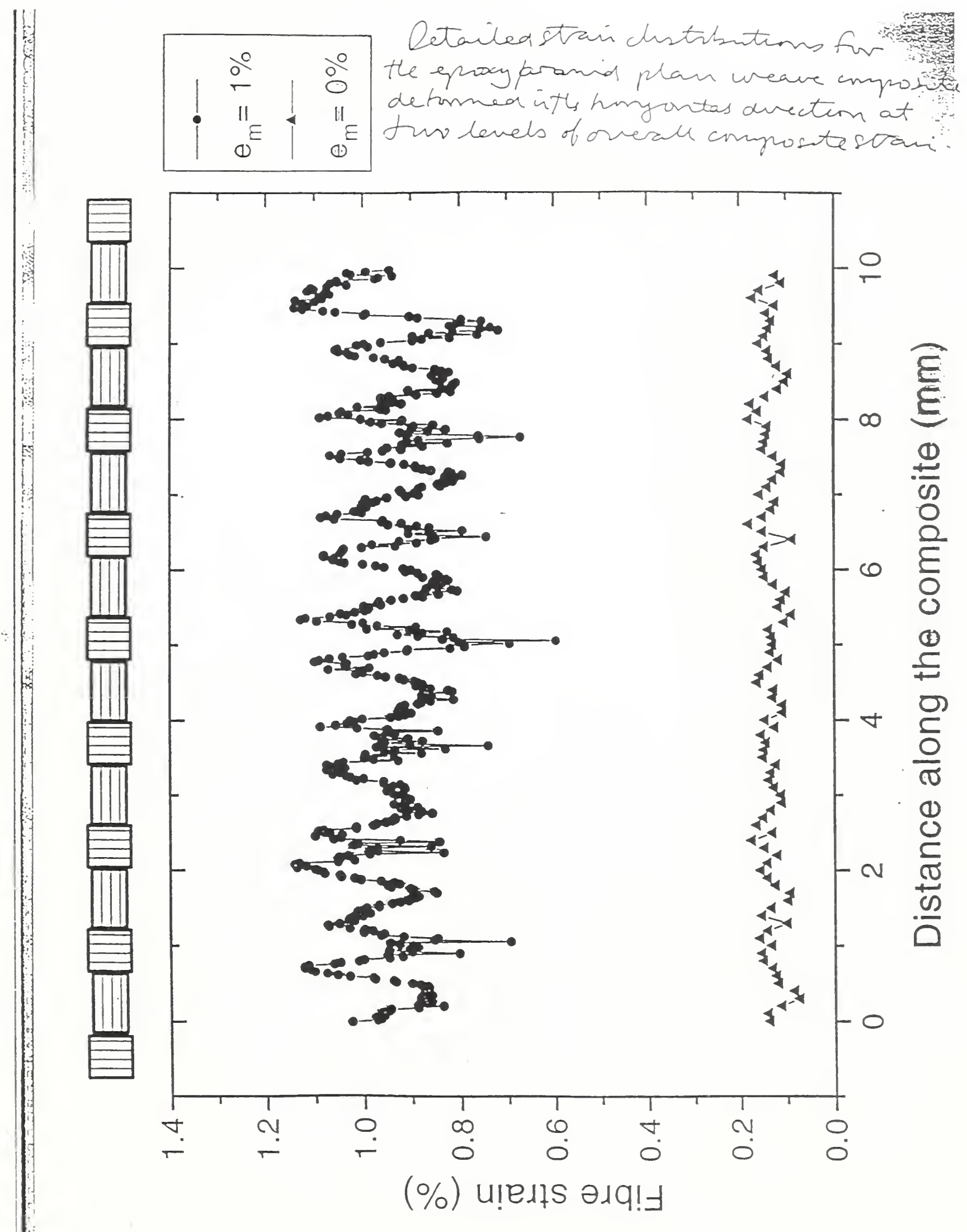

Slide 47. 


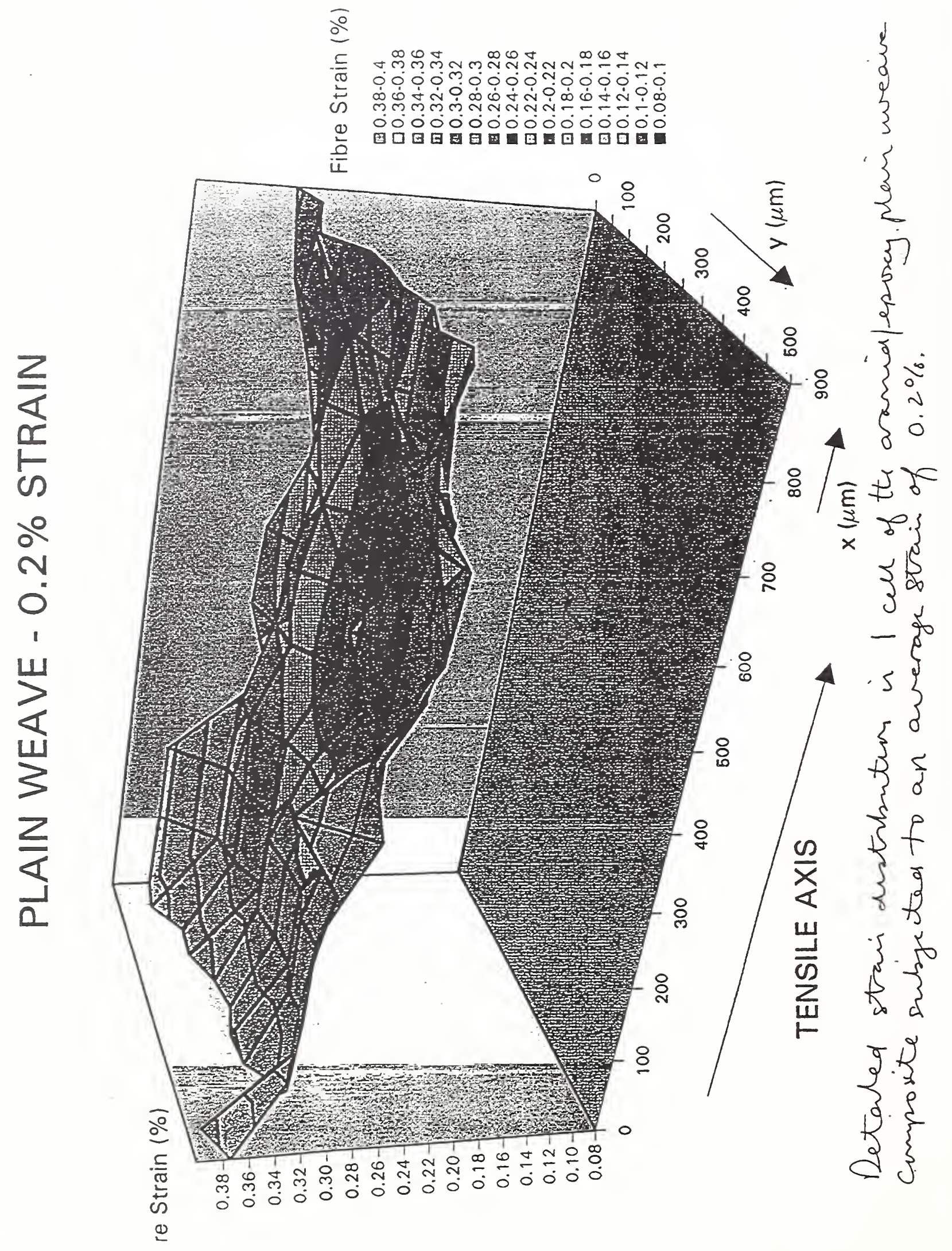

Slide 48. 


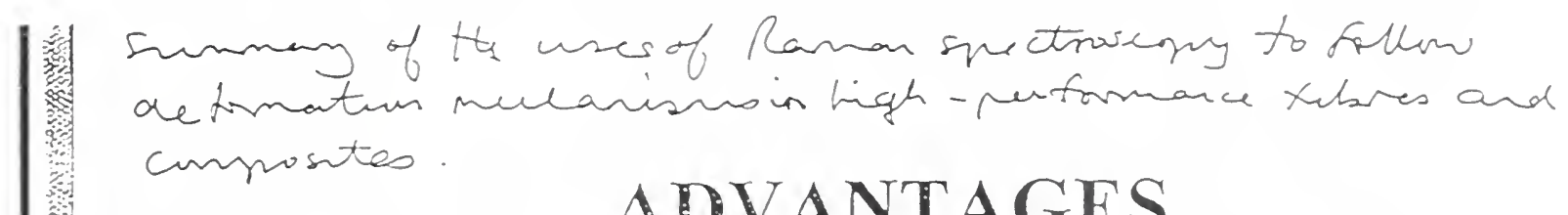

\section{ADVANTAGES}

- FIBRE DEFORMATION

Aramid, Rigid-Rod, Carbon, PE, PET, Ceramic Atomic/Molecular Deformation Processes

Tensile/Compressive Deformation

- MICROMECHANICAL TEST METHODS

In Situ Measurement of Fibre Stress

Most Test Methods can be Studied

Fibre/Matrix Adhesion

$\sim 2 \mu \mathrm{m}$ Resolution, $\pm 0.05 \%$ strain

- COMPLEX SYSTEMS

Local Fibre Deformation

Mapping/Remote-Sensing Possible

\section{LIMITATIONS}

- FIBRES

Glass Fibres Impossible

Some Carbon Fibres Difficult

- MATRICES

Opaque Matrices Difficult

Fluorescence gives Problems

- FUll COMPOSITES

Only Surface Fibres 


